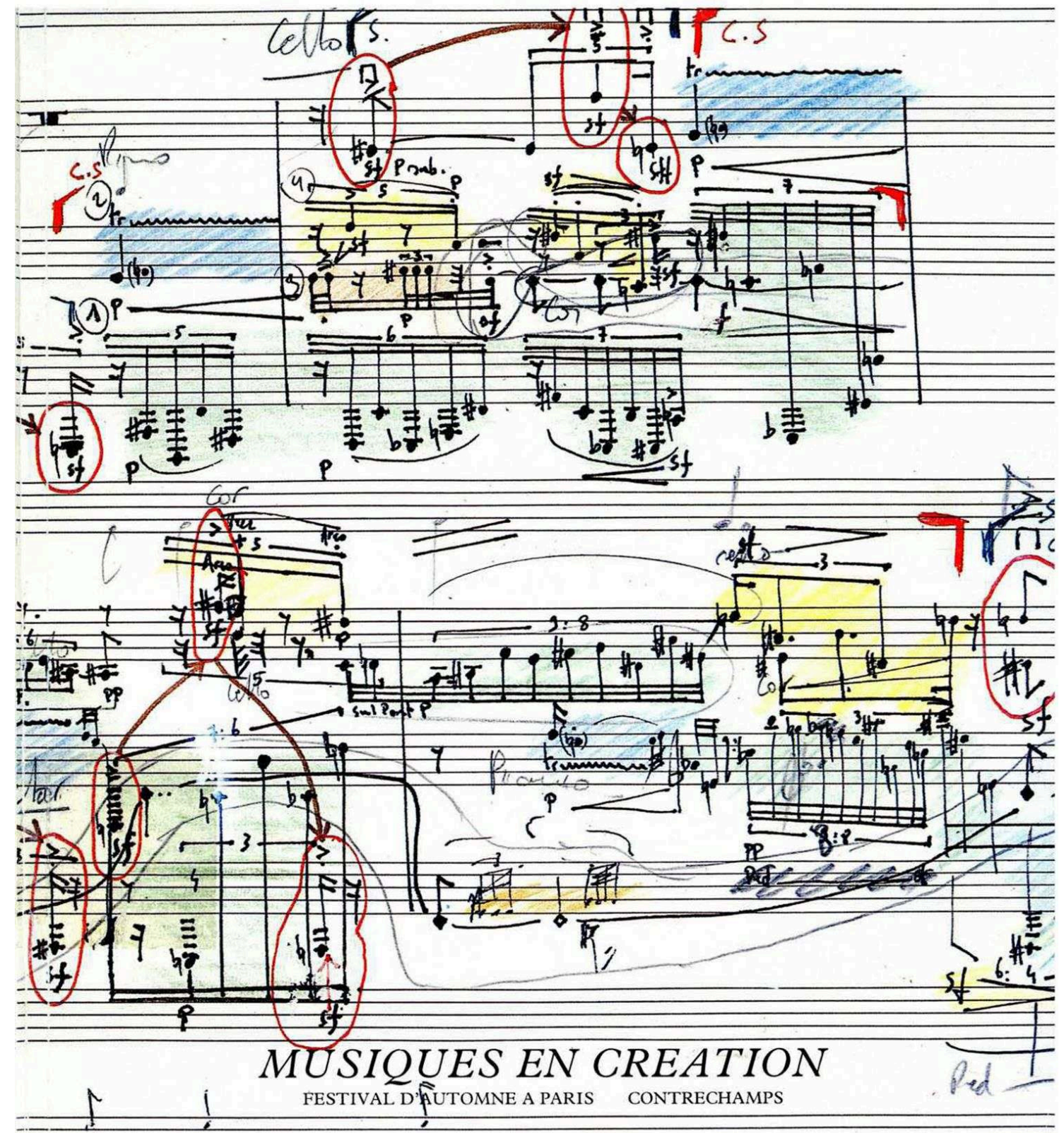




\section{Musiques en création}

Revue Contrechamps / numéro spécial

Philippe Albèra (dir.)

DOI : 10.4000/books.contrechamps.2251

Éditeur : Éditions Contrechamps

Année d'édition : 1989

Date de mise en ligne : 22 mars 2018

Collection : Essais sur les œuvres

ISBN électronique : 9782940599127

\section{Oponatition ebooks}

http://books.openedition.org

Édition imprimée

Nombre de pages : 208

Référence électronique

ALBÈRA, Philippe (dir.). Musiques en création : Revue Contrechamps / numéro spécial. Nouvelle édition [en ligne]. Genève : Éditions Contrechamps, 1989 (généré le 01 février 2021). Disponible sur Internet : <http://books.openedition.org/contrechamps/2251>. ISBN : 9782940599127. DOl : https://doi.org/ 10.4000/books.contrechamps.2251.

Ce document a été généré automatiquement le 1 février 2021. Il est issu d'une numérisation par reconnaissance optique de caractères.

(C) Éditions Contrechamps, 1989

Conditions d'utilisation:

http://www.openedition.org/6540 
SOMMAIRE

Avant-propos

Michel Guy

Médiations

Philippe Albèra

Questions aux compositeurs

\section{Réponses}

\section{Franck Krawczyk}

Le sérialisme

Le concept de musique pure

Fonction de la musique d'aujourd'hui

Technique et pensée musicale

Musique contemporaine et institutions

Musique d'aujourd'hui et tradition

L'œuvre

\section{Eric Tanguy}

Le compositeur

Le sérialisme

Le concept de musique pure

Fonction de la musique aujourd'hui

Technique et pensée musicale

Musique contemporaine et institutions

Musique d'aujourd'hui et traditions

L'œuvre

\section{Jacques Demierre}

\section{Marco Stroppa}

Le compositeur

Le sérialisme

Le concept de musique pure

Fonction de la musique aujourd'hui

Technique et pensée musicale

Musique d'aujourd'hui et traditions

\section{Philippe Manoury}

Le compositeur

Le sérialisme

Le concept de musique pure

Fonction de la musique aujourd'hui

Technique et pensée musicale

Musique contemporaine et institution

L'industrie

Le Ministère. Pourquoi y a-t-il autant de compositeurs dans ses murs?

Musique d'aujourd'hui et traditions

L'œuvre 


\section{Jean-Claude Eloy}

Le compositeur

Le sérialisme

Le concept de musique pure

Fonction de la musique aujourd'hui

Technique et pensée musicale

Musique contemporaine et institutions

Musique d'aujourd'hui et traditions

L'œuvre

\section{Anthony Braxton}

Le compositeur

Le sérialisme

Le concept de musique pure

Fonction de la musique

Technique et pensée musicale

Musique contemporaine et institutions

Musique d'aujourd'hui et traditions

L'œuvre

\section{Steve Reich}

Le compositeur

Le sérialisme

Le concept de musique pure

Fonction de la musique d'aujourd'hui

Technique et pensée musicale

Musique contemporaine et institutions

Musique d'aujourd'hui et traditions

L'œuvre

\section{Sylvano Bussotti}

Le compositeur

Le sérialisme

Le concept de musique pure

Fonction de la musique d'aujourd'hui

Technique et pensée musicale

Musique contemporaine et institution

Musique d'aujourd'hui et traditions

L'œuvre

\section{Toru Takemitsu}

Le compositeur

Le sérialisme

Le concept de musique pure

Fonction de la musique

Technique et pensée musicale

Musique contemporaine et institutions

Musique d'aujourd'hui et traditions

L'œuvre 


\section{Luciano Berio}

\section{Marcel Landowski}

Le compositeur

Le sérialisme

Le concept de musique pure

Fonction de la musique d'aujourd'hui

Technique et pensée musicale

Musique contemporaine et institutions

Musique d'aujourd'hui et traditions

L'œuvre

\section{Henri Dutilleux}

Les influences

Le sérialisme

Le concept de musique pure

Fonction de la musique d'aujourd'hui

Technique et pensée musicale

Musique contemporaine et institutions

Musique d'aujourd'hui et traditions

L'œuvre

Entretien avec Pierre Boulez

Philippe Albèra

Entretien avec Iannis Xenakis

Philippe Albèra

Entretien avec György Ligeti

Philippe Albèra

\section{Entretien avec Georges Aperghis}

Philippe Albèra

Quatre aspects du matériau musical et de l'écoute

Helmut Lachenmann

L'erreur comme nécessité

Luigi Nono

Le nom de la liberté

Mauricio Kagel

Entretien avec Peter Eötvös

Philippe Albèra

Entretien avec Diego Masson

Philippe Albèra

Entretien avec le Quatuor Arditti

Philippe Albèra

Stratégies picturales - le paradigme musical Albert Ayme 
Innovation et conséquences

Histoire des problèmes compositionnels

Giselher Schubert

Technique dodécaphonique et logique harmonique

Musique sérielle et musique aléatoire

Progrès et restauration

Nouvelle objectivité

Populisme

Absence d'orientation ou multiplicité

\section{La musique post-moderne}

Produit de la société de consommation

Susumu Shono

L'éclectisme comme poétique de la musique post-moderne

"Pseudomorphose" sur la logique de l'image

La musique du passé comme milieu et environnement

De la notion d'"expression" à celle d'“être témoin"

\section{Musique et référence}

Christian Corre

\section{Rejet de la pensée du matériau?}

Carl Dahlhaus

\section{Événements, Instruments, Monuments}

Francine Markovits

Rien de nouveau, dit le soleil

Les abeilles et le blues

La bibliothèque des instruments

Le clavecin des couleurs 


\section{NOTE DE L'ÉDITEUR}

La plupart des textes et entretiens publiés dans ce numéro spécial de la Revue Contrechamps / Festival d'Automne à Paris 1989 ont été réédités avec des modifications substantielles dans l'ouvrage Musiques en Création (Contrechamps, 1997). Ce sont les textes de cette dernière version qui doivent être utilisés comme référence. 


\title{
Avant-propos
}

\author{
Michel Guy
}

1 Lorsque j'ai réfléchi à ce que le Festival d'Automne devait faire en cette année du bicentenaire de la Révolution Française, je suis rapidement arrivé à la conclusion qu'il fallait opter pour un programme de commandes et de créations : non seulement parce que cela représente le fondement même de ce festival, qui a été conçu dans l'esprit de susciter et de promouvoir des œuvres et des démarches nouvelles, mais aussi parce que l'idée est, comme celle de révolution, une idée dérangeante.

2 Alors que le patrimoine constitue un facteur de rassemblement sur des valeurs communes, la création provoque des discussions, des dissensions, des questionnements, des prises de position; elle mobilise notre imagination et notre subjectivité, elle nous engage totalement. A travers elle, c'est l'abord des valeurs que la collectivité n'a pas encore reconnues, ou retenues, mais qui orientent l'évolution future. Depuis l'ouverture en 1977 du Centre Georges Pompidou, lieu de création, de confrontation des idées et des sensibilités, on a cessé de ricaner sur l'art contemporain. Le Festival d'Automne est né, en 1972, d'une même exigence, et il répond aux mêmes besoins. Il faut, en effet, que la société moderne se reconnaisse et s'interroge à travers les images sans cesse changeantes que la création lui tend.

3 Tel est le sens de la conception du programme musical 1989 du Festival d'Automne à Paris. 


\title{
Médiations
}

\author{
Philippe Albèra
}

1 En sollicitant des créations auprès d'une trentaine de compositeurs, le Festival d'Automne à Paris 1989 propose un panorama de la musique actuelle. En demandant à ces mêmes compositeurs de répondre à un questionnaire volontairement très général, et par là un peu provocateur, Contrechamps dresse le bilan de leurs idées et de leurs réflexions : certains ont préféré l'entretien à l'écriture, d'autres n'ont pu nous envoyer leurs réponses, faute de temps.

2 L'ensemble de ces œuvres et de ces matériaux donne une image de la situation présente, qui apparaît pour beaucoup comme "confuse" : la coexistence des tendances les plus diverses ne laisse pas apparaître une direction précise, la multiplication des enjeux les plus contradictoires rend impossible toute synthèse. Dans leurs déclarations, les compositeurs évitent les prises de position trop radicales, ainsi que toute polémique, et ils ne s'attardent guère sur des problématiques qui furent autrefois au centre des préoccupations. Il est difficile de dire si c'est là un signe de plus grande tolérance ou celui d'un repli sur soi, s'il faut y déceler une relative indifférence à des courants désormais trop nombreux ou trop éloignés les uns des autres, ou l'incapacité pure et simple à penser la situation présente dans toute sa complexité. Comment dessiner le visage de la création, entre ceux qui subordonnent la transformation de la pensée musicale au renouvellement du matériau - et pas uniquement dans le domaine électro-acoustique - et ceux qui investissent, sans la moindre naïveté, les valeurs expressives de moyens traditionnels comme l'orchestre symphonique, les formations standardisées de la musique de chambre, ou des genres musicaux tels que le concerto de soliste, la symphonie et l'opéra? Encore faudrait-il distinguer ici les compositeurs dont l'écriture absorbe ces caractéristiques traditionnelles de ceux dont l'invention y reste soumise. Comment s'orienter, toutefois, entre la tendance à une complexité croissante de l'écriture, qui prolonge une tradition centrale de la musique au $\mathrm{XX}^{\mathrm{e}}$ siècle, et celle qui, s'appuyant sur des expériences plus marginales, se veut plus sélective, voire minimaliste?

3 Ce qui, au cours de ce siècle, a formé des phases successives de l'histoire de la musique, ou tout au plus s'était opposé sur la base de traditions nationales divergentes, est aujourd'hui rassemblé dans un présent multiforme, contradictoire et déroutant. 
L'œuvre doit-elle accueillir, et organiser selon une combinatoire nouvelle, cette multiplicité de matériaux, de styles, de pensées, ce «métissage » typique d'une culture qui a su rendre simultanément disponibles toutes les musiques de notre histoire, en même temps que celles des aires géographiques, culturelles et sociologiques les plus éloignées? Certains voient resurgir ici le spectre des années vingt, avec les emprunts indifférenciés au répertoire traditionnel, au jazz et aux musiques exotiques, bref, une esthétique de l'hétérogène. Faut-il alors que l'œuvre transcende cette surabondance d'informations et d'influences, dans la rigueur d'une écriture débarrassée de toute référence trop explicite? L'invention aujourd'hui est-elle limitée à la réinterprétation de ce qui nous entoure, passé et présent confondus, ou doit-elle être une exploration de l'inouï, la préfiguration du futur? Quelques-uns des textes musicologiques de ce volume posent la question à travers une réflexion sur le concept de post-modernité (Shono), sur l'usage des références et des citations dans la musique récente (Corre), ou sur le développement de la pensée compositionnelle (Schubert) et la dialectique entre expression et matériau (Dahlhaus).

4 Ces questions ne sont à vrai dire guère nouvelles. Elles sont apparues plus d'une fois au cours de ce siècle, et ont leurs origines dans la musique du XIX ${ }^{e}$ siècle. Elles ne proviennent pas seulement de l'individualisation croissante de l'acte créateur et des conditions sociales dans lesquelles la musique dite "sérieuse » évolue, mais aussi de l'impossibilité pour la musique moderne d'être reliée à une totalité signifiante (croyance, pensée philosophique ou politique, mythologie, monde affectif, etc.) qu'elle pourrait réfracter dans son langage propre. Ce qui a déjà été observé dans le domaine philosophique par exemple, touche également le domaine musical, et sape ses anciens fondements. Le désir de retrouver cette unité perdue, cette plénitude, apparaît déjà chez Wagner sous la forme d'une image théâtrale, d'une allégorie. On le retrouve plus tard dans la célébration de la nature, dans un certain "primitivisme", dans l'influence des idiomes populaires ou des musiques extra-européennes, dans le retour à la tradition, dans différentes formes de mysticisme, ou encore dans l'élaboration de systèmes musicaux rigoureux, clos sur eux-mêmes, garants d'un absolu débarrassé de toute contradiction. Mais on le retrouve aussi dans l'expression de son impossibilité même, à travers l'image de l'homme devenu étranger à lui-même comme à sa société et à sa culture. En un ultime geste de salut, la totalité de l'être est alors mobilisée dans l'immédiateté expressive du tragique, dans le montage d'éléments fragmentaires, souvent traités sur un mode critique ou ironique, dans la superposition aléatoire des bribes de notre présent.

5 Aujourd'hui encore, toutes ces attitudes coexistent, sans que plus personne n'ose évoquer l'idée d'un "style commun" - visé par le mouvement sériel dans les années vingt et dans les années cinquante - ou définir avec assurance une direction particulière, comme ce fut le cas dans les années soixante. On se méfie, plus que jamais, de la réflexion théorique, souvent jugée inutile ou trompeuse; les différentes démarches ne sont plus guère définies, ni par rapport au présent, ni par rapport au passé : le temps des déclarations d'intentions est révolu. Les textes et les entretiens publiés ci-après en témoignent dans une certaine mesure: la composition y est présentée le plus souvent comme un "mystère", et la fonction sociale de la musique d'aujourd'hui, à l'heure de la communication triomphante, laisse la plupart des compositeurs perplexes. La création semble devoir s'épuiser dans son propre artisanat. D'où l'idée que le meilleur critère d'appréciation pour une œuvre nouvelle serait finalement celui du «métier» qu'elle révèle ou non chez le compositeur; mais 
l'exemple de certaines démarches utopiques au cours de ce siècle a rendu l'argument contestable. Il l'était déjà chez les novateurs du siècle dernier: sans parler des "maladresses" d'orchestration chez Schumann ou des "aberrations" harmoniques de Berlioz, il faut rappeler que les dernières œuvres de Beethoven furent considérées par des compositeurs et des interprètes sérieux comme mal écrites du point de vue instrumental, voire injouables (on invoquait alors sa surdité...). En appeler au concept d'authenticité, qui fut revendiqué plus d'une fois au cours du vingtième siècle pour dépasser les critères académiques, n'est guère plus aisé : il représente une forme de la subjectivité critique cherchant à saisir un objet de la subjectivité créatrice. Sa justification est périlleuse vis-à-vis de ceux qui en contestent le bien-fondé chez tel ou tel compositeur.

6 L'absence de critères objectifs à partir desquels aborder la création contemporaine, qui est évidemment liée à l'absence de tout critère objectif dans la composition, rend toute tâche critique particulièrement problématique. Toutefois, il semble un peu facile de s'en tirer en disant que "ce dont on ne peut parler, il faut le taire " (Wittgenstein). Même si le processus de création demeure mystérieux, si la logique de l'œuvre se dérobe, au bout du compte, à toute analyse, la réflexion peut appréhender ce que l'œuvre met en jeu, aussi bien dans sa spécificité (matériaux, techniques, pensée, etc.) que dans les significations et les émotions qu'elle engendre. Il ne s'agit pas, évidemment, d'entériner l'idée simpliste selon laquelle un discours critique peut venir à bout d'une œuvre ou d'une démarche-on sait à quel point le langage musical est, dans son essence, irréductible au langage verbal - mais d'insister sur la nécessité de la connaissance et d'un travail inlassable de déchiffrement, qui sont seuls capables de nous mener au-delà des apparences, des conventions, du savoir, soit à une authentique liberté. Le refus de toute médiation par la pensée, qui est devenu courant dans les dernières années (y compris chez des compositeurs qui avaient adopté une attitude différente dans le passé), est moins le signe d'une libération face à ce qui aurait autrefois entravé la libre imagination musicale - un abus de la "théorie" - que le symptôme d'une soumission aux critères les plus traditionnels et les plus académiques, si ce n'est aux impératifs du marché de la communication. Nous sommes d'accord avec Helmut Lachenmann pour dire que «l'écoute sans le secours de la pensée est désarmée » (voir son texte ci-après). Ce sont d'ailleurs souvent les démarches les plus "spiritualistes" qui, au cours de ce siècle, ont provoqué le besoin le plus aigu de commentaires et d'éclaircissements, tandis que la philosophie de mouvements plus pragmatiques, comme le néo-classicisme de l'entre-deux-guerres, se résumait par la phrase trop célèbre de Stravinsky sur l'incapacité de la musique "à exprimer quoi que ce soit".

7 Or, il est toujours possible, et même souhaitable, d'analyser les relations qui existent entre l'intention, ou l'idée d'une œuvre, et sa réalisation, et de poser la question de l'articulation entre significations sous-jacentes et phénomènes perçus. Bien des œuvres actuelles, qui paraissent aux avant-postes de la pensée la plus novatrice, illustrent simplement leurs propres procédures et leurs propres matériaux, sortes d'autojustifications et d'autoglorifications un peu vaines. La série autrefois, la technologie aujourd'hui, ne sont pas en elles-mêmes des éléments de signification. En tant que moyens, elles réclament encore et toujours d'être utilisées comme nécessités expressives. Fétichisées, elles ne font que révéler un manque que la réflexion critique a justement pour tâche de dévoiler. Par ailleurs, bien des discours sur les moyens technologiques, comme jadis certains discours sur le sérialisme, s'empêtrent dans un 
concept progressiste et linéaire de l'histoire que Nietzsche avait critiqué il y a plus d'un siècle déjà : l'épuisement de certains moyens entraînerait ainsi la nécessité de moyens nouveaux, comme pour l'économie capitaliste, la saturation de certains marchés impose d'en découvrir d'autres. On ne peut croire aujourd'hui encore à un tel déterminisme historique, comme il est difficile d'accepter l'idée, rendue caduque par l'histoire elle-même, qu'un matériau musical donné devienne une fois pour toutes obsolète. Ceux qui, par le passé, ont défendu de telles idées, notamment quant à la subdivision de l'échelle sonore, ont administré la preuve malgré eux que l'histoire ne procédait pas par déductions logiques. Ce qui a pu paraître, à un moment donné, comme une évolution inéluctable, laissant de côté ce qui n'entrait pas dans son schéma, est vu aujourd'hui avec nuance, comme c'est le cas dans les textes de Schubert et de Dahlhaus ci-après; et Ligeti a raison de faire remarquer qu'une certaine conception de la modernité, notamment à l'époque de Darmstadt, avait refoulé des tendances tout à fait valables qui resurgissent aujourd'hui avec force.

8 Le plus troublant, dans la situation actuelle, n'est sans doute pas que les compositeurs aient renoncé à un travail "théorique" semblable à celui des années cinquante - qui correspondait alors à des motivations autres que simplement théoriques - mais qu'il soit si difficile à la plupart des compositeurs de déterminer, ne serait-ce qu'à titre individuel, la fonction de la musique - de sa propre musique - et ce précisément par rapport aux techniques et aux formes qu'elle met en jeu. Si l'œuvre contemporaine demande à être déchiffrée, ce n'est certes pas en fonction des critères déjà répertoriés dans quelque musique du passé, dont on croit souvent à tort avoir percé la signification. Et l'inventaire des moyens qu'elle met en œuvre, s'il apparaît jusqu'à un certain point nécessaire, ne dit pas le fin mot de l'énigme. Le jugement esthétique luimême, qui s'est développé à partir d'une appréhension historicisante de la musique, reste enfermé dans des catégories que l'œuvre nouvelle a justement pour mission de traverser. Son discours, finalement, est impuissant à rendre compte du processus par lequel l'œuvre nous entraîne vers des significations ou des émotions n'ayant pas encore été rationnalisées et qui par elles sont enfin dévoilées. Toute œuvre nouvelle, en effet, s'articule de façon particulière au réel : celui du monde dans lequel elle apparaît (et qui comprend aussi, bien évidemment, le monde des représentations intérieures), et celui des moyens, du langage de son époque. C'est pourquoi les critères esthétiques ou techniques fixés une fois pour toutes ne peuvent en saisir la spécificité. C'est aussi pourquoi la médiation du commentaire, tout d'abord celle des compositeurs euxmêmes, est nécessaire : elle aide à repérer et à approfondir le lien spécifique entre ces deux aspects du réel. Elle permet d'identifier ce qui constituait, dans le passé, une évidence, mais qui est aujourd'hui devenu problématique: le sens même de l'œuvre musicale.

9 La crise, que beaucoup de commentateurs perçoivent dans la musique d'aujourd'hui, au-delà d'un éclatement stylistique évident, n'est-elle pas davantage due à l'indétermination de son sujet, qu'à des question de langage et de matériau? Ne seraitil pas urgent que la discussion s'oriente un peu plus dans cette direction dans l'avenir le plus proche? N'est-ce pas à cette condition que le public pourra comprendre la nécessité de telle ou telle forme nouvelle, et en saisir véritablement l'enjeu? 


\section{Questions aux compositeurs}

7 En êtes-vous partisan? Dans quelle mesure la musique doit-elle s'allier au texte, au théâtre, à la danse, ou à d'autres formes d'expression?

8 contemporains - écrivains, plasticiens, hommes de théâtre, cinéastes, etc. - alimentent votre réflexion artistique?

9 Fonction de la musique aujourd'hui.

10 Quelle est la fonction de la musique aujourd'hui, et quel devrait être son rôle dans la société ?

11 Technique et pensée musicales

12 La musique contemporaine est-elle, dans certains cas, en rupture avec les principes dits naturels de la résonance et de la perception?

13 Comment envisagez-vous l'évolution des systèmes musicaux (nouvelles échelles, nouveaux matériaux, nouvelles formes de communication, etc.) ? L'ordinateur, ou les moyens électroacoustiques en général, vous semblent-ils indispensables pour la composition d'aujourd'hui? Imposent-ils une mutation radicale de la pensée et de la pratique musicales? 
15 Comment voyez-vous les liens entre la musique contemporaine et les institutions musicales traditionnelles - opéras, orchestres symphoniques, conservatoires? La vie musicale devrait-elle être réorganisée différemment?

Musique d'aujourd'hui et traditions

17 Comment envisagez-vous le rapport de la musique d'aujourd'hui avec la tradition, y compris celle des civilisations autres qu'européennes? Quel est votre rapport avec les idiomes populaires, traditions anciennes ou actuelles (comme le jazz ou le rock)?

18 L'œuvre.

19 De quelle manière naît une œuvre? Quel est le processus suivi, depuis l'idée initiale jusqu'à la réalisation finale? 
Réponses 


\section{Franck Krawczyk}

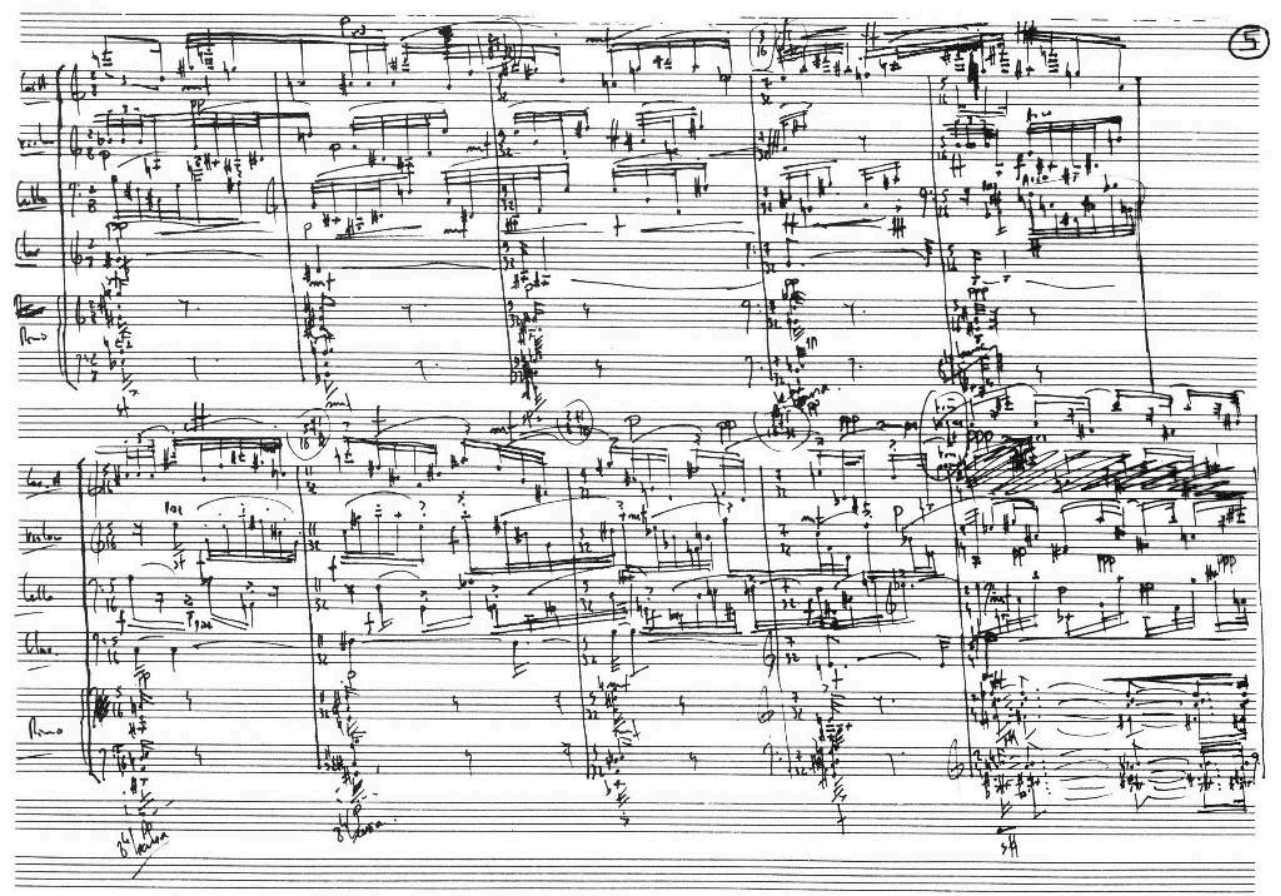

1 Le compositeur (Comment définissez-vous votre développement depuis vos débuts?)

2 Posez cette question à des compositeurs "plus développés" que moi !

3 (Votre évolution a-t-elle été déterminée par des influences, des découvertes, des circonstances extérieures importantes?)

4 - Oui. (Lesquelles?)

5 Les lectures de trois compositeurs : Schumann, Debussy, et Louis Couperin.

6 A mon sens, ces trois grands créateurs sont les maîtres incontestables du point, de la virgule, du mot, de la phrase. Ils ont compris l'importance de l'exclamation, de l'interrogation, de la suspension dans le discours musical. Ce sont les derniers prosodistes de l'histoire de la musique. Ils sont les "maîtres du silence".

7 (Quelle direction envisagez-vous pour l'avenir ?) 
8 - Ecrire. Quant à “pour l'avenir”, tant que je serai vivant, la question n'aura pas lieu d'être.

\section{Le sérialisme}

9 Le sérialisme n'est qu'un concept et rien d'autre. Il repose sur des bases aussi indéfendables que celles de l'harmonie, et comme tout concept, il ne possède pas d'esthétique propre.

10 Ce sont les compositeurs qui créent à partir d'un concept une esthétique. Dubois n'est pas plus créateur que Leibowitz. Nos oreilles s'habitueront un jour aux mauvaises compositions sérielles comme elles sont habituées aujourd'hui à certaines oeuvres classiques si insipides qu'on ne leur trouve plus pour défense que le simple fait qu'elles soient écrites dans un langage harmonique.

11 Je ne pense pas qu'il faille codifier systématiquement un langage afin qu'il devienne universel. Je crois davantage en l'individu, en sa capacité expressive, en son sens du théâtre, en son génie. Si une note contient tous ces messages, alors elle n'a pas à se préoccuper de la valeur du langage dans lequel elle est écrite et encore moins de sa codification. Elle sera comprise, voilà tout.

\section{Le concept de musique pure}

Une des erreurs sur laquelle nous vivons aujourd'hui repose sur une totale incompréhension de cette corrélation entre la musique et les autres arts. La musique peut tout à fait se passer au stade de la réalisation finale d'une chorégraphie, d'un texte ou d'un jeu de scène, mais parce qu'en elle, elle est justement un geste, un mot ou bien encore un masque. Elle est son propre théâtre.

(Quels domaines artistiques, autres que la musique, vous intéressent ?)

14 Tous.

15 (Quels artistes contemporains - écrivains, plasticiens, hommes de théâtre, cinéastes, etc. alimentent votre réflexion artistique ?)

16 J'ai beaucoup d'admiration aujourd'hui pour Claude Helffer. Pour reprendre une anecdote connue : "à défaut d'être le plus grand, il est le seul".

17 Quelques partitions contemporaines m'ont fortement impressionné pour de multiples raisons, techniques ou philosophiques, mais j'aurais du mal à dégager un nom, car cela me semblerait aussitôt incomplet.

\section{Fonction de la musique d'aujourd'hui}

18 La musique n'a pas de fonction prédéterminée dans la société, et les gens n'ont pas à recevoir de leçons musicales par des "pseudo-musiciens", qui feraient mieux d'en prendre ayant de parler. En revanche, il faut donner tous les moyens à la société de s'imprégner d'art. Pour cela, Gould a ouvert une voie qu'il ne faut pas négliger. C'est par l'image que la communication s'établira entre le compositeur et le public. 


\section{Technique et pensée musicale}

19

\section{Musique d'aujourd'hui et tradition}

23 aujourd'hui. Quant au rock ou d'autres formes de musique populaire, je dois observer
qu'il parvient à toucher beaucoup plus le public que toute autre forme de musique. Ce
qui veut dire que la musique contemporaine échoue indiscutablement dans sa fonction
communicative. J'aime le rock et je ressens le besoin d'écouter ce que les rockers ont à
nous dire, car je me rends compte alors davantage des désirs du public d'aujourd'hui. A
nous de ne pas tomber à partir de là dans le piège qui nous est tendu.

\section{L'œuvre}

Tant qu'une œuvre ne s'impose pas à moi dans sa totalité, je ne l'écris pas. Elle n'a pas le choix, elle est tout ou rien. J'écris très vite, mais l'oeuvre est déjà composée lorsque je me mets à la table ; il ne s'agit plus alors que de la recopier.

Sa naissance coïncide presque toujours avec sa mort. Il faut tuer une oeuvre pour en écrire une autre, c'est ce cyle qui entretient l'équilibre du créateur. Je peux ainsi vivre, manger, dormir en composant. Si je pense à Rimbaud, mon œuvre est un "vertige" que je tente de fixer.

Il faut tout donner à la création, jusqu'à la dernière goutte de son sang, sans se poser la moindre question ; si ce n'est pas le cas, alors la musique n'est rien qu'une carte postale sonore. 


\section{Eric Tanguy}

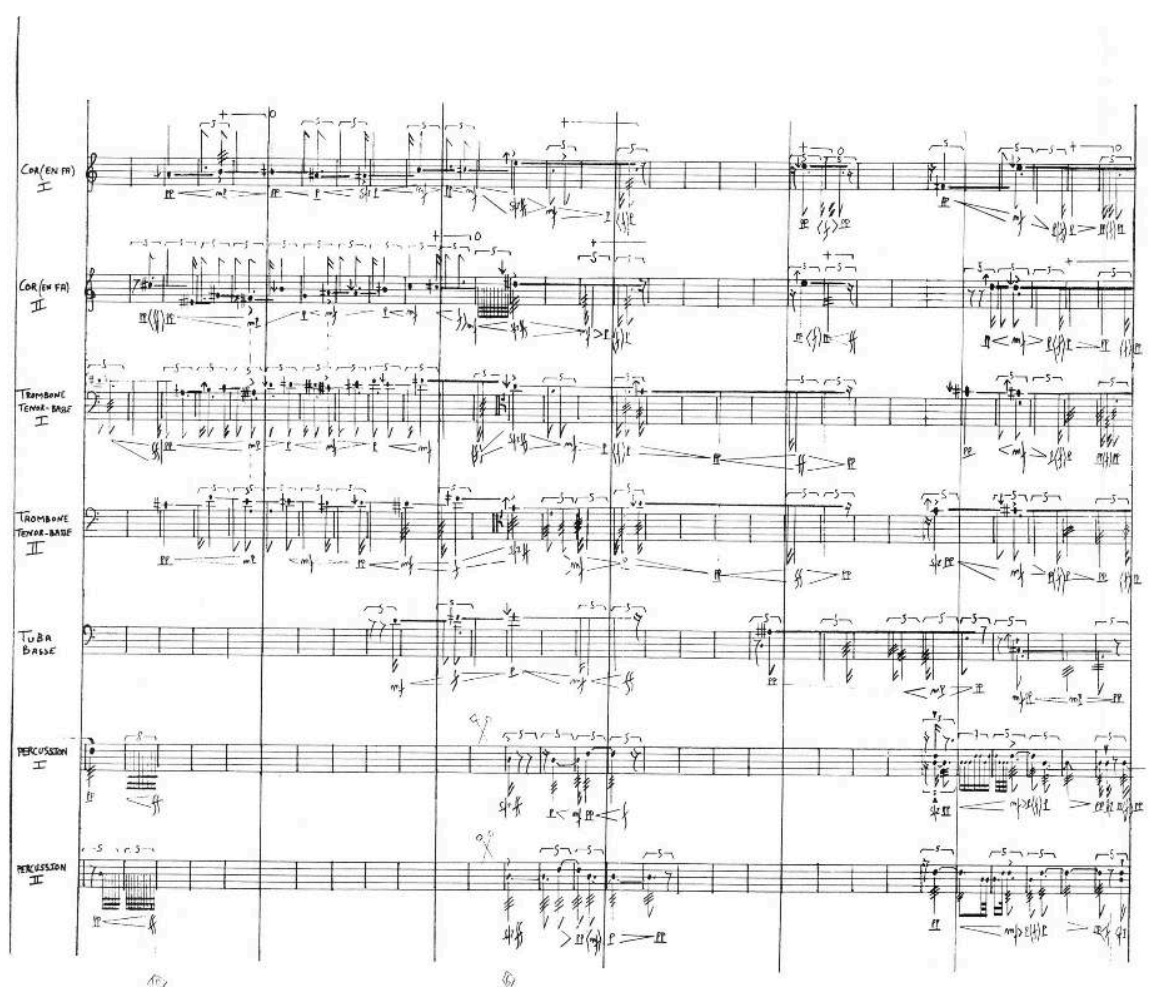

\section{Le compositeur}

1 Né le 27 janvier 1968, il effectue des études musicales au CNR de Caen puis au Conservatoire National Supérieur de Musique de Paris (classe d'Ivo Malec depuis 1988).

2 Il découvre la musique contemporaine très tôt. Les œuvres de Ligeti et Stockhausen seront alors déterminantes pour ses premiers "essais de compositeur".

3 En 1983, il rencontre Ivo Malec pour la première fois. 
4 En 1985, il devient l'élève d'Horatio Radulescu : à son contact, et à la révélation de sa musique (Iubiri pour 16 instruments) il décide d'utiliser le spectre comme élément formel d'un langage harmonico-timbrique.

En 1986, il assiste aux cours d'été de Darmstadt et crée Vitrail pour violon solo. Les relations avec de nombreux interprètes (tels que Levine Andrade, Pierre-Yves Artaud...) et compositeurs (Dillon, Ferneyhough,...) se révèlent extrêment stimulants.

En 1987, il étudie avec Gérard Grisey au Conservatoire américain de Fontainebleau, dont il reçoit les conseils, ainsi que ceux de Radulescu, dans l'écriture de Imiday pour 6 instruments.

En 1988, il retourne à Darmstadt (création de Imiday).

8 En 1987-1988, il est à plusieurs reprises l'invité de Gérard Pesson et Jean-Pierre Derrien sur France Musique. Sont diffusées à ces occasions : Vitrail, Temple. Puis créées : Culte et Alloys.

9 La rencontre d'Elliott Carter (Le matin des musiciens de Jean-Pierre Derrien) en décembre 1988 demeure l'un de ses plus émouvants souvenirs musicaux.

10 Il considère ses études de violon, auprès de Rodrigue Milosi et d'analyse auprès d'Alain Poirier, comme déterminantes dans sa formation actuelle, ainsi que ses contacts avec des compositeurs français comme Lenot, Gaussin, Grisey, Haim, Beaufils, ou anglais : Dillon, Dench, ou encore italiens: Brizzi, Giuliano, comme un terrain très privilégié d'échanges d'idées musicales.

11 Actuellement, il s'attache, dans ses œuvres, à articuler les formes musicales, par une directionnalité des informations micro-et macro-structurelles applicables à toutes les couches simultanées du matériau sonore et à établir des réflexions quant aux interrelations "organiques" entre les composantes du son à partir du spectre.

\section{Le sérialisme}

12 Du point de vue conceptuel, le sérialisme, dans toutes les phases de son développement (viennois, post-webernien,...) nous apparait avant tout comme une tentative d'édification d'un nouveau langage. Le problème majeur ayant été de supprimer le rapport dominante-tonique ainsi que toutes les modulations expressives, et, par là même, de trouver un système aussi efficace que le séculaire système tonal. Le principe des degrés forts et faibles aboli, l'idée de privilégier les enchaînements intervalliques à l'intérieur de la série, puis de privilégier les enchaînements de certaines de ces séries à l'intérieur de l'œuvre, et de déduire ainsi des réseaux de combinatoire et prolifération a, de toute manière, permis d'établir à un moment donné de l'histoire de la musique un nouveau langage. De là sont nées les plus grandes œuvres du XXe siècle, de Webern à Boulez et Stockhausen (et de tant d'autres compositeurs).

Depuis une quinzaine d'années, est née en France une nouvelle tendance, dont l'héritage provient plus directement de Varèse que de Schoenberg, et dont les protagonistes sont: Radulescu, Grisey, Murail. Les préoccupations de cette tendance s'articulent autour du spectre harmonique, et de la microscopie du timbre-son, et se sont manifestées de façon très différentes chez ces compositeurs dans des œuvres fondamentales de cette pensée comme Iubiri de Radulescu; Modulation de Grisey; ou Gondwana de Murail. 
Cela ne nous amène pas à remettre en cause la musique sérielle, certaines données de la musique dite spectrale étant directement ou indirectement héritées de cette musique sérielle (!), mais plutôt à nous interroger sur l'évolution de la musique, et à penser que la musique se renouvelle d'elle-même, au travers des compositeurs, qu'ils s'identifient à l'une ou l'autre des grandes tendance ou qu'ils soient indépendants, tels que Messiaen, Dutilleux ou Malec.

\section{Le concept de musique pure}

15 Dans le cadre d'une "musique à programme", c'est-à-dire utilisant un texte poétique ou même inspiré d'un texte poétique, il semble important d'établir un équilibre perceptible entre l'expressivité de la musique, d'une part, et l'expressivité du texte, d'autre part, afin que l'une et l'autre ne s'annulent pas, mais se "portent" et par là même, se "subliment". L'une des concrétisations les plus fortes de cet équilibre texte/ musique (notamment par une véritable utilisation intrumentale de la voix) se trouve dans le rapport Mallarmé ou Char/Boulez au travers d'œuvres comme Pli selon Pli, Le Soleil des Eaux, ou bien chez Bach, dans une œuvre comme La Passion selon St Matthieu dans laquelle existe une symbolique très forte entre les figurations musicales et le texte biblique.

pourrait de même approfondir un rapport musique/peinture dans le cadre de concerts, certains chefs-d'œuvres picturaux pouvant se révéler sources d'inspiration pour les compositeurs, comme dans le cas très récent de Brian Ferneyhough et de sa pièce pour clarinette et ensemble de 1988: La chute d'Icare, d'après une œuvre de Brueghel.

Il nous semblerait intéressant de développer l'aspect projection de toile sur grand écran pendant le déroulement d'un concert. Le travail avec un peintre pouvant s'avérer très stimulant dans la conception de la mise en scène de ce type de projection.

La concrétisation la plus intéressante, mais la plus difficile à maîtriser, pourrait être la composition d'un opéra; la création picturale, chorégraphique, littéraire devant être parfaitement intégrée à la musique afin de ne pas créer un simple "spectacle", mais réellement une "œuvre d'art" dont le squelette serait la musique.

Un tel projet demande un temps de maturation assez long, de par son importance, mais aussi à cause du choix des artistes et de l'élaboration d'une commune compréhension et articulation de l'œuvre

\section{Fonction de la musique aujourd'hui}

20 La fonction, comme le rôle de la musique d'aujourd'hui, devraient être similaires à ceux de toutes les musiques du passé : un moyen de communication plus profond et plus intense que le geste ou la parole. Cette communication devrait être porteuse d'une émotion intense propre à établir des liens plus étroits entre les hommes. La musique offre un état de plénitude rare, par le fait qu'elle naît d'une expression des plus abstraites : l'agencement des sons.

21 Cette communication s'établit plus difficilement entre le public et les créateurs contemporains. Cela semble être accentué à notre époque, par l'affluence des langages 
et des esthétiques différentes, et donc par le manque d'un outil de compréhension accessible à tous.

Peut-être existe-t-il un problème social dans la communication artistique. Le rythme de vie, l'uniformisation urbaine ne supprimeraient-ils pas un certain désir de culture de la part d'un grand nombre d'individus?

Il existe cependant un noyau (radios, festivals, revues musicales) qui permet à la création de vivre et de se développer aux yeux d'un public croissant et donc de maintenir le rôle, à travers les compositeurs, de la musique comme nécessaire purificatrice de l'âme.

\section{Technique et pensée musicale}

L'une de nos préoccupations principales se situe dans l'intégration du micro-intervalle dans notre langage. Il existe trois principales tendances dans la conception de l'utilisation des micro-intervalles :

- les échelles de 24 quarts de tons, conçues comme une extension de la gamme chromatique, et dont l'utilisation est dérivée d'une pensée sérielle, même si dans la réalisation de l'œuvre, elle s'en éloigne,

- les micro-intervalles "d'expressions" placés dans le discours musical pour établir une certaine "ponctuation" de ce discours,

-et une conception à laquelle nous sommes actuellement tous très attachés : l'utilisation d'échelles calculées sur des spectres harmoniques. Ces échelles de spectres, qui se situent dans la lignée des préoccupations d'Edgar Varèse, ont commencé à hanter les compositeurs, comme nous l'avons déjà dit, il y a une quinzaine d'années. L'électroacoustique, d'une part, et l'évolution des moyens d'analyse du son ont permis d'établir de nouveaux systèmes musicaux et de pensée dont la logique ne repose plus sur une discursivité hachée des événements, mais plutôt sur une exploration des hauteurs et des timbres, à l'intérieur d'un temps plus long, par une microscopie de ce timbre-son.

L'ordinateur pourrait, à l'aide de calculs précis sur ces spectres harmoniques, assister le compositeur dans la recherche de situations musicales insoupçonnées, à la fois sur le plan conceptuel (calcul des hauteurs, des formes), mais aussi instrumental (recherche de combinaisons par des analyses individuelles puis synthétiques des spectres instrumentaux).

Si Radulescu, Grisey, Murail, ont ouvert, par leurs œuvres, des portes nouvelles, il est possible que l'on puisse découvrir, à l'aide de moyens techniques élaborés, des régions vierges de ce nouveau terrain musical, qui n'imposeraient pas une mutation radicale de la pensée et de la pratique musicales, mais qui pourraient être la source d'un renouvellement de cette pensée et de cette pratique.

\section{Musique contemporaine et institutions}

Les institutions musicales traditionnelles peuvent représenter un moyen privilégié d'expression pour les compositeurs, qu'il s'agisse de la création (opéra, orchestre symphonique) ou de l'enseignement (conservatoires), permettant de sensibiliser un plus vaste public à la musique contemporaine. Cependant, cette diffusion de la musique d'aujourd'hui (en dehors des radios, festivals et ensembles spécialisés) n'est permise 
que par la proximité des œuvres du répertoire classique, romantique, et moderne qui assurent une certaine rentabilité au concert.

Il est donc important de considérer ces institutions, non pas simplement comme un lieu de création, mais plutôt comme un lieu de sensibilisation des auditeurs et des interprètes aux œuvres récentes. L'enseignement de la musique se faisant à la base dans les conservatoires, les professeurs devraient jouer un rôle de catalyseurs entre cette musique et les jeunes musiciens, ce qui permettrait d'approcher, d'une part, les techniques instrumentales spécifiques et, d'autre part, les conceptions formelles des œuvres, le problème essentiel étant d'établir une chaîne de communication compositeur-interprètes-auditeurs propre à développer une meilleure diffusion de la musique contemporaine.

\section{Musique d'aujourd'hui et traditions}

29 Le jazz, comme la musique contemporaine, souffre d'un problème de diffusion et n'est écouté quasiment que par des "initiés". En regard à cela, on constate un véritable engouement des foules pour la musique rock, qui est elle surmédiatisée, surécoutée par rapport aux qualités artistiques (assez pauvres) qu'elle possède.

Le rock (en fait les musiques de variétés) serait-il la vraie musique d'aujourd'hui, les compositeurs, les jazzmen seraient-ils des fossiles, vestiges d'une époque lointaine ? Il est probable qu'il faut répondre par la négative, et que l'on a plus à apprendre des polyphonies pygmées, des ragas hindous, des chants arabes, des rythmiques d'Afrique noire, que des mélodies en demi-teintes de certaines pop-stars, qui confondent trop facilement le "business" et l'art.

Malgré tout, il existe de grands artistes, tels que Brel, Piaf, Brassens, qui, par leur "poésie" ont marqué leur génération. Il convient alors peut-être de dire que les musiques idiomatiques populaires sont les musiques du présent, et que les musiques "sérieuses", en tout cas plus inspirées, d'aujourd'hui sont les musiques du futur, sans pouvoir cependant présager de rien.

\section{L'œuvre}

Généralement, l'œuvre naît d'une idée poétique, suggérée par un paysage, une phrase littéraire, un sentiment, la vue d'une œuvre picturale, un événement personnel, et devient par là même, une sorte de reflet de la vie, de l'intimité et de la philosophie du compositeur. Ces "révélateurs"1 sont fortement chargés d'une couleur, d'un caractère déterminants dans le choix des instruments, du déroulement et de l'expressivité de la musique. Presque simultanément apparaissent les éléments structurels de la pièce : les figurations musicales, les déplacements de registres, le choix des hauteurs, des durées, du timbre, de la dynamique, de l'instrumentation en fonction du discours, des points d'impact maximum. A tout cela, suit un long travail précompositionnel fondamental dans l'organisation des événements de l'œuvre.

33 Ainsi, nous procédons d'abord par l'établissement d'une échelle des hauteurs (généralement à l'intérieur de spectres harmoniques, ou de modes spécifiques), puis d'un calcul des proportions et des durées, de la directionnalité registrale, des articulations micro-et macro-formelles du timbre, de l'évolution des différents types 
d'écriture à l'intérieur d'une ou de plusieurs strates de musique, de l'évolution du degré de complémentarité de ces types de musique, des densités harmoniques, timbriques et instrumentales, des courbes dynamiques. S'il est vrai qu'il existe un travail de prédétermination, il n'impose pas une écriture mécanique, mais plutôt il propose un type d'organisation des informations micro-et macro-structurelles indispensable à la composition de l'œuvre ; l'écriture même de la partition étant la phase la plus longue de cette naissance, et dans laquelle se libère le plus l'inspiration. Cette prédétermination intervenant plus sur les grandes articulations, elle peut subir quelques modifications locales dans l'écriture, mais surtout guider l'inspiration, en rapport avec l'idée poétique initiale.

\section{NOTES}

1. Des textes de Bachelard, Bergson, Cioran, Debono, Mann, des toiles de Hartung, Soulages, Dubois, Futura, Hélion ont déjà, dans notre parcours personnel, été des "révélateurs", dans la composition d'œuvres de musique pure.. 


\section{Jacques Demierre}

1 Je ne crois pas pouvoir définir mon propre développement, encore moins utiliser le terme même de développement. Que ce soit dans mon évolution de compositeur ou dans le processus proprement dit de création d'une œuvre, les éléments rencontrés ne s'opposent pas entre eux dans le sens d'un dépassement dialectique qui gommerait les aspects spécifiques de chacun. Tout est là, tout le temps, dans la simultanéité et dans la plus grande des diversités. Les oppositions ne s'annulent pas au profit d'un résultat qui irait au-delà d'elles-mêmes, au contraire, c'est dans l'affirmation des différences que naissent véritablement le sens et la possibilité d'une musique ouverte sur le monde et sur elle-même.

2 Je crois qu'une influence extérieure, qu'une découverte, peuvent être importantes non en tant que telles, mais en tant que révélateurs de zones intérieures cachées. En ce sens, et dans le désordre, Frank Zappa, la pensée Zen, Luciano Berio, Paul Bley, certaines musiques africaines, John Coltrane, la linguistique, John Cage, e.e.cummings, la musique électro-acoustique, la poésie sonore, ont joué pour moi le rôle d'indicateurs de direction à l'intérieur de cette zone de simultanéité, où, au fil de la durée, se révèlent d'autres entités apparemment paradoxales. L'avenir est, par conséquent, omni-directionnel, ou, ce qui revient au même, non directionnel. Il déroule comme à reculons le tapis d'un éternel présent.

3 Je pense que considérer le sérialisme comme unique mètre de la musique de ce siècle est donner à cette tendance historique une importance que le futur relativisera sûrement. Bien qu'il y ait presque autant de sérialisme que de compositeurs sériels, l'idée d'indépendance a toujours été une constante particulièrement forte à l'intérieur de ce mouvement qui n'en était pas un. Indépendance par rapport au temps, à l'histoire, indépendance par rapport à l'espace, à la géographie, ces notions m'ont toujours paru étranges et lointaines. Aujourd'hui plus encore, où la libre circulation de l'information culturelle, qui tend à compresser notre sensation du temps et de l'espace, nous permet de vérifier empiriquement l'interdépendance des phénomènes. Il ne m'est pas possible d'écrire une musique qui ne prendrait pour référent que la seule tradition occidentale. De la même manière que le sérialisme n'était qu'un chemin parmi d'autres, la musique occidentale n'est qu'une réalisation particulière du rapport au monde développé par les hommes à travers toutes les cultures. Et quand je parle d'interdépendance des phénomènes, c'est le lien qui relie les phénomènes dans leur essence qui m'intéresse, et 
non le phénomène en soi. Il n'est donc pas question ici d'exotisme musical, mais bien plutôt d'une sorte de recherche vers ce qu'on pourrait appeler des univers musicaux, qui sont d'ailleurs très difficiles à définir, puisqu'ils représentent souvent le véritable point de rencontre sonore entre une vision à la fois physique et métaphysique du monde.

4 L'émergence d'un travail de réflexion théorique qui accompagne ou suit la création musicale est souvent liée à des conditions historiques particulières. Les problèmes formels de langage étant au centre des préoccupations des compositeurs sériels, ceux-ci ont trouvé dans la théorisation une sorte de justification de leur production sonore : les résultats de leurs réflexions théoriques devenant le contenu même de leur propre création musicale. Il en est résulté une sorte de circuit fermé qui ne pouvait aboutir qu'à une impasse.

5 Je me suis souvent demandé si la musique devait être forcément un langage pour être considérée comme musique. A voir l'écart étonnant qui existe entre la notion de langage prônée par certains compositeurs et celle définie par la linguistique contemporaine, $\mathrm{j}$ ai l'impression que ce problème de linguistique musicale - qu'on le revendique ou qu'on le rejette - cache en réalité la vieille question du contenu musical. Le fait que, pour certains, la musique ne renvoie qu'à elle-même, ou, au contraire, pour d'autres, qu'elle se confonde avec la vie, me rappelle surtout la véritable difficulté qu'ont les compositeurs à formaliser et à définir le contenu de leur propre musique.

6 Alors que la pensée sérielle se prêtait à merveille à la théorisation, aujourd'hui, la réflexion ne se base plus sur un terrain aussi facilement formalisable. Ce qui ne veut pas dire que la réflexion théorique ait disparu. Elle a pris, je crois, une autre forme, qui dépend étroitement de l'objet à formaliser. Comme celui-ci me paraît être essentiellement un objet relevant $d u$ sens, il est fort probable que les tentatives théoriques ne feront que le cerner, sans jamais l'atteindre, si ce n'est peut-être au travers d'actes de foi difficilement communicables, ou, a contrario, de petits jeux formels gratuits.

7 Les questions d'ordre sémantique qu'un compositeur est amené à se poser en découvrant ou en imaginant les relations entretenues par les éléments composant un matériau musical donné sont en fait les mêmes qu'il a à se poser lorsqu'il considère son rapport au monde qui l'entoure. C'est de la présence ou de l'absence de ce lien essentiel, ontologique et utopique, que dépend la sensation d'existence ou de non-existence, au sens propre, d'une œuvre. Paradoxalement, ce lien ne semble se concrétiser que lorsque le processus de composition repose sur une sorte de vide central que la pensée théorique ne parvient pas à englober et autour duquel les sons viennent se stabiliser. C'est l'émergence de ce vide - hors de tout aboutissement purement linguistique - qui, je crois, donne son sens à une œuvre, indépendamment de toute présence ou absence de systèmes, qui ne sont de toutes manières là qu'en tant que moyens pour atteindre cette nécessité, cette urgence intérieure et fondamentale. Face à ce noyau central, chaque son possède une place réservée dans un étroit rapport de temps et d'espace. Ce lieu d'équilibre se trouve en un point où les forces répulsives et les forces attractives qui s'exercent sur les éléments sonores sont en complémentarité absolue. Mon travail de composition consiste essentiellement à atteindre cet état d'éternité de l'instant à l'intérieur d'un processus qui, lui, est en constante expansion. 


\section{Marco Stroppa}

\section{Le compositeur}

1 Pourquoi demander au compositeur de se définir lui-même ? Pourquoi le contraindre à cette opération d'introspection? Son rôle n'est-il pas de créer plutôt que de se pencher sur ce qu'il a été ou qu'il aurait pu être? Je préfère une vision prospective à une attitude perspective, d'autant plus que notre société est constamment en quête de définitions sommaires qui sont ensuite transformées en images de marque: c'est l'image, en effet, qui est rentable. Et ces images, dépourvues désormais de tout contenu significatif, sont répertoriées et classifiées par les spécialistes : voilà les courants dans lesquels insérer les compositeurs, les spectraux ici, les néo-romantiques là-bas, les néostructuraux de ce côté, et ainsi de suite. A chacun son tour !

2 Permettez-moi donc de garder fièrement mon indépendance vis-à-vis des tendances préétablies. Je n'aime pas ces images figées: elles me rappellent des étiquettes commodes. Mais nous ne sommes pas des confitures!

\section{Le sérialisme}

3 J'appartiens à une génération pour laquelle le sérialisme constitue déjà un système faisant partie de notre tradition musicale passée. J'ai pris conscience des mécanismes de fonctionnement à travers l'analyse de quelques œuvres de référence et l'écriture, comme je l'ai fait avec les principaux autres systèmes employés durant notre histoire de la musique, à partir des modes grecs anciens. Je ne vois donc guère de raisons de s'interroger spécialement sur le sérialisme aujourd'hui : pourquoi lui donner autant d'importance ? Est-ce une raison suffisante d'avoir été un des derniers systèmes? Un premier bilan a déjà été dressé par les choix des compositeurs de notre génération. Les faits me paraissent plus convaincants que les débats d'idées.

D'ailleurs, avec l'esprit d'escalier, il est très facile de s'apercevoir que la rupture avec le passé, si fortement accrochée à la pensée sérielle, était surtout un besoin personnel des compositeurs - en partie dû, je crois, au contexte historique dans lequel ils ont commencé à travailler - mais elle n'existait pas dans la partie concrète: ainsi 
Schoenberg inaugure ses nouvelles découvertes avec une confortable suite pour piano, Webern refait des formes classiques de plus en plus conventionnelles, mais les momifie dans un univers spéculaire abstrait d'une froideur glaciale, Boulez écrit des sonates : quelle rupture spectaculaire! De ce point de vue, des musiciens comme Cage, ou Ligeti ou même le Stravinsky du Sacre me semblent avoir remis en cause bien plus radicalement le rapport avec le passé. Mais le concept de rupture n'est-il pas un peu obsolète aujourd'hui ? N'avons-nous pas appris à maîtriser la tradition et à la déchiffrer savamment afin de rendre notre inspiration plus consciente de son action?

Mais au-delà de l'intérêt théorique qu'il peut susciter, tout système musical nouveau doit produire des œuvres de référence, du moins par rapport à d'autres œuvres de la même époque. Je n'ai jamais cru à une musique purement conceptuelle, ni à une justification théorique d'une créativité insuffisante: le résultat sonore me semble toujours une "condition sine qua non" pour tout effort spéculatif. Ainsi, pendant mes études, je lisais méticuleusement les écrits des principaux personnages du sérialisme et j'écoutais en même temps les œuvres qu'ils admiraient tant. Mais, hélas, si mon esprit en comprenait aisément le fonctionnement abstrait, fort simple d'ailleurs, ma sensibilité réagissait très peu à ces musiques. Au contraire, à l'écoute des œuvres atonales de Schoenberg, Berg ou Webern, ou de compositeurs tels que Bartók, Stravinsky ou Varèse, pour ne rester qu'avec une seule génération, elle en était tout enflammée. Si leur démarche était certes moins systématique, elle ne manquait cependant pas de rigueur. Le résultat musical, enfin, me semblait beaucoup plus épanoui et riche. Et pourtant, je trouvais beaucoup moins d'informations à lire sur ces compositeurs-là...

\section{Le concept de musique pure}

6 Qu'est-ce que la musique "pure" ? Est-ce le contraire de la musique "impure" ? Il peut y avoir beaucoup de musicalité dans une pièce de danse sans musique, ou beaucoup de théâtre dans une pièce purement instrumentale : laquelle est la plus pure?

7 En fait, ce qui compte avant tout est le résultat artistique final, pris dans sa globalité. Personnellement, je suis très partisan des spectacles interdisciplinaires quand ils le sont véritablement, c'est-à-dire quand une collaboration réelle s'établit entre les multiples participants au spectacle et quand il n'y a pas de composante artistique constamment subordonnée aux autres. Mais les temps, les modes de production et les lieux sont souvent si différents et parfois incompatibles, qu'un véritable travail d'équipe est aujourd'hui presque impossible. Qui plus est, les opérateurs artistiques ne sont pas encore assez courageux pour prendre le risque et le temps de monter un spectacle interdisciplinaire. Je crains qu'il ne faille attendre longtemps.

\section{Fonction de la musique aujourd'hui}

Composer, c'est ma façon de communiquer mes idées, exprimer mes émotions et vivre mon rôle dans la société contemporaine. 


\section{Technique et pensée musicale}

9 Nous entrons aujourd'hui dans une phase historique d'extrême excitation: c'est la phase de la maturité de la technologie. Non seulement les outils informatiques sont de plus en plus perfectionnés et puissants, mais surtout les connaissances théoriques, notamment scientifiques, que nous avons acquises sont désormais si sophistiquées, qu'elles nous permettent d'explorer le phénomène musical tout entier avec une rigueur et une lucidité sans précédent.

10 L'outil et la connaissance adéquate pour le maîtriser : c'est l'existence de ces deux aspects au même degré de développement qui est extraordinaire et particulièrement réussi en ce moment. Ce n'était pas ainsi, par exemple, il y a trente ans, au point culminant de la musique électro-acoustique: alors, une réflexion compositionnelle extrêmement pointue se heurtait inexorablement à une technologie géniale, mais débutante, et à des connaissances scientifiques insuffisantes par rapport à la demande théorique des compositeurs.

11 C'est surtout l'aspect conceptuel de la maturité qui m'intéresse ici, ce qui n'a rien à voir avec l'exploitation commerciale de la technologie, ni avec le marché des machines à bas prix. En réduisant la complexité du phénomène musical au niveau d'un code MIDI d'une banalité incontestable, ce marché a failli écraser une recherche musicale qui commençait à prendre vigueur. Au contraire, la maturité conceptuelle que j'imagine est dangereuse : elle provoque et bouleverse les attitudes musicales acquises pendant notre formation au conservatoire, nous oblige à nous investir dans des domaines théoriques et pratiques que, hélas, la majeure partie des institutions traditionnelles se refusent encore à enseigner de façon adéquate, nous pousse enfin à revoir complètement les fondations mêmes de notre écriture classique, c'est-à-dire la nature du matériau musical lui-même.

12 Un défi gigantesque nous est lancé en ce moment : c'est aux compositeurs de notre génération de prendre courage et d'en amorcer la réponse. Il faut tout d'abord, c'est évident, maitriser les potentialités et les limites de ce nouvel univers technologique, du moins autant que l'on maitrise le monde des instruments traditionnels. Apprendre à communiquer avec une machine est, en effet, un des moments les plus dramatiques: forcé de réfléchir à son action et de la formaliser partiellement, le compositeur doit secouer son intuition et mettre à nu quelques-uns des mécanismes musicaux qui lui étaient jusque là secrets. Ce faisant, il acquiert conscience de son mode de fonctionnement, ce qui produit, tout naturellement, une phase de réflexion théorique qui, à son tour, alimente à la fois l'intuition et la capacité de formalisation, et ainsi de suite.

13 Ce parcours circulaire est particulièrement frappant dans un domaine parmi les plus féconds, celui de la synthèse de matériaux sonores. Les recherches dans le cadre de la psychologie de la perception, grâce particulièrement aux travaux de Stephen McAdams, ont radicalement changé la conscience du rapport entre écriture et résulat sonore perçu de ceux qui ont su se mettre au courant de ces travaux et en profiter.

En leur donnant les moyens conceptuels pour maîtriser l'écriture et la composition du matériau musical, ces compositeurs ont appris à transformer un objet trouvé, aussi riche soit-il - le matériau des instruments traditionnels - en un objet à composer, le matériau synthétique. Un territoire, jusqu'à ce moment-là vierge, a été ouvert tout 
grand aux recherches les plus hardies. Ainsi, par exemple, nous avons unifié véritablement des concepts tels que "timbre" et "harmonie" en les rendant deux faces de la même médaille sonore. Nous savons transformer n'importe quel accord en un timbre fusionné, ou un timbre en un accord. Pour y réussir avec succès, il faut agir avec une extrême précision sur des paramètres sonores microscopiques, dont la durée de vie se mesure en millièmes de secondes et que seul l'ordinateur nous permet de contrôler. La connaissance de cette mystérieuse alchimie de paramètres qui est le timbre a énormément progressé : quelle distance avons-nous parcourue depuis les premiers textes de Stockhausen!

15 Maîtriser l'outil technologique n'est cependant que la première étape du travail. Une réflexion théorique accrue est ensuite indispensable, non seulement pour rendre ce travail de plus en plus conscient et le libérer ainsi de l'amateurisme d'une infatuation technocratique stérile, mais aussi pour jeter les fondations d'une communication plus riche et engagée avec les interprètes et les auditeurs. Voilà donc une tâche épineuse pour notre génération: réétablir les rapports entre les enjeux ouverts par la technologie et notre tradition musicale théorique et pratique. C'est un peu l'envers de ce qui s'était produit il y a trente ans: nous n'avons pas encore fait un effort de réflexion digne des enjeux avec lesquels nous avons à faire.

Dans l'état actuel des choses, donc, et indépendamment de notre réussite à venir, tout jeune compositeur qui n'aurait pas senti intimement aujourd'hui le besoin de s'investir à corps perdu dans cette aventure musicale et théorique est déjà passé à côté du défi.

\section{Musique d'aujourd'hui et traditions}

Chaque idiome demande tout d'abord une attitude et une écoute qui lui sont propres. Au-delà des sympathies personnelles, il faut donc apprendre à le respecter pour ce qu'il est, sans prétendre qu'il représente autre chose que lui-même. Si la facilité et la vitesse des échanges d'informations courantes nous exposent aujourd'hui à une multitude époustouflante d'idiomes que j'écoute avec plaisir et intérêt, je tiens néanmoins à rester moi-même en tant que compositeur. Je ne veux donc me laisser influencer directement, ni citer quelques-uns de ces idiomes dans mes œuvres, pour la clarté de mon style et de mon... esprit ! 


\section{Philippe Manoury}

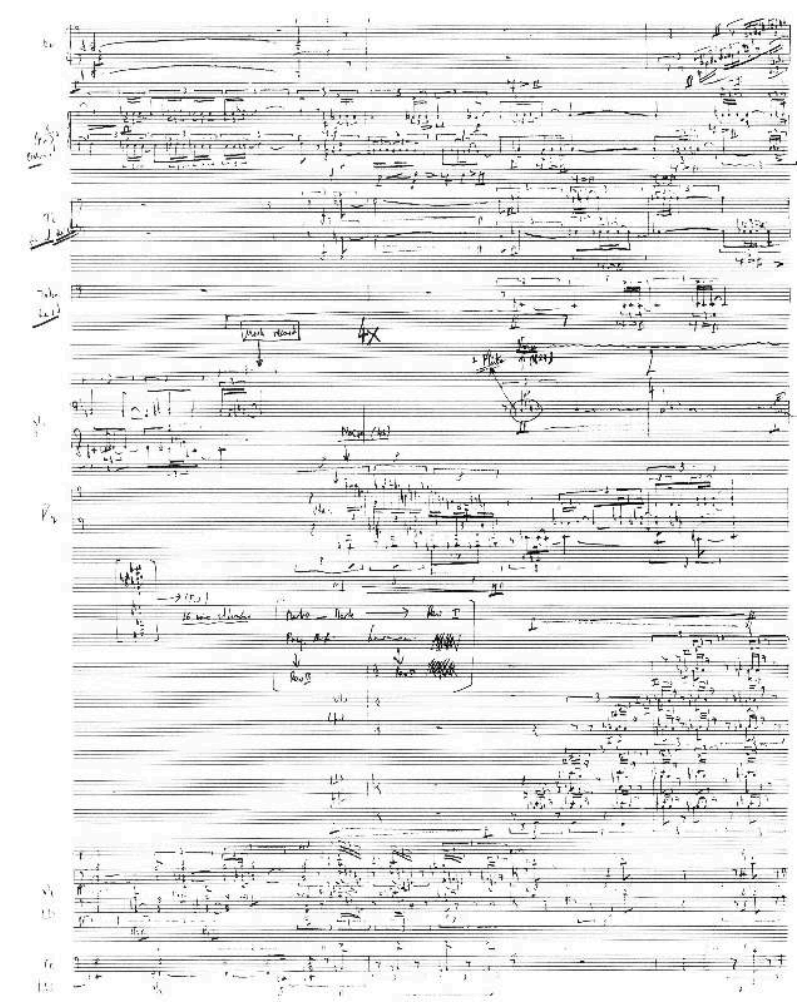

\section{Le compositeur}

1 Mon développement s'est effectué dans la recherche d'une synthèse entre divers courants artistiques qui semblaient autrefois incompatibles. Je citerai comme exemple, d'une part, la démarche issue du sérialisme des années 1950-1960 fondée sur une conception de travail à partir du détail, et celle des conceptions globales et ensemblistes illustrée par des gens tels que Xenakis ou Ligeti.

Le fait de prendre conscience d'une découverte chez autrui et de se l'approprier, j'entends la transformer jusqu'à la rendre sienne, est un acte que l'on rencontre chez tous les compositeurs qui ne se contentent pas d'imiter. 
Une évolution est nécessairement le fruit d'influences et de rencontres diverses autant que de découvertes personnelles. Il est impossible de les répertorier comme de les provoquer. Je peux dire, en ce qui me concerne, que le contact avec les nouvelles technologies a orienté mon travail dans une direction que je n'aurais pas soupçonnée auparavant.

4 Il m'est assez difficile d'envisager l'avenir à long terme. Je citerai volontiers (de mémoire) le propos de Paul Klee: "Je travaille en attendant que survienne quelque chose."

\section{Le sérialisme}

5 Ce que les gens entendent communément par sérialisme me semble avant tout représenter une époque plutôt qu'autre chose, celle des années radicales 1950-1960. Si cette époque était caractérisée par une volonté esthétique propre (celle de la recherche de la discontinuité et de la fragmentation dans le discours), le sérialisme n'en était que l'instrument. Il me semble important de dissiper le malentendu qui consiste à penser qu'il y a une causalité obligée entre moyens techniques et volonté esthétique. Certaines œuvres de Xenakis de cette époque ne sonnent pas très différemment de certaines autres, qualifiées de sérielles.

6 Le sérialisme n'est qu'une technique, non une esthétique. Il me semblerait d'ailleurs absurde de concentrer la vision d'une œuvre sur le système de référence qu'elle utilise, car les décisions que prend le compositeur pour donner un sens à cette œuvre ne sont pratiquement jamais répertoriées sous forme de règles dans le système en question. Ce n'est pas dans un traité d'harmonie tonale qu'on aura l'explication des Variations Diabelli, ni dans le système sériel qu'on aura celle de Gruppen.

7 Et d'ailleurs de quel système s'agit-il ? Le seul concept que le sérialisme a finalement produit est celui de l'absence de hiérarchies a priori entre les douze sons. Ce n'est pas avec cela qu'on fait une œuvre, d'autant que, si je le désire, je peux imposer une hiérarchie quelconque suivant ce que je voudrais exprimer. Le fait que cette hiérarchie n'est plus donnée a priori est le seul lien qui m'attache au sérialisme.

La rupture avec le passé est but toujours salutaire. Seulement, il ne faut pas se leurrer.

9 La génération des années cinquante (pour éviter le mot de sérialisme) a quand même bénéficié de l'héritage de l'Ecole de Vienne, Stravinsky, Varèse, Debussy... Lisez leurs écrits, ils y font sans cesse référence. Seuls peut-être le Stockhausen commençant à superposer des sinus pour ses Etudes électroniques, ou le Xenakis calculant les vitesses de ses glissandis pour Metastasis ont été véritablement en rupture avec le passé. Ces œuvres ont aujourd'hui pour moi le charme de l'aventure des pionniers, mais ne m'atteignent plus guère. Le compositeur allemand a eu, peu de temps après, la clairvoyance d'introduire une voix d'enfant au milieu de ses spectres électroniques. On ne peut pas faire radicalement table rase de ce qui a été. Il fallait le tenter, merci, Messieurs, de l'avoir fait pour nous. Cependant, voyant les divers mouvements néoquelque chose qui apparaissent çà et là, alors oui : la rupture avec le passé est but toujours salutaire.

10 La situation aujourd'hui est très différente. Surtout beaucoup plus complexe. Encore un système unificateur? Je ne pense pas que ce soit à souhaiter. La modernité a la forme d'un archipel, non d'un continent. 
11 Les compositeurs de ma génération sont suffisamment dégagés des pôles des années cinquante (ou du moins devraient l'être) pour tenter de nouvelles expériences. L'atout technologique n'est pas indifférent à cet état de chose, cependant il résout moins de problèmes qu'il ne pose de nouvelles questions. Je vois, tout autour de moi, bon nombre de réflexions théoriques, mais c'est quand leur mise en pratique s'avère fructueuse qu'il convient de s'y attarder.

12 J'apprends à relativiser les choses malgré tout, il y a ceux qui donnent le maximum dans leur jeunesse (voir Berlioz et Schoenberg) et ceux qui ont besoin de l'expérience pour donner ce maximum (voir Wagner et Debussy). Plus encore, il y va du temps comme des religions, il lui faut des sacrifiés. Il y a des générations qui ont pu travailler au bon moment, et ceux, par exemple, qui ont eu vingt ans en 1914... C'est indépendant des qualités personnelles de chacun mais cela compte. Oui, je le pense très fortement: les compositeurs de ma génération devraient être suffisamment dégagés des pôles des années cinquante pour tenter de nouvelles expériences.

\section{Le concept de musique pure}

13 Comment appellerait-on le contraire de "musique pure" ?

14 Pour moi, la musique renvoie tout le temps à quelque chose d'autre, mais elle doit avoir aussi son intérêt en elle-même. C'est la différence qu'il y a entre Verdi et Wagner, les deux étaient animés d'un génie dramatique, mais à part cela, le premier n'a pas grandchose à me dire lorsque je cherche une richesse de conception harmonique ou orchestrale vraiment remarquable.

La musique liée au théâtre dans ces dernières années a trop souffert de ce manque de qualité interne. J'entends parfois dire: "L'univers de M. K. est proche de celui de Beckett par la dérision", je réponds: "Vous avez raison de dire par la dérision car je voyais un abîme entre les deux."

16 Un principe réunit tous les courants artistiques à mon sens : celui de la simulation. Nous ne décrivons pas de vérités, mais nous les simulons le temps d'une œuvre. C'est un besoin, non un jeu.

17 Je suis extrêmement attentif aux autres courants artistiques (il n'y a guère que cela qui m'intéresse) et j'y puise bon nombre de mes idées. Celles-ci sont alors méconnaissables, car on ne peut impunément passer d'une catégorie à une autre. Seul le concept demeure, et personne ne s'aperçoit de rien.

\section{Fonction de la musique aujourd'hui}

18 J'aime les œuvres qui en disent long sur ceux qui les ont écrites. Un artiste m'intéresse à partir du moment où il me parle de lui. A part cela je ne sais pas si la musique a une fonction sociale. Si elle devait en avoir une, j'aimerais que ce soit celle de montrer que l'imagination demeure "la reine des facultés". 


\section{Technique et pensée musicale}

19 Les principes dits "naturels" de résonance ne me préoccupent guère, ils renvoient à des formes trop stéréotypées, je préfère que l'organisation que je propose, finalement aussi arbitraire que les autres, semble naturelle pendant le temps où elle se déroule. Il n'y a pas pour moi de relation univoque entre le monde physique et notre imagination. Elle aussi fait partie de la nature.

20 Les échelles de hauteurs ne sont plus un fondement de base de la composition mais une déviation par rapport à une norme. En d'autres termes, les quarts, sixièmes, huitièmes de tons, etc., n'ont pour moi aucune valeur propre, il m'est peut-être arrivé de les utiliser sans le savoir, dans le passage d'une échelle tempérée à une autre qui ne l'est pas.

21 La recherche de nouveaux matériaux est, par contre, un des points fondamentaux de l'activité musicale actuelle. Elle va de pair avec celle de nouvelles manières de traiter ce matériau. On ne compose pas de la même façon suivant que l'on utilise un matériau non traditionnel ou les sons instrumentaux. Dans le dernier cas, nous possédons un savoir empirique et l'imagination fonctionne sur nos connaissances. Dans le premier, l'imagination est nourrie par l'expérimentation. Ce qu'il y a de passionnant dans cette démarche c'est le fait que l'on trouve les modes de développement de ces matériaux en fonction de leur poids et de leur morphologie. Ce ne sont plus des types de traitement plus ou moins abstraits et indépendants des objets (comme les transpositions, permutations, etc.) qui peuvent faire l'affaire, mais des cas que l'on découvre dans l'expérimentation. Le matériau et la structure peuvent se confondre parfois.

22 Je ne fais pas de la musique expérimentale, mais prends le stade expérimental comme un moment de la composition. C'est celui où l'on découvre et où l'on se familiarise avec le matériau. Ensuite, seulement ensuite, on conceptualise, met en forme.

\section{Musique contemporaine et institution}

Les Centres de recherche. Ils sont assez nombreux, et, Dieu merci, entretiennent entre eux des rapports courtois. Ce qu'il faut maintenant, ce sont des sociétés de production et de diffusion musicale pour dégager ces centres de recherche qui ne peuvent pas toujours assurer les deux fonctions à la fois avec le même tonus. L'IRCAM, où je travaille régulièrement, a, entre autres, le privilège d'être un lieu de rencontre international, et de ne pas défendre, quoi qu'on en dise, une esthétique précise. Lorsqu'on cherche à dégager une esthétique à partir d'un instrument, c'est, ou bien que l'instrument est trop limité, ou bien que l'esthétique est pauvre.

Les Conservatoires. Ils conservent, à quelques exceptions près. En voici une. J'enseigne la composition et l'informatique musicale au CNSM de Lyon. On m'a dit de faire ce que je voulais. Nous sommes deux professeurs (avec Denis Lorrain), un ingénieur donnant des cours d'informatique, et un autre initiant aux techniques du son. Les élèves sont sélectionnés sur leur seule maturité musicale. Ils ont entre leurs mains un matériel comparable (en puissance) à celui de l'IRCAM sur lequel ils peuvent travailler jour et nuit. En trois ans, ils doivent tout maîtriser, écriture instrumentale et moyens technologiques. Ce seront des compositeurs au sens moderne du terme. Cela n'a rien à voir avec le talent, mais lui, on ne l'enseigne pas! 
La Critique. Je parle de la critique spécialisée, musicologique. Rien encore. On croule sous les analyses des compositeurs qui avaient notre âge à une autre époque, et dans lesquelles on n'oublie pas une double croche. Peut-être parce qu'un musicologue se doit d'être plus jeune que l'objet de son étude ? Donc, patience!

\section{L'industrie}

Celle du disque ne tourne pas très rond en ce qui nous concerne.

A l'IRCAM, nous travaillons sur de nouveaux prototypes de machine. J'aimerais qu'une société s'y intéresse pour que ces prototypes cessent de l'être.

Le sponsoring est encore balbutiant en France, mais il progresse. Il est vrai qu'on rencontre plus d'industriels intéressés par la sculpture et la peinture que par la musique car la valeur marchande y est plus évidente. Quand ils subventionnent de jeunes artistes, c'est plus souvent pour jouer des quatuors de Brahms (grrr...) que de la musique nouvelle. Cela fait plus chic pour la clientèle. Il faudrait les encourager maintenant à prendre quelques risques, ils pourraient peut-être s'en enorgueillir avec raison plus tard.

\section{Le Ministère. Pourquoi y a-t-il autant de compositeurs dans ses murs?}

Les orchestres. De nombreux problèmes. Le temps accordé aux répétitions est souvent dérisoire si on le compare avec celui accordé au répertoire traditionnel. Il y a peu de politique cohérente au niveau de la diffusion. On fait des créations, et puis plus rien. Cela fait quinze ans que l'on entend cela, mais tant pis, on continue. Il n'y a guère que dans les “maisons spécialisées" où l'on rencontre un effort dans ce sens.

La radio. On fait des efforts. Certains producteurs cherchent à savoir ce qui se passe. Cependant, il devrait y avoir plus de coordination entre les manifestations "live" et les programmes de radio qui feraient une pédagogie adaptée à chaque cas de figure.

31 La SACEM. Le jour où elle a décidé de prélever de l'argent sur la musique commerciale pour subventionner la création contemporaine est pour nous identique à celui où Léon Blum accordait les congés payés aux ouvriers (c'est-à-dire qu'on ne revient pas sur ces choses-là). Cependant, il veillait, lui, à ce que les salaires aillent vraiment à ceux qui avaient fourni le travail. Il n'était pas plus honnête, seulement mieux organisé.

se concert. Notre musique va souvent dans le sens dune inadéquation avec les cas de figures traditionnels telles que les dispositions classiques de l'orchestre, de la scène et du public. Les problèmes d'exécution en sont pour autant complexifiés. On devrait faire plus d'effort pour investir des lieux qui ne sont pas initialement prévus pour la musique, quitte à en traiter la partie acoustique.

La télévision. Qui se soucie de ce que veulent dire exactement les compositeurs de notre génération? Il n'y a guère que dans les télévisions étrangères (USA ou GrandeBretagne) où j'ai pu répondre à des questions différentes de : "comment fait-on pour gagner sa vie en tant que compositeur?" 


\section{Musique d'aujourd'hui et traditions}

34 Lorsque je n'écoute pas de musique dite "classique" ou dite "contemporaine", c'est vraiment vers les musiques extra-européennes que je me tourne. Cela nettoie mes oreilles. J'y puise pas mal de mes idées, surtout en ce qui concerne les principes généraux (phénomènes rythmiques, conception dynamique du son...). Ensuite, c'est comme toujours. C'est tellement transformé qu'on ne voit pas. Je ne déteste rien tant que l'exotisme musical.

35 J'ai fait, comme tout compositeur contemporain, un voyage à Bali (j'en connais un, et un grand, qui n'y est jamais allé cependant). Les musiciens que j'y ai vus font des schémas rythmiques que n'arrivera jamais à réaliser aucun musicien occidental. La musique, là-bas comme dans beaucoup de pays où la tradition occidentale ne s'est pas implantée, n'est pas un métier de spécialiste. C'est admirable. Chez nous, c'est très différent. C'est admirable aussi.

36 En Europe occidentale, on a oublié notre folklore. C'est peut-être dommage mais cela fait bouger les choses. Alors ce n'est pas dommage.

37 J'ai de l'admiration pour certains musiciens de jazz ou de rock. Cependant la plupart du temps cela m'ennuie. Je pourrais expliquer pourquoi, avec des détails, mais je ne convaincrais personne. Il en va de ses goûts comme de ses passions : ils sont souvent au-delà de la raison.

\section{L'œuvre}

38 Je ne saurais dire comment naît l'œuvre, c'est trop variable. L'idée de départ peut être confuse et s'affiner lors du travail, ou au contraire être très claire et dévier tellement qu'elle fait place à une autre. J'ai tendance à travailler de plus en plus localement. J'aime expérimenter, voir ce que cela donne. Je trouve très belle la définition que François Truffaut donnait d'Orson Welles: "il filmait comme un exhibitionniste, et montait comme un censeur". En règle générale, mes gommes s'usent plus vite que mes crayons. 


\section{Jean-Claude Eloy}

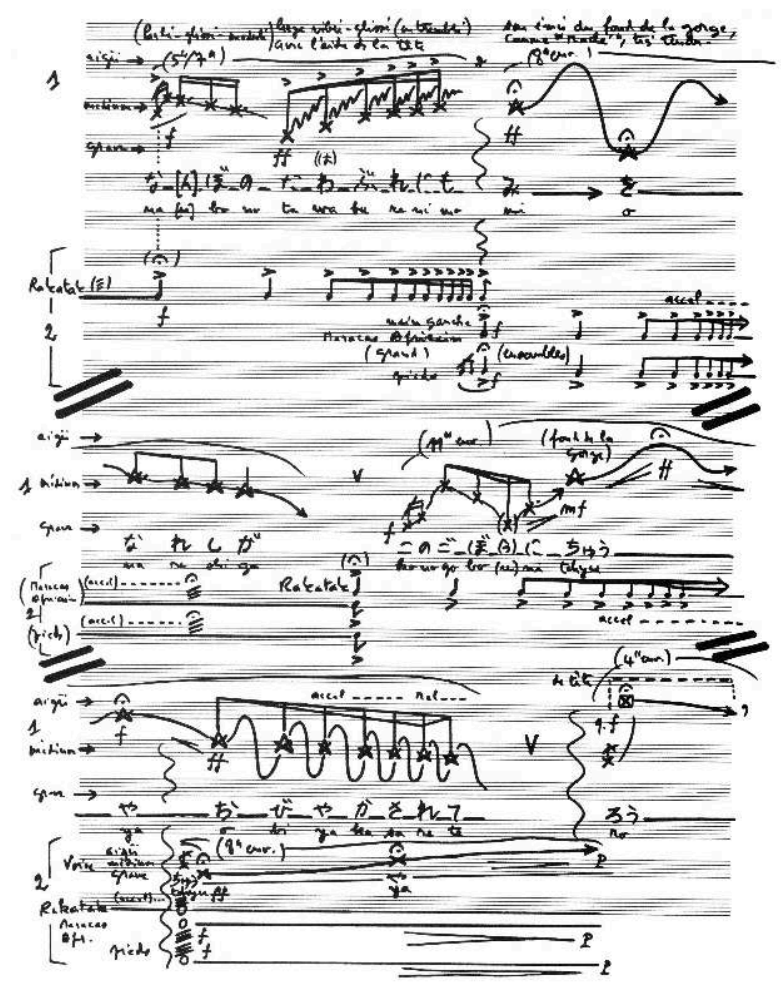

\section{Le compositeur}

1 Mon travail de compositeur forme actuellement un catalogue de 39 numéros : du n ${ }^{\circ} 1$, Nocturne, pour piano, 9', 1954; au n 39, Anâhata, pour cinq musiciens traditionnels japonais, un percussionniste (avec un orchestre de percussions), électroacoustique (réalisée avec l'aide de quatre studios européens), 215', 1984/1986.

2 Entre les deux, quelques titres : Equivalences (18 instruments, 10); Faisceaux-Diffractions (28 instrumentistes, 25'); Kamakala ( 3 orchestres, 5 groupes de choeurs, 3 chefs, 30') ; Shanti (électroacoustique, 140'); Gaku-no-Michi (électroacoustique, 240), Yo-In (percussions, électroacoustique, 220'), etc. 
Bout à bout, ces œuvres représentent une continuité de 24 heures de musique environ. Pratiquement, il est impossible aujourd'hui de trouver la moindre trace de ces heures dans la société ; tant sous la forme de reproductions graphiques (partitions) que sous une forme mécanique (disques/cassettes), et pas même sous la forme d'un livre! Dans ces conditions, comment et de quoi pouvons-nous parler?... Si je commente la technique de modalité chromatique qui contrôle toute l'écriture de Kamakala, de Faisceaux-Diffractions, de Fluctuante-Immuable, qui aura les moyens de voir exactement à quoi je me réfère? Si je mets en lumière l'esthétique du crescendo généralisé, telle qu'elle se réalise, sous deux formes différentes, dans A l'approche du feu méditant et dans Kamakala, qui sera en mesure de pouvoir apprécier la différence? Si j'analyse la forme directionnelle multiple, telle qu'elle se manifeste dans Shanti, par rapport aux formes directionnelles uniques qui précèdent dans ma production, qui aura accès aux données nécessaires pour saisir de quoi je parle? Si j'explique la parenté entre les techniques statistiques d'écriture, telles qu'elles apparaissent dans Le cheminement à travers les mondes ( $3^{\mathrm{e}}$ partie de $A$ l'approche $d u$ feu méditant), et les techniques de même nature utilisées à la fin de Fluctuante-Immuable, qui pourra suivre concrètement ce propos ? Si je m'étends sur la structure dramaturgique de Gaku-no-Michi et les rapports dialectiques entre textures abstraites et textures concrètes, qui entendra dans sa tête à quoi je me réfère ? D’autres thèmes pourraient être développés :

- Transformations des fonctions de la percussion, de Polychromes à Yo-In.

- Chant Shomyo : technique, notation, récréation, dans A l'approche du feu méditant et Anâhata.

- Le studio électroacoustique comme multiplicateur d'une source instrumentale : $3^{\mathrm{e}}$ partie de Anâhata (Nimilana/Unimilana).

- Equivalences : une œuvre post-boulézienne?

-... etc. Ce petit jeu pourrait continuer à l'infini.

Si je commente Mozart dans un article, tout le monde (vos lecteurs, votre rédaction, etc.) comprendra et suivra mon commentaire, parce que nous aurons autour de Mozart une expérience vécue, commune. Même jusqu'à Stockhausen, je peux utiliser le commentaire verbal avec une chance d'être entendu des spécialistes. Mais si j'écris sur ma propre évolution de compositeur, ses étapes, ses métamorphoses, ses permanences, etc., non seulement cela débordera largement le cadre d'une réponse à une enquête, mais, en plus, nous n'aurons plus que l'imaginaire et les abstractions comme références, puisque nous ne pourrons pas partager - même entre spécialistes - une expérience commune vivante. Et je suis certain qu'il nous est impossible de partager quoi que ce soit par les mots sans avoir vécu, au moins une fois: Gaku-no-Michi, Shanti, Yo-In, A l'approche du feu méditant, Anâhata, etc.

5 J'illustre mon propos par une petite anecdote. J'ai donné, jusqu'à ce jour, exactement cent conférences (la grande majorité en Amérique et en Asie), centrées de manières diverses autour de mon travail de compositeur. L'une d'elles avait lieu à Madras, en Inde, devant un auditoire de professeurs et élèves en musique karnatique. Les magnétophones refusant obstinément de démarrer, je me voyais obligé de parler deux heures sans aucun exemple audible! Avant de commencer, je demande: "Que savezvous de la musique moderne occidentale ?" Réponses : "Beethoven... Brahms...". Ils ne connaissaient ni Debussy, encore moins Schoenberg, Bartók, etc. J'essayai alors de faire un exposé aussi clair que possible sur les différences de natures entre le processus historique fortement "statique" de l'évolution de la musique (traditionnelle) en Inde et en Asie, et les processus "dynamiques" de l'histoire occidentale (remise en cause de 
l'héritage, du maître, etc.). Successivement, j'expose le principe des modulations accumulées qui mènent à l'atonalisme; la progressive dilution de la fonction thématique ; la libération du rythme par l'évolution vers les valeurs irrationnelles, etc., jusqu'à une explication aussi concrète que possible des nouveaux instruments, menant, bien sûr, à une description générale des outils et des manipulations de "sons complexes" dans les studios électroacoustiques. A la fin, je me livre au rituel des "questions" de l'auditoire... Un homme se lève et me dit : "Could you sing a little bit of your electronic music, so that we could understand the melody of it ?..." ("Pourriezvous nous chanter un peu de votre musique électronique, afin de pouvoir comprendre sa mélodie ?..."). Très embarrassé, je tente d'expliquer à cet homme combien cela est impossible; que la nature des processus électroacoustiques engendre des sons pratiquement inimitables par ma voix, etc. Mais, tout en parlant, je voyais ses yeux indiens devenir de plus en plus noirs, et je sentais ce qui se passait dans sa tête : "quel est donc ce bonhomme qui vient nous parler pendant deux heures de musique, et qui n'est même pas foutu de nous chanter la sienne !..."

Depuis cette aventure cocasse, je considère que le "mot" en musique, n'est rien, et que l'abus qui en est fait aujourd'hui, dans la musique contemporaine, ne peut que mener aux incompréhensions et aux absurdités.

\section{Le sérialisme}

7 Cette question me semble posée de manière assez académique : elle place les problèmes exactement à l'envers! Ce n'est pas la "réflexion" qui décide des "œuvres" : ce sont les cuvres qui ont toujours décidé de la réflexion! La vraie question est: "Y a-t-il actuellement des œuvres musicales qui peuvent se prêter aux types de spéculations théoriques qu'un très petit groupe d'œuvres a engendrées dans son sillage, il y a trente ans ?". Les analystes universitaires, les musicologues, les commentateurs spécialisés, sont-ils capables, dans l'état actuel de leur connaissance, et face à des musiques fondées avant tout sur un travail de masses de timbres et de textures pratiquement "inquantifiables" (donc travaillées avant tout en actions "qualifiables"), d'analyser et de théoriser de la même manière que dans les années cinquante (années fastes pour les analystes, écrivains, et commentateurs de musique) ? Leur discrétion face aux grandes pièces électroacoustiques, par exemple, n'est-elle pas révélatrice de leurs manques, lesquels sont alimentés par l'absence de documents publiés, exploitables ?... Combien y a-t-il eu d'œuvres réellement sérielles dans l'histoire? Les grands créateurs de la génération d'immédiate après-guerre ayant eux-mêmes dépassé leurs premières œuvres sérielles, pourquoi devrions-nous encore nous poser une telle question? Dans un article récent consacré à Stockhausen, je rappelle qu'en 1963 il nous disait, à Bâle : "la série n'est rien de plus qu'une simple proportion de référence pour le matériau: c'est comme le modulor de Le Corbusier; rien de plus." Je rappelle également ce qu'écrivait Boulez pour introduire nos œuvres, dans le programme du Domaine Musical de la saison 1964/1965, en présentant ma génération. “... pour elle, la composition musicale se manifeste naturellement sous certaines formes qui, il y a quelques années seulement, s'abordaient au prix d'un certain nombre de gestes négatifs. Ceci peut nous rassurer sur un fait certain : nous n'arriverons jamais au bout de l'inconnu !" Je souligne exprès ce dernier mot; il est évident que, pour une certaine musicologie moderne actuelle, il est plus aisé de se manifester encore dans le "connu". (Je signale au passage 
mes anciens articles : "Héritage et Vigilance"; "Pour qu'aujourd'hui se multiplie"; "Dix ans après, qu'en reste t'il ?"; "Du côté des disciples"; "Musiques d'Orient: notre univers familier", etc. Mais j'oubliais : ils sont introuvables, comme le reste...)

\section{Le concept de musique pure}

8 Cette idée est fausse et très dangereuse, et ceux qui prétendent à cette "pureté" sont rarement de véritables saints, mais trop souvent des gens incomplets, un peu malades, qui refusent d'admettre qu'ils sont modelés malgré eux, à leur insu et dans leurs actes, par toutes les choses du monde qui les entoure!

Il est vrai que l'histoire de la musique occidentale a produit parfois des perles rares, dites "pures", qui sont des sommets : l'Art de la fugue, les Quatuors de Beethoven, la $2^{\mathrm{e}}$ Sonate de Boulez, etc.

Mais peut-on détacher entièrement l'Art de la fugue de la religion qui est celle de son auteur? Les Quatuors de Beethoven (le $15^{\mathrm{e}}$ entre autres) ne sont-ils pas nourris par l'expressionnisme romantique naissant ? La $2^{\mathrm{e}}$ Sonate de Boulez n'est-elle pas soutenue par une inquiétude, une angoisse, une violence sourde, caractéristique d'une individualité, d'un "caractère", mais aussi d'une époque : celle des années cinquante, à peine sorties de la Seconde Guerre mondiale, et qui se préparaient quasiment à un troisième affrontement général, dans l'atmosphère de la guerre froide ?

D'autre part, l'Art en tant que totalité; dans lequel musique, voix, texte, rituel, représentation, dramaturgie, danse, vue, décor, etc., sont mêlés en un ensemble cohérent, a toujours été un grand idéal de l'humanité, dans tous les continents, qui a créé des sommets inégalés : Parsifal, Tristan, Pelleas, Orfeo, Boris, etc.; le Kathakali en Inde, le théâtre Nô au Japon, le Gamelang dansé de Java, et tant d'autres encore...

Que signifie cette "pureté" ? Ce mot même me gêne : il me rappelle trop l'inquisition, la dictature, tous les totalitarismes, qui commencent toujours par se nourrir des "puritanismes".

\section{Fonction de la musique aujourd'hui}

13 Pourquoi la musique d'aujourd'hui poserait-elle plus de problèmes et d'interrogations que celle d'hier? Quelles sont les "fonctions" de la musique, depuis que le monde est monde, et en dehors des marches militaires ? Enumérons :

- Religion (célébration, rituel, etc.).

- Dramaturgie (œuvres scéniques, fonctionnement synchrone avec une représentation, etc.).

- Divertissement (concert) ; large partie de la musique actuelle, destinée au concert pris comme seule finalité (donc issue du "divertissement social"; ce mot ne signifiant pas forcément "frivolité").

- Fonctions actives de la vie (musiques de travail, pour les champs, sur les bateaux, sonneries militaires, signaux de communications, etc.).

- Possession/Magie (musiques de guérisons, de malédictions, pour lutter contre les esprits, pour convoquer l'esprit, etc.).

L'un des problèmes de la musique contemporaine est celui de sa destination exclusive à une formule stéréotypée du concert; de sa séparation fréquente d'avec les fonctions de 
célébrations, de rituels, de dramaturgie. Mais comment classer une symphonie de Mahler : divertissement? religieux? dramatique?

\section{Technique et pensée musicale}

15 Cette histoire de la résonance naturelle est une vieille lune qui a servi bien des fois à couvrir l'argumentation réactionnaire occidentale. Malheureusement pour ces derniers, toute la musique occidentale classique et romantique repose elle-même sur une abstraction située assez largement en dehors de la résonance naturelle : l'échelle tempérée. Il n'y a pratiquement que ceux qui cherchent à produire correctement des intervalles naturels qui peuvent se référer à cette théorie : les musiciens classiques de l'Inde ; les groupes spécialisés qui essaient de reconstituer la musique médiévale.

Souvenons-nous des premières pages du magistral Traité d'Harmonie de Schoenberg, dans lequel il démontre que tout est dissonance, hors l'Accord Parfait Majeur (non tempéré, devrions-nous ajouter). L'Accord Parfait Mineur, l'Accord de Sixte et Quarte, etc., sont considérés comme dissonances complètes, démonstrations à l'appui.

Si l'on voulait être fidèle à cette théorie un peu "écologique" de la primauté de la résonance naturelle, il faudrait aller très loin dans l'exploitation des micro-tons, combinés avec des intervalles plus exacts. Relisons l'ouvrage de Alain Daniélou : Traité des musicologies comparées. Il démontre que tous les systèmes ont un certain degré d'abstraction par rapport à cette fameuse "résonance" : “...Tous les rapports sonores possibles sont compris théoriquement dans la série indéfinie des harmoniques. Il n'existe donc pas de combinaison harmonique ou mélodique qui ne soit impliquée dans la structure même d'un son unique, puisque la série des nombres..." ; “... le demi-ton mineur $(24 / 25)$ est le rapport du vingt-quatrième harmonique au vingt-cinquième harmonique... le demi-ton tempéré peut être exprimé par l'intervalle de $1000000000^{\mathrm{e}}$ au $1059463094^{\mathrm{e}}$ harmonique..." (je laisse cette affirmation à Daniélou : j'avoue ne pas avoir refait le calcul !) ; “... de nombreux harmoniques apparaissent déjà dans l'étendue des fréquences audibles. En partant d'une note donnée, la troisième octave ne contient que quatre harmoniques différents, mais la huitième en contient 128 et la seizième $32768 . .$. ” ; “... l'idée de diviser l'octave en douze parties est dérivée d'une gamme non tempérée qui était celle de la musique occidentale à une certaine époque. Pourtant, une fois que nous abandonnons le principe de la consonance, il ne semble pas qu'il y ait la moindre raison valable pour préférer une division en douze plutôt qu'une en dix, en onze, en quatorze, en vingt et un. La division de l'octave en sept intervalles égaux avec des tiers de tons... pour obtenir l'échelle de vingt et un sons... donne un admirable langage musical. Cette division constitue l'ancienne "gamme céleste" des Hindous qui reste la gamme de base de la Thaillande, du Laos, du Cambodge, aujourd'hui encore...".

Pour ce qui est de "l'Ordinateur" : il est grand temps de faire tomber les vues utopiques et mythiques. Tous les esprits scientifiques sérieux vous expliqueront que cet outil s'est développé pour élargir et prolonger les capacités de l'homme. Aucun problème d'art, d'esthétique, de communication qualitative et d'expression humaine n'est résolu par la simple apparition de ce type d'outillage ; lequel se transforme chaque année, et ne produit, le plus souvent, que des successions de prototypes. Si les musiciens (comme toujours pernicieusement totalitaires) voient l'homme devenir mutant à travers le seul usage de ces outils, cela n'est simplement pas sérieux! Je remarque que les endroits artistiquement les plus conservateurs, franchement réactionnaires, ou plus simplement 
qui refusent de considérer l'art moderne comme un "art" mais le considèrent comme une "recherche" (!) sont les endroits qui tolèrent toujours "l'ordinateur" comme seul critère de "modernité artistique"! Curieuse rencontre... Les années soixante ont vu naître des grandes quantités de petits studios analogiques dans les universités américaines : qu'en est-il sorti ?... La recherche de moyens et matériaux nouveaux sera toujours nécessaire et utile ; mais pas l'idéologie de la "recherche pour la recherche", qui n'a pas de projet artistique véritable. On a voulu reprendre à la Bourgeoisie Industrielle comme au Marxisme l'idéologie du "Progrès" en l'appliquant à l'Art: les phénomènes artistiques sont d'une autre nature...

\section{Musique contemporaine et institutions}

19 L'Institution est l'une des maladies du monde musical, à l'échelle mondiale, et tout spécialement à l'échelle française. En 25 ans d'activités, je n'ai constaté, autour de l'Institution française, que crises politiques, ambitions dominatrices, désir de pouvoir, malveillances, manœuvres et contre-manœuvres de couloirs; c'est-à-dire du gâchis bureaucratique d'énergies, et des quantités de faux problèmes.

20 Cette maladie vient directement de l'absence quasi totale d'un marché économique pour la musique moderne! Ce vide, ce manque, qui asphyxie tout, est alors compensé par une position de repli : la création d'institutions comme masques et comme béquilles à l'absence d'existence réelle d'une musique, dans une société. Et les "réformes" n'ont jamais rien changé à cet état de fait! Même les institutions "modernes" et nouvellement fondées ont engendré, dès la départ, ce bureaucratisme allié au "m'as-tuvu", qui révèle (psychanalytiquement) l'existence de la maladie. L'Institution en arrive aujourd'hui à mépriser le mot "artiste", pour le remplacer par celui de "chercheur"!... On ne réforme pas une maladie : on la détruit, ou on en meurt !

21 Les seuls organismes qui contribuent à aider l'art musical actuel sont les organismes de productions : certaines radios (hélas pas toutes!); certains festivals. Les festivals sont aujourd'hui, pratiquement, les seules structures qui contribuent (aussi efficacement qu'ils le peuvent, et malgré leurs limites) à faire naître des œuvres.

\section{Musique d'aujourd'hui et traditions}

22 L'ensemble de mes œuvres, et plus particulièrement Kamakala (dans une première phase), et ensuite A l'approche du feu méditant et Anâhata (dans une deuxième phase) constituent la plus claire des réponses... Il me semble (très objectivement, et en observant le paysage autour de moi) que je me suis plus particulièrement avancé dans cette direction, au point même, aujourd'hui, d'être pratiquement tout seul au regard de mes confrères; tout en étant compris sans problème par certains organisateurs, et une partie du public...

23 L'interfaçage et la synthèse entre des sources "anciennes" d'autres civilisations ("recréées", détournées) et la technologie "moderne", qu'offre une œuvre comme Anâhata, n'a pratiquement pas d'équivalent, non seulement en Occident, mais également en Asie; écrasée par l'imitation, trop souvent stérile et académique, de l'Occident. Voilà des années que je m'exprime sur la nécessité de sortir de la seule idéologie de "l'avant-garde", et de nous élargir par le contact et la réflexion auprès des 
musiques plus éloignées: musiques différentes et passionnantes, dont je souhaite profondément la survie, face au laminoir des musiques médiatiques occidentales.

J'ai été maintes fois accusé d'aller chercher des parfums exotiques et des "épices", à une époque où l'artiste se doit de vivre la multiplicité des cultures, des racines... Le provincialisme qui règne encore sur une partie de la musique dite "avancée" m'a toujours étonné. Chacun se toise avec suffisance; les certitudes acquises et l'arrogance tiennent lieu d'ouverture de l'esprit et de culture véritable; on vit à l'aube du XXI siècle avec des traces de la mentalité du XIX . On continue à développer des polémiques entre telle ou telle petite fraction de "l'avant-garde" (?), et on ne veut pas voir combien tout cela est isolé du monde. La véritable "ligne de résistance", le véritable combat, à l'échelle mondiale, aujourd'hui, est celui qui sépare, d'un côté, toutes les musiques non standardisées, quelles que soient leurs origines (contemporaines ou traditionnelles), et de l'autre, l'immense tissu des musiques commerciales standardisées, omniprésentes dans les consciences, et qui recouvrent désormais la totalité de la planète.

\section{L'œuvre}

J'ai envie de commencer par répondre en posant moi-même des questions :

- Par quels phénomènes êtes-vous conscient de votre existence, en tant que personne humaine, et comment expliquez-vous le fait que cette conscience soit limitée à votre seul esprit, à votre seul corps, à cette seule époque?

- Comment expliquez-vous notre perception et notre conception du temps comme phénomène irréversible?

- Dieu existe-t-il ? Pouvez-vous expliquer votre scepticisme, ou votre croyance?

- D'où vient l'Univers; où va-t-il ?

- Y avait-il quelque chose avant le Big Bang?

- Par quel processus naît la vie, "depuis l'acte initial jusqu'à la réalisation finale”?

Je pourrais aussi répondre par la poésie. René Char: "Comment me vint l'écriture? Comme un duvet d'oiseau sur ma vitre en hiver. Aussitôt s'éleva dans l'âtre une bataille de tisons qui n'a pas, encore à présent, pris fin" (La bibliothèque est en feu).

Le seul fait d'interroger, en parlant "d'idée initiale", de "processus suivi", et de "réalisation finale", me prouve que la question est posée par des personnes qui ont une certaine distance avec la conception et la réalisation "d'œuvres". On veut qu'il y ait planification quelque part, et on ne semble pas concevoir que tout cela fonctionne dans un équilibre précaire entre rationalisme constructiviste et pur hasard. Seuls les enfants de l'Université française peuvent poser les questions de cette manière.

Le hasard, l'irrationnel, le toujours imprévu, est une liberté énorme, fondamentale, et qui dérange. La liberté totale du projet créateur est esentielle, et sa permanente possibilité de remise en cause sur lui-même, à tout moment, est la condition abolue pour l'exercice de cette liberté. Nombre de commanditaires, d'organisateurs, de responsables politiques, etc., ne peuvent jamais véritablement concevoir et supporter cet aspect, pourtant déterminant, des choses. C'est pourquoi j'ai connu les moments les plus efficaces, les plus audacieux, et les plus heureux de mon travail, chaque fois que j'ai été mon propre producteur ; avec des entraves parfois très lourdes sur le matériel, 
les conditions de vie, la pratique, etc., mais aucune entrave au projet, à sa fluctuation, à ses renversements imprévus, à son cheminement infini.

Dans son ouvrage sur les Types psychologiques, C. G Jung fait de la "fantaisie créatrice" le lieu de réconciliation entre les puissances du conscient et celles, bien plus vastes, de l'inconscient: synthèses et luttes par lesquelles il tente d'éclairer toute l'histoire des hommes; conception à laquelle je me rallie.

30 Face à une société de plus en plus planificatrice, qui étouffe les conciences et ne crée plus aucun appel puissant : la liberté de l'art est le dernier refuge. Cette liberté, qui est la force de L'IMPREVISIBLE (faire qu'à un moment se révèle l'inattendu), sera toujours, par sa nature profonde, provocatrice et "révolutionnaire"! 


\section{Anthony Braxton}

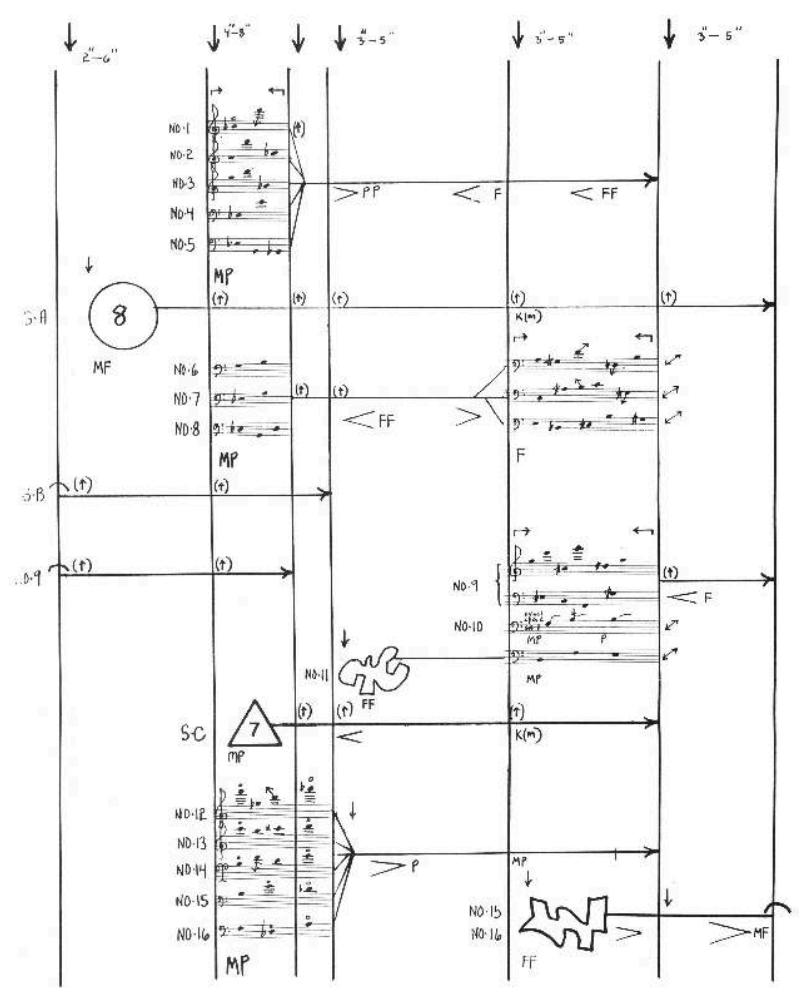

\section{Le compositeur}

1 Je considère mon œuvre (le travail de toute ma vie) comme une réponse à la restructuration de la musique $\mathrm{du} \mathrm{XX}^{\mathrm{e}}$ siècle, en particulier par les maîtres de la période post-seconde guerre mondiale (et tout spécialement la tradition Nouvelle-Orléans et l'époque moderne qu'illustrent des musiciens tels que Charlie Parker, John Coltrane et Omette Coleman, pour ne citer qu'eux) tout comme à la tradition de la grande musique (qui, à mes yeux, s'illustre particulièrement par Arnold Schoenberg, Karlheinz Stockhausen et John Cage). J'ai tenté de créer une entité phonique en trois parties 
perpétuelles qui démontre la beauté du chiffre 3 et fasse écho à la pensée du grand pharaon égyptien, Akhenaton. La réalité de mes recherches c'est d'établir :

1. une réalité architecturale ;

2. une réalité philosophique ;

3. un contexte synthétique.

2 La restructuration musicale des années soixante m'a permis d'ouvrir les portes à de nouvelles fusions, à des "équilibres nouveaux". Je considère mon travail comme lié à une "recertification... à la recertification du monde", comme à la profonde beauté de la transformation... la transformation du monde.

3 J'ai de la chance dans la mesure où, dès que j'ai pris la décision de consacrer ma vie à la musique, mon évolution a été conforme au modèle que je m'étais fixé à la fin des années soixante. J'ai pensé à la création d'une musique et d'une façon d'être sonore qui respectent l'évolution universelle de la musique - le tout fondé sur mon propre système de valeurs (spirituelles, s'entend). J'espère pouvoir continuer mon œuvre aussi longtemps que je vivrai sur cette planète. Cela me semble être la seule attitude compatible avec mon état d'“étudiant professionnel en musique".

\section{Le sérialisme}

4 Je n'éprouve qu'amour et respect pour l'évolution de la science musicale mondiale. Cela reste vrai même si chacun, bien sûr, doit poursuivre sa propre voie. J'aime les "polarités", mais il faut pourtant comprendre que l'évolution de l'art musical occidental a sacrifié la multiplicité de ses plates-formes d'information. C'est en supprimant les œuvres de nos grands maîtres féminins, en particulier celles de la grande Hildegarde Von Bingen, que nous avons hérité d'une plate-forme d'information déséquilibrée. Je ne cherche pas ici à trancher la question de la validité du sérialisme, mais plutôt à poser un dilemme existentiel : l'art musical occidental est lié au déclin de la spiritualité comme facteur unifiant du processus créatif. L'amour de l'improvisation et de la "présence individuelle" nous a menés à un point où nous devons remettre en question le strict processus qui nous est soumis au sein du complexe germanique d'information (ou d'affinités) et du système de valeurs qui y correspond. Maintenant que nous sommes entrés dans l'âge de l'ordinateur, je crois que l'on peut de plus en plus transférer la dimension mathématique pure aux systèmes robotisés et ainsi libérer l'interprète. Nous devons libérer les interprètes! En réalité, la question du sérialisme est étroitement apparentée aux problèmes originaux qu'ont fait surgir les technocrates européens aux dépens de la mystique européenne.

\section{Le concept de musique pure}

5 Je conçois la musique comme l'un des principes fondamentaux dans l'existence d'un univers de géométrie plane. Pour moi, la musique est tout - la seule question c'est de savoir ce que chacun désire vraiment savoir (sans oublier la position qu'on occupe dans le cercle). 


\section{Fonction de la musique}

6 J'ai tenté de fonder le travail de ma vie sur l'étude de la façon dont les Anciens considéraient la discipline musicale. Les Anciens ne concevaient pas toujours la musique comme coupée de son propre "soi" spirituel, vibratoire, mais plutôt comme une appréciation de l'équilibre des forces. Quand j'utilise l'expression d'"esthétique composite", je me réfère au même phénomène. Je crois que la musique et l'évolution de la science musicale sont d'une importance essentielle à la santé d'une culture. La musique est beaucoup plus profonde que nous ne le pensons en général, et la pollution sonore qu'engendre la communauté américaine du business a des implications très profondes aussi. Même aujourd'hui où l'ère reaganienne est balayée par le vent, nous sommes en mesure de constater une détérioration intellectuelle qui affecte l'ensemble du pays. Le dilemme que posent "les forces du marché", c'est que nous avons perdu la capacité d'entrer dans "sa" réalité. Le défi de la musique créative aujourd'hui est, selon moi, de redresser ce déséquilibre afin que la musique devienne l'instrument du peuple et non le contraire.

\section{Technique et pensée musicale}

7 J'ai le sentiment que les implications dynamiques issues de la restructuration musicale des années soixante exigent une nouvelle plate-forme d'observation qui interpelle de façon neuve la science, les valeurs émotionnelles, les valeurs spirituelles et surtout le système de valeurs tripartite (les décisions qui affectent l'individu).

8 J'ai dû, dans mon propre travail, construire un contexte identitaire. Je crois que l'on doit à présent réorienter la science du début du siècle afin qu'elle tienne compte du spectre vibratoire de l'humanité. Jouir pleinement de la grande musique de Louis Armstrong ou d'Edith Piaf, c'est comprendre la beauté d'une base réelle multivibratoire. Je crois que le système éducatif et les conservatoires devront complètement se remodeler dans les trente ans qui viennent. Nous devons trouver le moyen de communiquer ce qu'est "l'espace sonore poétique". L'existence de "l'action sonore" est le phénomène le plus important de la planète.

\section{Musique contemporaine et institutions}

9 Il faut absolument défier la réalité politique de nos institutions afin que lesdites institutions puissent s'ouvrir et servir le public. Nous sommes arrivés à une période où, même si nous pensons que les hommes blancs sont des gens magnifiques, il n'y en a pas moins d'autres êtres humains sur la planète ; aussi, peut-être serait-il juste de laisser quelques chances aux femmes-compositeurs, aux compositeurs africains ou même, Grands Dieux!, aux pauvres! Je dis que si les compagnies lyriques n'ouvrent pas leurs portes, il nous faudra prendre ces Bastille(s) comme en 1789 !

\section{Musique d'aujourd'hui et traditions}

10 De toute façon, je n'ai jamais accepté les informations ou les documentations européennes ou trans-européennes ; aussi suis-je tenté de penser que cette question ne 
me concerne pas personnellement. Une partie du problème réside dans l'éducation musicale mais également dans la musique comme partie d'une dynamique politique et sociale (des concepts tels qu'art majeur et art mineur,... l'IRCAM!, le jaaaazzz). Le monde entier attend que les habitants du monde occidental découvrent ces simples faits de base. Ou bien on se soucie de l'avenir, ou bien on laisse tout tomber!

\section{L'œuvre}

11 A ce point de ma vie - juillet 1989 - j'ai composé plus de 350 œuvres, longues et courtes, et, dans ce contexte, vous comprendrez que je ne puisse pas parler d'un seul style de composition musicale. J'essaie d'encourager mes élèves à refuser la peur et à s'amuser ! Oui, à s'amuser avec l'acte de création. Il n'y a pas de voie unique, et chaque personne doit s'interroger pour mieux comprendre ses propres polarités.

12 Mon problème n'a jamais été de savoir quelle approche était nécessaire à la composition, mais plutôt de savoir comment j'allais pouvoir emprunter dix dollars pour déjeuner. 


\section{Steve Reich}

Traduction : Serge Grunberg

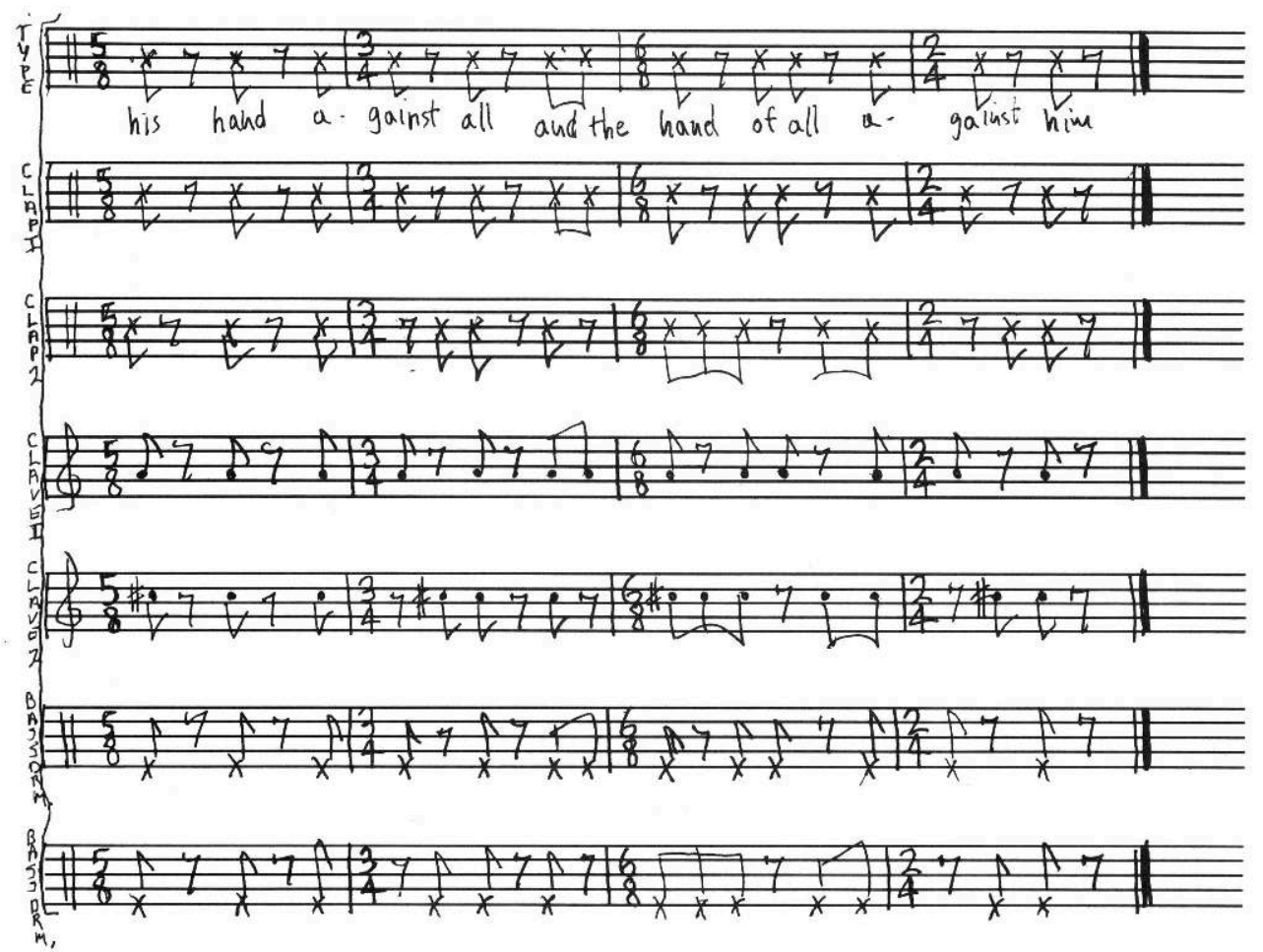

\section{Le compositeur}

1 En tant que compositeur, j'ai récemment été amené à m'intéresser de nouveau au discours comme source de la musique. Dans les années 1965-1966, avec It's Gonna Rain et Come Out, le discours était le composant unique. C'était une phrase courte qui, répétée et se déphasant graduellement, engendrait la totalité du morceau. En 1988, le discours a refait son apparition, mais cette fois comme source engendrant de la musique instrumentale. Ainsi, dans Different Trains (1988), c'est pour son contenu mélodique que chaque voix enregistrée a été sélectionnée, puis retranscrite en notation musicale; enfin, dans le morceau sous sa forme définitive, chaque fois qu'une femme parle, c'est 
un alto qui la double et chaque fois qu'un homme parle, il est doublé par un violoncelle. La totalité du matériau musical vient des mélodies discursives utilisées. Pour 1'avenir, je me suis mis à travailler à une musique pour la scène avec des réalisations vidéo où l'on pourra à la fois voir et entendre les sources documentaires de la musique.

\section{Le sérialisme}

2 Il est possible que personne ne distingue l'influence de la "pensée sérielle" sur mes premières œuvres telles que Piano Phase (1967). Je n'y utilise pas de technique sérielle à proprement parler, mais le degré d'organisation (quoique totalement différent) peut faire songer à "l'organisation totale" de la musique sérielle. Je sais qu'on considère mes premières œuvres comme absolument opposées à l'absence générale de rythme et de centre tonal qui caractérise la musique sérielle; c'est probablement vrai. Pourtant, il m'arrive de penser que la musique sérielle et Cage m'ont influencé musicalement, d'abord en me montrant la légitimité de n'importe quelle forme d'organisation radicale, et aussi en me rejetant du côté de mes propres inclinaisons rythmiques et tonales qui ne se satisfaisaient d'aucune de ces musiques. Cage et le sérialisme ont surtout représenté pour moi une influence à combattre.

\section{Le concept de musique pure}

Dans les années 1965-1966, j'ai composé It's Gonna Rain et Come Out, qui étaient des œuvres enregistrées n'ayant comme source qu'une voix parlée. De 1967 à 1982, je ne m'intéressais qu'à la musique purement instrumentale; je n'utilisais la voix chantée que comme une vocalise avec paroles pour imiter certains instruments dans un ensemble bien précis. Mais dès Tehillim, en 1982, puis avec The Desert Music, en 1984, j'ai commencé à vouloir offrir des textes aux chanteurs. Cela m'a permis de découvrir que, dès le moment où j'avais choisi le texte, j'étais forcé de faire, au plan musical, des choses que je n'aurais jamais faites autrement. Ce fut pour moi quelque chose d'extraordinairement stimulant. Un exemple : j'étais forcé de changer constamment les mesures afin de les faire coïncider avec les changements d'accents toniques des syllabes. Du point de vue harmonique, je me suis vu obligé d'utiliser davantage les accords chromatiquement altérés pour répondre de manière appropriée à certains problèmes textuels. En 1988, avec Different Trains, je me retrouvais aux prises avec une situation complètement nouvelle où se combinaient mon ancien intérêt pour le discours parlé comme matériau d'origine et l'écriture instrumentale que j'étais en train de développer - en l'occurrence, le quatuor à cordes. Le résultat me donna envie de consacrer désormais beaucoup de temps à l'exploration du matériel documentaire discours et sons divers - comme source génératrice de musique intrumentale/vocale.

\section{Fonction de la musique d'aujourd'hui}

4 Je ne peux que paraphraser la formule de J.S.B.: La fonction de la musique est de rafraîchir l'esprit et de stimuler l'intelligence. 


\section{Technique et pensée musicale}

5 Il n'est pas impossible que les musiques sérielle et dodécaphonique aient été des sortes de ruptures avec les principes naturels de résonance et de perception humaine de la musique. Ce qui pourrait expliquer pourquoi cette musique est à ce point restée impopulaire et pourquoi, après beaucoup plus de cinquante ans, le facteur ne siffle toujours pas du Schoenberg en faisant sa tournée. En ce qui me concerne, les principes naturels de résonance et de perception humaine de la musique ne constituent pas des limitations ; ce sont des données de la vie.

6 Quant à la technologie électronique, elle fait à présent partie de notre musique folklorique. Regardez la vitrine de n'importe quel magasin de musique et vous comprendrez ce que je veux dire. Pour ma part, il y a deux outils technologiques qui m'intéressent plus précisément : l'ordinateur et le sampler. Ce sont d'ailleurs tous deux des ordinateurs, mais le sampler est exclusivement destiné à réaliser des enregistrements digitaux. Je me sers de l'ordinateur pour sérier qualitativement des notations musicales pour partitions ou voix. Quant au sampler, je l'utilise pour enregistrer et intégrer des discours ou d'autres sons réels dans un morceau instrumental ou vocal. Il est évident que les nouveaux instruments donnent naissance à une musique nouvelle.

\section{Musique contemporaine et institutions}

7 De 1979, avec Variations, jusqu'à 1987, où j'ai terminé The Four Sections, je m'intéressais surtout à la musique orchestrale. Ce qui me passionnait, c'était d'écrire des partitions pour de multiples instruments à cordes et à vent, tout comme j'avais utilisé des percussions et claviers multiples (et même parfois des instruments à cordes et à vent) dans mes premières œuvres. Dans The Desert Music (1984), en particulier, j'ai pu créer mon propre orchestre, non seulement grâce à la composition, mais aussi en replaçant les cordes en trois groupes assis en arc-de-cercle autour d'un groupe central de percussion. Plus tard, j'ai appliqué ce type de conception orchestrale à The Four Sections afin que chacun des quatre mouvements fût centré sur les cordes seules, puis sur les percussions, sur les instruments à vent, et, finalement, sur l'orchestre tout entier. Immédiatement après avoir achevé The Four Sections, j'ai clairement ressenti que je n'écrirais plus de partitions pour orchestre symphonique dans un futur envisageable. J'ai finalement compris que la plupart des clichés qui courent sur les orchestres étaient vrais : comme si on s'arrangeait pour jouer la musique de Haydn à travers Schoenberg, et à vrai dire, à la base, cela revenait bien à ne pas refléter l'importance nouvelle des microphones, de la musique non occidentale, du jazz, du rock, des ordinateurs, des instruments électriques, etc. Ce qui revient à dire que, fondamentalement, l'orchestre ne reflète que l'Europe des XVIII et XIX ${ }^{e}$ siècles. Mais nous sommes en 1989 et je vis en Amérique.

8 Il y a aussi la "sociologie" de l'orchestre qui, sans aucun doute, est fortement liée à la musique qu'il joue; ce qui signifie également que de 20 à $80 \%$ des musiciens préféreraient ne pas avoir à jouer ma musique ou celle de mes contemporains. En revanche, l'Ensemble Intercontemporain en France, le Schoenberg Ensemble en Hollande, l'Ensemble Modem en Allemagne, le London Sinfonietta en Angleterre et le Group 180 en Hongrie forment, parmi d'autres, une nouvelle génération de musiciens 
dont le répertoire commence avec Schoenberg, Stravinsky et Bartók. Ces musiciens peuvent donner une interprétation très précise de mes œuvres ou de celles de mes contemporains parce que : 1 . Ils ne sont généralement pas plus de quinze à trente, et chaque instrumentiste prend la responsabilité unique de sa partie dans la mesure où il n'a généralement pas de remplaçant. 2. Ils sont parfaitement à leur aise avec l'électronique. 3. Ils ont une parfaite connaissance de ce type de musique. C'est avec cette génération de musiciens à travers le monde, dont mon ensemble fait partie intégrante, que je peux parier sur le futur.

\section{Musique d'aujourd'hui et traditions}

Mes relations avec la musique classique occidentale n'ont pratiquement rien à voir avec la tradition musicale qui va de Haydn à Wagner. Les seules influences que je pourrais citer incluent Debussy, Satie, Ravel, Stravinsky, Bartók, et Weill, pour le XX siècle, ainsi que Perotin et beaucoup d'autres sources d'avant 1750. Grâce à l'impressionnisme français, j'ai inconsciemment commencé à travailler avec des harmonies où la basse était colorée tandis que le registre moyen était structurel. Je dis "inconsciemment" parce que tous les Américains de ma génération ont été littéralement submergés de musiques influencées par l'impressionnisme, surtout celle des films, la musique populaire, Gershwin, le jazz, etc. Stravinsky et Bartók ont tous deux subi l'influence de Debussy, comme on l'a fait remarquer bien souvent. C'est un peu Debussy, beaucoup plus Bartók, et surtout le jazz, qui m'ont éveillé à la musique non occidentale ; pour ce qui me concerne, ce fut bien vite plus un intérêt pour les structures non occidentales que pour les sons. C'est de Weill que j'ai d'abord reçu la confirmation qu'il faut prendre au sérieux les sources populaires; il m'a également appris que, lorsqu'on écrit de la musique lyrique, on doit choisir un ensemble et un style vocal appropriés à son temps, à son lieu et à son livret, et non tenir pour établi qu'on est forcé d'avoir un orchestre et des voix de bel canto. L'influence de Perotin fut surtout de me faire comprendre que l'on pouvait mener l'augmentation vers des extrêmes et ne pas se cantonner à des multiples de deux. Enfin j'ai appris des traditions médiévale, renaissance et baroque (autant que de Bartók et Webern) que le canon est un puissant outil de composition qu'on peut appliquer à n'importe quel son.

Quant aux influences de jeunesse, en dehors de la musique classique occidentale, il y a eu le jazz, et surtout le be-bop de Miles Davis et Kenny Clarke, ainsi que le jazz prétendument "modal" de John Coltrane. Dans les années soixante et au début des années soixante-dix, ce furent plutôt les tambours d'Afrique de l'Ouest, le Gamelan balinais et les chants hébraïques. De Miles Davis, j'ai appris que peu de notes pouvaient être plus efficaces que beaucoup; de Kenny Clarke, j'ai appris qu'un rythme léger, flottant plutôt simple, pouvait s'avérer plus efficace qu'un rythme trop riche et trop "plein". Coltrane m'a appris qu'on peut faire beaucoup dans la musique avec quelques changements d'harmonie. La musique d'Afrique de l'Ouest m'a enseigné que les motifs répétitifs superposés ou les temps forts ne coïncident pas, ne sont pas uniquement limités aux "boucles" sur magnétophone, mais aussi à d'autres traditions musicales. La musique balinaise m'a appris d'autres détails sur les motifs répétitifs synchronisés; sans oublier que, dans le Gamelan Gambang, on trouve des motifs beaucoup plus longs. Les chants hébraïques m'ont fait découvrir les sources historiques du chant grégorien ; sur un plan beaucoup plus technique, j'ai compris comment on pouvait arranger des 
motifs très courts pour qu'ils forment des lignes monophoniques beaucoup plus longues, le tout au service d'un texte sacré. Mais j'ai surtout appris de toutes ces sources non occidentales ce qu'était la tradition orale comme manière de transmettre la musique sans notation musicale, ce qui eut la vertu de clarifier le besoin que j'éprouvais de travailler avec mon propre ensemble (ou avec des ensembles similaires) où les gens n'avaient pas seulement une bonne formation musicale, mais aussi les connaissances, l'équipement et les dispositions favorables à la musique que je fais.

\section{L'œuvre}

11 J'ai toujours commencé à écrire mes œuvres de deux façons : ou je pense d'abord à la forme, ou j'entends d'abord le contenu. Dans des morceaux aussi différents que Four Organs (1970) et Different Trains (1988), j'ai d'abord pensé à la forme et/ou aux techniques de composition, sans avoir la moindre idée sur ce que cela "donnerait". Pour Four Organs, j'ai pensé : "les accords courts deviennent longs par augmentation". Pour Different Trains, j'ai pensé : "le discours et les autres sons mixés au sampler produisant des lignes mélodiques pour instruments à cordes". Je n'avais pas la moindre idée de la façon dont la musique allait "sonner" pour aucune de ces deux pièces avant de me mettre au travail. A l'inverse, c'est en rêve que j'ai entendu le motif de base d'une de mes premières œuvres pour bande magnétique, Melodica (1966); dans bien d'autres morceaux, à commencer par Music for 16 Musicians, tout a pris forme autour d'un cycle d'accords qui constituaient la base de la composition.

12 Il y a eu une grande diversité dans les moyens de composition. La plupart de mes pièces ont été écrites au piano ou au clavier électrique. Je me sers habituellement d'une bande magnétique à plusieurs pistes pour entendre et tester les détails contrapuntiques. Récemment j'ai utilisé une guitare "bourrée" de claviers samplerisés pour écrire Electric Counterpoint ; en effet, un piano aurait donné une impression complètement fausse du son que j'obtiendrais à la guitare, et surtout avec plusieurs guitares superposées. Dans Different Trains, j'ai utilisé en même temps le sampler (cette fois-ci "bourré" de discours et de bruits de train) et le sequencer de mon ordinateur pour préparer les premières pistes de ma bande multi-pistes en studio, avant que le Kronos Quartet ne vienne y ajouter ses enregistrements de quatre instruments à cordes superposés.

13 Le stade final, pour toutes les pièces, est le moment de répétition, durant lequel on peut effectuer de légères altérations dans les partitions instrumentales afin d'en faciliter l'exécution. Un compositeur qui n'accepterait pas les suggestions de bons interprètes pour améliorer une partition serait vraiment bête.

14 Ainsi mon approche est-elle avant tout pratique : je commence avec la conception originelle - forme et contenu - et j'utilise ensuite tous les outils nécessaires à l'achèvement de l'œuvre. 


\section{Sylvano Bussotti}

Traduction : Vivianna Aliberti

\section{Le compositeur}

1 Un développement lent et graduel - "normal", voudrions-nous pouvoir dire. Il y a eu sans aucun doute des découvertes et des influences; des circonstances extérieures très importantes, exception faite des derniers mois de la dernière grande guerre - 1945 qui, me contraignant à interrompre mes études au Conservatoire de Florence, $\mathrm{m}$ en libérèrent définitivement. L'avenir suivra graduellement ; lentement, espérons-le, par le même chemin.

\section{Le sérialisme}

2 Nous savons aujourd'hui comment le sérialisme a rompu avec le passé de façon bien moins radicale qu'il n'y pouvait paraitre. Sa force technique, conceptuelle, voire peutêtre aussi sa force extatique, proviennent justement de ce fait spécifique. Si, aujourd'hui, un travail de réflexion théorique approfondi semble faire défaut, il s'agit d'une faiblesse générale, qui ne concerne pas seulement l'expérience sérielle.

\section{Le concept de musique pure}

3 La musique ne peut certes se targuer d'aucun privilège par rapport aux autres disciplines artistiques. Dans les arts, la contamination est un fait établi - mieux, une nécessité vieille presque d'un siècle. Tant pis pour ces musiciens aveugles et illettrés qui, inexorablement, sont condamnés à finir sourds. Pour le reste, la question ne fait que rebondir depuis des temps bien plus anciens jusqu'à nous, et elle est destinée à se répercuter à perpétuité dans le futur. Se référer à tel ou tel modèle qui nous est contemporain n'a donc guère de sens : ce qui paraît bien plutôt nécessaire, c'est la connaissance la plus large possible - et mise à jour - des artistes et des diciplines de tout temps et de tout lieu. 


\section{Fonction de la musique d'aujourd'hui}

4 Question oiseuse, trompeuse ; liquidée depuis longtemps avec fermeté et autorité, et pourtant toujours prête à se représenter avec pétulance et une bonne dose d'hypocrisie culturelle. Sans parler du soi-disant rôle social de l'apprentissage musical. Dans la société d'aujourd'hui comme dans toute société, la musique doit être enseignée et apprise.

\section{Technique et pensée musicale}

Ce n'est certainement pas la technologie qui imposera des changements radicaux de la pensée et de la pratique musicales, quel que soit son degré de définition ou de délire. L'humaine faculté d'invention ne dira jamais que les seuls mots capables de sens et d'accomplissement nécessaire. L'évolution des systèmes musicaux a toutes les chances de s'avérer beaucoup plus riche que ne le tolère l'ignorance générale. Et cela vaut également pour toute matière, et pour toutes les formes possibles de communication. Ce qui demeure indispensable, ce n'est pas tant l'ordinateur ou toute autre petite "machine célibataire", mais l'originalité de la pensée même, non dissociée du travail manuel ; exercice obstiné et impondérable.

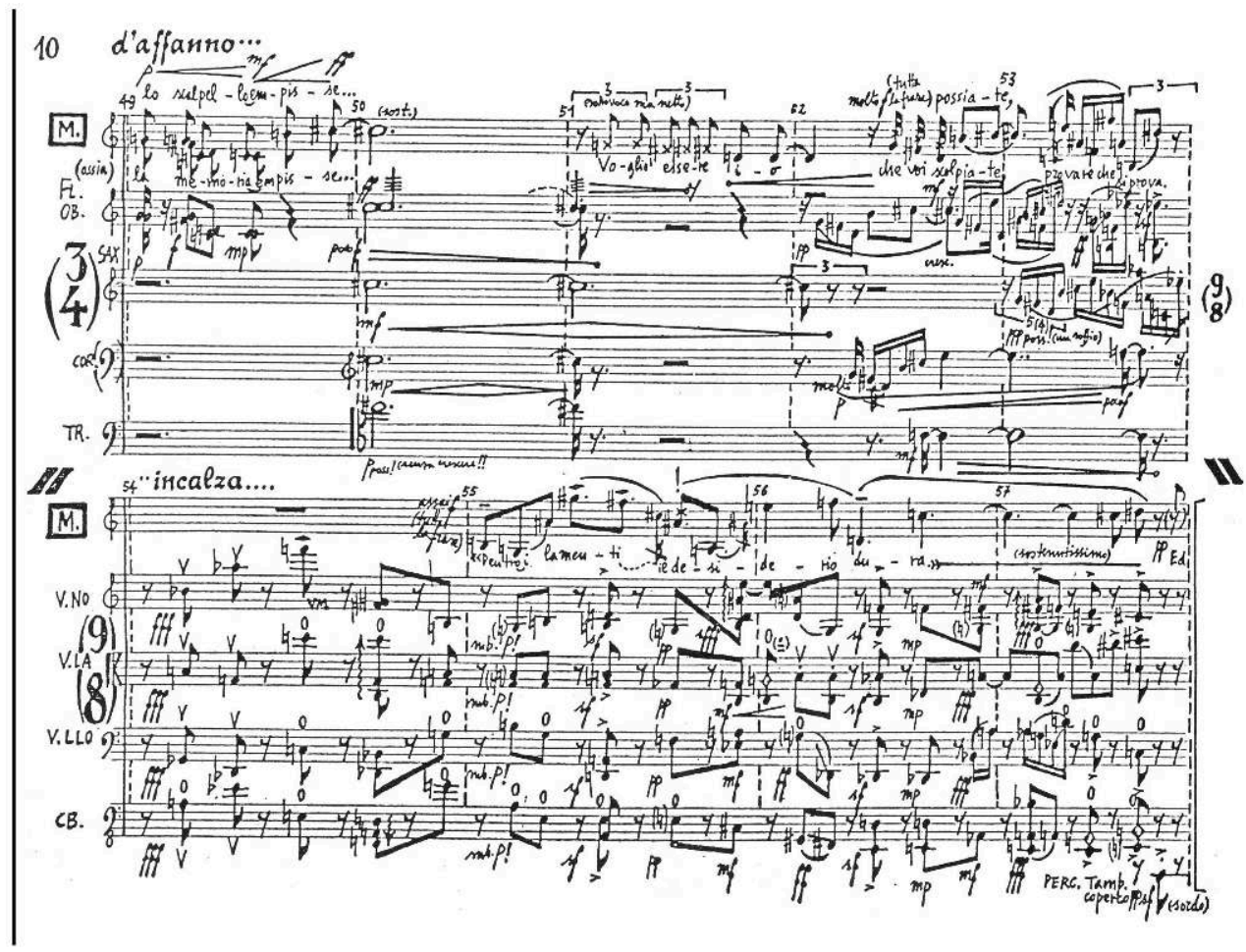

\section{Musique contemporaine et institution}

Bien sûr, l'organisation de la vie musicale en vogue à l'heure actuelle paraît bien éloignée de la musique vivante. Il ne sont pas beaucoup, ceux qui ont la force nécessaire et le pouvoir de décision permettant de créer un contraste radical, comme il faudrait, 
par rapport à cette situation ; le défaut le plus voyant - souvent le plus scandaleux - se manifeste avec évidence au niveau de l'école.

\section{Musique d'aujourd'hui et traditions}

7 On a toujours parlé de rupture entre les traditions et les musiques actuelles là ou c'est précisément le contraire qui est vrai. Les idiomes dit populaires, présents dans le monde sous leurs différents avatars dans des civilisations même très éloignées les unes des autres, concernent le langage musical dans son ensemble. L'incidence de tel ou tel trait idiomatique sur le style d'un compositeur, ou d'une de ses œuvres spécifique, peut avoir des conséquences infinies, propres à déboulonner la partialité de toute théorisation exclusive.

\section{L'œuvre}

8 Une telle question ne devrait pas figurer dans un questionnaire (... questionnaire dont on devrait d'ailleurs franchement critiquer l'idée même!). Pareillement, il n'est dit nulle part qu'il doive forcément y avoir des "idées de départ" ou que l'on doive parvenir à leur "réalisation finale". Nous supposons des parcours moins puérils et bien plus accidentés. 


\section{Toru Takemitsu}

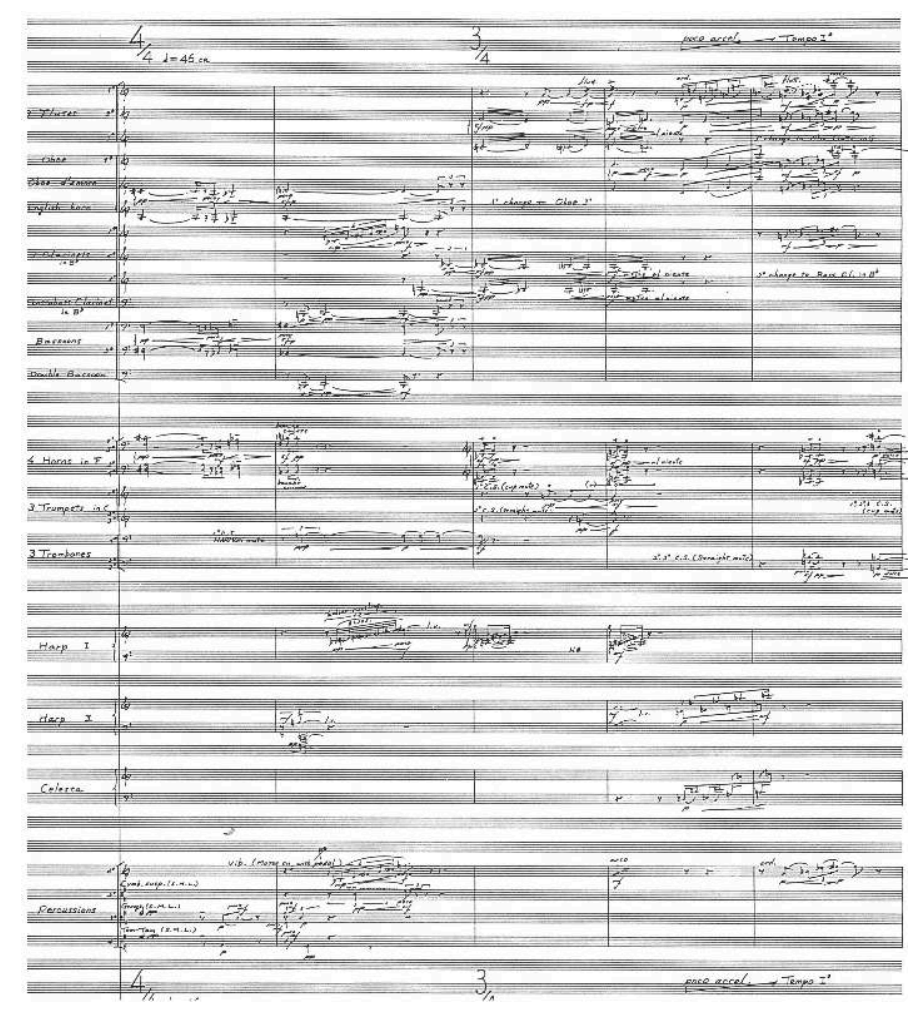

\section{Le compositeur}

1 Je reconnais avoir fait des progrès du fait de mon expérience acquise en technique de composition, mais ils n'ont cependant pas grand-chose à voir avec l'essence même de l'acte de composition. Mon moi intérieur reçoit tout naturellement un grand nombre d'influences venant des multiples changements de situation de ce monde, et l'influence que je peux subir de la part des diverses techniques musicales représente bien peu de choses par rapport à ce que je peux recevoir de cette nature qui m'environne. 


\section{Le sérialisme}

2 La technique sérielle, sur le plan de la pensée de la structure musicale, est une invention importante, mais, en même temps, elle a tendance à n'être accomplie en tant qu'événement que sur la surface plane représentée sur le papier. Le son y est pris comme une simple fonction et risque de se retrouver emprisonné dans une esthétique stérile. On peut utiliser la technique sérielle ou tout autre système de même nature, mais l'important est d'avoir toujours à l'esprit les lois naturelles de la physique du son.

\section{Le concept de musique pure}

3 En dehors de la musique, je suis toujours influencé par toutes les autres formes d'expression artistique. Les beaux-arts et le cinéma, en particulier, exercent sur moi une grande influence.

\section{Fonction de la musique}

4 En tant que musicien, je compose avant tout pour moi-même, mais en espérant que cela réveillera, en ceux qui écouteront ma musique, le sentiment de la nature caché à l'intérieur de chacun d'entre nous. Moi, je compose ma musique comme un témoignage de l'amour que je porte à l'existence humaine, en tant que partie du grand tout de la nature, indépendamment des pays et des peuples.

\section{Technique et pensée musicale}

5 Je crois que la musique contemporaine a tendance à devenir bien trop intellectuelle. Beaucoup de choses ont perdu toute sensualité musicale. Je pense cependant que nous ne devons pas être craintifs vis-à-vis de l'utilisation de la technologie (les ordinateurs y compris). Pour un artiste, il n'y a rien de plus dangereux que la stagnation de soimême. Nous nous servons de la technologie pour amplifier notre imagination (auditive), et si cela nous permet de voir se créer un nouveau monde musical, les compositeurs devront l'affronter modestement, mais aussi positivement.

\section{Musique contemporaine et institutions}

6 On ne peut pas généraliser à l'heure actuelle, car je crois que les problèmes sont différents selon les systèmes de chaque état, mais le milieu social où se place la musique contemporaine n'est pas toujours favorable. Je crois que le système, y compris pour l'éducation, est encore trop conservateur, et même, trop fermé.

\section{Musique d'aujourd'hui et traditions}

7 Etant un Japonais qui fait de la musique européenne, mon problème est différent de celui des compositeurs d'Europe et d'Amérique. Mais, de la même façon que j'aime ma propre tradition, je ressens un grand respect et un profond amour à l'égard de la 
tradition de la musique européenne. En apprenant la musique européenne, je veux relativiser la tradition de mon propre pays. Et, dans la musique que je compose, je veux exploiter l'essence de la musique (traditionnelle) japonaise et non l'utiliser en tant que matériau superficiel. C'est la recherche de la couleur, de la particularité de la forme, et de la structure temporelle.

\section{L'œuvre}

8 Mes œuvres prennent naissance par divers procédés de composition. Et ces procédés, justement, sont toujours pour moi le plus important. J'obtiens l'inspiration de la création par différentes influences extérieures qui produisent de l'effet sur mon moi intérieur. Mon procédé de composition diffère toujours selon ces influences. 


\section{Luciano Berio}

1 Trop de questions, hélas : il faut bien garder un peu de mystère ! La création musicale, comme toute forme de créativité, pour devenir une valeur permanente et même révolutionnaire, doit être contrôlée par la raison, et, en même temps, elle doit rencontrer des obstacles.

2 Enfin, puisque j'ai évoqué le mystère, peut-être qu'une réponse zen est ici convenable : "Tout le monde connaît le bruit de deux mains frappées, mais quel est celui d'une seule?" 


\section{Marcel Landowski}

\section{Le compositeur}

1 Les découvertes électroacoustiques ont donné des possibilités extraordinaires d'élargissement de la palette sonore, notamment avec des instruments tels que les synthétiseurs ou certains instruments traditionnels électriques.

\section{Le sérialisme}

2 Je pense que toute loi inventée, et non découverte, ne vit que le temps d'une mode.

3 Toute idée de rupture, de table rase, est génératrice de mort.

\section{Le concept de musique pure}

Comme je suis passionné de théâtre, je crois à la musique pouvant sublimer le verbe.

\section{Fonction de la musique d'aujourd'hui}

5 La musique d'aujourd'hui, comme celle de demain, se doit d'être un chant d'espérance donc de spiritualité, même dans les œuvres les plus sombres.

\section{Technique et pensée musicale}

6 Oui, dans certains cas, elle me paraît être une rupture. 


\section{Musique contemporaine et institutions}

\section{Musique d'aujourd'hui et traditions}

7 Je crois que la musique d'aujourd'hui doit être proposée tout naturellement à tous les publics aussi bien dans les formes traditionnelles que novatrices.

\section{L'œuvre}

8 Comme nous sommes nous-mêmes mystère, l'œuvre naît dans le mystère, puis s'ordonne à partir de notre intelligibilité des choses en s'appuyant et en tirant sa sève du monde de la sensibilité. 


\section{Henri Dutilleux}

\section{Les influences}

1 Les polyphonistes de la Renaissance, les deux Ecoles de Vienne (Beethoven pour le renouvellement des formes, l'Ecole de Schoenberg, mais assez tardivement), les musiciens d'Europe centrale (Bartók, etc.), le patrimoine français - les grands créateurs (Berlioz, Debussy) mais aussi Ravel (pour la mise en œuvre), Roussel, à la fin de mes études, mais passagèrement et comme "antidote" contre une tendance à la verticalité.

2 Les influences et découvertes littéraires aussi : Shakespeare, Baudelaire (tout autant pour ses essais critiques sur l'art ou des pages comme Fusées, que pour son univers poétique, Proust, Gide (pour le Journal), Julien Gracq (Les “Lettrines"), Yves Bonnefoy (tout récemment).

3 Dans les arts plastiques, surtout la peinture (Van Gogh, Gauguin, Nicolas de Staël, Kandinsky,...).

4 Les cinéastes : Bunuel, Fellini...

\section{Le sérialisme}

5 Le placer dans une perspective historique (comme une étape, plus que comme une rupture) à une époque où le besoin de rigueur succédant à une période d"“abandon", de laisser-aller, notamment en France (esprit "Groupe des Six"), à un certain esprit "french fashion", particulièrement dans l'entre-deux-guerres. Si j'ai refusé l'aspect dogmatique, j'ai apprécié le souci d'organisation du langage, même si l'entreprise conduisait à étendre, par la suite, le système sériel aux différents paramètres (après les hauteurs, les durées, les timbres, l'intensité) ce qui me semblait une vue de l'esprit.

6 N'ayant pas été un musicien sériel, je me suis retrouvé quelque peu isolé, et cette solitude relative (car mes partitions, dans les années cinquante et soixante, commençaient à circuler dans le monde) m'a conduit à me remettre en question. Je n'aurais sans doute pas été le même si je n'avais pas traversé cette période, une période 
assez inconfortable pour tout le monde, pour les sériels eux-mêmes mais avec un peu de retard.

7 Rupture avec le passé ? Délibérée ou non, la fracture ne s'est pas réellement produite. Il reste les œuvres. Stravinsky, dans sa période sérielle, a écrit Agon et c'est encore du Stravinsky.

\section{Le concept de musique pure}

8 Il n'y a pas de règle. On peut écouter le Prélude à l'après-midi d'un faune sans penser à Mallarmé. A l'extrême, il n'est pas beaucoup plus difficile, de nos jours, de se dégager du programme autobiographique de la Fantastique. Au-delà de son titre, le symbolisme du "Jardin du sommeil d'amour" chez le Messiaen de la Turangalila, ne nous écarte pas de la pure musique, sublime en cet épisode.

9 Comme il faut bien parler de soi-même, j'ai écrit “Tout un monde lointain" après m'être replongé dans l'univers de Baudelaire, de même que ma pièce orchestrale Timbres, Espace, Mouvement ou La nuit étoilée est le résultat d'un choc émotif: celui éprouvé devant la toile visionnaire de Van Gogh. Dans les deux cas, l'impulsion est donnée par des éléments extérieurs à la musique. Il s'agit d'un stimulant. Je souhaite parfois que l'auditeur oublie ces références, s'abstienne de lire les notices des programmes.

10 Mais là, il s'agit d'impulsions "au premier degré". Très souvent aussi, les sources de l'élan créateur peuvent être recherchées dans des expériences plus lointaines, en des domaines très divers: ainsi, voici quelque vingt années, la visite d'une exposition Tinguely, avec ses étranges machines, avait aiguisé en moi je ne sais quel besoin d'écrire. De même pour certains films (chez Losey, Bergman, Resnais) dont l'ingéniosité des structures, l'invention formelle, présentent des analogies avec la composition, d'où leur effet stimulant.

\section{Fonction de la musique d'aujourd'hui}

11 Question à poser aux sociologues, aux philosophes.

\section{Technique et pensée musicale}

Je me refuse à revenir sur l'éternel problème d'un prétendu viol des "principes naturels de la résonance", un cliché dont on a souvent usé dans les années cinquante pour condamner le dodécaphonisme.

D'ailleurs, pour moi, il n'y a pas une musique contemporaine, mais diverses formes d'expression de la pensée musicale de notre temps, avec toutes les facettes que présente tel ou tel créateur. Certaines démarches impliquent des recherches d'ordre collectif (par exemple tout ce qui se rattache à l'électro-acoustique ou à l'usage de l'ordinateur) et il me parait naturel que des musiciens de vingt ans n'y restent pas étrangers. D'autres formes d'expression exigeront toujours du compositeur une réflexion solitaire (le travail artisanal, à la table de travail). Les deux démarches peuvent être complémentaires et pour ma part, je ne souhaite pas une "mutation 
radicale de la pensée et de la pratique musicales". Je la redouterais, mais surtout je n'y crois pas.

\section{Musique contemporaine et institutions}

Dans le monde entier - avec quelques variations - on constate le même décalage entre la création contemporaine et les institutions traditionnelles ou centres de diffusion (à l'exception des organismes de radio). La part de musique de notre temps n'est pas plus considérable dans les programmes de la Philharmonie de Berlin, de celle de New York, des grands orchestres de Londres, etc., qu'elle ne l'est dans les programmes de l'Orchestre de Paris. Par contre, il est notable que les théatres lyriques, en Allemagne surtout, mais aussi aux USA, en Grande-Bretagne et dans certains pays Scandinaves s'ouvrent bien davantage qu'en France aux nouvelles créations. Les structures d'accueil sont d'ailleurs traditionnellement différentes.

Les Conservatoires? En France, par exemple, dans les deux "Conservatoires supérieurs" (Paris et Lyon), on n'observe heureusement pas ce même décalage au niveau de l'enseignement. De nos jours, les étudiants sont "en prise directe" avec la vie musicale la plus active, la plus provocante (liens avec les organismes de radio, avec l'Ensemble Intercontemporain, antennes à l'IRCAM). Dans les années précédant la guerre, lorsque je terminais mes études rue de Madrid, les relations avec le monde extérieur étaient, par contre, à peu près nulles (j'ai mis longtemps à me remettre de cette carence!). Pourtant, Stravinsky séjournait souvent à Paris (il s'est présenté à l'Institut en 1936 et il n'a eu que cinq voix au premier tour, plus aucune voix à partir du troisième !...), Bartok était lui-même passé plusieurs fois à Paris, d'autres encore, même Schoenberg avant de s'exiler aux Etats-Unis en 1933, mais nous n'en savions rien...

\section{Musique d'aujourd'hui et traditions}

Je n'ai guère envie de m'étendre sur l'idée de tradition et je redoute ce mot, comme tout ce qui s'y rattache par analogie: habitudes, culte du passé, académies, Institut, filiation, héritage, préjugés...

Les traditions doivent souvent être violées et celles qui peuvent le mieux nous nourrir, nous enrichir, émanent souvent de l'extérieur, de l'étranger. J'évoque souvent la pensée de Gide selon laquelle un art national, pour s'épanouir, a besoin du "levain de l'étranger". Peut-être trouvons-nous mieux ce "levain" auprès des civilisations lointaines, mais si je songe aux musiques extra-européennes, par exemple, ce qui m’a stimulé, c'est beaucoup plus le sentiment d'une perception particulière du temps que la découverte d'instruments inconnus chez nous, malgré toute la puissance magique dont ils sont porteurs.

Dans un autre domaine, le jazz à l'état pur (que Boris Vian m'a fait mieux connaître dans les années cinquante) ne m'a pas vraiment nourri mais je ne peux réentendre la voix de Sarah Vaughan sans être transporté. Certaines formations réduites du jazz actuel (par exemple, cuivres et percussion) ont un relief des plus séduisants. 


\section{L'œuvre}

19 Comment naît une œuvre ? Le mystère est total et le processus toujours différent, selon le matériau sonore utilisé.

20 Avoir ou non, en commençant un travail, la vision d'ensemble de l'œuvre à venir ? Belle perspective, mais heureusement les incidents de parcours peuvent sinon altérer l'idée initiale, du moins modifier sensiblement la forme et finalement l'enrichir.

21 Savoir renoncer à telle idée valable en soi, mais inopportune dans le contexte choisi. Noyau central: potentialité d'une formule particulière - rythmique, harmonique (accord pivot), linéaire (un certain mélisme), timbres - qui irradie l'ensemble.

22 Saisir l'instant.

23 Etre disponible, en "appétit de travail", condition des plus difficiles à réaliser, du fait des agressions quotidiennes : courrier, sollicitations constantes, les voyages eux-mêmes qui - s'ils vous permettent parfois de "rebondir" - brisent souvent la continuité ("Le travail, force progressive et accumulative portant intérêts comme le capital, dans les facultés comme dans les résultats" écrit Baudelaire dans Fusées).

$24 \quad$... "L'Art est un combat - il faut y mettre sa peau" (Van Gogh). 


\title{
Entretien avec Pierre Boulez
}

\author{
Philippe Albèra
}
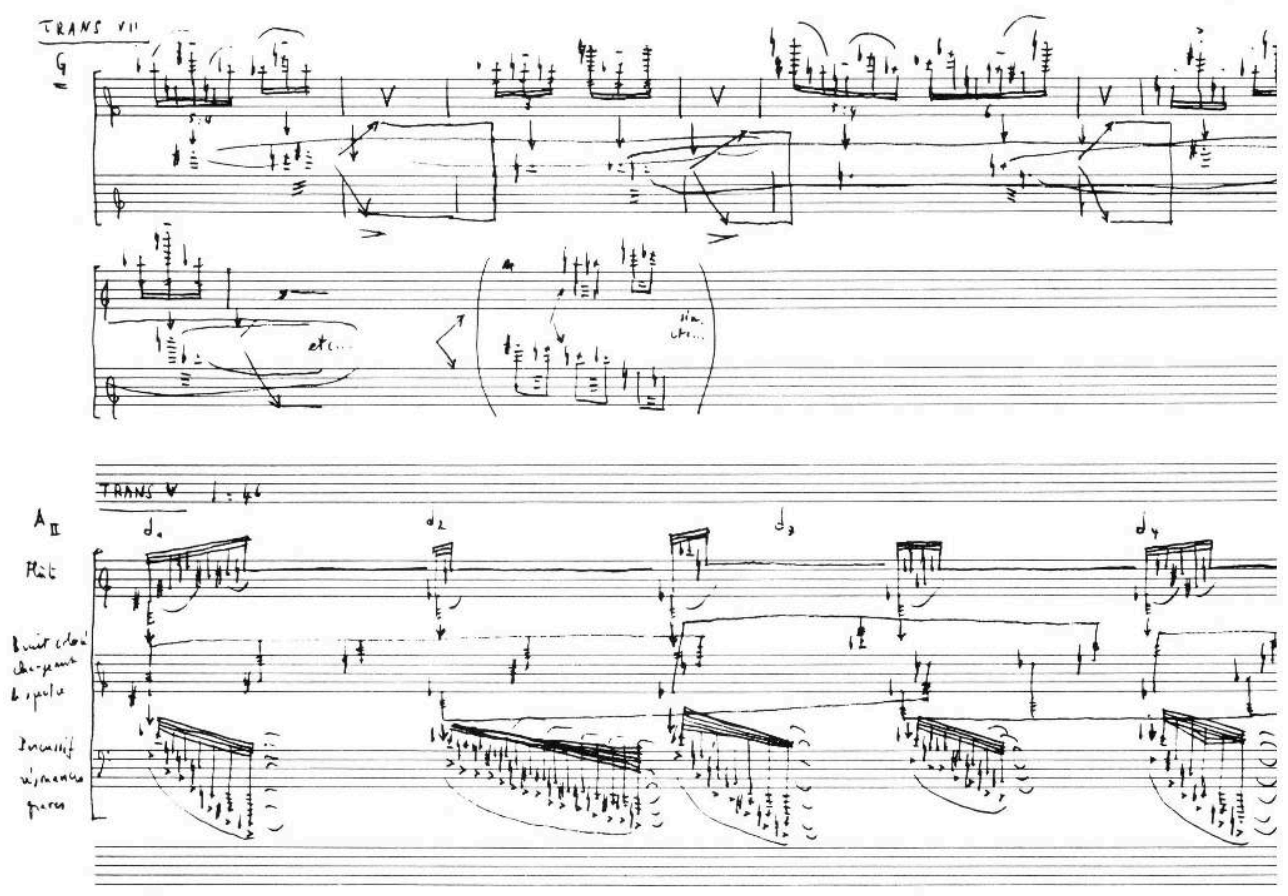

1 Vous avez été, depuis vos débuts, à la recherche d'une loi, et votre réflexion s'est beaucoup attachée à la notion de système. Mais si le sérialisme, dans les années cinquante, était partagé par un groupe de compositeurs, aujourd'hui, chacun a une démarche individuelle. N'est-il pas absurde qu'un système soit le fait d'un individu isolé?

Pierre Boulez: Il existe dans la musique d'aujourd'hui des éléments de base, même primitifs, qui sont les mêmes chez plusieurs compositeurs : les relations chromatiques, l'absence de périodicité rythmique traditionnelle, l'invention de formes qui ne sont plus basées sur des cadres préétablis. Il ne s'agit pas de règles, mais d'une attitude générale, d'ailleurs plus souvent faite de refus que d'acceptations. C'est à partir de cela que l'on fait des choix tout à fait personnels. 
3 - Qu'est-ce que les systèmes musicaux, aujourd'hui, légalisent? Quel contenu pourraiton leur donner?

4 Pierre Boulez : Il était déjà difficile, à la fin du dix-neuvième siècle, de lier les systèmes musicaux à une philosophie ou à une représentation du monde, comme on a pu le faire jusqu'au dix-huitième siècle. La divergence entre les mondes scientifique, philosophique et artistique s'est accentuée de plus en plus, et très peu de gens ont été capables, avec les connaissances suffisantes, d'en déduire des "systèmes". Les musiques chinoises ou indiennes, par exemple, sont vraiment rattachées à une explication du monde, à une mythologie. Mais le symbolisme et les métaphores de cette sorte ont complètement disparu de notre musique. Les progrès de la connaissance nous ont éloignés de certaines correspondances littérales. Aujourd'hui, il faut être naïf pour y revenir, ou inventer une métaphysique et une science ad-hoc.

5 Les concepts artistiques, toutefois, s'ils n'en dérivent pas directement, sont plus ou moins influencés par des conceptions scientifiques, philosophiques ou autres qui existent à l'état diffus; c'est le fameux Zeitgeist! Les énormes développements acoustiques, par exemple, qui ont eu lieu depuis le dix-neuvième siècle, nous empêchent de concevoir le phénomène acoustique aussi simplement qu'alors. Nous sommes aujourd'hui conscients de nombreux détails qui enlèvent toute validité aux systèmes acoustiques apparus dans le monde scientifique au dix-huitième siècle. Il est par exemple impensable, avec les analyses que nous faisons actuellement, de fonder un langage musical sur la théorie acoustique des premiers harmoniques.

6 - Au début des années cinquante, par exemple, aviez-vous conscience d'être dans la mouvance des sciences humaines de l'époque, et notamment du structuralisme?

7 Pierre Boulez : J'en étais proche, certainement.

8 - Influencé directement?

9 Pierre Boulez : En ce qui me concerne, je voyais cela de suffisamment près pour savoir que nous avancions dans la même direction.

10 - Dans votre essai intitulé "Le système et l'idée" ${ }^{1}$, vous dites qu'une forme qui ne revient pas sur elle-même rend impossible d'établir tout système général...

11 Pierre Boulez: Oui, je pourrais prendre la comparaison de l'architecture : la forme musicale était semblable autrefois à ces architectures classiques faites pour être vues sous un seul angle, dans une perspective précise. Les formes musicales, bien que le génie les ait transformées et modelées à son avantage, étaient des formes acceptées : on ne peut nier l'existence des premier et second thèmes, de l'exposition et du développement! Déjà Beethoven a forcé de telles notions, même s'il n'a pas été suivi par les romantiques-Mendelssohn, Schumann, Brahms, et même Berlioz - qui se sont contentés d'un monde prétabli. Les derniers quatuors de Beethoven ont envahi l'écriture de Wagner assez tardivement, passé les années soixante ; mais avec Wagner, on peut se demander si c'est le dramatisme de son théâtre qui a entraîné son concept formel ou si c'est la conception musicale qui l'a conduit au non-retour dans la construction dramatique. Ce que j'appelle non-retour, c'est l'absence de répétitions littérales: chez Wagner, les retours existent, mais toujours dans des contextes différents.

12 - Le concept de répétition, qui fut problématique dans les années cinquante, n'a-t-il pas été traité un peu grossièrement à l'époque - je veux dire que l'on a classé toute répétition dans la catégorie de la répétition littérale? 
13 Pierre Boulez : Oui, certainement ; et c'est là que tout le développement s'est fait. Nous sommes partis d'une position très radicale, souvent avec des œuvres très courtes d'ailleurs, qui nous a permis d'envisager la forme selon un autre point de vue. Il y a deux ans, au Collège de France, j'ai précisément fait mon cours sur l'enjeu thématique : comment s'est transformée la notion de thème jusqu'à nos jours? Il faut, en effet, qu'une telle notion existe, parce qu'on ne peut concevoir une musique sans thème. La musique peut être plus ou moins amorphe, le thème plus ou moins dilué ; j'ai appelé cela des coefficients thématiques : il peut y avoir une façon de concevoir qui irrigue une forme, qui permet d'obtenir une enveloppe particulière.

14 - Si l'on prenait des œuvres comme le Marteau sans maitre ou la Troisième sonate pour piano par exemple, quels en seraient les éléments thématiques?

15 Pierre Boulez : C'est très net : dans la Troisième sonate, ce sont les points et les blocs (blocs frappés ensemble, blocs analysés, etc.).

16 - N'est-ce pas plutôt une stratégie qu'un élément thématique?

17 Pierre Boulez : Non, ce sont les éléments thématiques qui vous forcent à découvrir des manières d'analyser les rapports de ces blocs : on les décrit d'une certaine façon. Dès lors, la notion de thème se greffe sur la notion de bloc. Une surface ou un volume peuvent être décrits, alors qu'un point ne peut pas être traité de différentes manières : il existe en tant que tel.

18 - A l'intérieur des points, il n'y a donc aucun repère...

19 Pierre Boulez: Non, c'est complètement indifférencié, alors que les blocs sont extrêmement différenciés. A part cela, il y a d'autres enveloppes plus générales, qui sont des stratégies : les points, par exemple, sont toujours écrits dans le registre médian, alors que les blocs vont de l'extrême aigu à l'extrême grave.

20 - Dans Rituel et Répons, vous êtes revenu à une notion thématique plus traditionnelle, à des figures - c'est le mot que vous utilisez d'ailleurs - que l'on peut identifier facilement... Le mot figure remplace-t-il celui de thème?

21 Pierre Boulez : La figure irrigue un passage. Dans une œuvre comme Répons, où il faut tenir compte de la durée, il y a des notions qui évoluent dans le temps. Par exemple, un élément thématique irrigue le tout: c'est une série de doubles croches régulières entendues comme telles au départ, et qui sous-tendent des changements de blocs harmoniques; elles sont signalées de façon très audible. Dans un premier développement, cette notion est préservée, mais j'introduis des séquences de rythmes avec des petites notes qui induisent des longueurs différentes : il n'y a plus, comme au début, une différenciation purement harmonique, mais une différenciation rythmique. Dans un autre développement, les petites notes interfèrent à l'intérieur de cette figure : on perçoit sa régularité par rapport à l'irrégularité des petites notes, et le tempo doit ralentir fortement. Ainsi, cette figure a pris différents sens par l'emploi technique qui en a été fait. C'est cela qui m'intéresse: que la thématique prenne un sens différent suivant son emploi, suivant le tempo par exemple. Une figure comme celle-là apparaît dans l'ensemble de l'œuvre: les éléments reviennent dans une forme en spirale, mais non à intervalles réguliers. On doit pouvoir les reconnaitre et, parfois, ne pas être sûr de les reconnaître.

22 - N'y a-t-il pas là, à travers l'élément thématique, une reconquête de la dimension du temps par rapport aux œuvres des années cinquante, qui étaient davantage basées sur des concepts de spatialisation que sur des concepts proprement temporels? 
Pierre Boulez : Oui, absolument ! J’ai appelé cela le temps indéterminé. Les dimensions des œuvres de l'époque étaient toutes variables, on avait l'impression de découper des tranches de temps arbitraires: on pouvait les faire plus ou moins courtes, plus ou moins longues... Ce qui, dans une telle situation, me paraît intéressant (je désire le réutiliser de façon intentionnelle), c'est qu'à un moment donné, les formes ne sont pas nécessaires; elles n'ont plus ni début ni fin. J'ai utilisé cela dans Répons à un moment où les solistes interviennent avec un accord de fond, qui subit des modifications de timbres pendant huit à dix minutes. Les machines déroulent cet accord selon un certain automatisme, mais on ne l'entend pas forcément : il apparaît quand les solistes jouent suffisamment fort, et disparaît à d'autres moments ; c'est comme si l'on ouvrait et l'on fermait alternativement une fenêtre sur cet accord. C'est une situation amorphe. J'aimerais, dans cette perspective, développer la conjonction du formel et de l'informel.

24 - Comment se fait-il qu'ayant reconquis cette dimension du temps, vous n'arriviez pas à trouver une conclusion aux œuvres que vous avez composées dans les dix ou quinze dernières années?

Pierre Boulez: A cause de la notion de spirale, d'un temps "infini"... Les formes que j'écris actuellement ont cette tendance : là où elles s'arrêtent, elles sont complètes, mais je peux ajouter un autre anneau à la spirale, et c'est encore complet. Dans Répons, par exemple, la fin restera identique, car il faut un signe final. Mais je la déplace au fur et à mesure, et je sais comment l'œuvre va se poursuivre.

26 - Pourquoi, dès lors, passer à une autre œuvre avant d'avoir mis le dernier anneau à celle que vous composez?

Pierre Boulez : Parce que je suis poussé par les nécessités. Eclat/Multiples, par exemple, a été abandonné au moment où j'avais beaucoup de travail comme chef. Mais cela reviendra : j'ai déjà deux fois plus de musique que ce qui est disponible! C'est écrit, à part la fin justement !

28 - Vous dites que "l'obsession du constant renouvellement de la chose proférée entraîne la perte du message"...

29 Pierre Boulez :... Cela se rattache à la question de la mémoire. On ne peut pas percevoir quelque chose qui est sans mémoire. S'il y a constamment des messages nouveaux, et qu'on ne puisse pas les référer à des antécédents, on perd tout intérêt. C'est très simple: ou bien vous avez des informations trop prévisibles, et vous vous ennuyez parce que vous savez comment les choses vont se passer, ou bien vous avez beaucoup trop d'informations imprévisibles, et vous vous ennuyez aussi parce qu'une information de plus ou de moins ne vous intéresse plus, vous ne pouvez pas être responsable vis-à-vis d'elle. Ce qui est important, c'est d'avoir une information mesurée permettant de s'intéresser à ce qui va suivre, de rapporter une information à quelque chose d'antérieur, ou de savoir qu'une information nouvelle prendra son sens plus tard. Il y a une sorte d'allée et venue de la perception et de la mémoire dans l'œuvre. Cette préoccupation, chez moi, existe déjà dans le Marteau sans maître. Dans la seconde pièce, commentaires de "Bourreaux de solitude", le début régulier est limité dans la dynamique; le milieu est très déséquilibré au point de vue des dynamiques (le xylophone joue dans les extrêmes avec des rythmes inégaux) ; la fin combine les deux écritures. Les deux seules œuvres qui sont vraiment volontairement indifférenciées sont Structures/ (et encore, la seconde pièce cherche à créer des différenciations) et Polyphonie X. Mais on a vu que la perception, dans de tels cas, ne marche pas! 
30 - Il me semble qu'à cette époque, il y avait chez vous une volonté d'objectivation du discours musical, un besoin, qui existe toujours, me semble-t-il, d'épurer l'œuvre de tout ce qui serait ambigu, hétérogène et non maîtrisé...

31 Pierre Boulez : Non, je crois que l'objectivité n'était pas notre principe d'existence. Elle a obsédé Stravinsky et, dans une certaine mesure, Ravel : mais ce n'était pas du tout notre position. Comme j'ai toujours détesté la musique néo-classique, cette espèce d'objectivation ne m'intéressait pas. J'ai toujours considéré, au contraire, que nous devions être subjectifs. Mais je voulais qu'il existe une pensée par rapport au langage : le langage ne s'invente pas au fur et à mesure, par des "coups de génie". Au contraire, c'est la mémoire qui fournit le coup de génie. Il faut aller radicalement dans la recherche d'un certain type de lois personnelles par rapport au langage, de sorte que ce que vous voulez dire soit dit d'une façon adéquate.

32 - Vous avez tout de même une démarche extrêmement rationalisante, qui évacue ce qui pourrait déranger, troubler l'ordre créé...

33 Pierre Boulez: Non, c'est une façon commode de résumer les choses! Ma pensée compositionnelle est beaucoup plus complexe. Les concepts aident à se diriger ; ils sont résumés d'une façon très rationnelle par rapport à l'emploi qui en est fait. C'est comme un guide : il ne vous fournit pas la saveur d'un pays que l'on veut visiter! Pour moi, les écrits théoriques sont comme une sorte de carte géographique par rapport à la réalité. C'est une réflexion en fonction de quelque chose d'autre; vous avez d'ailleurs pu observer que j'ai rarement fait des analyses moi-même!

34 - Pourquoi ne parvient-on pas à élever le discours verbal au niveau du sens musical, mais seulement au niveau d'un système qui peut produire aussi bien des chefs-d'œuvre que des œuvres mineures?

35 Pierre Boulez: Je crois que le jugement esthétique ne peut se formuler qu'en termes purement subjectifs, assez vagues d'ailleurs; il serait prétentieux de vouloir décrire une mélodie de Mozart pour ce qu'elle est : si on vous en fournissait les éléments, vous ne la trouveriez pas! C'est la même chose en littérature : ce n'est pas parce qu'on fera l'analyse grammaticale, sémantique ou rhétorique d'un poème, qu'on va en expliquer la beauté! Vous pouvez analyser une œuvre que vous ne connaissez pas, et devant laquelle vous êtes perdu, mais lorsque vous posez la question du "pourquoi", et plus seulement celle du "comment", vous êtes complètement dans le noir. Pourquoi le thème de la "Grande Fugue" est-il ce qu'il est ? Ce n'est pas le fait d'en dénombrer les intervalles qui vous le dira! Et le thème n'est qu'un élément: il faudrait parler de l'écriture, de l'architecture, et de toutes sortes de choses qui ne sont pas vraiment analysables! La seule analyse que j'aie publiée, celle du Sacre, était liée à mes préoccupations de l'époque: je crois que Stravinsky n'a jamais pensé, en écrivant l'œuvre, aux permutations de valeurs que j'y ai trouvées, et que je voulais y trouver. J'ai d'ailleurs dit, à l'époque, qu'une analyse ne pouvait servir que si elle était subjective !

36 - Que vous apprennent les analyses que l'on fait de vos œuvres?

37 Pierre Boulez: Je ne les lis pas: elles intéressent ceux qui les font, mais pas moi. Premièrement c'est du passé, et je ne tiens pas à me revisiter sans cesse; deuxièmement, cela ne me sert pas à grand-chose...

38 - Les termes "liberté de description" et "aura" qui sont apparus dans vos derniers textes, et qui consignent un espace de liberté d'invention par rapport à la rigueur du système, sont-ils précisément cette part de subjectivité qui échappe à toute analyse ? 
39 Pierre Boulez: La notion de "liberté de description", je l'ai trouvée avec le Marteau sans maître. Je n'ai plus conçu la série comme un ensemble de notes singulières, de points, mais comme des blocs d'accords. J'ai commencé par la troisième pièce, qui est monodique ; j'avais des blocs superposés qui étaient chaque fois différents et qui me permettaient de faire des mélismes à volonté, d'écrire les mélodies que je voulais, et de sortir du carcan de la suite de sons isolés. Ce qui m'a intéressé, dès lors, c'est le rapport entre des notes déterminées par un système, et celles qui ne lui appartiennent pas, que j'appelle des notes intersticielles. En écrivant à partir de systèmes très déterministes, où il n'y avait plus de règle verticale, nous avions perdu le sens de l'appoggiature, du retard, de l'anticipation, des notes étrangères à l'accord, etc. C'est-à-dire tout ce qui constitue un enrichissement du vocabulaire de base, et qui l'affecte, lui donne sens. Dans Répons, il y a, au contraire, énormément de ces éléments. Certains accords reviennent, mais avec des emprunts aux accords voisins; des figures s'inscrivent dans des registres parallèles mais avec des rythmes différents. Ce sont des structures qui sont déstructurées, qui sont travaillées de manière à acquérir une très grande souplesse d'emploi, et à accueillir toutes sortes de greffes. Par exemple, pour aller d'une note à une autre, j'invente des échelles qui acquièrent une fonction de liaison. C'est ce que j'appelle l'“aura" : la liaison d'une note à une autre, ou d'un accord à un autre, est faite par des moyens pensés sur le moment même, en fonction de leur insertion à l'intérieur d'un contexte donné. Dans Répons, toutes les cadences de solistes sont bâties sur de grandes notes pivots, et tout le reste est intersticiel, inventé à l'instant où j'en ai besoin : vous avez ainsi un tissu harmonique très cohérent sur lequel se développe ce tissu intersticiel.

40 - La structure harmonique renvoie-t-elle à un schéma préexistant ?

41 Pierre Boulez: C'est un schéma que j'invente au fur et à mesure, qui est pensé en fonction du développement. Cette architecture, qui est très forte, je la revêts de toutes sortes d'éléments qui la rendent plus complexe, plus intéressante.

42 - N’y a-t-il pas une part d'utopie dans le fait de devoir inventer à la fois "sa" langue et son propre style?

43 Pierre Boulez : On n'invente pas la langue à proprement parler : beaucoup d'éléments et de principes sont dans l'air du temps, ils sont non dits...

44 - Mais vous refusez catégoriquement ce qui est "hérité", vous avez toujours voulu couper de façon radicale avec le passé ?

45 Pierre Boulez : Les éléments hérités n'ont aucun intérêt, il faut les transformer ! Je suis très perméable à ce que les autres font ou ont fait, mais je l'incorpore. Je déteste le collage, les influences non digérées, etc. Ce qu'il faut, c'est absorber les influences, prendre leur substance, et s'en servir de façon personnelle.

46 - Comment se fait-il alors que vous dirigiez et enregistriez une œuvre comme Sinfonia de Berio qui est aux antipodes d'une telle position? Pierre Boulez : Je trouve cela très bien fait, très habile...

48 - C'est un point de vue artisanal...

49 Pierre Boulez: Oui, mais la tendance qui consiste à utiliser des citations ou des musiques populaires - par exemple, chez Berio, dans Coro, qui est déjà beaucoup plus complexe que Sinfonia - ne me touche pas beaucoup personnellement. J'ai besoin d'inventer les choses moi-même, ou alors il faut que ce soient des cultures très 
lointaines qui sont complètement coupées de moi: j'ai ainsi beaucoup pris aux musiques de Bali ou du Japon.

Ce qui m'a intéressé chez les Japonais, c'est leur conception du temps: je m'en suis approché, par exemple, dans la Troisième Improvisation sur Mallarmé, où il existe une influence très directe en ce sens, quoique très élaborée. Un passage de Répons a été influencé par des sonneurs de cloches en Angleterre qui font des permutations très intéressantes avec cinq ou six cloches - c'est une technique tout à fait passionnante! Mais je suis sûr que personne ne pense à cela! Moi seul le perçois.

51 - Pourquoi, en tant que chef, ne faites-vous plus beaucoup de créations?

52 Pierre Boulez : Faute de temps. Je crois que j'ai beaucoup donné, et je trouve que c'est maintenant aux autres d'agir!

53 - Mais vous "donnez" toujours beaucoup en vous occupant de différents organismes musicaux : qu'est-ce qui vous empêche de vous consacrer uniquement à l'écriture?

54 Pierre Boulez : Le fait que ces organismes existent, et il faut s'en occuper. Qui pourrait les assumer professionnellement? Si mon nom disparaissait, les subventions diminueraient. Il faut donc leur laisser le temps de s'affermir : on n'abandonne pas un jeune enfant aux intempéries! D'autre part, je n'aurais jamais pu écrire Répons si je n'avais pas créé ces organismes, car il n'en existe aucun équivalent ailleurs...

55 - Vous auriez écrit une autre œuvre...

56 Pierre Boulez : Oui, mais je voulais écrire Répons. Et cela a créé tout un mouvement de pensée qui m'oblige à faire un peu de "gymnastique".

57 - Quel est le programme de recherches que vous envisagez à l'IRCAM pour les années à venir?

58 Pierre Boulez: L'utilisation de plus en plus perfectionnée du temps réel, afin de parvenir, avec la technique, au niveau de la structure de la musique : qu'on ne soit plus obligé de simplifier la pensée. Faire aussi que la synthèse des sons soit plus perfectionnée et, enfin, que le langage symbolique qui sert au compositeur soit plus facilement compréhensible et manipulable.

59 - Le mélange des sources naturelles et artificielles vous semble toujours aussi inéluctable?

60 Pierre Boulez: Ah oui, parce qu'il y a beaucoup de choses que vous ne pourrez jamais faire avec les instruments! Si vous voulez utiliser des échelles changeantes de façon intentionnée, avec des intervalles inégaux ou plus restreints, vous êtes bloqué par toutes sortes de problèmes matériels, alors que votre oreille perçoit ces différences. Je ne considère pas du tout la technologie actuelle comme un fétiche, mais elle me donne la possibilité d'inventer des univers sonores qu'on ne peut obtenir avec des instruments traditionnels. Cette conjonction du nouveau matériau et de l'impulsion de l'instrumentiste - laquelle n'est pas chiffrable parce qu'elle comporte une grande part non mesurable - me paraît nécessaire. La nouvelle œuvre musicale, comme l'architecture nouvelle, proviendra d'un mélange de pensée et de matériau. A défaut d'une telle démarche, soit le matériau bloque la composition, soit la pensée est insuffisamment forte pour l'organiser. Et si j'ai une certitude, c'est bien celle-là !

61 - Comment appréciez-vous le fait que le monde intellectuel ne se soucie apparemment pas le moins du monde des problématiques musicales contemporaines? 
62 Pierre Boulez: Que voulez-vous que j'y fasse? Je ne peux pas me modeler sur les aspirations ou le manque d'aspirations des intellectuels ! Le dernier entretien que j'ai eu avec Foucault portait justement là-dessus, sur la paresse des intellectuels et sur le fait qu'ils s'accommodent fort bien d'une musique de fond au lieu de rechercher une musique qui corresponde au niveau de leur pensée. Voyez un journal représentatif comme Libération : quelle place offre-t-il à la culture, à la musique d'aujourd'hui, et plus encore à la poésie ? Car la situation de la poésie est peut-être encore plus frappante que celle de la musique : les deux derniers poètes qui sont lus ont pour noms Michaux et Char... Les autres ont un sort beaucoup plus triste!

63 - Vos propres références, sur ce plan, n'ont guère évolué : vous invoquez toujours les noms de Joyce, Char, Klee, etc.

64 Pierre Boulez: Dans le roman, par exemple, Butor a su apporter un renouvellement, mais cela ne s'est guère poursuivi. Les romans actuels sont très retardataires par rapport aux œuvres importantes de la première moitié du siècle ! Et un homme comme Claude Simon n'est plus très jeune! C'est pour cela que mes références ne changent pas beaucoup : si je voyais quelque chose de neuf, je sauterais dessus !

65 - Vous n'avez pas le même sentiment dans le domaine de la musique?

66 Pierre Boulez: Non. Je trouve que la musique est plus agitée. J'écarte évidemment le retour au romantisme qu'on trouve chez les Allemands ou dans l'école minimaliste, laquelle, selon Carter, correspond à une pensée très réactionnaire. Je place l'espèce de primitivisme des répétitifs au niveau de Carl Orff. C'est un peu comme la période de l'entre-deux-guerres : la musique y était d'une légèreté à ne pas exister ! Certes il y a cette tendance actuellement, mais en France moins qu'ailleurs. La musique y est encore une recherche, même si celle-ci est parfois simplificatrice. Le néo-romantisme n'est pas une recherche, il n'est rien du tout!

67 - Faut-il que la musique soit une recherche?

68 Pierre Boulez : Elle ne peut pas être réactionnaire. Il y a eu une période de recherche et de table rase après la guerre, qui était due en partie aux conditions sociales de l'époque. Mais, de toute façon, on ne peut pas rester en arrière. C'est toute la société qui veut cela, notamment à l'intérieur du domaine scientifique et technique, où il existe une évolution de la pensée extrêmement rapide, et où des changements radicaux ont lieu.

\section{NOTES}

1. Boulez Pierre, “Le Système et l'Idée”, in In Harmoniques n 1, Paris, décembre 1986, p. 62-p. 105. 


\title{
Entretien avec Iannis Xenakis
}

\author{
Philippe Albèra
}

1 Dans vos propres écrits, vous avez défendu l'idée que la composition devait s'appuyer sur des idées nouvelles plutôt que sur une quelconque tradition. Aujourd'hui, pensezvous toujours de la même manière?

2 Iannis Xenakis : oui, autant que possible. J'ai toujours cette même exigence. je dirais même que c'est une attitude nécessaire à un point de vue plus général.

3 - L'appliquez-vous à votre propre développement?

4 Iannis Xenakis : il le faut bien! Sinon, cela n'aurait pas de sens. Pour moi, faire de la musique veut dire inventer, créer totalement. Mais il existe toujours un acquis, et c'est là que la lutte commence : ou bien vous êtes laxiste, narcissique, et ce que vous avez fait vous plaît, ou bien vous désirez inventer quelque chose de neuf. C'est l'attitude que devrait avoir l'artiste, c'est une attitude naturelle de l'être vivant.

- Cela veut-il dire que vous remettez en question vos propres méthodes?

6 Iannis Xenakis : j'essaie mais c'est difficile. La trahison commence là : vous ne pouvez pas écrire une pièce, ne fût-ce que d'une minute, si vous devez tout inventer. On ne peut supprimer ce qui est acquis, même si vous le transformez ou si vous le transcendez. Voilà la tragédie !

7 - En écrivant une œuvre pour orchestre, tenez-vous compte des contraintes d'un tel organisme : sa hiérarchie, son fonctionnement, sa tradition?

8 Iannis Xenakis : la transmission de ce que je veux faire se réalise par l'intermédiaire de la notation, qui est un codage. Ce codage comporte une charge historique, stylistique et émotionnelle. Elle évolue, bien sûr. Or il est impossible d'utiliser l'orchestre sans ce codage. si je travaille avec des solistes, le problème est différent: les sonorités, les façons de jouer peuvent être modifiées parce qu'il existe une autre relation. La démarche la plus radicale est sans doute celle qui utilise les ordinateurs; mais cela reste théorique, car là aussi on rencontre des pièges à tout moment.

- Comment jugez-vous la recherche actuelle dans le domaine des ordinateurs?

10 Iannis Xenakis : A mon avis, elle piétine, et cela pour différentes raisons. L'une d'entre elles est que les compositeurs les plus originaux n'ont pas de formation scientifique 
adéquate. Mais le problème fondamental est dans l'absence de théorie: on travaille avec des conceptions anciennes, comme, par exemple, la synthèse additive, conceptions qui furent importantes mais dans un secteur déterminé. Il manque également des outils qui seraient à la mesure de théories fondamentales nouvelles. Pour parler concrètement: dans les années cinquante, j'avais proposé de travailler à partir des quantas sonores ; j'avais l'idée des nuages de sons, qui étaient une conséquence de mon travail sur la probabilité dans la composition musicale (musique stochastique). On m'a dit qu'il existait déjà une théorie mathématique due à Gabor (en fait, la théorie vient d'Einstein). Elle stipule que la transmission des vibrations qui produisent les sons, comme la transmission de la chaleur, ne se réalise pas de façon continue, mais par paliers; c'est la même loi que celle de la discontinuité de Planck. Mais je ne possédais pas les outils nécessaires, je devais tout faire à la main, et c'est très long! Il y a quelques années, de jeunes compositeurs, à Vancouver, à Marseille, et au MIT, se sont mis à faire de la musique avec des quantas sonores : je ne sais pas où ils arriveront, cela n'a pas vraiment d'importance, car c'est une exploration qu'il faut faire. Il s'agit donc là d'une théorie différente de celle de la synthèse additive qui provient de Fourier. Et il y aurait d'autres possibilités d'exploration à partir de théories situées en amont de l'utilisation des machines à calculer.

11 - Ce sont des théories sur le son, sur les outils, mais non, à proprement parler, des théories musicales...

12 Iannis Xenakis: Mais si, les choses sont liées. Pourquoi de telles idées sont-elles musicales, qu'on le veuille ou non? Parce qu'elles touchent aux questions de formes et qu'elles sont liées à des constructions d'objets sonores qui sont une sorte d'incarnation de ces théories. Si vous regardez un coucher de soleil, vous pouvez dire qu'il est beau, cela n'empêche pas qu'il s'agit d'un phénomène physique. C'est un peu comme les peintres qui utilisent des déchets : en les exposant, ils nous obligent à regarder des formes que l'on ne voit pas d'ordinaire d'un point de vue esthétique! Or tout est une question de formes ! Et les formes nous renvoient à la théorie.

- Le CeMaMu ne vous offre pas les moyens d'une telle recherche fondamentale?

14 Iannis Xenakis : Non, ses moyens restent limités et toujours précaires. Nous n'avons pas la certitude, d'une année sur l'autre, que les subventions seront reconduites, et à quel niveau. Le seul organisme qui puisse permettre cette recherche, c'est l'Etat, parce que, sans aucune utilité pratique, ni pour l'industrie, ni pour la guerre, cette recherche n'intéresse pas le privé... Il serait aussi nécessaire qu'il existe plusieurs centres de recherches : s'il n'y a qu'un seul laboratoire, avec un seul directeur, et que celui-ci se trompe, que se passe-t-il ? La science a progressé grâce à la multiplication des centres de recherches. Nous ne sommes plus au temps des conseillers du roi !

- Pensez-vous que des outils naissant d'une telle recherche remplaceront progressivement les instruments traditionnels?

Iannis Xenakis: Lorsqu'on fait de la recherche fondamentale, on ne se soucie pas de savoir si cela a des retombées commerciales ou grand public. C'est comme un artiste qui travaille sur un concept. C'est l'attitude qui m'intéresse. D'autre part, il ne peut y avoir de retombées commerciales ou pédagogiques que s'il existe une véritable recherche fondamentale. Les essais de composition musicale par le calcul, pratiqués au début des années cinquante, constituaient une objectivation, une recherche fondamentale; aujourd'hui, elle est détournée par des gadgets et par la facilité technologique. On en est encore à une ébauche de recherche en ce domaine. Lorsque j'effectuais mes calculs 
de probabilités, je me demandais : cela va-t-il marcher, cela a-t-il un sens esthétique ou non ? Il y aurait un article critique à écrire sur le travail que nous avons fait alors.

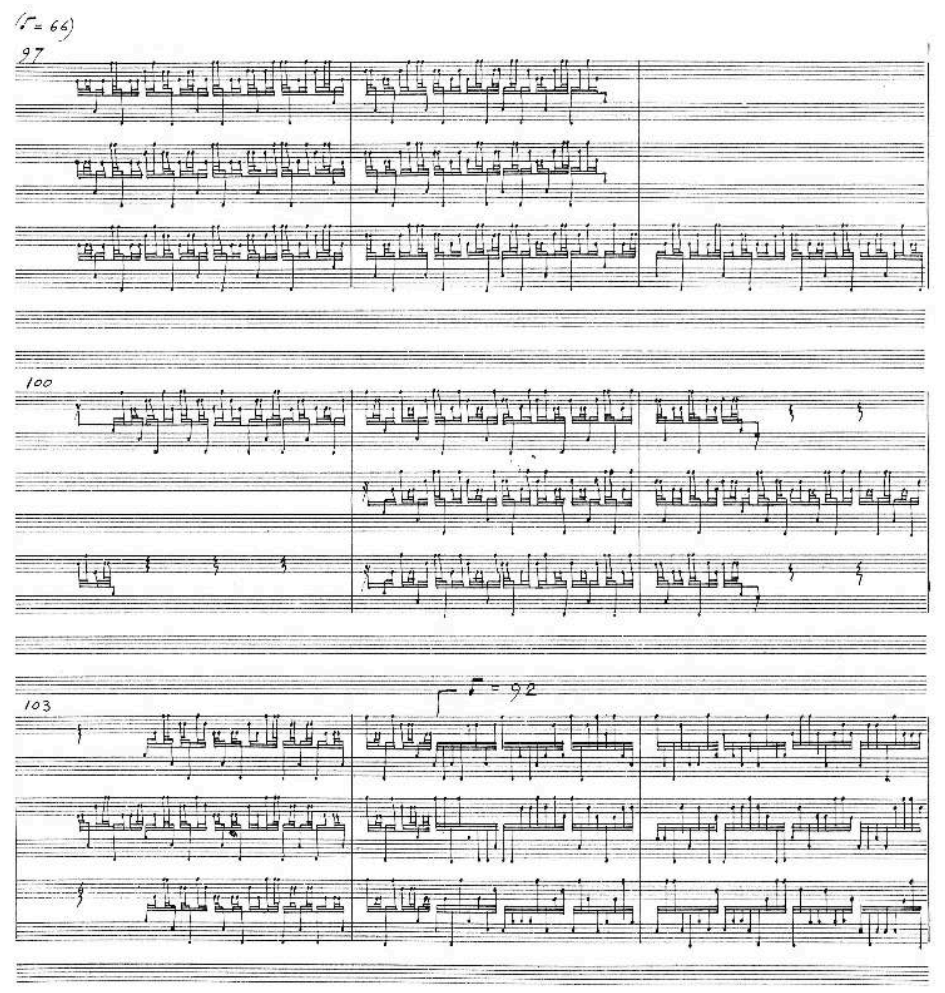

- Lorsque vous travailliez ainsi, aviez-vous une image sonore de ce que vous vouliez réaliser, ou, un peu à la manière de Cage, vous attendiez de voir ce que cela donnait ?

Iannis Xenakis : C'est trop compliqué pour voir simplement ce que cela donne! Il y a trop de travail! Composer une pièce de quelques minutes me demandait des mois de travail. Ce faisant, je formais mon goût, et c'est aussi l'intérêt de la démarche. Je travaillais à partir d'idées philosophiques, historiques, esthétiques. J'essayais d'imaginer autant que possible le résultat sonore. Lorsque j'ai écrit Metastasis, avec tous les glissandi de cordes, je suis allé voir Nadia Boulanger : elle a mis la partition sur le pupitre du piano, et m'a dit que c'était impossible à jouer, bien que cela semblât intéressant ! Il fallait que le résultat soit d'avance concluant à l'oreille, sinon cela aurait été avorté. C'est donc un risque que l'on prend plus ou moins consciemment : on essaie d'imaginer le résultat, mais avec un certain flou. Et même si vous prévoyez tout, vous prenez le risque d'avoir tort.

19 - Comment envisagez-vous, aujourd'hui, l'ensemble de ces idées, de ces utopies, aussi bien architecturales que musicales?

20 Iannis Xenakis : La plupart des choses se font parce qu'il y a une tendance. Vous pouvez proposer quelque chose qui reste dans un tiroir, parce que vous n'avez pas eu les moyens de l'exploiter. C'est un problème de mode et d'environnement. Ensuite se pose une question de moyens pour que l'on puisse aller de l'avant. Les idées demandent du temps! Il y a des rêves que l'on finit par réaliser, d'autres qui ne sont même pas envisageables...

21 - Pour vous, le compositeur doit-il se doubler d'un scientifique?

22 Iannis Xenakis: A mon avis, oui. La musique, dès l'Antiquité, était liée aux mathématiques et à la physique. La grande théorie de la synthèse additive, qui pénètre 
aujourd'hui dans tous les domaines de la science, provient de la musique. La musique a toujours été proche de phénomènes très complexes qui sont liés à la sensation: on perçoit par exemple les hauteurs non pas par un comptage, mais par les décharges des neurones électriques, qui sont de type statistique ; c'est ainsi que les choses se passent dans notre cerveau! Il est fantastique de penser que des notions scientifiques comme celles-ci existent depuis longtemps dans l'oreille, et que les musiciens l'ignoraient. Les musiciens ont fait des inventions qui étaient à l'avant-garde des mathématiques, mais sans le savoir! Et dans les Conservatoires, ils l'ignorent toujours! Par exemple : les quatre formes d'une mélodie forment un groupe de transformation isomorphe, c'est-àdire égal au groupe géométrique du rectangle. Il existe une proximité entre ce type de pensée géométrique en musique et la combinatoire des symétries qui se trouvent dans le monde qui nous entoure, et qui va jusqu'au domaine quantique (on ne décrit les particules qu'à l'aide de groupes). Un autre exemple : la notation. Vers le IX ${ }^{\mathrm{e}}$ siècle, elle est devenue neumatique (de "neume", le geste), après avoir été alphabétique, et cela aussi bien en Occident qu'en Orient : les premiers qui ont, d'une manière consciente, rattaché l'espace à la hauteur et au temps, deux dimensions qui n'ont rien à voir avec l'espace à trois dimensions, sont d'abord les grammairiens attiques qui ont inventé les accents pour conserver une langue qui se perdait. Au IX siècle, cette spatialisation à deux dimensions de la musique devient plus précise avec Guido d'Arezzo : c'est, en réalité, une prémonition de la géométrie analytique d'Oresme et de Descartes, quelques siècles avant. Une traduction du temps et de la hauteur en espace. Or on pourrait se poser la question: de quel droit fait-on ce transfert? En fait, il s'agit d'une structure mentale appelée structure d'ordre total: lorsque vous avez trois valeurs, vous êtes capable de dire que l'une des trois se trouve entre les deux autres.

- Vous voulez dire que les modes d'appréhension du réel saisis rationnellement par la science ont été découvert intuitivement par les musiciens?

24 Iannis Xenakis: Tout à fait. Pour des raisons pratiques et d'imagination. Mais aussi à cause de la paresse : les quatre formes de la mélodie en sont un exemple, car on ne peut pas tout inventer à chaque instant! Et c'est bien là le drame! Il faudrait poser d'une façon beaucoup plus générale le problème de la redondance, parce qu'il concerne la structure de notre univers, de nos relations sociales...

- On peut dire aussi qu'une invention perpétuelle s'assimile rapidement à un désordre... Iannis Xenakis : Et alors? Le désordre est-il mauvais en soi ? J'ai introduit le désordre statistique dans mes œuvres, mais par le calcul, pour organiser des masses. L'idée de masse est en principe désordonnée. Le désordre est quelque chose de très beau à voir : regardez le Cuirassé Potemkine de Eisenstein, qui a su tirer parti de cela!

- Mais, à partir du moment où l'on en a perçu les limites, ces masses ne constituentelles pas à leur tour un ordre?

Iannis Xenakis : Oui, c'est vrai. C'est comme la distinction entre bruit et son musical: dans le langage de Fourrier, le bruit blanc, ce sont toutes les fréquences avec n'importe quelle intensité changeant à chaque instant. La perception de ce bruit est globale. Tout cela fait partie du jeu à la fois conceptuel et esthétique. En musique, nous figeons dans notre mémoire les éléments perçus dynamiquement : on crée ainsi des contrastes entre ce qui est ordonné et ce qui est désordonné, entre complexité et simplicité, entre vitesse et quantité d'informations, etc. La musique a toujours expérimenté ces catégories. 
29 - Si la musique doit à nouveau être liée à la science, est-ce une rupture avec la manière dont nous la concevons, la pratiquons et la percevons depuis le XVIII ${ }^{\mathrm{e}}$ et le XIX ${ }^{\mathrm{e}}$ siècle ? Iannis Xenakis : Il serait intéressant de pouvoir réellement comprendre les musiques du $\mathrm{XVIII}^{\mathrm{e}}$ ou du XIX ${ }^{\mathrm{e}}$ siècle; on ne peut pas les comprendre, parce que les terminologies, les approches, viennent de l'intérieur. Dès lors, il s'agit d'une tautologie, vous ne dites rien du tout. Si c'est de l'extérieur, il s'agit de sentiments, etc. Il faut donc inventer des théories nouvelles: par exemple, des approches statistiques! On peut envisager certains objets musicaux non pas avec le langage des musiciens, mais avec des outils conceptuels qui viennent de la science.

- Il faut donc que la théorie soit extérieure à la chose étudiée ?

Iannis Xenakis: Oui, parce que, si l'on continue à faire de la musicologie comme aujourd'hui, cela ne conduit nulle part! Il faut l'étudier comme un objet. On sait bien depuis Aristote qu'il est impossible de démontrer la validité esthétique des œuvres! Il faut donc rénover les outils d'analyse. On pourrait, par exemple, utiliser la théorie des groupes pour étudier la musique fondée sur des intervalles et des durées définies. Moi, j'aime beaucoup la musique de Brahms : je peux très bien repérer telle cadence, tel renversement, telle construction polyphonique; mais je ne peux pas vraiment dire pourquoi j'aime cette musique ! Je n'ai pas les outils pour cela.

33 - Et que devient l'interprétation, au-delà de l'analyse "objective" ?

34 Iannis Xenakis: Ce que je veux dire, c'est qu'il faut forger des outils nouveaux pour comprendre d'une manière plus générale... Cela amènerait une élévation du niveau de l'art, de l'analyse et de la théorie. Je crois que la musique est plus universelle que ce qu'on veut croire aujourd'hui.

35 - Le rattachement de la musique aux sciences ne comporte-t-il pas une part mystique, le désir de retrouver, derrière les systèmes musicaux, une explication du monde? Iannis Xenakis: Je ne pense pas que ce soit une mystique. Regardez la musique de Bach : par-dessus ce que j'appellerais une rigueur froide et rationnelle, il inscrit des lignes vocales qui expriment la joie ou la tristesse et qui correspondent à quelque chose de tout à fait universel. C'est cet amalgame qui crée de la valeur. Lorsque le christianisme s'est imposé en Occident, la science, l'attitude de découverte et de discussion ont disparu, remplacées par le dogme, par la mystique, qui a donné très peu de choses, finalement. Il ne s'agissait pas de discuter, mais de croire. Le dogme a régné en Occident - comme en Orient - pendant des siècles, jusqu'à la Renaissance (appelée ainsi parce qu'on a renoué avec l'esprit antique). Ce qui fut alors intéressant, c'est cette fusion, ou cette dialectique entre les choses senties, irrationnelles, et ce qui est rationnel. Lisez ce que disent de grands mathématiciens : lorsqu'ils parlent de théories nouvelles, ils sont dans l'inquiétude parce qu'ils ne savent pas quelle en est la portée. Ils ont d'abord une intuition, une vision, et c'est ensuite qu'ils essaient de mettre cela dans un langage rationnel qui soit convaincant pour tout le monde.

37 - Cette idée que vous avez de la musique n'est-elle pas en contradiction avec le fait qu'on la perçoit à un tout autre niveau, y compris chez les intellectuels et les chercheurs?

Iannis Xenakis: C'est vrai, nous sommes infestés par la musique pop, la musique de variétés, et à un moindre degré, par la musique du passé. On peut faire la comparaison suivante : un chercheur de laboratoire découvre des substances chimiques. Il n'a pas à proprement parler une finalité. Mais il vit dans un environnement qui se saisit de sa 
découverte et qui la transforme en produit pharmaceutique, par exemple. En musique, les découvertes effectuées dans le domaine technologique se retrouvent, souvent très édulcorées, dans certaines musiques pop ou de variétés. De la même façon, ces musiques reprennent des éléments de langage venus de l'Inde ou de l'Indonésie, par le biais des guerres coloniales notamment.

- Quels sont vos contacts avec les philosophes, les scientifiques?

40 Iannis Xenakis : Lointains. Nous n'avons que des échanges ponctuels, rares. Je préfère communiquer par l'intermédiaire de leurs écrits! Il existe parfois des problèmes graves de compréhension mutuelle : je ne comprends pas les mathématiques de la même façon que les mathématiciens! Nous n'utilisons pas tout à fait le même langage. Ce qui explique, par exemple, que les scientifiques aient de la peine à entrer dans le monde de la musique contemporaine. Le scientifique qui, après une journée de travail, se délasse en écoutant de la musique de variétés, est tout de même loin de nos préoccupations. Je connaissais à Indiana un groupe de mathématiciens qui avait formé un quatuor à cordes: ils refusaient de jouer la musique de ce siècle. Ils n'interprétaient que des œuvres de Mozart ou de Beethoven, bien qu'ils fassent des mathématiques modernes !

41 - L'élément de la nature est chez vous un référent fondamental, y compris dans certains de vos concepts - nuages de sons, arborescences, etc.

42 Iannis Xenakis: Je connais mieux les choses de la nature que celles du corps par exemple. En tant que musicien, je ne pense pas que l'on puisse être indifférent à la nature qui nous entoure, même si nous vivons dans les villes, c'est-à-dire dans des grottes chauffées. La nature est un répertoire de formes, elle offre des prémisses de formations qui nous mettent sur telle ou telle voie. C'est un alphabet qui nous est assez clair.

43 - Comment s'élabore une composition pour vous : à partir d'une idée globale?

44 Iannis Xenakis : Aussi bien d'une idée globale que d'une idée élaborée par un travail perpétuel souvent obscur. Cela avec comme toile de fond l'idée qu'il faut se débarrasser des idées préconçues, et trouver des idées neuves.

45 - C'est-à-dire, concrètement ?

46 Iannis Xenakis: Je ne sais pas. C'est le mystère. Car souvent les idées neuves ou les réalisations neuves ne sont pas perçues comme telles. Il y a des idées qui viennent, et que l'on juge tantôt bonnes, tantôt non.

47 - Qu'est-ce qui vous détermine à apporter de telles réponses?

48 Iannis Xenakis : Dieu seul le sait! Ecrire une œuvre, c'est comme entrer dans un terrier. On peut avoir, comme porte d'entrée, une forme sonore, une idée abstraite, une couleur, ou bien rien du tout...

49 - Qu'est-ce qui vous intéresse dans la production actuelle?

50 Iannis Xenakis: Les compositeurs que l'on regroupe sous le terme de "musique spectrale", parce qu'ils essaient d'échapper aux timbres donnés par les instruments traditionnels, même si je trouve qu'il y a dans cette musique un défaut de structure : ils procèdent par taches sonores, et c'est insuffisamment dynamique. J'aime bien aussi les démarches de Dusapin ou François-Bernard Mâche, qui me paraissent originales.

51 - Et dans votre propre génération? 

vous ne leur apportez rien. Or, aujourd'hui, il existe une sorte de réserve, on ne siffle plus les œuvres nouvelles, et il est difficile de prendre pour critère la réaction du public. On ne sait pas dans quelle mesure il est préparé par les mass media, dans quelle mesure il est ou non spécialisé... J'ai monté, il y a deux ans en Sicile, l'Orestia devant près de douze mille personnes, et ce fut un grand succès. Mais il est difficile de dire pourquoi : était-ce à cause du lieu, du cérémonial, de l'histoire du village où avait lieu l'exécution, etc.? Au temps où je prenais des cours chez Messiaen, le scandale constituait presque un critère de vérité pour son propre travail. Aujourd'hui, nous en sommes loin.

Mais ce qui me paraît important pour un compositeur, c'est que sa musique soit jouée sans son propre effort, sans sa propre intervention, ou sans le jeu des échanges internationaux, etc. Lorsque vous écrivez une œuvre pour un interprète et qu'il continue à la jouer, c'est un critère objectif. Car l'interprète affronte le public, il sent l'œuvre de manière différente que le compositeur. Pour moi, c'est le critère le plus sérieux de la validité d'une œuvre, et il est finalement plus précieux que la réaction du public, qui peut toujours se tromper. Le fait que des interprètes, jeunes ou moins jeunes, s'intéressent réellement à ce que j'écris, est pour moi une sorte de récompense. 


\title{
Entretien avec György Ligeti
}

\author{
Philippe Albèra
}

1 Vous m'avez dit être gêné par le contexte dans lequel s'inscrit le questionnaire qui vous a été envoyé...

2 György Ligeti : Oui. Les questions que vous posez sont bonnes en soi, mais à l'intérieur d'un contexte culturel qui n'est pas le mien. Je pense qu'il existe des esthétiques différentes selon que l'on appartient aux cercles musicaux de Paris - qui sont aussi ceux de l'Allemagne, de la Suisse, et de l'Italie du Nord - qui partagent un certain idéal, une certaine idée de la modernité, ou à ceux des Etats-Unis, par exemple. Personnellement, je suis très attiré par certaines musiques ethniques, et j'ai développé un grand intérêt pour des structures rythmiques qui sont très différentes de celles de l'avant-garde européenne. Je me sens également éloigné d'une certaine conception "sociale" de la musique moderne, qui trace des limites infranchissables entre la musique que l'on dit sérieuse et les musiques pop, rock, de variétés, etc.

3 En vérité, je ne me sens plus appartenir - et peut-être n'ai-je jamais appartenu - à ce que l'on a appelé l'avant-garde, celle de l'époque de Darmstadt ; je ne me sens pas non plus lié au courant post-moderne où l'on a voulu m'enfermer depuis quelques années. Des œuvres récentes comme les Etudes ou le Concerto pour piano ne correspondent ni aux critères de l'avant-garde, ni aux critères des mouvements néos et ni à ceux de la musique minimale. Je partage cette situation avec de nombreux jeunes compositeurs je pense avant tout à mes élèves, bien que je ne me considère pas du tout comme un chef d'école.

4 Dans ma classe, nous avons beaucoup analysé et discuté les différentes cultures ethniques où existent des polyphonies très complexes : en Afrique, la musique subsaharienne comme celle des Banda Linda, la musique de cour de Buganda, celle des lamellophones du Cameroun, et puis la musique du Pacifique Nord, de la Mélanésie,... Je ne veux pas utiliser ces musiques comme un folklore, mais je suis très influencé par la pensée musicale qui les sous-tend. Je suis également très influencé par certaines recherches dans le domaine de l'art visuel.

5 - Comment s'effectue votre travail sur ces musiques : par l'écoute, par l'analyse, par un travail de transcription? 
6 György Ligeti : Je les écoute beaucoup, mais je ne fais pas de transcription, comme Bartok avec la musique populaire hongroise ou Messiaen avec les chants d'oiseaux. La première et la dernière Etudes pour piano sont fondées sur une polyphonie rythmique très complexe; elle n'a rien "d'africain", mais j'utilise une pensée/technique qui provient de la musique africaine. D'autre part, j'ai lu différents travaux ethnomusicologiques, tels que ceux de Simha Arom, de Hugo Zemp, ou de Gerhard Kubik.

7 J'aime aussi beaucoup le jazz et la musique légère latino-américaine : la musique des Caraïbes et celle du Brésil. Ma connaissance des musiques africaines est également récente : j'ai été très impressionné par les Chants à penser des Gbáyá enregistrés par Vincent Dehoux, qui sont très simples, mais qui sont pour moi de la très grande musique, à l'égal de celle de Mozart. Toutes ces musiques m'aident à trouver une orientation esthétique, mais je ne les utilise pas en tant qu'inspiration directe.

$8 \quad$ - Cela vous aide à "sortir" d'un certain concept de modernité?

9 György Ligeti : Oui, tout à fait. Une autre de mes préoccupations depuis quelques années, c'est la musique des XIVe et XV ${ }^{e}$ siècles français, depuis Machaut jusqu'à Dufay, et notamment les œuvres presque oubliées de la fin du XIV ${ }^{\text {e }}$, tel que le Codex de Chantilly... Je les connais depuis peu: lorsque j'étais étudiant, je ne savais rien de la musique de Machaut. Je connaissais celle de Dufay ou de Ockeghem, dont on retrouve un peu la trace dans la conception polyphonique de mon Requiem, mais la découverte des musiques du XIV ${ }^{e}$ siècle est récente pour moi.

10 Une autre polyphonie qui m'intéresse est celle de la Géorgie, notamment à cause de certaines possibilités harmoniques qui ne sont pas fondées sur le chromatisme ou le diatonisme, mais sur des rapports de quinte, de tierce majeure et de septième mineure naturelles. Je retrouve d'ailleurs des préoccupations parallèles dans la musique de jeunes compositeurs français comme Grisey ou Murail, ou chez Claude Vivier; ils m'intéressent beaucoup. D'autre part, les systèmes harmoniques non naturels, à peu près équidistants, comme dans la musique de l'Afrique et de l'Indonésie, m'ont aussi influencé.

11 Je suis également très intéressé par la géométrie fractale et par les mathématiques. Mais, si j'apprécie par exemple beaucoup Xenakis, qui travaille toujours sur la base d'algorithmes, je suis tout à fait opposé à sa méthode : je l'accepte comme compositeur, mais ma pensée est différente. Si je devais définir mon grand idéal artistique, je citerais Cézanne au-dessus de tous les autres : parvenir à une "géométrie" à travers l'utilisation des couleurs, écrire une musique qui ne soit pas calculée, mais qui s'apparente au monde de la géométrie.

12 - Les sciences exactes, ici, sont une source d'inspiration...

13 György Ligeti : Oui, mais c'est aussi une méthode de travail. En ce sens, je crois que ma manière de penser ne correspond pas à l'esthétique de l'IRCAM, par exemple, même si j'ai une immense admiration pour Boulez.

14 - On vous a beaucoup reproché l'esthétique néo-romantique du Trio avec cor, qui est apparue pour certains comme un reniement de vos œuvres passées...

15 György Ligeti : Je crois que l'étiquette "nouvelle simplicité" qu'on a voulu mettre sur le Trio n'est pas très satisfaisante: on l'applique aussi bien aux musiques néoexpressionnistes qu'aux musiques minimales. Or j'aime bien la musique de Steve Reich, mais je me place dans une autre perspective. Certes, on peut dire que le Trio possède des traits post-modernes, au sens où l'on parle de post-modernité en architecture par 
exemple (une architecture que je déteste de plus en plus!). Je suis aujourd'hui un peu critique avec cet aspect de l'œuvre, tout particulièrement en ce qui concerne certains éléments formels-les constructions ABA, par exemple. Mais cette œuvre était pour moi une sorte de protestation vis-à-vis de l'avant-garde, et les journalistes m'ont immédiatement catalogué...

16 Si l'on écoute ce que j'ai écrit depuis les années soixante jusqu'au Grand Macabre, on peut remarquer une grande continuité dans mes préoccupations. La principale est l'idée qu'une forme musicale donne l'impression d'exister dans un espace doté d'une profondeur. La musique nous donne l'illusion que le temps n'est pas le temps, mais un espace. Chez moi, la musique est toujours à la fois processus et objet. En ce sens, mon Concerto pour piano n'est pas en rupture avec mes œuvres précédentes.

17 - La couleur harmonique de l'œuvre n'est toutefois pas sans réminiscences tonales...

18 György Ligeti : C'est vrai, et cela provient sans doute du mépris que j'ai envers le chromatisme. C'est pour cette raison que je fais beaucoup de recherches harmoniques ; je les fais sans aucun dogmatisme.

19 - Le chromatisme vous apparaît-il comme une impasse?

20 György Ligeti : Vous pouvez dire qu'une théorie est vraie ou fausse dans le domaine scientifique ; mais, en art, il n'y a que les critères du style. J'ai l'impression de travailler de la même manière qu'autrefois un Brunelleschi qui utilisait des éléments anciens dans son architecture : je ne travaille pas avec la tonalité, mais avec une quasi-tonalité. Dans mes Nonsense Madrigals, il y a quelque chose de l'idée de tonalité, il y a des centres, et je ne veux pas éliminer cela. C'est une réaction contre le chromatisme que j'ai moimême pratiqué dans Atmosphère par exemple.

21 En réalité, je me sens très éloigné de Schoenberg et de Berg, et très critique à leur égard. Mais ce n'est pas pour retourner au XIX siècle. Je pense qu'il faut se libérer de tout conformisme, y compris celui de l'avant-garde.

22 - Comment se fait-il que tous les compositeurs de votre génération refusent aujourd'hui ce terme d'avant-garde?

György Ligeti : Je refuse les classifications en général. Il existe aujourd'hui d'autres points de départ. Ce que je n'accepte pas, c'est l'utilisation de type post-moderne des éléments anciens, comme on le voit actuellement dans l'architecture. Par exemple, j'aime beaucoup, à Paris, l'Institut du Monde Arabe de Jean Nouvel, parce qu'il n'est ni moderne au sens devenu académique du terme - le style post-Bauhaus, post-Le Corbusier par exemple - ni post-moderne. Voilà ce que je refuse: une modernité devenue académique. Cela ne veut pas dire que je n'accepte pas les différents styles. Avec mes élèves, je travaille aussi bien Répons de Boulez, qui est éloigné de mon esthétique, que la musique de Steve Reich, qui est tonale. Je défends l'idée d'une liberté totale. Il n'y a aucune raison de revenir à quelque chose qui a déjà été fait ; aussi suis-je opposé au néo-expressionnisme par exemple.

24 Il y a trois compositeurs qui restent importants pour moi aujourd'hui : Debussy, Stravinsky et Webern. Ce que j'aime toujours chez Webern, c'est la clarté de ses constructions; ce que je n'aime pas chez Schoenberg, c'est son pathos. C'est évidemment une question de goût - je n'aime pas le pathos. On dit parfois que tout a été fait dans la musique, et qu'il n'y a plus de possibilités nouvelles, mais je ne suis pas du tout d'accord: il y a toute une vie nouvelle dans la science et la technologie d'aujourd'hui, par exemple. 


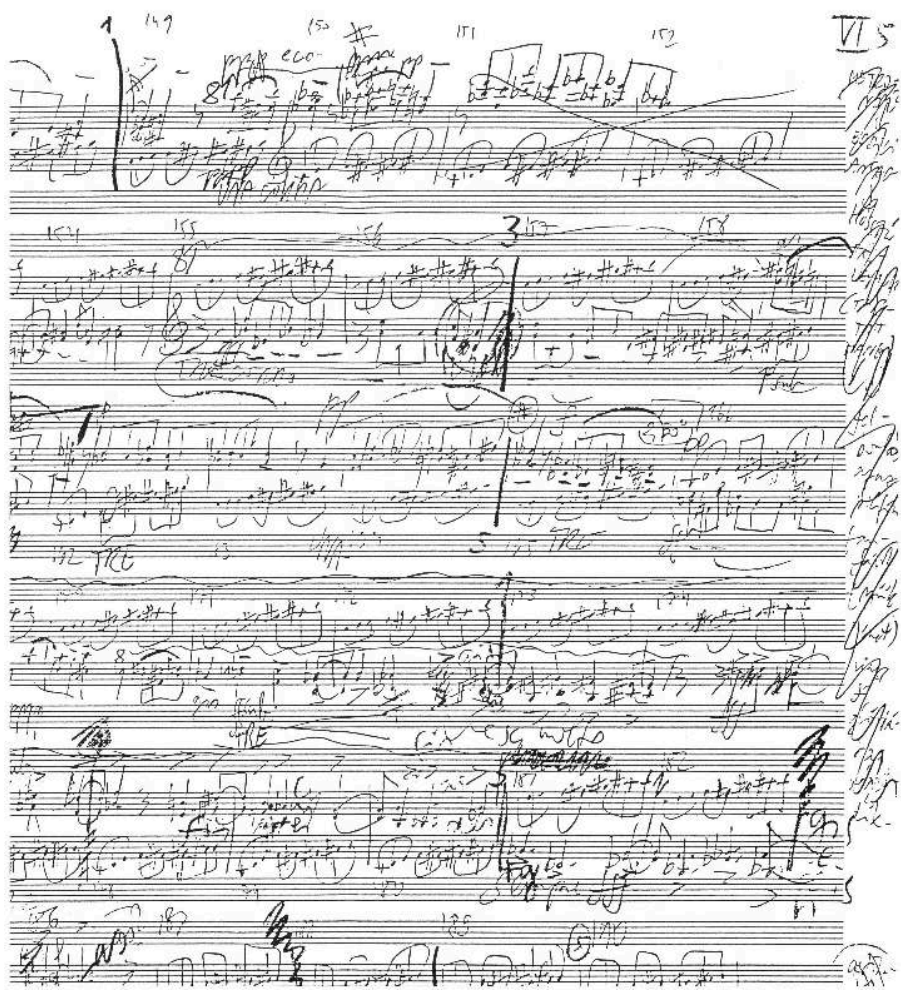

La géométrie fractale exige l'utilisation des ordinateurs. Dans le quatrième mouvement de mon Concerto pour piano, j'ai utilisé des formes qui présentent certaines analogies avec des structures fractales, mais je l'ai fait sans calculs. Je préfère travailler à la main : l'art ne doit pas être exact. Je m'inspire de données scientifiques, tirées de la géométrie ou des sciences naturelles, mais ce que je fais, c'est de l'art, ce n'est pas de la science.

- Ressentez-vous un conflit avec les instruments traditionnels ou avec les formes existantes?

György Ligeti : Il n'y a pas de conflit, car ce que je fais est fondé sur les instruments acoustiques. Je n'ai jamais voulu révolutionner les institutions ni les genres. Je cherche à faire une musique nouvelle dans les cadres existants. Mon intérêt se porte sur les contenus, non sur les formes.

28 - L'ironie qui existe dans certaines de vos œuvres, comme Aventures et Nouvelles Aventures, serait alors une manière de perturber l'ordre à l'intérieur des cadres traditionnels?

György Ligeti : Oui, si vous voulez. Je n'ai jamais pensé ainsi, je n'en suis pas conscient ! Dans Aventures et Nouvelles Aventures, je n'ai pas fait cela volontairement en tous les cas, contrairement à une œuvre comme Continuum pour clavecin, qui est une œuvre anticlavecin, puisque j'avais pris pour modèle une machine à coudre. Lorsque je compose, je pense en musique, mais je n'ai jamais d'intentions.

30 - Est-ce à dire que pour vous, une œuvre musicale n'a qu'une fonction esthétique ?

31 György Ligeti : Je ne me demande pas pourquoi je compose, ni dans quel but. J'en ai besoin. Ce n'est guère réfléchi! Je comparerais le travail du compositeur à l'activité d'un scientifique qui travaille dans la recherche fondamentale: il ne se pose pas la question des finalités, il suit un processus de recherche. 
32 Avec les médias, tout devient de plus en plus commercial; la vraie musique reste le privilège d'une élite intellectuelle. Elle l'a toujours été. Nous devons admettre que nous ne faisons pas de la culture de masse, même si Beethoven ou Dvorak en font partie dans une certaine mesure. Nous sommes à l'intérieur d'un tout petit cercle.

33 - Comment se fait-il, à votre avis, que la musique joue un rôle si faible dans l'ensemble du contexte culturel aujourd'hui ?

34 György Ligeti : Je ne suis évidemment pas qualifié pour répondre à une telle question : il faudrait pour cela s'adresser à des sociologues. Je ne peux qu'avancer des idées subjectives, sans prétention. Il existe à mon avis des conventions différentes selon les cultures et les pays : en France, c'est la littérature qui joue le premier rôle, c'est d'elle que l'on parle. En Allemagne, dans les derniers trois cents ans, c'est la philosophie qui a dominé; on le voit même par rapport à l'Autriche : le discours philosophique sur la musique y est presque plus important que la musique elle-même; il dicte certaines normes esthétiques.

Il y a toujours également une convention sur ce que l'on entend par le terme d'art: dans les cultures africaines, par exemple, la conception de la musique n'existe pas; on s'en réfère à la langue et à ses intonations, ou à la danse. Que, chez les Grecs et au Moyen Age, on ait associé la musique à une science, contrairement à la peinture, par exemple, n'était qu'une convention culturelle.

36 On pourrait se demander, par exemple, pourquoi les arts visuels s'imposent plus facilement que la musique aujourd'hui. Je crois que la raison est qu'il s'agit d'objets qui existent dans la réalité. Or, dans le capitalisme, cela représente une valeur. Ainsi, les arts visuels sont directement liés au commerce : même si l'on n'a aucune idée de l'art, on peut acquérir des objets d'art, et faire ainsi un bon investissement. La littérature et la musique ne peuvent pas entrer de la même façon dans ce grand système commercial. Je crois qu'au début du siècle, la musique a eu une grande importance à cause de l'institutionnalisation par la bourgeoisie d'organismes tels que les orchestres ou les quatuors. Cette institutionnalisation a changé la fonction de l'art. Le concert symphonique, par exemple, est devenu un musée. Alors qu'autrefois, les chefs étaient toujours présents à la tête de leur orchestre; aujourd'hui, grâce aux avions, ils se déplacent sans cesse d'un point à un autre de la planète. Cela a corrompu la vie musicale: on se contente de reprendre un répertoire qui n'exige pas trop de répétitions, que ce soit pour les concerts ou pour l'enregistrement des disques.

Il faudrait encore parler de la télévision : si elle laisse vivre la littérature et la peinture, en revanche elle tue la musique, qui y est utilisée comme bruit de fond. Le grand public, mais aussi les intellectuels, sont constamment entrainés à entendre la musique tonale ; au concert, ils acceptent la musique du XIX siècle, mais ils refusent celle de Schoenberg.

38 - Vos compositions s'élaborent-elles à partir d'une idée initiale globale ou se construisent-elles progressivement à travers le travail d'écriture ?

39 György Ligeti: J'ai toujours une imagination sensuelle, très directe, en musique. J'entends en général la globalité de la forme... Mais je peux aussi partir d'une idée abstraite: celle, par exemple, d'une grande complexité rythmique. C'est toujours quelque chose de global. Il y a une interaction de différents concepts : ce que je veux faire, ce que je veux éviter... J'ai écrit les Etudes pour piano, parce que j'aime beaucoup cet instrument et sa littérature; mais cela signifiait, pour moi, d'écrire une musique 
venant de la position des doigts sur les touches. L'idée première a été la joie tout à fait sensuelle de jouer, même si l'œuvre n'a rien d'une improvisation. lorsque je suis venu en Europe de l'Ouest comme réfugié, je n'ai pas eu de piano: je composais beaucoup dans les cafés ! J'ai disposé d'un piano à la fin des années soixante. Evidemment, si j'écris une pièce micro-tonale, comme le Concerto pour flutte et hautbois, qui était influencé d'ailleurs par Harry Partch, le piano m'est totalement inutile ; mais il m'arrive aussi de faire des recherches harmoniques au clavier.

- Vous composez au piano?

- Aujourd'hui qu'il n'existe plus de code harmonique, quel est le critère d'unité ou de cohérence en ce domaine?

György Ligeti : Dans certaines pièces, j'utilise le tempérament habituel, mais je travaille contre ses données, en évitant, par exemple, les combinaisons harmoniques chromatiques. Dans la première Etude, la main gauche joue sur les touches noires, et la droite sur les touches blanches, sans que cela ne produise jamais un total chromatique. L'effet est plutôt une espèce d'irisation entre la diatonie et la pentatonie. Toutefois, ne cherchez pas dans ma musique un concept théorique, ou un système harmonique. Mes idées ne sont pas fondées sur des systèmes, comme la dodécaphonie par exemple, mais elles s'inscrivent dans un espace toujours ouvert.

- Qu'est-ce qui vous détermine alors à éliminer certaines solutions?

György Ligeti: Les pensées musicales et esthétiques subjectives. Je ressens, par exemple, le besoin d'harmonies d'une grande luminosité dans le Concerto pour violon que je prépare actuellement : j'y travaille avec des intervalles tempérés ou non tempérés, avec les possibilités qu'offent les harmoniques, etc. J'ai toujours travaillé ainsi : à partir des données du timbre et de l'harmonie. Comme Cézanne, j'aime travailler les formes en partant de la couleur. Dans le troisième mouvement du Concerto pour piano, j'utilise essentiellement des combinaisons de quinte qui caractérisent toutes les sonorités. De tels modèles correspondent à une imagination personnelle.

46 Il y a des compositeurs pour lesquels le système est important, comme Stockhausen avec la forme-processus, ou Boulez avec un certain constructivisme, ou encore Cage avec l'utilisation du hasard. Dans ma musique l'unité et la cohérence demeurent cachées. J'ai une conception esthétique très claire, mais je me méfie des théories ; je ne recherche pas la sécurité.

47 Je ne travaille pas avec les catégories rythmiques de l'avant-garde, ou avec celles de Cage et de Feldman, qui sont toujours apériodiques. J'utilise plutôt des rythmes périodiques sur des pulsations très rapides et asymétriques. De ce point de vue, j'ai changé stylistiquement, même si la forme globale n'a pas changé.

- Faut-il voir dans ces préoccupations rythmiques l'influence de vos racines hongroises?

György Ligeti : Non, parce qu'on trouve cela plutôt dans la musique balkanique, la musique hongroise étant très simple de ce point de vue. Je crois qu'il s'agit plutôt d'une attitude: je n'accepte pas les normes restrictives, qu'elles soient politiques, philosophiques, musicales, mais je veux toujours tout remettre en question. Dit négativement: je suis le traître de la musique moderne! Je ne veux pas accepter les choses telles qu'elles sont, y compris dans ma propre musique. Je suis à l'opposé d'un artiste comme Yves Klein, qui après avoir trouvé le bleu l'utilisait systématiquement, 
ou de quelqu'un comme Cage, qui travaille avec une méthode de base. Je mets toujours en question mon propre travail, mon propre style.

- Avez-vous encore des discussions sur des questions techniques ou esthétiques avec les compositeurs de votre génération?

György Ligeti : Je vois parfois Boulez, Kagel, Cerha, Szöllösy ou Kurtág, et je les admire beaucoup. Nous déjeunons ensemble, mais il est rare que nous discutions de questions musicales.

- Ce débat ne vous manque-t-il pas par rapport à il y a quinze ans?

György Ligeti : Le contexte social des artistes a changé. Etait-ce vraiment différent auparavant? On parle du mouvement de Darmstadt, mais, pour une grande part, il s'agit d'un mythe. Il existait des positions musicales antagonistes, et certains indépendants, comme Nancarrow ou Scelsi par exemple, n'étaient pas du tout connus. On méprisait beaucoup de compositeurs parce qu'ils ne suivaient pas la voie principale. Cela crée aujourd'hui une perspective trompeuse. Ce qui a disparu, par contre, c'est le grand mouvement qui existait autour des radios: après la guerre, en Allemagne, les radios ont voulu promouvoir un art nouveau; elles diffusaient de la musique contemporaine et des conférences. Aujourd'hui, la musique légère a submergé tout cela.

54 - Comment enseigner en l'absence d'une "langue" objective? N'enseigne-t-on pas avant tout son propre style?

55 György Ligeti : Dans mes cours, nous discutons tous les styles! Il y a parfois un effet de feed-back amusant: ainsi, j'ai composé les pièces pour clavecin, qui sont très rétro, comme une réponse à certains de mes élèves qui avaient écrit une musique tonale à laquelle j'étais opposé : je ne voulais pas discuter avec les mots, et c'est pourquoi j'ai écrit ces pièces.

56 - A votre avis, la tradition, pour un compositeur occidental d'aujourd'hui, comprendelle les musiques extra-européennes?

57 György Ligeti : Pour certains compositeurs, je réponds oui. Lorsque Stockhausen veut faire la "musique du monde", c'est important, même si son approche est complètement différente de la mienne. Mon intérêt pour les musiques extra-européennes, comme celui que j'ai pour les mathématiques, ne veut pas dire que je suis ethno-musicologue ou mathématicien: il est en rapport avec mon désir de réaliser une polyphonie complexe et des formes musicales fondées sur des structures d'une grande complexité. Pendant quinze ans, entre les années cinquante et la fin des années soixante, je me suis concentré sur l'élimination du rythme, j'ai travaillé sur le timbre et la forme. Aujourd'hui, même si j'ai gardé le goût d'une musique "statique", je me concentre sur les concepts de processus et d'objets, ainsi que sur l'espace illusoire, qui est chez moi une idée fixe. Si je m'intéresse à certaines musiques extra-européennes et pas à d'autres, c'est en raison de mon obsession d'une complexité polyphonique à partir d'éléments harmoniques et rythmiques.

58 - Pourquoi la complexité ?

59 György Ligeti: En principe, la complexité n'est pas une valeur en soi : il y a des musiques très simples, comme celles de Schubert et de Moussorgsky, qui sont de très haute valeur. Mais j'ai une prédilection pour les formes de grande complexité. J'ai un amour pour les ornements labyrinthiques du Book of Kells et L'Evangéliaire de Lindisfarne, pour cet art irlandais du IX ${ }^{e}$ siècle, pour les ornements des Vikings et des Maori, pour 
l'art arabe comme celui de Grenade, et surtout pour les étonnantes représentations des ensembles Julia et Mandelbrot, réalisées à l'aide d'un ordinateur par Mandelbrot, Voss, Peitgen et Richter. Je suis en train de trouver une nouvelle musique orientée vers les objets fractals, vers les idées de la nouvelle science du chaos déterministe. 


\title{
Entretien avec Georges Aperghis
}

\author{
Philippe Albèra
}

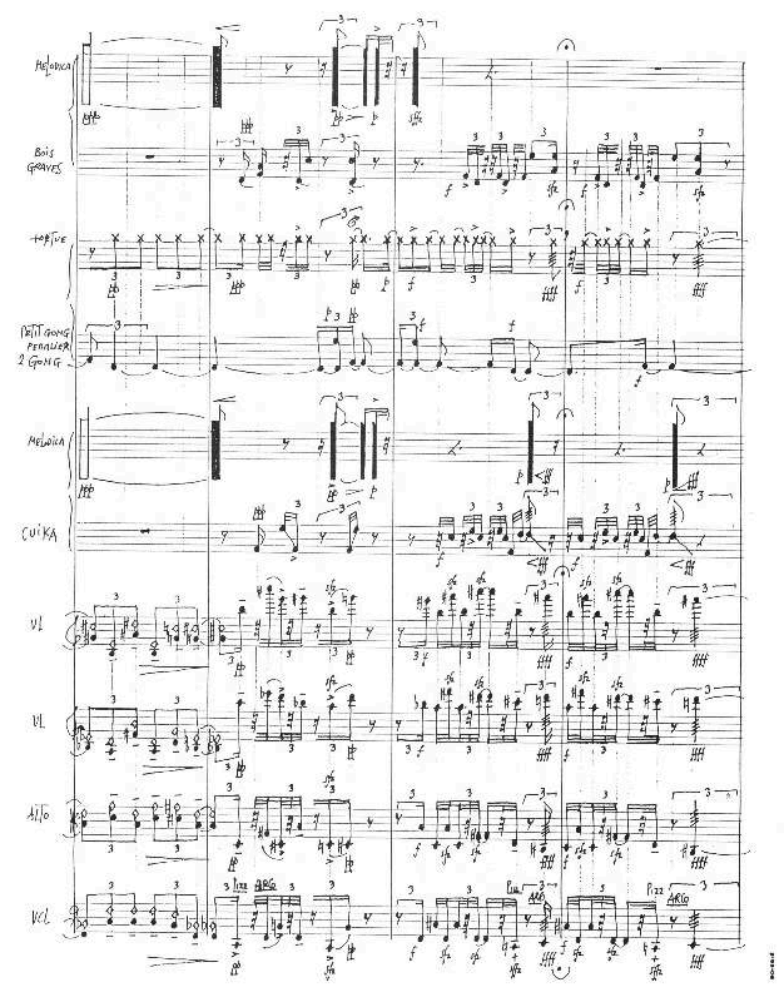

1 Votre musique semble n'avoir jamais été marquée par le mouvement sériel : quelle est votre position à ce sujet?

2 Georges Aperghis : Je me suis essayé au sérialisme pour voir comment cela fonctionnait. Ce qui m'intéressait le plus, c'était la manière dont le système produisait des aléas, qui ne permettent pas un contrôle immédiat sur la totalité de l'œuvre. Mais j'ai assez vite pensé que je pouvais arriver au même résultat avec des moyens qui m'étaient propres. En réalité, il y a peu de choses qui ne soient pas régies par la loi des séries. A partir du moment où vous créez un lien entre des éléments, une série se constitue, qu'il s'agisse d'un texte, d'une peinture, ou d'une œuvre de musique! Ce n'est donc pas la série en 
tant que telle qui ne m'intéressait pas, mais une manière de la traiter qui produisait une musique trop prévisible. Je ne l'ai pas utilisée à cause de cela. Ce qui me paraît plus intéressant, c'est de fabriquer mes propres systèmes, avec la musique qui en découle. C'est aussi plus gratifiant.

3 - Lorsque vous parlez de "fabriquer vos propres systèmes", est-ce en fonction d'une image musicale précise, ou afin de découvrir une musique inouïe?

4 Georges Aperghis : C'est très compliqué. J'aime avant tout être surpris. Donc, pour moi, fabriquer un système, c'est créer, à partir d'éléments connus, une combinatoire produisant des résultats inattendus. Ils sont inattendus par rapport à ce que l'on a dans la tête, bien entendu. Il ne s'agit pas d'un système général, ce que je n'aime pas beaucoup, mais d'un système particulier à chaque pièce, et qui demeure caché.

5 - Ce matériau déjà existant, ce sont des éléments d'écriture, des gestes, des caractéristiques de style...?

6 Georges Aperghis : J'essaie de retrouver la même immédiateté physique qui existe dans la plupart des musiques, et qui a été quelque peu perdue à cause de l'éclatement du début de ce siècle. Dans la musique "éclatée", ou "abstraite", comme je l'appelle, les événements qui surviennent dans le temps et dans l'espace sont comme distribués par une autorité supérieure - qui est souvent, d'ailleurs, une sorte de hasard contrôlé. Nous sommes face à un écran envahi par des sortes d'“accidents". Or la musique que j'essaie d'inventer ne vient pas d'en-haut, elle ne nous tombe pas dessus, mais elle est créée par l'instrumentiste. Ce que je cherche, c'est une musique qui sorte du corps, où l'on retrouve cet état physique entre le corps de l'instrumentiste et le corps musical. Cela peut devenir théâtral ou non.

7 - Précisément, est-ce cette conception qui vous a amené à l'idée du théâtre musical, ou est-ce le contraire?

8 Georges Aperghis: Je dirai que pour effectuer cette rupture par rapport à ce que j'appelle la musique "abstraite", le théâtre m'a beaucoup aidé, notamment parce qu'il met en valeur le corps humain.

9 - Ce qui voudrait dire aussi que vos références étaient très différentes de celles des compositeurs sériels par exemple?

10 Georges Aperghis : Non, c'est la même tradition, mais avec un point de vue différent. Je dois tout de même préciser que j'ai une grande admiration pour la musique que j'appelle "abstraite", même si je suis incapable d'en faire moi-même. Il fallait passer par là. Personnellement, je veux revenir à l'homme. Ce n'est pas la résurgence de la vieille opposition entre le corps et l'intellect: l'intellectualisme peut exister dans une pièce complètement physique, comme, par exemple, la Hammerklavier de Beethoven. Pour moi, Webern représente l'extrême limite d'une musique dont on peut entendre qu'elle est faite par des hommes.

11 - Est-ce qu'il n'y a pas, dans cette idée de système général, ou, comme vous dites, de musique "abstraite", un aspect mystique et spiritualiste auquel vous opposeriez une musique plus charnelle?

12 Georges Aperghis: Au contraire, les pièces qui m'intéressent le plus sont les pièces mystiques. C'est pour cela que j'aime beaucoup Webern! En réalité, je me sens très proche du mysticisme. Ce dont je me sens éloigné, ce sont des œuvres où n'existent plus que des structures dans l'espace et dans le temps, et pour lesquelles je ne parviens pas à imaginer un corps humain. Lorsque j'écoute les Klavierstücke de Stockhausen ou les 
Structures de Boulez, j'en comprends le projet, mais cela ne correspond pas à ma façon de sentir. En revanche, je suis comblé devant des peintures abstraites de Rothko par exemple. d'orchestre de nulle part, et cela m'intéressait de voir quelles possibilités d'union ou de conflits il en naîtrait. Je ne sais pas si la pièce sera bonne, mais je pense que c'est une bonne idée: les différences de comportement entre chaque groupe sont très intéressantes!

21 - Dans une pièce comme celle-là, vous commencez par répertorier l'ensemble des possibilités sonores des instruments, de leurs modes de jeu?

22 Georges Aperghis: Plus maintenant; je faisais cela auparavant. Je demande aux interprètes, selon mes besoins, ce qui est possible ou non. Il y a des problèmes que l'on ne rencontre pas lorsqu'on travaille avec des données abstraites, mais qui apparaissent avec des données physiques : par exemple, le fait que les sons soient forte ou piano, ou qu'ils tombent tous ensemble d'une certaine manière, exige qu'ils apparaissent dans un processus de dramatisation. Lorsque les événements ne sont plus régis par un cadre abstrait, ou par le hasard, mais lorsqu'on sent un corps qui respire et qui vit, il y a une dramatisation. Mon souci est que ce ne soit pas celle du dix-neuvième siècle ! C'est la même situation que dans le théâtre : la dramaturgie d'aujourd'hui n'a rien à voir avec celle de l'époque romantique...

23 - Votre travail dans le domaine du théatre est-il sur ce point une source d'inspiration, de stimulation? 
Georges Aperghis : Ce qui m'intéresse, c'est de voir dans les pièces du répertoire, ou les pièces nouvelles, les archétypes qui font le drame. Il existe des archétypes qui permettent de créer des conflits à l'infini - et je suis très intéressé de voir comment cela fonctionne. Par exemple, l'archétype de l'Orestie se retrouve plus tard dans Hamlet, puis dans La Mouette... Pourquoi dramatiser? Faut-il exagérer, jouer avec le texte ou contre lui? Je vois toujours ma musique comme quelqu'un qui vit et qui respire ; je me dis : il pourrait aller par là, ou par ici. C'est un parcours, c'est un voyage, qui joue beaucoup sur la mémoire: on reconnaît des choses que l'on a entendues, et qui ensuite se transforment...

- Quelle est la relation entre vos musiques de scène et vos œuvres de "musique pure" ?

Georges Aperghis : Les musiques de scène constituent des petits travaux qui permettent de souligner certains éléments dramaturgiques dans une pièce.

27 - Est-ce qu'ils forment des études pour ce qui pourrait devenir un nouvel opéra?

28 Georges Aperghis : L'opéra pose des problèmes pratiques et techniques énormes, liés notamment aux types de voix exigées, des voix capables de remplir un espace de 2000 places. Toutes les voix m'intéresssent, mais justement, pas uniquement celles-la. Ensuite, l'orchestre et les salles elles-mêmes posent problème : cela ne nous appartient pas, puisque c'est lié au répertoire, au passé. Il faudrait imaginer des salles moins colossales. Ce sont ces éléments-là qui sont problématiques, et non pas l'écriture proprement dite, qui demeure très intéressante. Je pense que l'on pourrait les résoudre par le film: le micro donne en effet des chances égales à des voix travaillées ou non travaillées, parlées ou chantées, utilisant des types d'émissions diverses, ainsi qu'à de nouvelles combinatoires instrumentales... Nous avons fait un film avec Enumérations qui me semble une voie intéressante pour ce que j'ai envie de réaliser maintenant. Je vois beaucoup de possibilités dans le fait d'écrire directement pour le film, avec les protagonistes dont j'ai besoin, sans structure préalable.

29 - Le théâtre musical n'est-il plus, à vos yeux, une alternative valable à l'opéra ?

30 Georges Aperghis: Tant que le théâtre musical se présente comme une alternative à l'opéra, cela n'a pas beaucoup de sens, parce que c'est un opéra avec moins de moyens. Ce qui est intéressant, c'est le théâtre musical en tant que genre nouveau. Si l'on utilise une seule personne, cela ne doit pas provenir d'un manque d'argent, mais de la nécessité du spectacle. Le théâtre musical ne doit pas être un opéra de poche : il doit être basé sur un système autre.

31 - Cette forme n'est-elle pas aujourd'hui dans une impasse?

32 Georges Aperghis: Non, je ne crois pas. Personnellement, j'ai très envie d'y travailler encore.

33 - Quelles sont les œuvres, ou les démarches, qui vous paraissent intéressantes en ce sens?

34 Georges Aperghis: Je ne sais pas, car je ne vois rien, je vais nulle part, ni au concert, ni au cinéma, ni au théâtre. Je suis très égocentrique! Lorsque je travaille, le fait de voir des spectacles me perturbe : cela me prend du temps pour y penser. L'important, pour moi, est que la dramaturgie soit musicale, qu'elle provienne de la musique et non du texte. C'est la partition musicale qui doit faire naître les images, les situations, le jeu des acteurs. En ce sens, cela n'a rien à voir avec l'opéra, qui est basé sur un texte 
préalable, des situations dramatiques, etc. Ce qui m'intéresse, c'est de voir jusqu'où la musique peut organiser un ensemble d'éléments divers.

- Si vous jetez un regard rétrospectif sur votre évolution, avez-vous l'impression d'une ligne continue, ou de mutations, de changements de direction?

Georges Aperghis : Je trouve surtout que mon évolution est d'une très grande lenteur. J'ai mis beaucoup de temps à comprendre certaines choses, car je suis très empirique : je ne crois pas à ce que l'on me dit. Lorsqu'on me démontre quelque chose d'un point de vue théorique, je n'entends rien. Et dix ans plus tard, je fais le même constat, mais par mon propre travail. Par exemple, en ce qui concerne le théâtre musical, je n'ai pas encore suffisamment de connaissances pour pouvoir écrire chez moi, et donner les partitions aux musiciens. Je dois travailler d'une façon empirique, sur place. En plus, je ne suis jamais sûr de rien. J'ai toujours peur de m'être trompé sur toute la ligne, que toutes mes idées, mes réalisations, soient basées sur une équation, et que cette équation soit fausse. Mon cauchemar, c'est de construire une fusée Ariane, avec toute la technologie nécessaire, mais sur une donnée de base qui est fausse.

- Quelle est cette donnée de base dans la composition?

Georges Aperghis : C'est difficile à dire : un amas d'idées, de savoirs, de désirs, que je ne pourrais pas définir. C'est d'ailleurs pour cela que j'écris de la musique! Je les vis en écrivant. Et je suis très heureux lorsque j'écris - ce qui est préférable, car j'écris toute la journée!

- Comment se déroule justement ce travail d'écriture?

Georges Aperghis : Je fais des plans, qui s'avèrent tout de suite caducs; donc j'en fais d'autres, j'aligne des idées, je reviens en arrière... Très souvent, je recommence tout deux ou trois fois pour arriver vraiment où je veux. C'est difficile à exprimer, car ce sont des choses indiscernables, c'est le contraire d'une affirmation : il s'agit de pousser les marges de deux ou trois idées antagonistes pour voir jusqu'à quel point leur friction est intéressante. C'est pour cela qu'il est difficile de définir le travail de composition. Par exemple, le mélange d'un quatuor à cordes avec un trio de percussions plus ou moins folklorisant, jouant des instruments venant du monde entier, chantant, hurlant, doit créer une friction : sinon, j'ai perdu mon temps. Je veux entendre cette friction, et non pas une jolie pièce de musique de chambre.

41 - Pourquoi cette pensée très empirique ne vous a-t-elle jamais poussé vers le domaine électro-acoustique?

Georges Aperghis: Je ne peux pas supporter les sons électroniques ou les sons synthétiques : c'est une réaction physique. Je ne leur accorde aucun crédit, comme s'ils n'existaient pas. En réalité, je pense que l'on doit être capable de tout faire avec une boîte d'allumettes. Si l'on me dit : voilà une machine avec laquelle tout est possible, je préfère aller planter des choux. Ce que j'aime se situe entre la tromperie et la vérité, ce sont des choses de la vie, faites avec rien. Lorsque j'écoute un son électronique, je me dis : bon, pourquoi pas un autre? Je n'arrive pas à prendre ces sons-là en compte. Et comme je déteste la science-fiction, je ne peux guère les relier à cet imaginaire-là. D'autre part, il faudrait que l'appareillage soit aussi simple à manipuler qu'une flûte de pan, ou que je puisse travailler sur des matériaux de récupération, sur des matériaux qui ont servi à autre chose. Il faut être capable d'étonner les gens avec une boîte d'allumettes. Si l'on a quarante consoles et que l'on ne peut pas créer une seconde d'émotion, c'est que l'on n'est pas un artiste. C'est la loi du théâtre ambulant, de 
l'accordéoniste du coin. Après tout, nous sommes là pour émouvoir un peu les gens, pour les faire penser. Pourquoi le son d'une flûte au milieu du désert nous émeut-il ? Le seul qui arrive à nous faire croire qu'il avait besoin d'une grande quantité d'instruments, c'est Mahler. Sinon, je ne marche pas... Si une œuvre comme la Missa Solemnis fonctionne, c'est que la pensée est tellement forte que, proportionnellement au gigantisme, elle gagne. Mais quand le gigantisme n'est pas à l'échelle de la pensée, cela donne un résultat totalement dépourvu d'émotion.

- Notre époque n'est plus capable d'une pensée suffisamment forte, à votre avis ?

Georges Aperghis: Nous n'avons pas de pensée globale, ou globalisante. Les grands monuments anciens, comme les messes, étaient liés à une pensée globalisante. Aujourd'hui, nous avons beaucoup d'informations partielles, nous voyons s'effondrer certains mythes, nous savons qu'il y a un peu partout des sens interdits, nous avons des morceaux de pensée... C'est pour cela que je travaille de cette façon: je ne vois pas comment je pourrais m'attaquer à quelque chose de monumental aujourd'hui ; ce serait une faute. Le morceau de flûte joué au milieu du désert a une cohérence absolue ; nous, nous avons cassé cette cohérence. C'est ainsi que j'en suis arrivé à l'idée d'Enumérations. Je me suis dit: de quoi sommes-nous encore sûrs, encore capables? D'énumérer des choses! Autrefois, les gens avaient quelques certitudes : par exemple, qu'il existait un paradis et un enfer, que le Parti Communiste était formidable, que la révolution était nécessaire au Vietnam, etc. Cela nous facilitait la vie.

- Vous vivez cette situation sur un mode tragique, ou au contraire vous êtes content que les masques soient tombés?

Georges Aperghis : Je suis très content. J'ai l'impression d'assister à des juxtapositions d'objets, de pensées, d'idées politiques, d'images, qui sont imperméables les unes aux autres. Ce sont comme des atomes qui ne peuvent pas s'interpénétrer. Un jour, nous trouverons le moyen de les connecter. Peut-être le plaisir de zapper est-il un exorcisme par rapport à cette impénétrabilité des choses : on se fabrique sa propre histoire à partir de morceaux épars... C'est le rêve d'une partition capable d'englober toutes ces informations disparates et indépendantes.

47 - Cela ne fausse-t-il pas la communication avec le public?

Georges Aperghis : Tout est difficile avec le public. Personnellement, je ne supporte pas les concerts, cet enchaînement de pièces différentes qui durent chacune queques minutes. Je pense qu'il faudrait changer cela complètement, mais je ne sais pas comment... Je peux imaginer quelque chose de plus intime, pour un petit public qui serait placé autour des instrumentistes. Je crois qu'en plus du plaisir d'être proche des musiciens, il y aurait une compréhension plus immédiate de la musique : on écouterait une œuvre, on pourrait en discuter... Il faudrait que les concerts soient autre chose qu'un concours du Conservatoire, que ce soit davantage une partie de plaisir et non pas une juxtaposition de pièces. En ce qui concerne le théâtre musical, je ne vois pas de rupture avec le public. Et puis, il ne faut pas attendre que le public suive toutes nos folies! Il faut bien qu'il ait un peu de retard, sinon à quoi servirait-on?

Nous sommes arrivés à un point où il existe trop d'informations. Et c'est là que Cage a été prophétique. Mes idées lui doivent beaucoup. Il est celui qui va contre cette folie de l'information. J'écoutais l'autre jour son Quatuor à cordes : c'est une musique qui semble appartenir à un siècle qui n'existe pas, avec des instrumentistes étranges ; cela me fait 
penser à des fausses reproductions de Duchamp. Il y a un très grand calme, une extraordinaire attention au silence, à chaque son.

- Cage a exercé une grande influence sur votre travail ?

51 Georges Aperghis: J'ai un énorme respect pour lui. L'exemple de Cage a été déterminant pour moi au milieu des années soixante. C'est grâce à Cage que j'ai pu voir la totalité de ce qui se faisait en Occident avec du recul, et avec de l'humour aussi ! Lorsque je suis venu à Paris, en 1963, je ne connaissais rien, à part quelques œuvres de Stravinsky, et le livre de Boulez : Penser la musique aujourd'hui ; mais c'était abstrait pour moi, parce que je ne connaissais pas la musique à laquelle ce livre était lié. Cage a été un choc. Je me souviens aussi d'avoir été très marqué par une pièce de Kagel, Sur scène, qui avait été donnée au "Domaine". Il y a toujours chez Kagel une tragédie traitée sur un mode sarcastique, ironique, et cela me touche beaucoup. Je pense dans une certaine mesure à Beckett. Chez l'un et chez l'autre, ce qui m'intéresse, c'est moins la forme que les personnes. Chez Beckett, derrière une phrase banale, il y a toujours un drame. Chez Kagel, quoi qu'il fasse, le drame est aussi présent, et c'est une vérité essentielle de son œuvre. 


\section{Quatre aspects du matériau musical et de l'écoute}

\section{Helmut Lachenmann}

Traduction : Jean-Louis Leleu

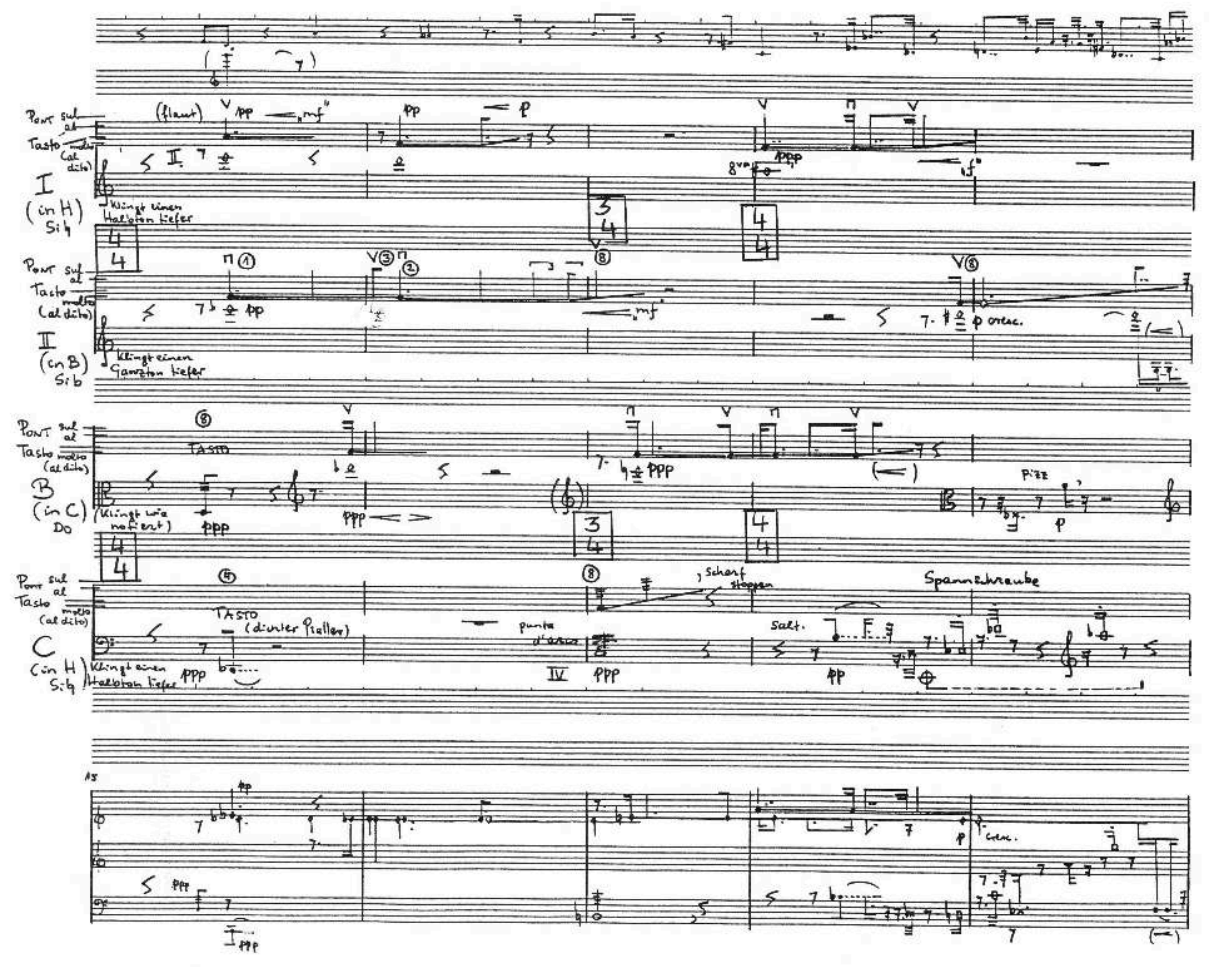

Un exposé que j'ai fait à Stuttgart en 1971 se terminait par la phrase suivante: "L'écoute est désarmée sans le secours de la pensée." Cette phrase, malgré son allure énergique, s'exposait, au fond, à toutes les critiques, et sans doute a-t-elle été la victime de sa propre vérité : une écoute désarmée - contre quoi ? et que signifie "pensée" ? Estce au cerveau ou au cœur que s'adresse la musique? 
2 Cette dernière question est traîtresse : penser n'empêche de sentir que lorsque ces deux facultés sont sous-développées. L'écoute est également désarmée sans le secours du sentiment. Mais il n'existe pas seulement un sentiment vide de pensée et une pensée vide de sentiment: plus grave encore, et fort répandue, est l'existence d'un "penser" vide de pensée et d'un "sentir" vide de sentiment. La pensée et le sentiment mis en branle par la musique tournent, en fait, autour de nos désirs et de nos besoins les plus profonds: notre soif de bonheur, de connaissance, de plénitude. L'industrie de la musique de variétés vit de la manière dont elle sait tromper nos désirs, tromperie qui ainsi s'étend également à nos sentiments et à nos pensées. L'institution culturelle des "philharmonies", par le faux usage qu'elle fait de notre tradition, prend à cette imposture une part fatale.

3 La musique contemporaine, que la mission historique dont elle s'est vue chargée au plus tard chez Schoenberg condamne à être au sein de la culture officielle un inévitable corps étranger, a sûrement souffert davantage de sa situation que le public qu'elle recherche justement en se détournant de lui. Cette manière de "se détourner de", dans la mesure où elle visait des formules et des formes devenues creuses, a été, depuis Schoenberg, la seule façon plausible de se tourner vers, au nom de ces désirs de l'homme dont je viens de parler. L'auditeur qui réfléchit l'a bien compris. Il ne se laissera pas non plus abuser par les proclamations claironnantes selon lesquelles (pour reprendre l'expression d'un commentaire de programme) l'"ère glaciaire" que nous a fait traverser pendant trente ans une avant-garde pleine d'arrogance est enfin terminée quand bien même l'on ne saurait mieux expliquer la période de boue - conséquence inévitable d'une ère glaciaire - dans laquelle nous nous trouvons manifestement aujourd'hui.

4 La musique a pour terrain propre le champ de forces qui se constitue entre, d'un côté, notre intériorité, et de l'autre le monde social. Ce sont là deux instances de force inégale, et le monde social pénètre, domine et manipule de mille manières notre intériorité. Sous son influence, cette tromperie commerciale dont nos désirs sont l'objet devient notre propre fait: nous abusant nous-mêmes, nous sentons et nous pensons ce que nous devons sentir et penser.

5 Notre écoute est sans défense tant que nous ne sentons pas et que nous n'employons pas les forces qui, en retour, partent de notre intériorité et pénètrent le monde social, intervenant dans ses rouages. Faire prendre conscience du potentiel que constituent, dans l'art et en nous, ces forces-là : telle est la tâche de cette pensée grâce à laquelle l'écoute peut résister à la tutelle qu'on lui impose.

6 Ce monde établi qui est le nôtre - monde dans lequel l'intériorité est socialement prise en charge, la beauté socialement frappée d'interdit -, c'est à travers lui que l'art doit s'affirmer, pour le compte du Moi qui, lui, ne peut être pris en charge, et au nom d'une intériorité qui porte la marque d'une réalité plus profonde. Autrement dit : l'intériorité qui se communique musicalement doit se mesurer au monde social à l'aide des moyens que ce monde tient à sa disposition, au risque de provoquer des conflits là où la société attend que règne l'harmonie et que soient confirmées les valeurs qu'elle défend.

7 Si je me propose, dans les pages qui suivent, de mettre en lumière quatre traits fondamentaux de notre écoute, je ne ferai, par là même, qu'examiner le matériau musical sous quatre aspects élémentaires, et cet appel nécessairement intellectuel à notre expérience réelle, s'il peut indiquer à la pensée des directions possibles, ne peut 
en aucune façon la dispenser - pas plus que le sentiment - de l'effort créateur qui lui revient.

8 Avant de présenter quatre traits fondamentaux de notre écoute, je voudrais commencer par énoncer deux truismes :

9 1. Composer ne consiste pas à "mettre ensemble", mais à "mettre en relation" - c'est-àdire à modeler et à ordonner les moyens musicaux auxquels on fait appel pour en faire les supports de l'unité à réaliser, ces moyens acquérant, par là même, une fonction expressive. L'expression musicale n'existe que par la vertu de telles relations.

10 2. Le matériau musical est autre chose qu'une simple matière première docile qui attend uniquement que le compositeur la charge d'expression, et ainsi lui donne vie, au sein de tel ou tel ensemble de relations : il est déjà, lui-même, inscrit dans des relations et marqué expressivement, avant même que le compositeur ne s'approche de lui. Ces traits qui se sont gravés dans le matériau proviennent de la même réalité qui nous a marqués nous-mêmes, compositeurs et auditeurs - qui a marqué notre existence et notre conscience. Si la composition doit dépasser le stade de l'exploitation, purement tautologique, d'une expressivité déjà existante et habituelle, c'est-à-dire de l'exploitation confortable de ces relations qui sont données a priori dans le matériau et dans l'auditeur -, et si elle doit, en tant qu'acte créateur de la conscience, rappeler ce potentiel humain qui donne sa dignité à l'homme en tant que créature capable de connaître et de muer cette connaissance en action - bref : si l'acte de composer doit avoir une portée artistique, composer, dans ce cas, ne fait pas que consister à "mettre ensemble", mais signifie toujours "se mesurer" avec ces relations qui conditionnent le matériau et l'écoute - c'est-à-dire : à partir des lois connues auxquelles le matériau est assujetti, tirer, à l'aide de la raison et de l'imagination individuelles, de nouvelles lois et de nouveaux principes formels, soumettre ainsi le matériau à un nouveau contrôle, à une nouvelle exploration, à un nouvel éclairage, et faire que ces relations données a priori soient, tout à la fois, niées et conservées. De quelque manière que l'on procède ici, rationnellement ou intuitivement, on ne peut s'acquitter par aucune autre voie de cette tâche que Schoenberg a formulée mieux que nul autre: "Le but le plus haut de l'artiste : s'exprimer."

11 Des quatre aspects sous lesquels peut être envisagé le matériau, le premier qui vient à l'esprit est celui de la tonalité. Dans la mesure où il est à la base de notre tradition musicale et de nos conventions esthétiques, cet aspect joue, dans notre vie musicale quotidienne, un rôle prépondérant. La tonalité : ce terme recouvre l'ensemble des expériences que nous faisons avec le dispositif esthétique légué par la tradition, avec ses catégories d'harmonie, de mélodie, de rythme assujetti à une métrique, de cadence le mot étant pris ici dans son acception la plus large-, à quoi s'ajoutent les techniques d'écriture et les principes formels découlant des dites catégories ; mais il recouvre aussi les différentes pratiques liées à ce dispositif - pratique instrumentale, notation, interprétation -, car il n'y a pas que le matériau qui soit préformé tonalement: notre propre conscience, notre propre écoute le sont dans une égale mesure.

12 Je ne puis rendre compte ici des implications esthétiques et historiques de cette conquête occidentale qu'est la tonalité : une magie de la vibration harmonique en quelque sorte imprégnée de rationalité, et qui, même percée à jour, demeure encore intacte. Je voudrais seulement attirer l'attention sur deux aspects de la tonalité : 
13 - d'une part : si émoussée qu'elle puisse être, l'expérience de la tonalité, dans la mesure où elle a pour contenu l'identification à soi et à la société, est une expérience au sens plein, et c'est ce qui lui donne une vitalité et une capacité de survie terrifiantes.

14 - d'autre part: la notion de "tonalité" ne s'applique pas à celui qui s'installe passivement dans cet état d'identification, mais bien à celui qui, tout en quittant cet état, n'en continue pas moins de se référer à lui, et pour qui la tension ainsi créée prend une dimension expressive - c'est-à-dire esthétique. La tonalité est ainsi définie par une dialectique toujours précaire - on l'a vu depuis - de la consonance et de la dissonance, ce qui permet et commande de rattacher au principe tonal toute expérience musicale fût-ce la plus insolite - en tant qu'expérience de la dissonance, dont le coefficient de tension augmente encore à mesure que l'on s'éloigne - peu importe de quelle manière du centre tonal. Autrement dit: il n'y a rien dont on ne puisse rendre compte, et conséquemment tirer parti, à l'aide des catégories de la tonalité.

15 Quelque volonté que l'on ait de s'éloigner de la tonalité, celle-ci vous rattrape toujours. Toutefois, le problème n'est pas non plus de savoir comment échapper à l'emprise tonale, ou par quels biais s'en accommoder de nouveau: il s'agit bien plutôt de reconsidérer ces aspects tonaux par rapport à un contexte général en constante évolution.

16 L'un des éléments de cette évolution est l'importance prise par un autre aspect du matériau et de l'écoute: celui qui se rapporte à la réalité physique immédiate du phénomène sonore, et donc commence avec les propriétés acoustiques perceptibles. Cet aspect s'est fait jour au cours de la dissolution progressive des catégories tonales opérée au plus tard par Webern, et après lui par les compositeurs sériels, chez qui le contrôle du matériau, issu à l'origine de la technique classique de la variation et du développement, s'est finalement appliqué, de façon radicale, à toutes les propriétés acoustiques contrôlables du matériau : ce qu'on a appelé ses "paramètres".

17 Ce n'est pas que ces derniers, auparavant, soient restés incontrôlés; mais leur rôle de quasi-véhicules d'une pratique axée sur la tonalité (de simples "récipients", remplaçables, du langage tonal) les reléguait au second plan, ou souvent à l'arrièreplan, comme en témoigne, par exemple, une discipline apparemment séparable de l'écriture tonale : l'orchestration.

18 Le premier stade de la mise au jour de cet aspect du matériau a été la "pensée en paramètres" des compositeurs sériels. A l'occasion de recherches sur la relation sonforme, j'ai proposé, dans les années 60 , une typologie qui rend compte plus précisément de cet aspect, dans la mesure où elle envisage le son comme le produit, non plus de son organisation microtemporelle (c'est-à-dire de rapports d'ondes internes), mais de son organisation rythmique externe - macrotemporelle -, c'est-à-dire à la fois comme état et comme processus, et où elle établit une relation directe entre la notion de "son" dans le sens où l'on se sert de la pression acoustique pour articuler le temps - et celle de "forme", qui obéit à la même définition.

19 C'est ainsi que ce qu'on appelle un "Kadenzklang"'.

20 (Par exemple, une attaque suivie d'une résonance naturelle ou artificielle, c'est-à-dire écrite : un coup de tam-tam, ou un accent de piano prolongé par un accord de cuivres diminuendo) serait en même temps une "cadence sonore" (Klangkadenz), soit une pente d'intensité ou d'énergie perceptible en tant que forme ; de même, un "Farbklang" - par exemple, un accord d'orgue ou un accord de vents tenus - serait en même temps une 
"couleur sonore" (Klangfarbe), à savoir la perception caractéristique d'un temps statique, dont la durée resterait à déterminer ;

21 - ou encore : un "Fluktuationsklang" - par exemple, une figure en arpège indéfiniment répétée comme on en rencontre dans la musique impressionniste ou dans la musique baroque, ou même un simple trille - pourrait en même temps être décrit formellement comme une "fluctuation sonore" (Klangfluktuation), à savoir une expérience statique du temps formée de mouvements périodiques, d'une durée à déterminer ;

22 - de même, un "Texturklang" - par exemple, le bruit de la circulation sur une place publique - pourrait être décrit formellement comme "texture sonore" (Klangtextur), à savoir une expérience statique du temps formée d'une multiplicité d'événements hétérogènes isolés, produisant une impression globale statistique : une sorte de chaos ayant son caractère propre ;

23 - enfin (mais je renverse ici la formulation) : une "structure sonore" (Klangstruktur) autrement dit une forme - pourrait être saisie en même temps comme un "son structurel" (Strukturklang) typique, c'est-à-dire, non pas un chaos, mais au contraire un ordre ayant son caractère propre - un ordre formé de composantes sonores hétérogènes, produisant un champ de relations complexe pensé dans tous ses détails, et de la combinaison desquelles résulte, non pas simplement une impression statistique, mais une expression ciselée dans ses moindres détails, et dont la présence, cette fois, est intemporelle. Par exemple : une "Bagatelle" de Webern, une fugue de Bach, ou toute œuvre, quelle qu'elle soit, formant un tout cohérent.

24 Je ne puis m'étendre ici sur les différentes formes de combinaison et d'interaction de ces cinq types sonores. Il convient cependant d'indiquer, au moins, les liens étroits existant entre ce qu'on sépare si volontiers, de manière dualiste, comme étant d'un côté la nature, et de l'autre l'esprit :

- alors que, dans le cas du "Kadenzklang" on pourrait encore parler d'une pente naturelle

- la secousse d'énergie initiale, en tant que réflexe mécanique plus ou moins contingent, ne disant rien de plus, dans ce cas, sur son origine-, on peut déjà se demander, s'agissant du "Farbklang" et du "Fluktuationsklang", quelle sorte de nature peut se cacher là derrière : la nature, même la nature humaine, ne fournit pas d'elle-même si facilement de telles figures acoustiques. La répétition périodique régulière d'un processus vibratoire suppose déjà l'action d'une forme d'énergie moins primaire : à savoir la volonté d'une discipline précise dans l'émission vocale ou instrumentale, ou dans l'énoncé d'une formule rythmique.

- Les textures, elles, sont déjà davantage fournies spontanément par la nature. Les énergies qui sont alors à l'œuvre - même si elles sont hautement organisées - apparaissent comme les victimes d'un principe en quelque sorte aveugle, qui ignore tout de ce qu'il provoque: les autos ne circulent pas en vue de produire ensemble une texture sonore-mais elles ne la produisent que de cette façon.

- Enfin, chacune des formes d'énergie qui, dans le cas du "son structurel", opèrent la synthèse des différentes composantes au sein d'une entité sonore organisée renvoie à, et porte l'empreinte d'une volonté dont l'action est chargée de sens - c'est-à-dire de l'esprit. Saisir par l'écoute une structure, en lire les détails à la lumière de l'ensemble, et leur donner ainsi une nouvelle intensité : tout cela implique une mobilisation créatrice de l'esprit, qui n'est autre que le dernier degré de complexité de la nature.

Et si, en guise de refrain à propos de ce second aspect du matériau et de l'écoute, je dis en résumé qu'il n'existe aucun matériau qui ne se laisse décrire comme un phénomène purement physique - c'est-à-dire comme une forme d'organisation plus ou moins 
complexe du microtemps et du macrotemps -, ce "plus ou moins complexe" inclut tout l'éventail qui va du son naturel, simple ou recréé, à la "forme-son" médiatisée dialectiquement, autrement dit : à l'expérience de la structure comme manifestation de ce produit naturel hautement cultivé que nous nommons "esprit humain".

Dans notre tentative de classer, par ordre de complexité croissante, les catégories de la perception sensible proprement dite, nous en sommes arrivés, avec ce dernier type celui du "son structurel" ou de la "structure sonore" - au troisième de ces aspects fondamentaux du matériau qui forment le thème du présent exposé : aspect qui ne se laisse pas définir simplement comme l'organisation pleine de sens d'éléments pouvant être décrits en simples termes d'acoustique - comme on pourrait le croire tant qu'on ne voit dans la "structure" qu'un ultime avatar du son perçu dans sa seule réalité physique. La notion de "structure" définit bien plutôt un nouvel aspect fondamental du matériau et de l'écoute. Saisir le matériau comme structure, c'est le saisir comme le fruit d'une intervention consciente de l'intérieur d'un ordre préexistant. Cette notion de "structure" représente ainsi, à côté de l'histoire, des conventions et de la nature, la véritable instance spirituelle et poétique, et elle appelle l'attention sur cet acte de la volonté qui intervient de manière nécessaire dans la matière, et y instaure un ordre dans l'exacte mesure où il nie tel ordre qui y régnait au départ, et où, mettant en question un langage péexistant et habituel (par exemple, mais pas seulement, le langage tonal), il le soumet à un nouvel éclairage et à une nouvelle appréciation, le complète, l'explore - le métamorphose.

La notion de "structure" ne repose donc pas seulement sur des catégories d'ordre, mais également sur l'expérience de la négation. Et il n'existe aucun matériau - voici de nouveau mon refrain-, pas même le matériau le plus simple et le plus homogène, où cet aspect de la structure ne se présente à nous dans sa dialectique d'offre et de refus c'est-à-dire où des éléments hétérogènes, d'origine différente et appartenant à des univers différents, n'entrent dans une nouvelle combinaison, n'abandonnent, ce faisant, les liens qui les rattachaient à leur univers, et en conservent, néanmoins, la trace négative : la table, perçue comme structure, est un arbre qu'on a détruit, quelque chose dont la nature a été privée, et l'univers des hommes enrichi.

Cette intervention dans des structures ou des réseaux préexistants - peu importe ce sur quoi elle s'effectue consciemment - ne se limite pas, naturellement, à tel ou tel ordre musical préexistant (par exemple, l'ordre tonal) : elle s'effectue également - que cela soit conscient ou non - sur l'ensemble des effets et des associations qui se rattachent du dehors - c'est-à-dire par le biais de la réalité extramusicale - au matériau utilisé. Car chaque matériau est marqué de façon décisive par le rôle qu'il joue dans l'existence de l'homme; il se charge ainsi d'associations, qui définissent son "aura" - c'est-à-dire la manière particulière dont il nous est familier. Nous en arrivons ainsi au dernier aspect fondamental du matériau et de l'écoute. L'aura que l'écriture confère à la musique, du fait même de l'intervention dont la structure est le produit, perd toujours pour nous, en même temps, son caractère familier, et parfois même devient méconnaissable. Et c'est précisément cette forme de distance, sans laquelle aucun rapport créateur au matériau n'est possible, qui, lorsqu'elle n'est plus ressentie, spontanément, que comme une privation d'identité, est à l'origine du conflit qui s'installe entre la musique contemporaine et le public. Les compositeurs sériels, dans la mesure où leurs conceptions structuralistes ne tenaient aucun compte de l'aura du matériau qu'ils utilisaient - de notre rapport de 
familiarité avec ce matériau-, ont souvent, précisément lorsqu'ils visaient un nouvel ordre, mis sur pied en réalité un chaos expressif, et, dans de nombreuses œuvres, un méthodique amas de décombres. Que vient faire la cloche de vache, sur laquelle Mahler comptait encore pour apporter dans sa musique une bouffée d'air pur, bien loin du tumulte du monde, que vient faire cet objet sorti de la vie quotidienne des campagnes, dans une œuvre comme Gruppen de Stockhausen, entre ce joujou de salon qu'est le célesta, et ce vénérable instrument du Jugement dernier, à la fois guerrier et religieux, qu'est le trombone? La réponse paraît absurde: les trois instruments forment ensemble des "spectres formels", c'est-à-dire : du contrepoint des trois instruments résultent des figures certes hiérarchisées, mais présentant des caractéristiques de figuration similaires. Rien à dire contre l'idée de "structure sonore" à l'œuvre dans cette composition, mais quelle violence faite au caractère idyllique que les cloches de vaches nous avaient rendu si familier. Au reste, le sacrifice de cette aura est certainement justifié, car les Gruppen représentent justement, du point de vue du message artistique, une expérience nouvelle décisive, et le son de ces cloches devenues méconnaissables témoigne, lui aussi, d'une nouvelle aura: celle qui caractérise la sonorité orchestrale de Stockhausen. Néanmoins : se borner à nier l'agressivité dont cette musique fait preuve envers l'auditeur bourgeois, ou pis encore, vouloir conférer à cette agressivité un caractère mystique, au lieu de le ramener à ses causes réelles, à savoir le rapport négatif du compositeur à ce qui était jusqu'alors familier, serait rester sourd à ce dernier aspect du matériau, celui de l'aura, en tant que véhicule d'expériences courantes de la réalité vécue : celles qui relèvent de la vie quotidienne, des différentes couches sociales, de la sphère religieuse, de la (ou des) cultures(s), de la technique, de l'histoire, des paysages, des classes, peut-être aussi du subconscient, du monde onirique, etc. Cet aspect de l'aura me paraît être le complément décisif et le correctif essentiel de la volonté d'autonomie propre à la pensée structurale.

31 La relation qui s'établit entre la structure écrite et l'aura propre au matériau, la manière dont la structure - loin de la nier simplement - tient compte de cette aura, en absorbe l'expressivité, et même tire de sa confrontation avec elle l'impulsion créatrice autrement dit: la manière dont la notion de "structure" sacrifie à cet aspect de l'“aura", ou adapte à lui sa volonté d'autonomie, son côté autarcique et sa complexité foncière, quitte à simplifier ses règles en conséquence, c'est à elle que se reconnaît l'originalité d'un projet compositionnel.

Tonalité-réalité physique-structure-aura : il n'est pas de musique qui ne soit accessible à tout auditeur, de manière directe et intuitive, dans le jeu de ces quatre aspects. Il ne reste plus rien à décrypter. Et pourtant : un examen attentif des propriétés du matériau ne peut jamais conduire, par lui-même, à des interprétations uniques, et encore moins à des conclusions techniques ou esthétiques - et à des doctrines - uniques. Car les relations qui sont à l'œuvre dans le matériau ne sont jamais univoques; chacune d'elles est inscrite elle-même dans d'autres relations, est elle-même un conglomérat de relations - conscientes ou inconscientes, réfléchies ou irréfléchies, rationnelles ou irrationnelles -, et le compositeur n'a pas moins de difficultés que tout autre à comprendre sa musique. On pourrait penser, dans ces conditions, que le compositeur qui s'était prêté aux réflexions développées ci-dessus, ainsi que l'auditeur qui réfléchit, se retrouvent finalement les mains vides. Si loin qu'il aille dans son travail, le compositeur ne saisit et ne contrôle jamais qu'un simple coin du tout: ce dernier lui échappe, et reste, en dernier ressort, insaisissable. Qui sait? Peut-être est-ce le 
compositeur lui-même qui, semblable à la queue qui frétille avec le chien, est contrôlé par le tout.

Il n'y a là aucune résignation, mais bien plutôt la confirmation qu'une telle réflexion est juste, puisqu'elle prive le compositeur - comme l'auditeur - de toute recette. Il ne lui reste qu'une volonté d'expression qui se trouve à travers un enfer d'incertitudes, une faculté de penser et de sentir plus aiguë dans son rapport avec le matériau, et, dans cette confrontation, un instinct parfaitement éveillé : réflexes de la queue dans l'espoir que le chien frétille avec elle. Ou pour exprimer cela plus solennellement, avec les mots de Mahler: je ne compose pas, je suis composé (ou pour l'auditeur: je ne comprends pas, je suis compris).

Ma dernière remarque s'appliquera, une fois encore, à la notion de "structure", dont le modèle - celui d'une polyphonie d'agencements - était jadis apparu comme le moyen de triompher - et avait effectivement permis de triompher - de la vieille emprise tonale et des tabous qu'elle entraînait. On n'a pas cessé, dans les dernières années, de porter aux nues la pensée structurale, et plus souvent encore de pester contre elle. Et il est indubitable que ce modèle a, dans une large mesure, dégénéré en recette, et qu'on en a fait un usage académique. La notion de structure demande elle-même à être perpétuellement remise en question. Je ne crois pas, pour ma part, qu'on puisse se passer de penser en termes de structure. Mais il est nécessaire qu'une telle pensée, et que les techniques structurales elles-mêmes, soient sans cesse remises en cause en se trouvant confrontées à la réalité, c'est-à-dire aux autres aspects réels du matériau - il est nécessaire qu'elles se perdent, se retrouvent et se redéfinissent. La musique n'a de sens que dans la mesure où, par-delà sa propre structure, elle renvoie à des structures, à des réseaux - autrement dit à des réalités et des potentialités présentes autour de nous et en nous-mêmes.

Quatre aspects fondamentaux du matériau musical et de l'écoute

\begin{tabular}{|c|c|c|c|c|}
\hline Sphère d'action & Aspect fondamental & Eléments actifs & Champ de tensions & Situation expressive \\
\hline $\begin{array}{l}\text { I. Histoire } \\
\text { Convention } \\
\text { Société }\end{array}$ & TONALITÉ & $\begin{array}{l}\text { Harmonie, mélodie, } \\
\text { rythme, cadence, } \\
\text { techniques d'écriture } \\
\text { tonales, formes, } \\
\text { pratique musicale }\end{array}$ & $\begin{array}{l}\text { Consonance/ } \\
\text { Dissonance } \\
\text { (tonal/atonal) }\end{array}$ & $\begin{array}{l}\text { Geste au sens plein } \\
\text { (magique/rationnel) }\end{array}$ \\
\hline $\begin{array}{l}\text { II. Matière } \\
\text { (nature et formes } \\
\text { d'organisation } \\
\text { tirées de celle-ci, } \\
\text { esprit) }\end{array}$ & $\begin{array}{l}\text { RÉALITÉ } \\
\text { PHYSIQUE } \\
\text { (perception sensible } \\
\text { proprement dite) }\end{array}$ & $\begin{array}{l}\text { Paramètres, } \\
\text { "Types de son": } \\
\text { (Kadenzklang - } \\
\text { Klangkadenz } \\
\text { Farbklang -Klangfarbe } \\
\text { Fluktuationsklang - } \\
\text { Klangfluktuation } \\
\text { Texturklang-Klangtextur } \\
\text { Strukturklang - } \\
\text { Klangstruktur) }\end{array}$ & $\begin{array}{l}\text { Son/Forme } \\
\text { (microtemps/ } \\
\text { macrotemps) }\end{array}$ & $\begin{array}{l}\text { L'expérience } \\
\text { "libérée" du } \\
\text { temps-son }\end{array}$ \\
\hline $\begin{array}{l}\text { III. Esprit } \\
\text { Volonté } \\
\text { Imagination }\end{array}$ & STRUCTURE & $\begin{array}{l}\text { Principes d'organisation } \\
\text { (règles, lois) }\end{array}$ & $\begin{array}{l}\text { Détail/Tout } \\
\text { organisation// } \\
\text { désorganisation }\end{array}$ & $\begin{array}{l}\text { Offre-refus } \\
\text { individuation } \\
\text { expression }\end{array}$ \\
\hline $\begin{array}{l}\text { IV. Existence } \\
\text { Réalité } \\
\text { Souvenir }\end{array}$ & AURA & $\begin{array}{l}\text { Expériences vécues } \\
\text { (signaux de la vie } \\
\text { quotidienne, technique, } \\
\text { nature, religion, cultures(s), } \\
\text { subconscient, passé, etc.) }\end{array}$ & $\begin{array}{l}\text { Expérience esthétique } \\
\text { Réalité } \\
\text { Conscience/Existence, } \\
\text { Sensation/Expérience }\end{array}$ & $\begin{array}{l}\text { Mise à distance d'une } \\
\text { réalité familière }\end{array}$ \\
\hline
\end{tabular}




\section{NOTES}

1. Les expressions reprises et renversées ici par Lachenmann n'ont pas d'équivalent direct en français. La traduction de "Kadenzklang" par "percussion-résonance”, par exemple, ôterait tout sens au passage. Aussi avons-nous préféré garder les expressions allemandes, les exemples indiquant clairement, dans chaque cas, de quel type de son il est question (N.D.T.). 


\section{L'erreur comme nécessité}

\section{Luigi Nono}

1 Le silence.

2 Il est très difficile à écouter.

3 Il est très difficile d'écouter, dans le silence, les autres. D'autres pensées, d'autres bruits, d'autres sonorités, d'autres idées. Par l'écoute, on essaie souvent de retrouver soimême chez les autres. On veut retrouver ses propres mécanismes, son propre système, son rationalisme - chez l'autre. Il y a là une violence tout à fait conservatrice.

5 Au lieu d'écouter le silence, d'écouter les autres, on espère s'écouter encore une fois soi-même. Cette répétition est académique, conservatrice, réactionnaire. C'est un mur dressé contre les pensées, contre ce qu'il n'est pas possible, aujourd'hui encore, d'expliquer. C'est le fait d'une mentalité systématique, basée sur les a priori - intérieurs ou extérieurs, sociaux ou esthétiques. On aime le confort, la répétition, les mythes ; on aime écouter toujours la même chose, avec ces petites différences qui permettent de démontrer son intelligence.

6 Ecouter la musique.

7 Cela est très difficile.

8 Je crois qu'aujourd'hui, c'est un phénomène rare.

9 On écoute quelque chose de littéraire, on écoute ce qui a été écrit, on s'écoute soimême...

10 L'espace.

11 La salle de concert traditionnelle est un espace horrible, car elle ne donne pas des possibilités, mais une possibilité.

12 Il y a pour chaque salle un travail spécifique à faire, comme autrefois on écrivait pour tel ou tel lieu, telle ou telle circonstance. La musique que je cherche actuellement est écrite avec l'espace: elle n'est jamais semblable dans n'importe quel espace, mais travaille avec celui-ci. Cela permet une grande diversité. 
Pour Musil, s'il existe un sens de la réalité, il doit aussi exister un sens des probabilités. Ce que l'on a choisi n'est pas forcément juste ; peut-être, ce qui n'a pas été choisi était-il plus juste. On passe par une telle expérience dans le travail en studio : il y a beaucoup d'imprévus, de hasards, d'erreurs - mais des erreurs qui ont une grande importance, comme Wittgenstein l'a théorisé.

14 Car l'erreur casse les règles. Elle est transgression. Elle est opposition à l'institution stabilisée. Elle est ce qui pousse vers d'autres espaces, d'autres cieux, d'autres sentiments humains - à l'intérieur et à l'extérieur, sans dichotomie entre les deux, contrairement à ce que la mentalité banale et manichéiste soutient encore aujourd'hui.

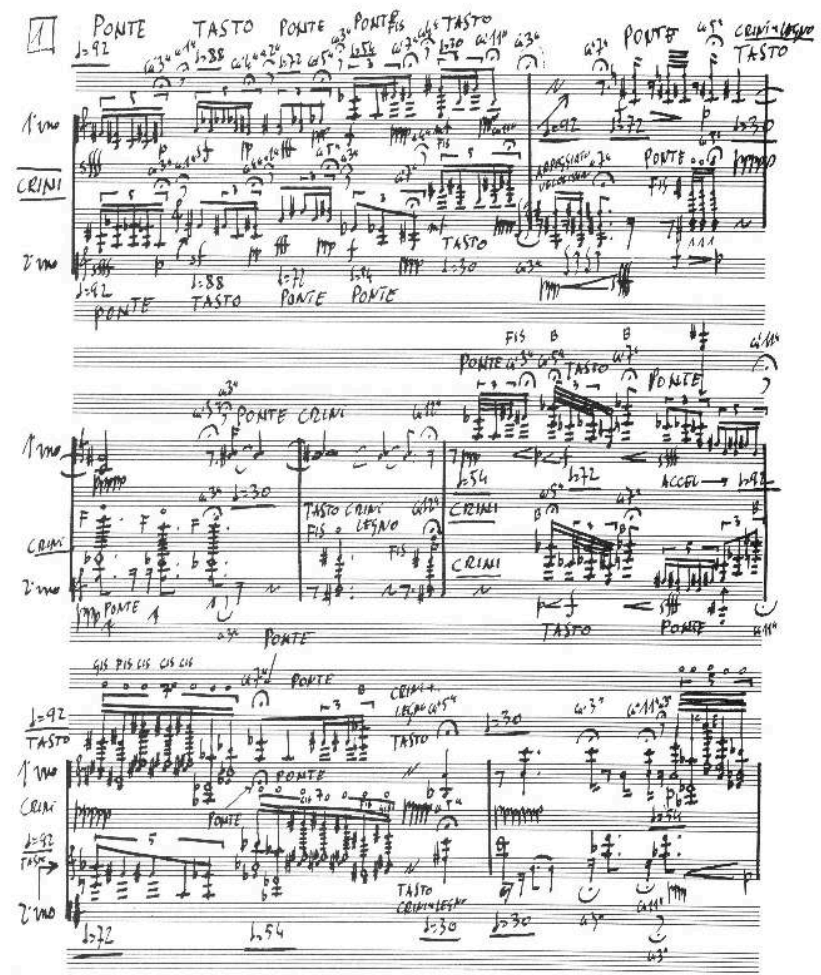

15 Diversité de la pensée musicale : non des formules, des règles, ou des jeux. Mais une pensée qui transforme la pensées des musiciens, plutôt que de leur donner un métier permettant de faire de la musique soi-disant actuelle. Schoenberg, lorsqu'il organisait ses concerts, imposait toujours de très nombreuses répétitions : pour la Symphonie de chambre opus 9, il en fit une dizaine; mais il ne fit pas de concert. Cela m'a fait réfléchir : le travail de recherche est infini. La finalité, la réalisation, appartiennent à une autre mentalité. Peut-être l'idée de Schoenberg n'est-elle pas une folie et contientelle une grande vérité. Ainsi, dans le travail de recherche ou de répétition, il éclate souvent des conflits. Ce sont des moments très émouvants. Vient ensuite la ritualité du concert. Mais peut-être est-il possible de changer cette ritualité et de réveiller l'oreille. Réveiller l'oreille, les yeux, la pensée, l'intelligence, le maximum d'intériorisation extériorisée : voilà l'essentiel aujourd'hui. 


\section{Le nom de la liberté}

\section{Mauricio Kagel}

Traduction : Anne Lavanchy

\section{LES IDEES FIXES}

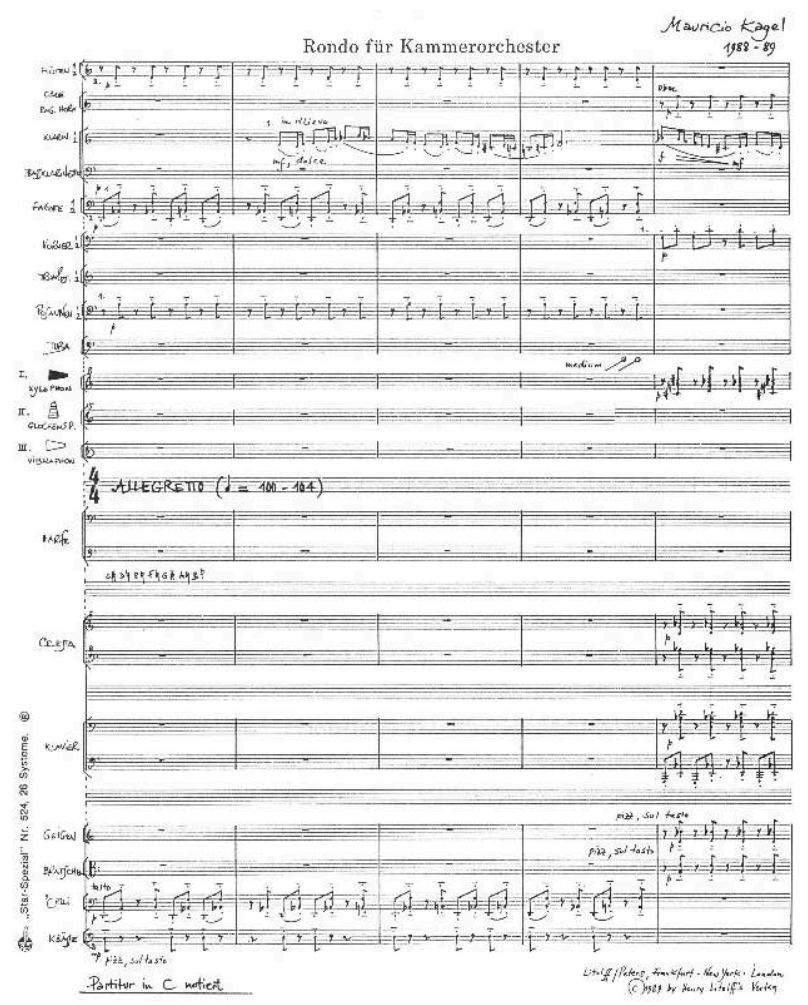

C'est étrange, mais vrai ; en refléchissant au sujet de cette manifestation, je ne pouvais m'empêcher d'en modifier inconsciemment le titre. J'ai dû finalement accepter que Le nom de la liberté se transforme et devienne Au nom de la liberté. Pour ce léger changement, lourd de conséquences, je vous demande à tous pardon. Pourtant cette démarche me semble logique: que de faits et de méfaits sont aujourd'hui encore accomplis au nom de cette liberté, tout comme "au nom du peuple", "au nom de la 
justice" ou, et ceci est le comble de la prétention, "au nom de Dieu", des formules aussi lapidaires que pathétiques.

2 Mais peut-être ne peut-on définir la liberté, ou mieux encore, ce que nous nommons liberté, que par des détours. Comme la capacité de notre mémoire se trouve dépassée lorsqu'on dit "depuis toujours" pour justifier des coutumes, des comportements, de la même façon la mémoire de l'homo erectus est trop faible, trop floue et - probablement aussi par légitime défense - trop friable dès qu'on y cherche un appui infaillible. Et c'est précisément à cause de l'incapacité de l'homme à transférer son expérience d'un domaine à un autre selon les lois de la raison, que se révèlent les différences et les défaillances de notre conception si précieuse de la "liberté".

3 Tout d'abord, il faut accepter que la liberté ne puisse provenir que de la somme de plusieurs libertés distinctes. Ainsi l'on sera forcé de mettre un frein à cette tendance, innée dès qu'il s'agit de l'essentiel, à l'idéalisation. Car toutes ces libertés distinctes aspirent - même si elles paraissent peu importantes - à être nommées. Elles exigent des solutions, des chemins et des moyens pour transformer éventuellement leur existence factice en liberté concrète. Celui qui, comme moi, a vécu le dernier avènement, pour l'instant, du concept de liberté dans le domaine de la musique après 1950, ne pourra pas s'empêcher de comparer les différentes manières dont on utilise de tels mots clés et d'en dégager des similitudes et des contradictions. Et c'est là qu'apparaissent les difficultés. Ce qui, pour certains, était liberté, n'était pour d'autres que l'indice d'un chaos dangereux. Alors que beaucoup de compositeurs considéraient cette musique comme le bonheur d'une invention enfin libérée, ils étaient encore plus nombreux, interprètes, critiques et auditeurs, qui la ressentaient comme une nouvelle manifestation catastrophique de la modernité. Peut-être est-ce justement là un des aspects caractérisant la liberté : elle dépend avant tout du point de vue de l'observateur. La musique, l'art, la littérature et les médias souffrent considérablement de cette relativité dès qu'ils commencent à toucher au concept traditionnel et institué de liberté. Mais peut-on vraiment "toucher" aux libertés codifiées de la parole, de la pensée, de l'action? On pourrait croire que les limites de la liberté sont si précises, que tout ce qui ne coïncide pas avec elle peut être considéré comme un symptôme de maladie. Et pourtant, il n'en est rien. C'est vrai que l'absence de liberté est contagieuse, au point que cela nous fait souvent peur - et nous rend muets, parfois même nous pousse à la résignation et à la léthargie. En contrepartie, la liberté est peu spectaculaire car elle n'est en équilibre que lorsqu'elle est un état normal. Il n'y a pas de syndrome de la liberté qui aurait besoin d'une quelconque thérapie. On ne remarque la liberté que lorsque son absence est évidente.

4 Et pourtant, de la même manière que l'intelligence connaît des limites, la bêtise peut être illimitée. La liberté est un concept hautement relatif dont la théorie prospère lorsque la réalité est instable. Cela est aussi vrai pour la vie politique que dans le domaine de l'art. Bien que l'art ne veuille pas toujours être politique, il peut le devenir nolens volens si les circonstances exercent une pression idéologique. On est enclin à comparer les coulisses peu stables de la liberté à un décor en carton-pâte : derrière, il n'y a rien. Sauf parfois l'équation précise d'une utopie parfaite: ici l'être, là-bas la liberté. "Etre". "Libre". Etre libre, une minuscule sortie de service dans un décor rigoureusement construit? Prenons l'exemple de la musique. Le renouveau perpétuel est partie inhérente de la composition. Tout ce que le compositeur hérite du passé n'est qu'une part de lui-même, une sorte de présent à effet rétroactif. La scission entre hier 
et aujourd'hui n'est pas une réalité pour ceux qui appartiennent à ma corporation, car les différences d'un langage musical à l'autre sont moins significatives et plus changeantes que les mesures que nous avons établies pour le concept de qualité. Cela mène inéluctablement à des conflits: ce qui, pour certains, va de soi, sera incompréhensible pour d'autres.

5 Notre rapport avec la musique est basé sur la fidélité au texte bien que même celle-ci ait été l'objet de nombreuses interprétations. Au fil des siècles, les compositeurs et les interprètes ont essayé ensemble d'affiner, de rendre plus efficaces la notation et l'instrumentation. Et pourtant ces dernières années ont vu naître un courant diamétralement opposé à celui-ci, renouvelant totalement l'interprétation. Il s'agissait de gagner une dimension supplémentaire, celle de la multiplicité de l'interprétation et de l'écriture musicale. Qu'à travers les surprises ainsi ménagées, on espérât atteindre une partie de liberté, on ne peut le nier. La locution latine ad libitum a été citée certainement souvent et trop facilement. Mais l'obligation d'entretenir un rapport libéré avec ce que nous appelions, à tort, la "liberté", a subjugué la plupart d'entre nous. Il s'agissait alors de proposer plusieurs possibilités qui encourageraient l'interprète à donner une version unique et personnelle de l'œuvre. Les dés tombaient de manière méthodique à cette époque et le "Alea jacta est" de Jules César a été démontré sur le plan compositionel. Ce faisant, on ne réalisait pas que ce n'est qu'au moment où César a passé le Rubicon et dit son mot historique que la guerre civile a commencé pour de bon.

6 L'évolution de l'utilisation de formes spécifiques de la liberté dans l'interprétation s'est déroulée, ces dernières années, de manière moins violente mais plus instructive. Le nombre des musiciens qui s'y opposèrent fut légion. La douteuse ou - pour certainsextraordinaire opportunité de pouvoir participer à la composition a été presque unanimement refusée. Des agressions sans nombre et des malentendus sont survenus et nourrissent depuis lors des dissensions qui se déroulent toujours selon le même scénario. Quelques compositeurs le regrettent et en accusent la vie musicale, la société, l'éducation, l'état, l'Europe ou l'imperfection de ce monde. D'autres, et j'en suis, sont heureux que cette farce soit terminée pour l'instant, les débats n'ayant jamais été essentiels, ni pris au sérieux. Ce n'est pas en vain que l'ontologie différencie de manière tout à fait artificielle la connaissance et la réalité. De manière artificielle précisément, pas artistique. Beaucoup d'interprètes ne se sentent libres que lorsqu'ils savent leur partition par cœur et qu'ils la maîtrisent du point de vue technique. Le prix de cette liberté est un travail acharné et continu, mais personne ne devrait revenir à l'idée que le travail passe avant la liberté. C'est plutôt le contraire qui est juste, la liberté demande du travail ${ }^{1}$.

7 Il en va de même pour l'idéal d'une musique réellement libre et spontanée exprimée à travers une improvisation. C'est lorsque l'on cherche cette inspiration pure qui pourrait mener à la source de la musicalité créative que la réflexion, l'expérience et la discipline sont incontournables. On pourrait débattre du rapport entre la liberté et l'autodiscipline avant chaque discussion sur l'improvisation musicale et ce débat pourrait servir-pourquoi pas - d'exergue à l'improvisation planifiée qu'est notre existence. Peut-être la liberté n'est-elle qu'un fantôme nécessaire, semblable à cette éternelle carotte qui pend devant l'âne pour qu'il continue à avancer. Le nom de la liberté reste l'un des plus beaux, il n'empêche que le comportement de certains de ses défenseurs peut être douteux et contradictoire. Lorsque dans les années soixante-dix, 
la vie culturelle et les rédactions des feuilletons de la République fédérale allemande furent envahis par des commissaires politiques autoritaires et élus par eux-mêmes, nous étions nombreux à penser à l'époque de l'Inquisition et aux modèles qui en résultèrent. Probablement n'y a-t-il rien de plus difficile à réaliser qu'une liberté authentique.

8 Telle était l'une des raisons qui m'ont fait accepter la commande pour une œuvre vocale dont la première sera donnée en août de cette année au Festival international de musique chorale à Francfort. Cette manifestation sera consacrée à ce même sujet qui nous réunit aujourd'hui. J'ai décidé d'écrire la Fragen des Ode ${ }^{2}$ (Ode qui questionne) dont le texte serait composé seulement de quatre mots - traduits en plus de trente langues : LIBERTÉ ?/ÉGALITÉ ?/FRATERNITÉ ?/QUAND?

10 Il aurait été également possible de traduire les célèbres mots d'ordres de la Révolution Française dans beaucoup plus de langues et de dialectes, car les concepts dont il est question ici attendent partout encore leur réalisation adéquate et sincère. Ce choix ne représente pas une restriction mais est représentatif des conditions de vie dans tous les pays de ce monde. Cela implique que chacun des nombreux points d'interrogation a été précédé par une promesse brisée. Les lettres qui constituent cette dernière sont donc interchangeables.

11 La fin de l'œuvre s'avéra particulièrement difficile. Pendant la composition, passait dans ma tête, un peu comme une bande de film se répétant à l'infini, le bilan de mes expériences, de mes lectures, de mes observations. Tant d'images que je laissais ainsi passer en revue ne méritaient d'autre terme que celui, inventé avec une ironie salvatrice, par des Sud-Américains : démocrature.

Lequel de ces concepts, tous aussi prometteurs les uns que les autres, devait clore le texte ascétique du chœur? Le dernier terme serait peut-être interprété comme le bilan de ma prise de position, une indication de l'ordre d'importance que je trouvais dans la triade "Liberté - Egalité - Fraternité". A la fin de mon œuvre ne se trouvent ni la liberté, ni l'égalité, mais : "Fraternité. Quand ?"

\section{NOTES}

1. Kagel fait ici allusion au slogan nazi "Arbeit macht frei" [le travail rend libre] qu'il renverse dans la formule "Freiheit macht Arbeit". (NDR)

2. Création à l'Alte Oper de Francfort le 19 août 1989 pour la clôture du Festival choral international. 


\title{
Entretien avec Peter Eötvös
}

\author{
Philippe Albèra
}

Traduction : Daniel Haefliger

Comment voyez-vous la situation musicale actuelle, vous qui êtes en contact avec tant de musiques diverses?

Peter Eötvös : la vie musicale du $\mathrm{XX}^{\mathrm{e}}$ siècle semble être l'interminable prolongement de la vie musicale du XIX ${ }^{e}$. Nous assistons, pour des raisons bassement commerciales, à un dangereux mélange d'époque qui marginalise la vraie musique actuelle, une musique représentée par les œuvres et les compositeurs de notre temps qui continuent une évolution musicale qui doit bientôt aboutir au XXI ${ }^{\mathrm{e}}$ siècle ; il faut espérer que l'opinion retrouvera alors peu à peu l'identité de son époque.

Ce qu'on appelle l'"auditeur-consommateur" - cet étrange produit de notre siècle - est censé déterminer la situation musicale actuelle, dont on dit qu'elle est entrée dans une phase post-moderne : on ne peut plus faire de découvertes, mais seulement réaliser des mélanges plus ou moins réussis. Ce genre de considérations témoigne d'une étroitesse de vue considérable. D'une part, le facteur de "popularité" n'est pas déterminé par le public, mais il est imposé à celui-ci pour des raisons commerciales. En effet, la musique de répertoire rapporte davantage que la musique contemporaine ; elle nécessite peu de moyens techniques (l'effectif orchestral est stable), elle n'exige pas une préparation profonde, et remplit les salles grâce à une publicité gratuite due notamment au nombre considérable de représentations. D'autre part, un compositeur ne devrait pas se soucier des demandes de l'auditeur-consommateur, car c'est lui qui doit justement créer, puis assouvir cette demande. Il n'y a aucune fatalité à ce que la musique que l'on connaît soit ce qu'elle est: ce sont les compositeurs qui l'ont faite ainsi, à travers leurs recherches, leurs personnalités; ils n'ont pas répondu à une demande, et il serait illogique de voir les choses autrement.

4 Enfin, nous constatons que le facteur commercial est omniprésent dans toutes les formes et les structures musicales actuelles, et ce de manière si insistante, qu'il aveugle certains au point que ceux-ci justifient la prédominance de l'argent et de la publicité. Ce sont certes deux aspects essentiels de notre siècle, mais il faut de temps en temps rappeler qu'ils n'ont aucun rapport avec la musique. Ces conclusions erronées ont pour 
conséquence de faire stagner l'enseignement musical et de marginaliser le peu de musiciens capables de suivre les techniques contemporaines.

5 La musique contemporaine $a$ du succès partout où elle est bien organisée, où elle est instituée de manière conséquente et intelligente, où la forme de présentation est pensée; partout où la plus haute qualité d'exécution et une bonne acoustique coexistent. Je dois dire sincèrement que c'est rarement le cas. Très peu de salles peuvent répondre à ces exigences. Toutes les nouvelles salles de concert sont construites pour le répertoire du dix-neuvième siècle, les architectes ignorent en toute conscience les problèmes posés par la musique du vingtième. Il y a par exemple une "salle modulable" au Centre Pompidou que l'on ne peut modifier depuis dix années. Nous attendons tous la nouvelle salle de concert de la Villette qui, espérons-le, restera modulable!

6 Malgré les nouvelles compositions, les ensembles de musique contemporaine et les studios, il y a peu d'évolution du langage musical depuis les années cinquante-soixante. La génération de Boulez et de Stockhausen a créé le dernier langage fort. Elle était encore en accord avec son époque, alors que la musique actuelle comprend de nombreux mélanges - du "néo" au "post", de la simplicité à la complexité - sans faire jaillir un langage réellement adapté à notre temps. Et les œuvres récentes des compositeurs de la précédente génération ont acquis un caractère personnel, non représentatif du moment. Il faut espérer que l'exploitation efficace des nouvelles techniques, et notamment une maîtrise plus évoluée de moyens comme l'informatique, permettra une évolution importante au siècle prochain.

7 - Est-ce à dire que la recherche dans le domaine électro-acoustique - telle qu'elle se pratique à l'IRCAM par exemple - est à votre avis indispensable à l'évolution de la musique actuelle?

8 Peter Eötvös : La "recherche" n'est pas seulement la tâche de l'IRCAM ; c'est aussi une attitude fondamentale dans la vie. Rien n'est immobile; nous ne savons pas tout ; il y a toujours quelque chose à essayer, à ajuster, à interroger.

9 Je travaille en ce moment à l'IRCAM sur un projet ayant pour but de transformer les haut-parleurs en de véritables instruments, et d'améliorer leur qualité au point de pouvoir restituer un son authentique dans son timbre comme dans sa disposition spaciale. L'utilisation des haut-parleurs est actuellement seulement adéquat à la "popmusic", où ils ne servent qu'à amplifier et à rappeler, par une image déformée en quelque sorte, une sonorité originale - et non à restituer fidèlement le son. Le hautparleur étant, entre autres, le seul moyen de reproduction des sons synthétisés, ce projet me semble intéressant et être un bon exemple de recherche et d'évolution technique.

Les différentes familles instrumentales ont suivi un développement chronologique dans l'histoire. Des instruments actuels de l'orchestre, les cordes furent les premières à trouver leur forme définitive; elles n'ont pratiquement pas été modifiées depuis longtemps. Les bois et les vents se sont beaucoup développés depuis une centaine d'années, et évoluent encore aujourd'hui. Le dernier grand développement de ce siècle a concerné les instruments de percussion, et c'est maintenant le tour des instruments électroniques; j'espère ainsi développer les haut-parleurs jusqu'à obtenir d'eux une qualité de Stradivarius pour, entre autres, restituer les sons nouveaux. 
11 - L'introduction des instruments électroniques ne condamne-t-elle pas des structures institutionnelles telles que l'orchestre symphonique?

12 Peter Eötvös : L'assemblage instrumental de l'orchestre actuel n'est pas du dernier cri. Théoriquement, on pourrait varier ce son à l'infini; mais quelles que soient les variations, la teinte dix-neuvième siècle restera toujours présente: aucune des nouvelles "familles" n'a expulsé les anciennes; l'électronique est elle aussi intégrée "pacifiquement, lorsque sa qualité est d'un niveau suffisamment haut.

13 Nous devrions malgré tout ne pas perdre la liberté de réaliser, selon notre fantaisie, différentes combinaisons instrumentales - aussi librement que Berlioz ou Wagner, que Ives ou Varèse - et ne pas penser que la formation orchestrale actuelle est fixée pour l'éternité".

14 - Les oeuvres telles que Gruppen de Stockhausen, Dialoge de Zimmermann ou Figures, Doubles, Prismes de Boulez, qui tentaient à la fin des années cinquante de repenser la structure de l'orchestre, n'ont pas eu de suite. Pourquoi à votre avis?

15 Peter Eötvös : Pour des raisons administratives. Il y a beaucoup d'œuvres qui utilisent l'espace comme paramètre musical, mais elles ne sont jamais jouées parce qu'elles ne cadrent pas avec "la conception dix-neuvième". Elles exigeraient d'autres formes de salles de concert, et une autre forme d'organisation. On le sait ; on l'ignore. Gruppen est un chef-d'oeuvre et c'est pourquoi il est tout de même parfois joué malgré toutes les difficultés d'organisation. Ces exécutions sont souvent projetées avec enthousiasme, mais les deux tiers environ sont annulées peu avant leur réalisation ; par paresse.

16 - Comment vivez-vous la pratique de styles aussi différents et contradictoires que la musique de Reich, de Boulez, des néo-romantiques, etc.: trouvez-vous des points communs entre toutes ces musiques?

17 Peter Eötvös: Le point commun, c'est notre temps, c'est la liberté de penser différemment ; mais c'est aussi la rigueur des valeurs musicales, des structures claires et conséquentes, une grande énergie. Je ne vois là rien de "contradictoire". J'ai par contre personnellement une aversion pour toute réminiscence "néo".

18 - Quelle marge d'interprétation avez-vous dans une oeuvre contemporaine?

19 Peter Eötvös : Je pars du fait que l'oeuvre est plus importante que la personne de l'interprète. Une oeuvre est un tout fermé, c'est quelque chose qui est simplement "là", comme une étoile, comme un être vivant autonome. L'interprète doit mettre en jeu toute la maturité de sa personnalité, ses meilleures aptitudes au service de l'oeuvre ; et avec tant de liberté que l'oeuvre puisse tirer de lui tout ce qui jusqu'alors n'existait que de manière latente. Oeuvre et interprète doivent se fondre en une unité ; il ne faudrait jamais considérer l'interprète en dehors de l'oeuvre. Ainsi, il est préférable que les "stars" n'entrent pas du tout en contact avec les "oeuvres". Ce sont des personnes intéressantes, qui s'interprètent elles-mêmes; elles sont elles-mêmes des oeuvres d'art dont la forme d'apparition est la publicité.

20 Chaque compositeur sait exactement ce qu'il écrit, pourquoi et comment il l'écrit. Il n'y qu'une façon de lire une partition : celle qui est absolument exacte (ce qui n'est bien sûr pas aussi facile qu'on pourrait l'espérer).

21 - Le texte ne représente tout de même pas la totalité de l'oeuvre...

22 Peter Eötvös : La partition écrite n'est qu'une nécessité. La musique naît de la fantaisie, dans l'oreille intérieure du compositeur, et l'idéal serait de connecter une prise dans sa 
tête et de transmettre directement le son imaginé à un haut-parleur. Les compositeurs qui dominent en virtuose un instrument possèdent souvent cette possibilité directe de réalisation. Quand un son imaginé est réalisé par d'autres musiciens, il faut trouver une manière de communiquer. Jusqu'à présent c'était la notation musicale, mais elle ne doit pas rester l'unique possibilité. La notation musicale décrit la musique bien plus pauvrement que ce qu'elle était dans l'imagination; elle aide par contre à concevoir la structure, comme le plan de l'architecte. La comparaison plan/partition - maison terminée/musique exécutée, n'est somme toute pas si mauvaise.

Toute sa vie on essaie d'écrire de façon plus précise, plus diversifiée, plus univoque. Il reste malgré cela beaucoup d'éléments qu'on ne peut noter, qui ne peuvent être transmis que par la tradition - par la démonstration musicale, par des explications orales. C'est pourquoi je trouve très important que les compositeurs assistent à mes répétitions; je peux ensuite transmettre de première main leurs informations nonécrites.

Vous vous rendez compte ! il y a des chefs d'orchestre qui interdisent aux compositeurs d'être présents aux répétitions! Comment puis-je avoir confiance en eux quand ils dirigent la musique "traditionnelle" ?

Abstraction faite des virtuoses dont j'ai déjà parlé, il est devenu pour la première fois possible, grâce à la musique électronique, de réaliser la musique directement de sa propre main; comme un peintre, un sculpteur. Le compositeur peut bricoler son oeuvre le temps qu'il veut, jusqu'à ce qu'elle sonne exactement telle qu'il se l'était imaginée. C'est enregistré, mémorisé, ça ne bouge pas, comme au cinéma. Mais bien qu'il sera possible, grâce à l'ordinateur, de simuler beucoup d'instruments dans un futur proche, cela n'exclut naturellement pas les représentations "live" avec des musiciens, ce qui est une chose tout autre et tout aussi importante. C'est un peu comme au théâtre : la représentation devient aussi importante que la pièce.

- Vous semble-t-il qu'aujourd'hui les compositeurs attachent plus d'attention qu'autrefois aux questions de la perception?

27 Peter Eötvös : La perception est une question d'observation aiguë, de clarté de la conscience, de précision des organes - ici de la précision des oreilles. Les anciens instruments à percussion de l'est, par exemple, témoignent d'un sens de 1'écoute très raffiné et d'une maîtrise artisanale invraisemblable; durant toute l'histoire de la musique, le constant changement et développement des instruments de musique montre que les hommes ont toujours écouté très attentivement. Des compositeurs comme Machaut, Berlioz, Debussy étaient respectivement au niveau de perception le plus haut de leur époque. Et aujourd'hui comme par le passé : Boulez ou Stockhausen ont les oreilles aussi sensibles que celles des chauve-souris. Il y a des gens qui sont tellement sensible à l'articulation que cela en devient pénible: Leopold Mozart à l'époque (on le sait d'après ses écrits), ou Kurtag aujourdhui.

28 Le but du compositeur est justement de vouloir communiquer aux "autres oreilles" ses découvertes, sa sensibilité spécifique.

29 - En tant que compositeur, comment conciliez-vous la pratique de tant de musiques différentes avec votre propre création?

30 Peter Eötvös : Je n'essaie absolument pas de trouver un équilibre. Comme compositeur, j'ai un plan précis fixé à l'avance qui porte sur des années, et j'essaie actuellement de réduire mes activités de chef d'orchestre de manière à n'être pas qu'un compositeur de 
week-end, mais à pouvoir trouver toujours plus de temps pour penser et écrire. Dans ma production, chaque pièce a sa propre langue, possède son propre monde sonore.

En dirigeant, j'ai beaucoup appris sur l'instrumentation, le maniement des instruments, l'articulation et l'organisation du travail. Ce sont des expériences positives que je peux mettre en valeur dans mes compositions.

- Parmi les jeunes compositeurs actuels, quelles sont les personnalités qui vous paraissent les plus originales?

33 Peter Eötvös : En tant que chef d'orchestre, cela me rend très heureux d'avoir des contacts aussi directs que nombreux avec les compositeurs. Je voyage beaucoup et connais relativement bien ce qui s'écrit dans les différents pays. Je suis très curieux de savoir comment pense ma génération, ce que les jeunes envisagent pour le futur. Avant, j'étais très idéaliste et j'aimerais bien le rester. Ce sont les oeuvres fortes des jeunes compositeurs qui comptent pour moi : on trouve souvent dans la production de ceux-ci des oeuvres originales, mûres ; mais par la suite, ce ne sont plus que des "préparations" esquissées ou de simples répétitions.

34 Chaque année, je lis environ mille partitions. Dans une composition, je recherche par exemple : une conception sonore précise, une capacité formelle, une connaissance des instruments, une force "dramatique" du discours, une conception de l'articulation. Ces deux dernières qualités manquent la plupart du temps. La musique est une forme de communication, un dialogue actif, pas un “divertissement". Trop de gens ne veulent pas le comprendre, parce qu'ils ne touchent la musique qu'en surface. La bonne musique était de tout temps une recherche; et c'est encore valable aujourd'hui !

- Pensez-vous que les musiques extra-européennes ou le jazz par exemple peuvent constituer un apport important dans la composition?

Peter Eötvös: J'espère! Pour ma part, j'aimerais penser un monde unifié, avec différentes individualités, et avec une connaissance réciproque. L'influence ou la connaissance d'autres musiques, c'est avant tout la reconnaissance que l'autre existe. Les mélanges m'intéressent si l'on ne s'en tient pas seulement à des éléments rapportés. Moi-même, je suis très influencé par le jazz, que j'écoute beaucoup, que j'adore, mais je n'utilise pas ce langage-là. Je crois qu'on peut se comprendre entre personnes différentes sans vouloir être l'autre.

- Qu'est-ce qui vous intéresse dans le jazz?

39 Peter Eötvös : C'est le jeu des éléments prévisibles et les surprises. Un aspect qui vaut aussi pour la musique de Mozart : dans son langage, il y a une logique qui me procure des attentes et qui crée des surprises : ainsi, nous sommes toujours en discussion avec l'oeuvre.

Dans notre culture musicale européenne, nous essayons de créer dans chaque oeuvre un monde nouveau, de représenter le jamais-entendu, de façon à ce que l'auditeur soit constamment attentif au point de pouvoir suivre une histoire inconnue dans une langue inconnue. (C'est comme ça que je me sens au Japon, dans un théâtre Nô.)

41 Lorsque ces oeuvres emploient un "code-système" très clair, une première audition, peut-être, peut suffire à rendre clairement le discours. Bien sûr, il y a beaucoup d'archétypes dans chaque pièce, d'éléments qui facilitent l'orientation : par exemple, le système de son ou la caractéristique de l'instrumentation, la gestique. 

langue connue; à l'audition, je ne suis plus un observateur, j'établis un dialogue réciproque avec la musique, je suis totalement "dedans", je dois seulement en connaître la langue.

43 lorsqu'on s'y applique avec un esprit de recherche, et que la préparation peut se faire dans les meilleurs conditions, comme c'est le cas pour Kleiber, Celibidache ou Harnoncourt.

En leur temps, Busch et Reiner préparaient soigneusement les oeuvres du répertoire ; il $\mathrm{y}$ avait beaucoup de répétitions, et l'esprit de recherche avec lequel il travaillaient débouchait littéralement sur la recréation de l'oeuvre. C'est comme lorsqu'une pièce de théâtre du répertoire est mise en scène par un metteur en scène contemporain : c'est un événement.

De nos jours, la plupart des concerts de musique classique sont bâclés - je ne vise pas ici la qualité des chefs d'orchestre, mais leur attitude professionnelle; si l'on joue trop souvent la même chose sans y faire attention, la musique se transforme en prière rabâchée, en rituel sans fond et sans intérêt.

Imaginez que l'on ne joue plus la Neuvième Symphonie pendant trois ans par exemple, et qu'après, les meilleurs chefs d'orchestre, avec les meilleurs orchestres et dans les meilleures conditions, fassent une tournée mondiale avec elle. En tant qu'événement musical et culturel, cette façon d'agir serait beaucoup plus saine et raisonnable, et dans ce cas l'intérêt serait bien plus musical que commercial.

47 En fait, j'ai dirigé beaucoup d'oeuvres de la fin du XIX ${ }^{e}$ siècle dans le cadre de ma collaboration avec l'orchestre de la BBC, et prochainement je dirigerai Wagner, Brahms et Mozart; mais je veillerai chaque fois à ce que les conditions et l'état d'esprit nécessaires à la musique soient respectés; et je ne chercherai pas des prétextes pour remplir les salles. 


\title{
Entretien avec Diego Masson
}

\author{
Philippe Albèra
}

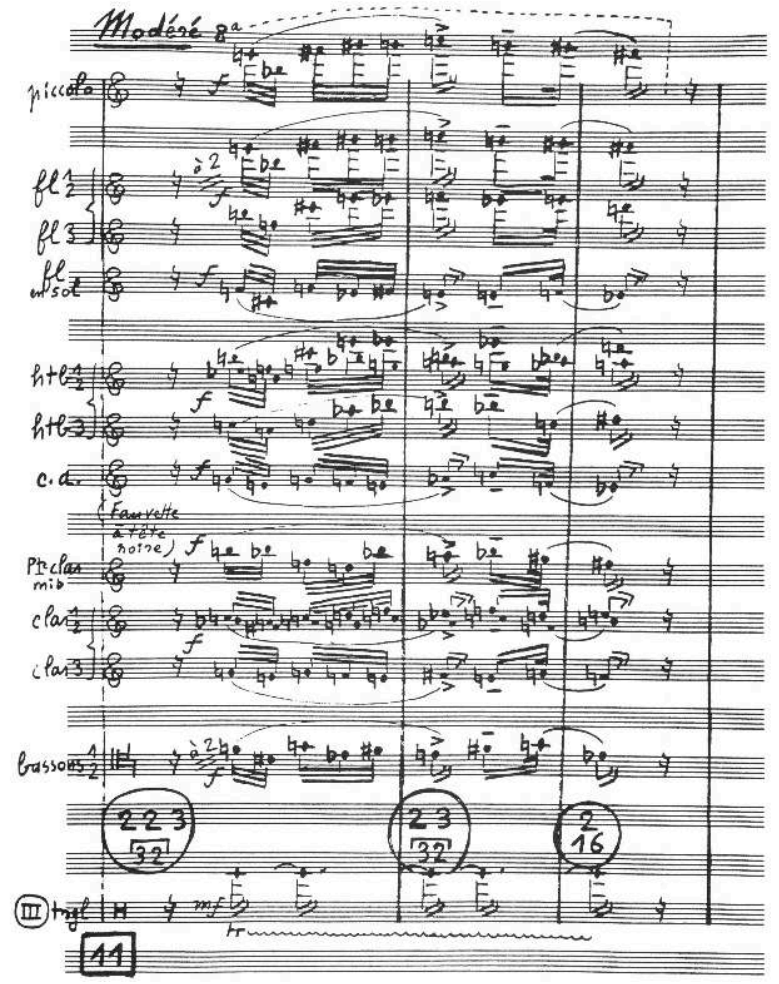




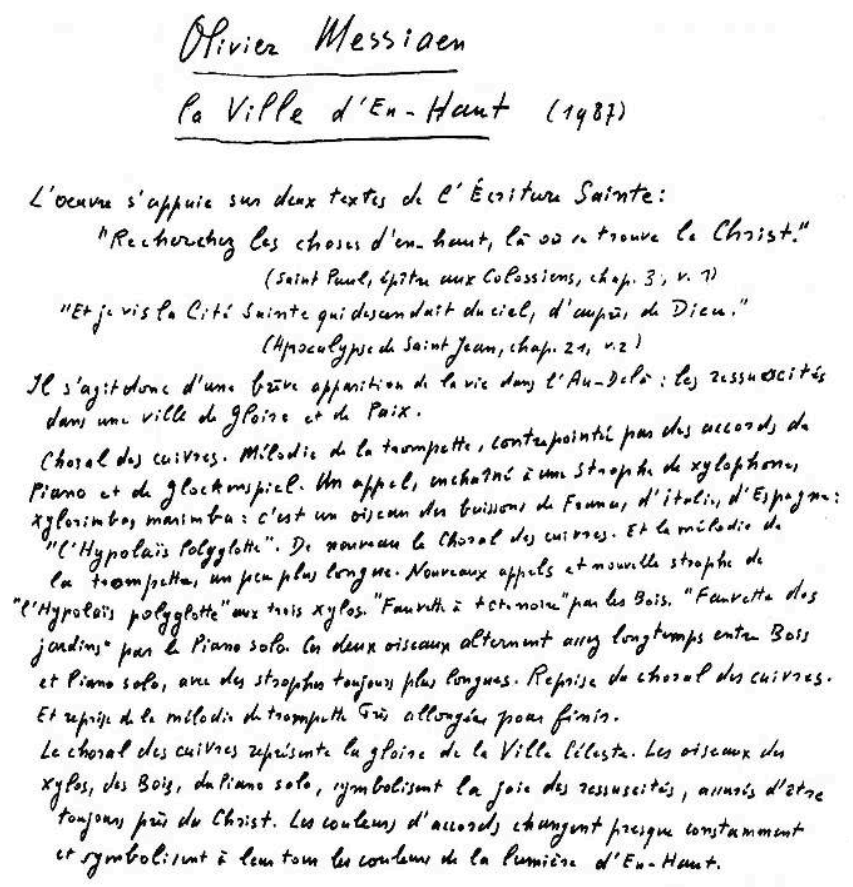

\section{OPivier Messiaen}

1 Vous avez fait beaucoup de créations dans votre carrière de chef, et vous avez incarné un esprit avec "Musique Vivante" dans les années soixante et soixante-dix; aujourd'hui, on vous voit plus rarement à Paris. Pourquoi?

2 Diego Masson: Je fais toujours autant de créations qu'auparavant, mais davantage en Angleterre qu'en France. Cela tient simplement au fait que nous avons de gros problèmes budgétaires à "Musique Vivante", et que c'est avec cet ensemble, dans ce cadre-là, que je veux travailler en France. Comme on ne nous donne plus beaucoup de moyens, je ne fais pas grand-chose...

3 - Trouvez-vous que la situation a beaucoup changé par rapport à il y a vingt ans ?

4 Diego Masson : Il y a vingt ans, on avait des idées claires, on avait l'impression de savoir où l'on allait, il existait un "style" regroupant les compositeurs qui nous intéressaient à l'époque, avec des individualités, bien sûr. Aujourd'hui on sait à quoi s'opposer, mais on ne sait plus où aller : il n'y a plus de tendances, de styles, de formes ou de langages en commun; il n'y a plus que des compositeurs individuels qui ont chacun leur propre tendance, qui varie d'ailleurs parfois d'une œuvre à l'autre. Je crois que cela est lié à un phénomène socio-politique plus général : pour ma part, je ne sais plus vers quoi me diriger! J'ai les mêmes idées qu'auparavant, mais j'ai l'impression aujourd'hui d'être tout seul! Pour les compositeurs, c'est la même chose : chacun a ses idées, mais il n'y a plus d'idées communes. Il n'y a même pas de débat. D'ailleurs, c'est comme en politique où il n'existe plus que des faux débats, des pseudo-débats.

5 - Comment ressentez-vous l'évolution des compositeurs que vous défendiez dans les années soixante?

Diego Masson: C'est extrêmement variable! Il y en a qui continuent leur chemin, comme Boulez et Xenakis par exemple: ce qu'ils écrivent aujourd'hui est différent de leurs œuvres anciennes, mais il y a une logique d'évolution. D'autres ont pris un chemin qui représente quasiment ce contre quoi on se battait alors, comme 
Stockhausen : il fait de la musique aujourd'hui qu'on aurait combattue dans les années soixante.

7 - Quand avez-vous perçu un tel changement dans la musique contemporaine, comment l'expliquez-vous?

8 Diego Masson : Je pense que cela date d'après 68. Le changement m'a semblé toutefois insidieux, sans déclarations de principes. Je pense que cela est lié à un éclatement, non seulement de la musique, mais de la société tout entière. Mais cette absence d'école n'empêche pas qu'il y ait des choses formidables aujourd'hui! Cela dit, je ne crois pas que, pour les années cinquante, on puisse parler d'un groupe organisé ; je crois plutôt que nombre de compositeurs partageaient certaines idées communes.

9 - Qu'avez-vous envie de défendre aujourd'hui ?

10 Diego Masson: C'est purement subjectif, puisqu'il n'y a plus de critères! Je suis personnellement attiré par des œuvres qui reposent sur une idée formelle forte, et qui sont sous-tendues par une réelle cohérence stylistique. A part cela, je crois que je m'en remets au plaisir auditif !

11 - Vous vous basez sur des critères liés à la tradition en quelque sorte ; mais ne croyezvous pas que l'on puisse se chercher à l'écart de cette tradition, dans le mélange des cultures ou des types de musique, par exemple?

12 Diego Masson: A ce moment-là, c'est un abandon des critères avec lesquels nous vivions, mais je ne vois pas à quels critères nouveaux de tels compositeurs se rattachent. Je ne vois aucune influence réelle de musiques non européennes sur la musique européenne actuelle, sinon des influences que j'appellerais touristiques.

13 - Pour vous, quel est le rapport de la musique actuelle avec la tradition, notamment en comparaison avec l'attitude qui prévalait dans les années cinquante et soixante?

14 Diego Masson: J'ai l'impression, en réalité, que dans les années cinquante et début soixante, on se rattachait à une certaine tradition musicale classique - Bach, Mozart, Beethoven - alors qu'actuellement, on se rattache plutôt au XIX ${ }^{\mathrm{e}}$. C'est le sentiment instantané qui importe. Par exemple, on trouve aujourd'hui des musiques qui se rapportent à quelque chose comme les concertos de Tchaïkovski, qui pour moi sont incompréhensibles musicalement, qui me semblent essentiellement des sortes d'improvisations. Dans les années cinquante, on était davantage attiré par les formes strictes d'une tradition plus ancienne. Mais c'est peut-être aussi le poids des commandes, plus nombreuses aujourd'hui pour l'orchestre ou pour les solistes qu'autrefois.

15 - Votre expérience de chef vous confronte-t-elle toujours à des résistances de la part des instrumentistes, ou bien la situation s'est-elle transformée aujourd'hui?

Diego Masson: Dans les orchestres symphoniques il y a toujours une résistance des musiciens, les choses n'ont pas beaucoup changé. Quant aux groupes spécialisés, la situation a nettement évolué : les musiciens jouent aujourd'hui sans trop de difficultés des œuvres qui dans les années soixante exigeaient énormément de répétitions. Et cela même avec des ensembles jeunes, qui n'ont pas une grande connaissance du répertoire contemporain! Il y a presque une assimilation par osmose, que l'on trouve chez des musiciens ne pratiquant pas forcément ce répertoire. Lorsque j'ai commencé à jouer de la musique contemporaine, vers 1954, en tant que percussionniste, je trouvais bien difficile la plupart des partitions; aujourd'hui cela parait presque facile. Il est donc 
possible aujourd'hui de monter les œuvres contemporaines plus vite et mieux qu'auparavant.

Il me semble qu'on observe le même phénomène au niveau du public : les gens, même sans être des "habitués" de cette musique, l'écoutent comme n'importe quelle autre musique; il n'y a plus cette impression d'agressivité, cette tension qui existait autrefois. C'est notamment le cas lorsque vous jouez une œuvre contemporaine dans un programme classique d'orchestre par exemple : on ne retrouve pas les réactions d'il y a vingt ou trente ans. Le problème, c'est plutôt de faire venir ce public!

18 - Comment expliquez-vous que la peinture moderne, par exemple, ait su conquérir un assez vaste public, alors que la musique contemporaine reste confinée à des petits groupes d'initiés?

Diego Masson : L'engouement du public pour la peinture moderne correspond-il à une véritable passion, à une véritable connaissance ? L'avantage de la peinture, c'est qu'on peut rester dix secondes devant une toile - c'est à peu près la moyenne dans les grandes expositions - et que, si vous n'aimez pas un tableau, vous pouvez passer au suivant. Dans la musique, vous êtes obligés de rester jusqu'à la fin de l'œuvre, vous êtes obligés d'écouter : c'est plus astreignant. Il y a dans les expositions un phénomène de mode évident, et aussi un aspect financier : les tableaux représentent de plus en plus des valeurs considérables, qu'on a envie de voir en tant que telles. Il vaudrait mieux comparer la musique à la littérature contemporaine : peut-on parler d'un véritable engouement du public à son égard? Je ne crois pas !

20 Je crois tout de même qu'il y a plus de public pour la musique contemporaine qu'autrefois. Dans les années cinquante ou soixante, les concerts étaient peut-être pleins : mais il y en avait très peu. Aujourd'hui, l'offre est considérable! Mais il y aurait encore davantage de public si les moyens financiers de la musique contemporaine atteignaient ceux de la musique classique. On dépense des millions de publicité et de promotion pour "vendre" un opéra de Gounod qui est archi-connu; et pour un jeune compositeur pas encore reconnu, vous avez trois sous pour faire la promotion.

21 - Vous trouvez que l'organisation de la vie musicale est très stagnante?

22 Diego Masson : Oui, sans aucun doute. Les pouvoirs publics devraient jouer leur rôle, et ils ne le jouent guère. Alors, on vous dit que la musique contemporaine intéresse une minorité de personnes. Mais n'importe quelle activité aujourd'hui n'intéresse qu'une minorité de personnes ! La musique classique par exemple. Le football aussi ! Le RacingClub de Paris coûte des millions chaque année, et il n'attire qu'une moyenne de dix mille ou douze mille spectateurs par mois : c'est peu de chose, par rapport au coût! En réalité, le rapport entre le coût d'un concert de musique contemporaine et le nombre de spectateurs qui s'y rend est bien supérieur à celui d'un match de football du Matra Racing, ou d'une représentation d'un opéra de Gounod!

- Vous me disiez que "Musique Vivante" n'a plus guère de moyens pour réaliser une véritable saison. Si tel était le cas, qu'en feriez-vous?

Diego Masson : Il est difficile aujourd'hui de réaliser un concert qui ait une unité de style.

25 - La spécificité des musiciens de "Musique Vivante", qui pratiquent différents genresthéâtre musical, improvisations, jazz, etc. - reste importante aujourd'hui? des genres avait son utilité à une époque où existait une unité très forte, mais 
aujourd'hui qu'elle n'existe plus, c'est devenu secondaire. C'est vrai qu'aux débuts de "Musique Vivante", j'essayais de trouver des musiciens qui avaient une autre formation que celle de la seule musique classique, mais aujourd'hui ce n'est plus important. Pour nous, ce sont des caractéristiques qui viennent du passé. En revanche, ce qui me semble toujours essentiel à "Musique Vivante", c'est la notion de plaisir. J'ai toujours pensé que la musique contemporaine, c'est aussi un énorme plaisir : à jouer et à écouter. Je crois que le plaisir très intense que nous avons à faire de la musique ensemble est ressenti par le public qui nous entend. Pour moi, l'un des plaisirs de la musique contemporaine, c'est aussi celui de la découverte: diriger une œuvre nouvelle, ou l'œuvre d'un compositeur que je ne connais pas, m'excite terriblement.

- Quelle est la part d'interprétation dans une œuvre nouvelle?

Diego Masson : La même que dans une œuvre classique. Ce n'était pas le cas dans les années cinquante ou soixante, parce qu'on avait du mal à jouer les notes. Mais, aujourd'hui, ce n'est plus un problème, et on se concentre sur l'interprétation. Et je crois que pour les auditeurs, l'écoute est plus agréable, plus musicale. On rend bien davantage justice aux œuvres : lorsqu'on joue maintenant Kontrapunkte de Stockhausen, par exemple, on voit combien c'est de la belle musique, alors qu'autrefois, on trouvait peut-être que l'œuvre ne "sonnait" pas très bien parce qu'on n'arrivait pas à la dominer instrumentalement. On voit aussi comment les compositeurs eux-mêmes modifient leur conception : regardez Boulez avec le Marteau sans maitre : entre la première partition et la partition révisée, entre les premières interprétations et celles d'aujourd'hui, il y a une énorme distance. Finalement, le musicien a la même latitude avec une indication telle que "la noire à 132" et l'ancien "Allegro vivace".

Ce qui, par contre, est dramatique, c'est que les orchestres symphoniques n'aient pas à leur répertoire toute la musique de la première moitié du $\mathrm{XX}^{\mathrm{e}}$ siècle, et une grande partie de celle des trente dernières années; il y a vraiment là un blocage incompréhensible et inquiétant. Lorsque vous proposez à un organisateur ne serait-ce qu'une œuvre de Schoenberg, il vous dit: "Surtout pas, car cela va vider la salle!" En fait, il n'en sait rien puisqu'il ne programme jamais Schoenberg. Je pense que c'est une des raisons pour lesquelles le grand public n'écoute pas la musique contemporaine : elle est maintenue dans un ghetto. Or je crois que les organisateurs se trompent: j'ai vu dernièrement, par exemple, Abbado faire les Gurrelieder de Schoenberg avec l'Orchestre des Jeunes de la Communauté européenne, et la salle était archi-comble, les gens se battaient pour entrer!

30 - Cette musique ne serait-elle pas différente si elle était intégrée vraiment dans l'institution, alors qu'elle s'est souvent opposée à celle-ci, surtout dans les années soixante?

31 Diego Masson : Je n'ai jamais perçu, pour ma part, la musique des années cinquantesoixante comme une musique qui s'opposait à la tradition classique. Elle ne s'opposait ni à Mozart, ni à Beethoven, ni à Wagner, mais à une fausse tradition académique du $\mathrm{XX}^{\mathrm{e}}$ siècle. Jouer Beethoven et Berio au même programme ne pose aucun problème, alors que mettre par exemple Landowski et Berio, oui, cela pose un problème ! - Les différents ensembles de musique contemporaine ne devraient-ils pas alors intégrer aussi la musique classique dans leur répertoire? Diego Masson : Non, je ne crois pas que ce soit leur rôle. Pour deux raisons : l'une, c'est que les formations instrumentales ne coïncident pas; les formations propres au 
répertoire contemporain ne permettent pas de jouer la musique classique. L'autre, c'est que le public de la musique classique ne vient pas de toute façon; j'ai fait cette expérience une fois lors de concerts au Palace : il a été négatif. d'écrire aujourd'hui des formes sonates ou des fugues, mais ce qui est important, c'est l'idée qu'une forme logique et cohérente est indispensable pour organiser une œuvre. Cette idée fait partie de notre culture, et pas seulement en musique. Ce qui veut dire, pour moi, que lorsque les compositeurs européens suivent quelqu'un comme John Cage, ils font fausse route. Entendons-nous bien : je trouve souhaitable qu'il existe plusieurs sortes de musiques, je ne suis pas pour l'uniformité! œuvres avec électronique. Je ne crois pas vraiment que l'électronique musicale soit un domaine fondamental. Peut-être suis-je un peu vieux jeu sur ce point! Regardez Répons par exemple: je trouve que c'est une œuvre magnifique, mais pas à cause de l'électronique ; par l'écriture elle-même. 


\title{
Entretien avec le Quatuor Arditti
}

\author{
Philippe Albèra
}

1 Comment est née la décision de vous consacrer exclusivement au répertoire contemporain?

2 Irvine Arditti : Je m'intéresse depuis toujours à la musique contemporaine. Lorsque j'étais étudiant à Londres, nous pouvions écouter de nombreux concerts, notamment à la BBC que dirigeait alors Boulez. En 1968 - j'avais quinze ans - je suis allé à Darmstadt. Bien sûr, la plupart de mes collègues, dans les instruments à cordes notamment, étaient extrêmement critiques vis-à-vis de la musique contemporaine. C'est pourquoi, en créant le quatuor Arditti, un peu par hasard d'ailleurs, je voulais non seulement jouer la musique nouvelle, mais le faire avec plaisir. Notre première expérience fut liée à un concert Penderecki à la Royal Academy of Music, en présence du compositeur. C'est ainsi que nous avons commencé. Petit à petit, nous nous sommes créé une réputation en jouant le répertoire contemporain et en travaillant avec les compositeurs, directement.

3 - Ne pas jouer les œuvres du répertoire classique et romantique pour quatuor à cordes constituait un choix dès le départ?

4 Irvine Arditti : Oui. Ce fut un choix positif. Il existait - et il existe toujours - beaucoup de quatuors jouant le répertoire, et de nombreux quatuors anglais jouaient les œuvres de Britten, Tippett, Chostakovitch...... Nous voulions nous consacrer aux œuvres que l'on ne jouait pas. Certes, nous avons aussi travaillé les "classiques" du vingtième siècle : il est important de ne pas se limiter à la seule musique nouvelle! Mais nous manquons de temps pour aborder sérieusement le répertoire traditionnel, même si nous aimerions le faire! Regardez les autres quatuors : ils ne jouent souvent qu'une ou deux œuvres nouvelles par an.

5 - Quels problèmes avez-vous rencontrés en jouant ce répertoire nouveau avec la formation classique que vous aviez reçue du Conservatoire?

6 Irvine Arditti : Les premiers membres du quatuor, qui étaient tous mes amis, ont pu avoir des problèmes en ce sens. Pour moi, le langage contemporain était familier, naturel. J'aimais écouter la musique de Boulez, Xenakis, Ligeti, etc., et j'étais fasciné par 
le fait de pouvoir la jouer. J'ai ressenti comme une mission de les encourager à écrire pour quatuor à cordes.

7 Rohan de Saram : Dans mes premières années de carrière, je n'ai joué que le répertoire traditionnel. Mon premier contact avec la musique contemporaine s'est fait à l'occasion d'une invitation à jouer une œuvre de Xenakis pour la Radio des Pays-Bas. J'ai dû reprendre mon étude du violoncelle à partir de zéro pour aborder cette pièce, qui reste l'une des plus exigeantes du répertoire. Pour moi, les premières années avec le quatuor Arditti ont été difficiles, parce que je n'avais aucune expérience de la musique contemporaine. J'ai dû apprendre de nouvelles techniques, de nouvelles façons de jouer.

8 Levine Andrade : Personnellement, je trouve le répertoire contemporain beaucoup plus intéressant que le répertoire classique, que j'ai pratiqué plusieurs années : c'est en effet un défi pour l'esprit, il y a beaucoup plus de difficultés à surmonter !

9 David Alberman: Si l'on joue dans un orchestre, on représente toujours une petite partie d'un tout. Le processus selon lequel l'interprétation se construit n'est absolument pas démocratique : c'est le chef qui en décide. Et d'un certain point de vue, cela n'est pas très intéressant, il n'y a pas de défi à relever pour l'instrumentiste. Avec le quatuor Arditti, je trouve fascinant d'être confronté à une musique qui n'a jamais été jouée, et de devoir construire complètement l'interprétation, voire d'inventer de nouvelles techniques de jeu réclamées par l'œuvre. En effet, si les compositeurs classiques étaient à l'aise avec leur notation, les compositeurs d'aujourd'hui la ressentent souvent comme un obstacle, et c'est à nous de trouver ce que le compositeur veut, et la manière de l'obtenir. Cela m'apporte une réelle satisfaction musicale, et m'oblige à penser, bien davantage en tous les cas que lorsque j'étais membre d'un orchestre!

10 Irvine Arditti : C'est vrai : lorsque l'on aborde le répertoire classique, on est d'emblée confronté à une tradition d'interprétation, qu'on le veuille ou non. On sait où se trouvent les limites. Notre travail est différent : nous ne pouvons pas nous appuyer sur une tradition quelconque. Nous devons la créer. Nous devons aussi aider les compositeurs à réaliser leurs idées: certains ont besoin de telles orientations techniques ; parfois, des œuvres qui "fonctionnent" sur le papier posent des problèmes à l'écoute. En réalité, nous sommes complètement impliqués dans le processus de création.

11 - Quelle est votre première approche d'une cuvre nouvelle, comment vous y prenezvous?

12 Irvine Arditti: Au début, nous avons une approche très mécanique. Comme nous voulons éviter toute interruption dans le jeu, notamment à cause des tournes de pages, nous recréons la plupart du temps les partitions. Nous inscrivons les signaux nécessaires pour le jeu d'ensemble. Et nous étudions la partition, autant que possible, avant de jouer, afin de comprendre ce que le compositeur a voulu. Par exemple, il existe différentes façons de noter les sons non traditionnels, ou les différentes sortes de "bruits" : nous devons comprendre ce que le compositeur désire exactement, et trouver les moyens techniques de l'obtenir. Nous proposons aussi parfois des solutions qui nous paraissent meilleures... Mais il existe des compositeurs plus ou moins "dogmatiques", plus ou moins têtus : il est parfois nécessaire de jouer quelque chose qui ne sonne pas très bien, parce que cela est organique avec l'idée de l'œuvre. Tous ces problèmes se 
règlent de cas en cas, par un travail complexe, par des échanges nombreux, par l'expérience.

13 Rohan de Saram : Je crois que ces problèmes entre la partition et le résultat audible, ou entre le compositeur et l'interprète, proviennent de ce que les compositeurs ne sont plus, comme autrefois, eux-mêmes instrumentistes. A l'époque baroque, et même encore à l'époque classique, les compositeurs jouaient de plusieurs instruments, ils avaient des connaissances pratiques. A l'époque de Wagner, ils sont devenus chefs d'orchestre. Aujourd'hui, ils ont souvent peu de liens avec la pratique musicale. C'est le développement même de la musique occidentale : cela a permis d'élargir son horizon à d'autres domaines, comme la philosophie ou les mathématiques, mais en même temps cela pose des problèmes sur le plan de la pratique instrumentale. Les idées musicales ont beaucoup évolué, alors que nous jouons toujours sur les instruments de l'époque de Jean-Sébastien Bach !

14 Irvine Arditti : Oui, mais la conception de leur utilisation a changé, parfois de manière radicale.

Rohan de Saram: Pour nous, il est nécessaire de savoir distinguer entre les compositeurs qui ont des connaissances très détaillées de la technique instrumentale, et ceux qui ont des connaissances plus superficielles. Nous pouvons aider les seconds à mieux réaliser, à mieux concrétiser leurs idées. Il faut accepter le fait que nous sommes dans une époque sans traditions héritées de ce point de vue, dans une époque de décomposition des valeurs traditionnelles.

16 - Faites-vous un travail d'analyse sur les partitions, pour en dessiner les hiérarchies, l'architecture, la signification?

17 Irvine Arditti : La musique contemporaine pose souvent des problèmes différents de ceux de la musique classique : on ne peut pas lui appliquer le même type d'approche enfin, pas toujours! Nous n'avons pas besoin de disséquer une œuvre pour savoir comment elle est écrite, mais il est évidemment essentiel que nous sachions le sens de chaque élément que nous jouons.

18 David Alberman: Pour construire une interprétation, nous avons besoin de savoir quelle est la forme dramatique de l'œuvre, ce qui implique d'être au clair sur sa construction. Il nous faut identifier les différents éléments, les différentes structures de l'œuvre, afin de les placer à l'intérieur du schéma dramatique. Nous analysons non pas comme des universitaires, comme on découperait un cadavre, mais dans l'idée que l'œuvre est un organisme vivant, et qu'il faut lui donner une cohérence, une forme perceptibles. Nos décisions en ce sens s'appuient d'abord sur ce que le compositeur a écrit. Et puis nous sommes à l'écoute les uns des autres: l'élaboration de l'interprétation se fait d'une manière très démocratique. Nous suivons celui qui réagit le plus passionnément à tel ou tel passage! Nous sommes également attentifs à ce qui fonctionne ou non durant le concert, et en ce sens, nous nous déterminons sur la base de notre expérience.

Irvine Arditti: Nous avons des rapports d'extrême franchise entre nous, ce que je trouve très important: qu'il s'agisse de la manière de jouer, d'un problème d'intonation, ou de quoi que ce soit d'autre. Il est d'ailleurs plus facile de juger ce que l'on entend que ce que l'on joue.

20 Rohan de Saram : Je trouve extrêmement positif que nous jouions d'abord pour nous satisfaire nous-mêmes. En tant que soliste, on projette beaucoup le son vers l'extérieur, 
et cela ne permet pas de se mettre soi-même en question. Je me souviens que, enfant, j'avais joué pour Casais ; j'avais environ douze ans, et j'ai interprété une suite de Bach. Casals m'a dit, à propos d'un passage : "Jouez cette phrase pour moi” - ce que j'ai fait. Puis il m'a dit : "Jouez cette phrase pour vous-même” - et je l'ai jouée encore une fois, sans bien m'apercevoir de la différence. Casais m'a alors dit: "Vous avez mieux joué pour vous-même que pour moi”. Autrement dit, j'avais joué avec une intériorité et une conviction plus grandes.

21 - Certaines démarches compositionnelles, je pense par exemple à celle de Lachenmann, n'entrent-elles pas en contradiction complète avec la structure de ces instruments qui n'ont pas changé depuis 300 ans?

Irvine Arditti : Je plains beaucoup quelqu'un comme Lachenmann : il sait exactement ce qu'il veut, il suit une direction très précise, mais il travaille dans un monde sonore qui s'éloigne complètement de la norme existante. On pourrait dire que ce qu'il demande va contre les instruments, mais cela ne me dérange pas dans la mesure où le résultat est musical. Certaines techniques qu'il exige représentent l'antithèse de la façon traditionnelle de jouer de nos instruments. Souvent, en nous donnant des exemples de ce qu'il veut, il obtient des résulats meilleurs que nous, alors qu'il n'est pas instrumentiste à cordes! En fait, il ne subit pas l'influence d'une tradition de jeu. Cela nous oblige à apprendre certaines choses, comme des débutants; on peut dire : ce sont des bruits qu'un enfant pourrait faire lui-même, mais ce n'est pas vrai, ils sont très difficiles à réaliser, à maîtriser !

23 - De telles exigences vous apparaissent-elles comme une continuité de la tradition, ou comme une rupture avec elle?

Irvine Arditti : Il est difficile de les considérer comme continuité de la tradition...

David Alberman: Lorsque Haydn a commencé d'écrire ponticello dans l'une de ses Symphonies, il a fait scandale. Bien plus tard, lorsque Schoenberg a demandé que le ponticello se fasse complètement sur le chevalet - autrement dit, il voulait un bruit - cela a créé des problèmes. Lachenmann n'est pas le premier à travailler sur différentes sortes de bruits. Mais il est vrai qu'il y a une sorte de dislocation du monde sonore traditionnel; nous entrons avec lui dans un nouvel univers sonore, qui est à mon avis d'une grande pureté. Il n'a plus les mêmes fondements que l'univers sonore traditionnel. Lachenmann a porté une très grande attention aux différents "bruits", et si 1'on devait construire une machine capable de les produire - en admettant que ce serait plus rationnel que d'utiliser des violons - cette machine ressemblerait finalement à un violon!

26 Irvine Arditti : Je crois qu'il faut se méfier du mot "bruit", qui est souvent associé à quelque chose de désagréable. Ce qui est important, c'est que le résultat du travail de Lachenmann est une œuvre de musique. Pour moi, les démarches très différentes de Ferneyhough, Xenakis ou Lachenmann se rattachent toutes à la même tradition de faire de la musique.

27 - Ne risquez-vous pas d'imposer un même style à l'ensemble des œuvres que vous jouez?

28 Irvine Arditti : J'aimerais croire que le quatuor n'a pas un son fixe, car il me semble impossible de jouer de la même façon des œuvres qui appartiennent à des mondes sonores complètement différents. Lorsque nous jouons Ferneyhough ou Carter, Xenakis 
ou Nono, nous essayons à chaque fois de projeter un certain type de sonorité. Evidemment, on ne peut pas changer complètement sa manière de jouer !

David Alberman: Ce qui peut aussi varier, c'est la manière de phraser, la gamme dynamique, etc. Ce qui est merveilleux dans notre répertoire, c'est la variété qui existe dans ces domaines.

- Comment s'effectue le choix de votre répertoire, quels sont vos critères pour accepter ou refuser de jouer une œuvre nouvelle?

Irvine Arditti : Souvent, le répertoire est choisi par les organisateurs, les producteurs : ce sont eux qui font les commandes ou qui décident d'un programme. Nous essayons de garder une position aussi ouverte que possible. En général, il est assez facile de se mettre d'accord sur une pièce qui est mauvaise; mais nous ne partageons pas toujours le même avis pour certaines œuvres... Finalement, nous ne refusons pas beaucoup de pièces. Et une pièce ratée, qu'elle soit d'un bon compositeur ou d'un compositeur qui en est encore à ses débuts, a parfois son utilité : certains jeunes compositeurs ont besoin d'encouragement, ils ont besoin de mettre en relation ce qui est sur le papier, et ce qui sonne réellement.

- Quelle est votre vision de la musique actuelle, vous qui êtes en contact avec la plupart des compositeurs d'aujourd'hui?

33 Rohan de Saram : Je crois que l'un des facteurs dominants de la musique contemporaine a été le sérialisme. Mais il est possible qu'il s'agisse là du résultat final d'un certain développement de la musique européenne. Aujourd'hui, même les compositeurs de l'école de Darmstadt montrent les signes d'un changement. Il me semble que nous sommes dans un moment de réaction vis-à-vis de la musique sérielle, et qu'il tend à une sorte de "tonalité" en un sens très large. Nous jouions, par exemple, un quintette de Xenakis l'autre jour, et nous étions surpris de certaines similitudes avec la musique balinaise, dues à l'emploi de certaines gammes... mais il se peut que cela soit inconscient...

Irvine Arditti : Je crois que l'on vit dans une époque où coexistent de nombreux styles différents. Il n'est peut-être pas nécessaire d'en faire l'évaluation actuellement, mais tout simplement de jouer, d'écouter... Nous considérons que c'est notre devoir de jouer toutes sortes de musiques, et nous ne nous attendons pas à ce que chaque pièce soit un chef-d'œuvre! S'il existait une vingtaine de quatuors travaillant comme nous, nous pourrions affiner nos choix, être plus sélectifs en fonction de nos goûts... Evidemment, lorsqu'on joue la musique classique, le tri a déjà été fait, alors que pour nous, chaque œuvre nouvelle est un défi. L'interprétation peut modifier de façon considérable la perception d'une composition : nous sommes donc très liés à celle-ci. 


\title{
Stratégies picturales - le paradigme musical
}

\author{
Albert Ayme
}

1 La peinture comme elle est maintenant, promet de devenir plus subtile - plus musique et moins sculpture - enfin elle promet la couleur. (Van Gogh, Arles 1888)

2 Dès le début des années 60 je me suis attaché à mettre en place une problématique complexe et rigoureuse qui sous-entend une philosophie et implique même une éthique de la peinture, notamment par le refus du cloisonnement entre les diverses pratiques symboliques, refus qui subvertit les catégories traditionnelles de l'espace et du temps dans les rapports: intérieur/extérieur, figure/fond, peint/non-peint, additif/ soustractif, rationnel/irrationnel, réel/virtuel, l'un/le multiple, opacité/transparence.

3 Mais surtout un objectif stratégique essentiel impose mon passage à l'abstraction en 1960 : la nécessité d'une nouvelle appréhension du temps pictural qui introduit dans mon œuvre une composante musicale fondamentale incluse jusque dans ses titres mêmes ${ }^{1}$.

4 Newman, 5 variations (1988). Nécessité conjointe d'ailleurs à la prise de conscience de l'incapacité de la peinture figurative face à un tel enjeu.

5 Ces recherches sur la constitution d'un temps pictural par la couleur apportent des solutions spécifiques à des questions bien dédaignées aujourd'hui, comme celles engendrées par l'abolition de la hiérarchie dans le rapport de la figure et du fond, ou dans celui des trois couleurs primaires entre elles. Le concept même de paradigme forme le principe le plus fructueux à un problème majeur, d'autant plus fondamental qu'il est d'ordre générique et constitue l'épine dorsale de mon projet global : il consiste à penser les lois qui conditionnent les relations entre le cas particulier et le cas général qui le contient.

6 Par souci de clarté, je présenterai ces recherches sur le temps pictural à travers un parcours chronologique de mon travail.

7 D'emblée, dès juillet 1960 , je propose une première approche logique de ce problème avec la Grande Frise de 3,20 m x 1,07 m où, grâce à un format très allongé (rapport de 1 à 3), j'introduisais la temporalité par le développement latéral d'un thème rythmique qui 
était le déroulement de l'œuvre dans l'espace : sa lecture. Réponse d'ailleurs bientôt jugée insuffisante dans la mesure où mon projet définitif élargissait ses assises en négligeant les solutions ponctuelles au profit de conceptions généralisées plus objectives, impliquant une primauté des méthodes sur les fins, la conviction que les méthodes sont inclusives des œuvres.

En effet, un souci de totalité (en vue de saisir l'événement plastique dans son intégralité spatio-temporelle) joint à celui d'assurer dans l'économie maximum des moyens employés la pleine utilisation du matériau pictural, m'ont conduit à rejeter, comme inadéquate, une méthode immémoriale de juxtaposition picturale pour rechercher à travers une sorte de cristallogenèse plastique, l'élaboration d'un langage à structure transparente ${ }^{2}$.

Le principe de juxtaposition est en effet celui où l'état définitif de l'œuvre occulte, partiellement ou totalement, ses états antérieurs. Les papiers collés, découpés, superposés, relèvent sans contexte d'une telle méthode d'oblitération. Dans sa conférence d'Iéna de 1924, Klee en avait déjà révélé les insuffisances: “... les seules méthodes dont nous disposions sont discontinues dans le temps: conséquence de l'insuffisance des instruments d'expression temporelle dans le langage. Nous ne disposons pas en effet des moyens qui nous permettent de parler en termes synthétiques d'une simultanéité pluridimensionnelle." La méthode de superposition transparente est née du sentiment de ces insuffisances. Elle aspirait à donner une solution pertinente à l'interrogation de Klee. Superposition transparente, c'est-à-dire, appréhension simultanée de ces trois réalités : espace-temps-mouvement, et non plus seulement juxtaposition qui est cloisonnement de l'espace plan à deux dimensions et occultation de la temporalité véritable de l'œuvre. Le principe donnera naissance en 1962 à l'Aquarelle Monochromatique, réalisée à partir d'une unique valeur colorée. Grâce à elle, je substitue à des procédés empiriques l'application d'un principe chromatique rigoureux.

Fig. 1

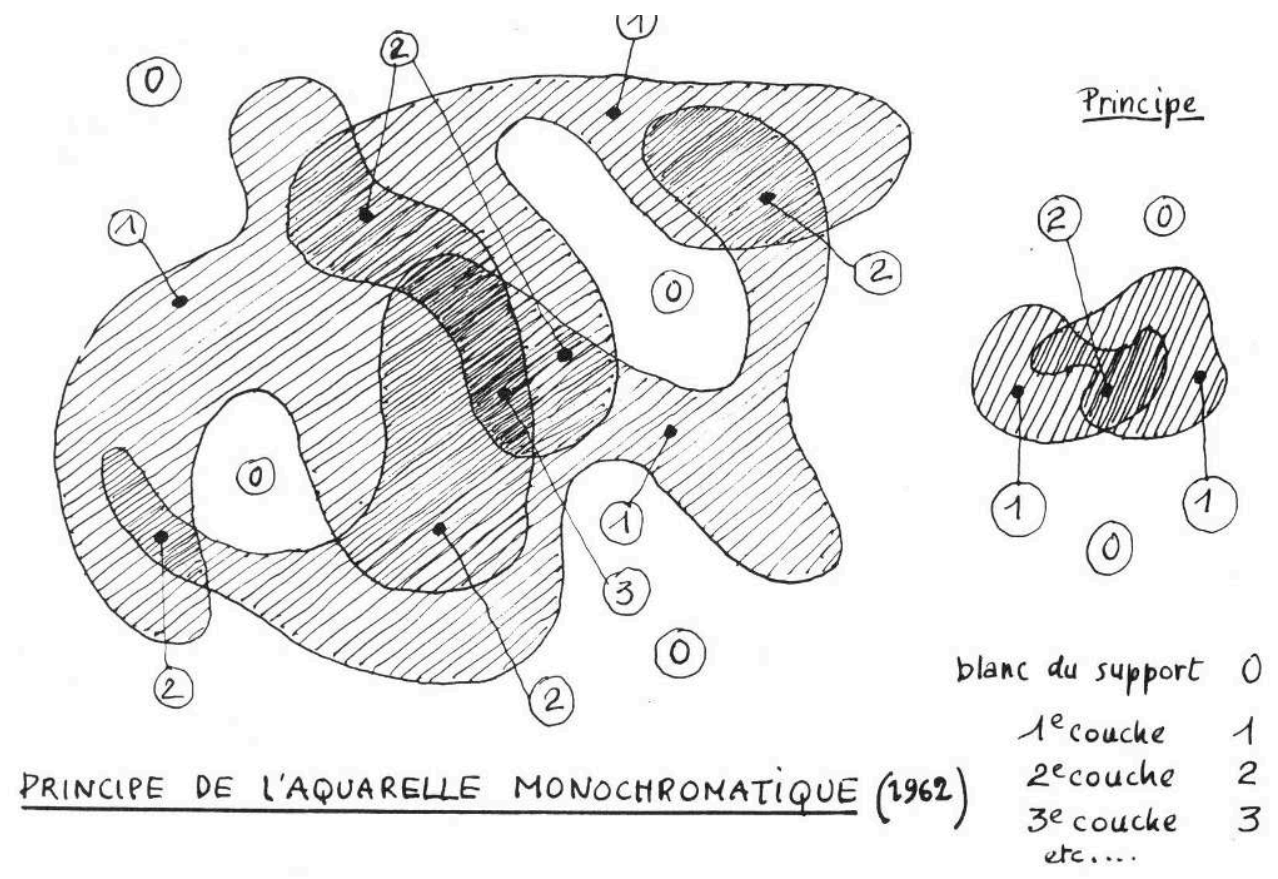


L'œuvre doit à ce principe :

- sa transparence qui rend caduque l'antinomie intérieur/extérieur, forme/fond, et qui constitue un facteur irremplaçable dans l'émancipation de la binarité ;

- une nouvelle conception de l'intervalle pictural, sans antécédent dans les arts plastiques. Cet intervalle, en effet, n'agit pas seulement sur la physique de l'œuvre. Il a une action génésique déterminante. C'est un invariant constitutif du langage. Il confère à l'œuvre une fonction harmonique. Sur le plan des valeurs tonales, couvrant un registre déterminé, le passage entre les éléments contigus de l'aquarelle s'opère mathématiquement par un intervalle égal à la valeur colorée fondamentale ou à l'une de ses harmoniques ${ }^{3}$.

11 L'œuvre est le produit de cette fusion des valeurs tonales (selon un schéma tectonique musical) et des valeur formelles (selon un processus de création irrationnelle).

Il s'agit donc en définitive d'une structure musicale irrationnelle informée qui ne se définit nullement par rapport à la musique en termes analogiques ou métaphoriques (Klee, Kandinsky...), mais en termes organiques de structure. Cette division tonale propage et condense l'espace graduellement, rythme un espace expansible dans une étendue réversible non plus seulement linéaire (diachronique), mais pluridimensionnelle (synchronique), qui module un espace transparent chromatique. Le principe chromatique engendre une structure spatio-temporelle dont les composantes transparaissent simultanément dans leur individualité propre et dans l'ensemble complexe qu'elles constituent en s'y diffusant. Cette interpénétration déploie un espace pluridimensionnel suscitant de véritables peintures polyphoniques ${ }^{4}$.

Le processus de création et son produit définitif coïncident indissolublement dans la structure transparente d'une œuvre qui déroule l'histoire intégrale de sa création (méthode pongienne). Cette conception est corroborée par l'aveu autocritique de Borges: “Je comprenais qu'il n'y a pas de mouvement hors du temps (occupation de lieux différents en des moments différents), je ne comprenais pas qu'il ne peut y avoir non plus d'immobilité (occupation d'un même lieu en des moments différents)". Conclusion avérée par l'Aquarelle Monochromatique qui consiste à enfermer le temps dans l'espace. Dans la Frise de 1960, le temps était relié à l'espace par un mouvement successif, linéaire. Dans l'Aquarelle, l'espace se déploie non seulement dans l'étendue (dimension horizontale) mais simultanément dans l'épaisseur (dimension verticale).

Dans l'Aquarelle se trouve contenu à mon insu le germe qui préfigurerait la partition dans l'espace et le temps du Paradigme du Bleu Jaune Rouge de 1976. Cette conception de base sera fondatrice de toutes les œuvres ultérieures jusqu'à la plus récente : Tombeau de Van Gogh en 1988, soit vingt-six ans après l'Aquarelle Mono chromatique de 1962.

L'Aquarelle Mono chromatique constitue donc l'étape originelle dans cette recherche de l'inscription du temps pictural.

Restait à franchir l'étape suivante, qui se révélera capitale dans l'avancée qualitative de mon projet gobai. Il s'agissait d'un problème réputé insoluble, situé dans la postérité du Carré Blanc sur Fond Blanc de Malévitch, et considéré unanimement comme une impasse, un véritable cul-de-sac. De fait, en supprimant l'unique couleur de base de l'Aquarelle, une question insensée m'était posée, une véritable aporie : comment superposer du blanc au blanc pour constituer un événement pictural? Pourtant, après combien d'insomnies, cette question sans solution trouvera la légitimité de son fondement la même année 1962, grâce à la conception et à la mise à jour du principe du Relief Soustractif, dans le prolongement économique de l'Aquarelle, qui donnera naissance au 
Monochrome Blanc, au terme de la réflexion suivante: non seulement la monochromie devient ma stricte discipline de création, mais cette monochromie bannit toute couleur autre que le blanc, lui-même absence de couleur, ou plus précisément silence optique. Dans Le Relief Soustractif, le blanc se superpose au blanc, silence sur silence, dans une opération quasi-alchimique. Dans cette œuvre, où le temps est mémorisé antérieurement à toute exploration matérielle dans les couches (de papier ou de carton) préalablement superposées, le signe de la superposition s'inverse et l'œuvre s'élabore au cours d'une superposition soustractive ${ }^{5}$.

Si l'on considère en effet l'univers plastique comme une totalité à découvrir et à épeler, l'opération additive (de l'élément isolé vers l'ensemble complexe: L'Aquarelle Monochromatique) et l'opération soustractive (de la totalité vers l'élément isolé : Le Relief Soustractif) sont des manifestations de même nature, mais de signe contraire, qui concourrent, à leur terme, mais par des trajets inverses, à la jonction des extrêmes, leur point de départ respectif : voyage entre deux néants (Kleist).

Dans Le Relief Soustractif, les composantes graphiques et morphologiques du matériau plastique sont captées simultanément, situées et datées avec précision dans l'espace et le temps de l'œuvre, étendue et profondeur réelles, effectives.

On comprend donc pourquoi les superpositions colorées dans L'Aquarelle Monochromatique trouvent dans Le Relief Soustractif leurs homologues de plans monochromes (issues de superpositions soustractives); pourquoi les harmoniques colorées se transmuent en harmoniques spatiales. Ce qui a été perdu dans l'ordre chromatique est restitué en valeurs spatiales, grâce à une concentration sévère des conditions d'application du principe primitif, nous assistons à une véritable transmutation de la couleur en espace, comme si une loi allotropique gouvernait les modalités de manifestation réciproque de ces deux valeurs, selon des critères qualitatifs inconnus.

Enfin (et ce n'est pas une moindre surprise pour l'auteur lui-même) la réserve potentielle de temps intériorisé dans l'épaisseur non seulement physique mais sémantique de l'œuvre est restituée en un mouvement quasi imperceptible mais perpétuel grâce à la lumière solaire.

21 Je précise que l'œuvre ainsi conçue n'est pas à la recherche préméditée d'un mouvement réel par une sorte de mimésis artificielle ou extrinsèque (cinétisme). Elle ne part pas arbitrairement du mouvement, elle y aboutit par surcroit.

Le Relief Soustractif se distingue donc fondamentalement du relief additif ${ }^{6}$.

Pourquoi ? Probablement parce que le problème du temps pictural n'avait jamais été posé objectivement avec une telle nécessité (Arp, papiers collés, découpés, etc.) qui est une prise analytique de la réalité plastique, une équivalence spatiale de la peinture asservie comme celle-ci au principe de juxtaposition formelle qui oblitère, partiellement ou totalement, les dimensions temporelles, et relève donc d'une conception esthétique défectible. 
Fig. 2

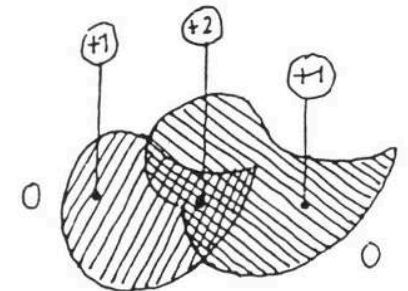

Principe

de l'aquarelle monochromatique
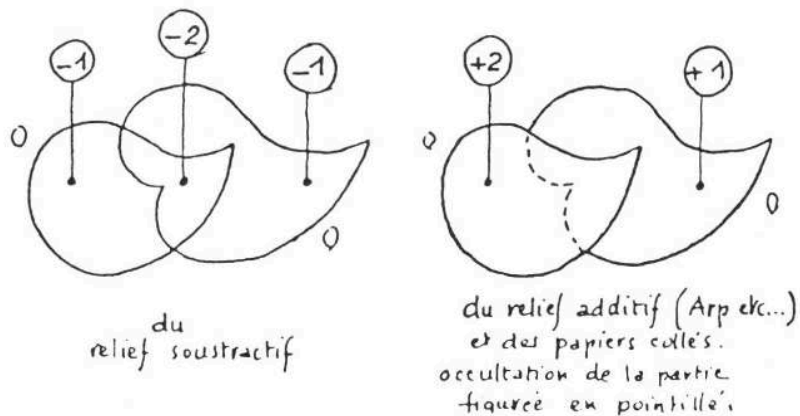

En raison de sa fidélité au principe de superposition transparente, Le Relief Soustractif assure sa continuité graphique grâce à une interpénétration des plans à travers les multiples niveaux successifs de l'œuvre : c'est une création plurilectique.

Ces deux phases initiales de mon travail sont fondamentales. Elles constituent les assises intangibles sur lesquelles tout l'édifice de mon projet global prendra son appui, jusqu'à l'époque la plus récente.

6 En 1963 j'abordais un problème d'une grande complexité à travers Seize et une Variations, une œuvre multiforme qui développe un thème plastique soumis à la loi sévère de la fugue : travail à partir d'une unique forme "trouvée", non-géométrique, au terme duquel est ressuscitée, dans une double fugue formant la dix-septième et ultime variation, la forme originaire, inversée, non-peinte, dans un mouvement à structure circulaire. Oeuvre historiquement importante et qui se révèle comme le lieu d'émergence du concept même de paradigme dans mon itinéraire pictural, ce titre Paradigme lui ayant été primitivement attribué, puis avec raison, retiré.

A travers cette grande fugue picturale s'entrecroisent en réalité trois hommages : à un écrivain, à un musicien et à un peintre. Double rappel implicite d'abord d'un travail d'écriture antérieur (Raymond Roussel), l'œuvre de celui-ci faisant référence à l'Oratorio Vesper de Haendel construit selon un procédé fourni par le hasard seul et consistant à se faire dicter une note à chacune des 23 marches d'un escalier descendu par le musicien ${ }^{7}$.

Enfin, à l'intérieur des Seize et une Variations est inséré avec la $15^{\mathrm{e}}$ variation un hommage non préconçu à Uccello. L'ensemble constituant une chaîne d'interrelations en profondeur (pratique de la mise en abîme). On s'aperçoit alors que les rapports d'homologie structurelle de cette œuvre avec la musique apparaissent avec une grande pertinence. Nous sommes ici en face d'une démarche historique non exclusive de la peinture et fondée sur un refus $d u$ cloisonnement entre les diverses activités symboliques en vue de la reconnaissance tacite d'un statut unique pour toutes les méthodes de création poétique $^{8}$. On peut retrouver cette volonté dans les œuvres de quelques grands écrivains contemporains (Francis Ponge, Michel Butor, Roger Laporte...).

Oeuvre fuguée donc, formée de seize fugues, plus une (la forme d'origine et la double fugue ultime qui se superposent en s'annulant). Oeuvre cyclique, mais non hermétiquement close cependant, car la seizième variation brise le schéma de détermination concertée de l'ensemble en éveillant les virtualités illimitées de l'ordre irrationnel (éclatement et pulvérisation de la forme). Démarche logique où le sujet de l'œuvre prend son origine dans le matériau plastique lui-même, où genèse et état 
définitif sont confondus dans la structure transparente d'une œuvre qui révèle en filigrane l'histoire de sa création. Aperception globale d'un espace multiple discontinu ramené à son unité organique de conception, c'est-à-dire envisagé comme un tout indivisible, multiple et unique simultanément (déploiement en album, reploiement en livre : Mallarmé). Oeuvre, de plus, articulée selon une syntaxe corrélative de la loi de superposition transparente qui lui est implicite (explicite dans L'Aquarelle Monochromatique). Cette tentative montre la connexité du temps et de l'espace dans l'univers plastique puisque l'introduction d'une temporalité inusitée multiplie les dimensions traditionnelles de l'espace pictural.

Jusqu'ici j'évoluais dans l'abstraction à l'intérieur d'un langage irrationnel, nongéométrique. Le passage en 1965 au vocabulaire géométrique (et le recours au carré comme figure neutre impersonnelle, tombée depuis plus d'un demi-siècle dans le domaine public) m'a été imposé par des exigences objectives intrinsèques à l'abstraction et non pour servir des préférences personnelles. Lorsque le vocabulaire cesse de se personnaliser, l'action se porte pleinement sur les processus de création et les phénomènes picturaux qu'ils sollicitent. Ce choix décisif a surtout conditionné l'émergence d'une démarche paradigmatique dans le prolongement des Seize et une Variations. A L'Hommage à Malévitch de 1967, qui comprend seize variations fondées sur le carré et sur l'application du principe du Relief Soustractif, succède enfin, au terme d'une longue gestation (dix ans, 1964-1974, pour "poser les règles de bonne formation") l'œuvre longtemps "rêvée" et préparée intérieurement : Paradigme.

31 Elaborée à partir d'un unique invariant, le carré de base, cette œuvre combinatoire à croissance illimitée (que je ne puis ici schématiser) se caractérise par ses lois propres : c'est la méthode mise en œuvre pour faire fonctionner correctement l'élément formel qui est importante et non celui-ci. Non comme répétition formelle d'un modèle de base, mais articulée selon la notion de différence (variations illimitées des plus petites différences des possibilités génératrices de ce modèle).

32 Avec Paradigme en 1974, puis Paradigme 2 en 1982, je me trouve enfin engagé au cœur même de mon projet global, clé de voûte qui a supporté mentalement depuis l'origine ma recherche picturale, et qui me semble de grande portée en ce qu'elle est sans antécédent dans les arts plastiques par l'extrême concentration de ses moyens et le contrôle maximum exercé sur ses paramètres.

33 Les enjeux historiques de Paradigme 2 sont donc considérables à travers la mise à l'épreuve pluri-lectique simultanée de tous les les signifiants picturaux sollicités à travers les rapports: support/forme, plus/moins, peint/non-peint, inscription/ extraction, réel/virtuel, aplat/découpe, rationnel/irrationnel, opacité/transparence, noir/gris, noir/blanc, blanc/gris, l'un/le multiple.

A partir d'un unique carré de base, ce projet se fonde sur trois concepts fondamentaux : le peint $A$, le non-peint $B$ et la parité entre peint et non-peint C (le damier), soit qui correspondent respectivement à des opérations additives, soustractives et d'équivalence. Le jeu de ces trois occurrences de base se manifeste picturalement selon les trois couleurs de la gamme a-chromatique : blanc/gris/noir. Le dispositif opératoire ordonne trois ensembles ternaires (triades) constituant la matrice d'engendrement multipolaire: matrice telle que toute hiérarchie est abolie sans équivoque dans ce système entièrement ternaire. Son caractère de réversibilité, de permutabilité parfaite entre les composants, apparaitra de première importance dans la rencontre non préméditée avec le Carré Magique en 1983. 

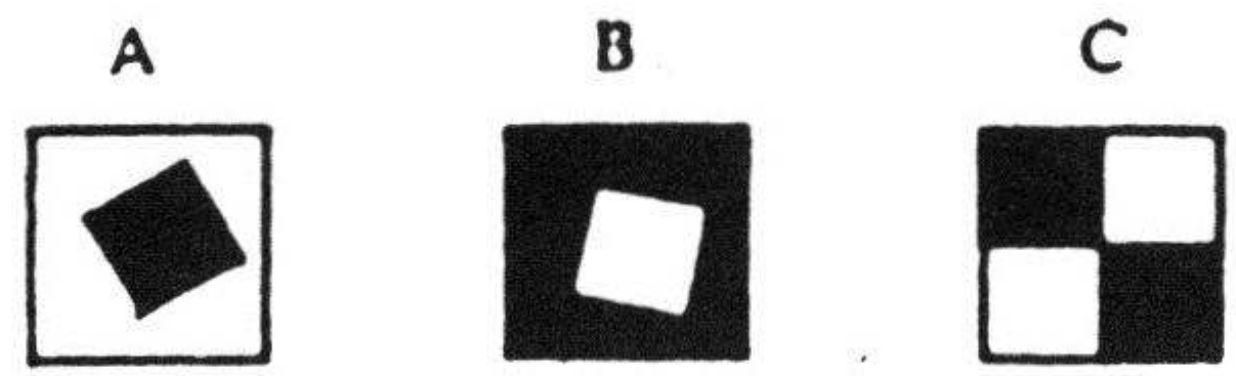

Fig. 4

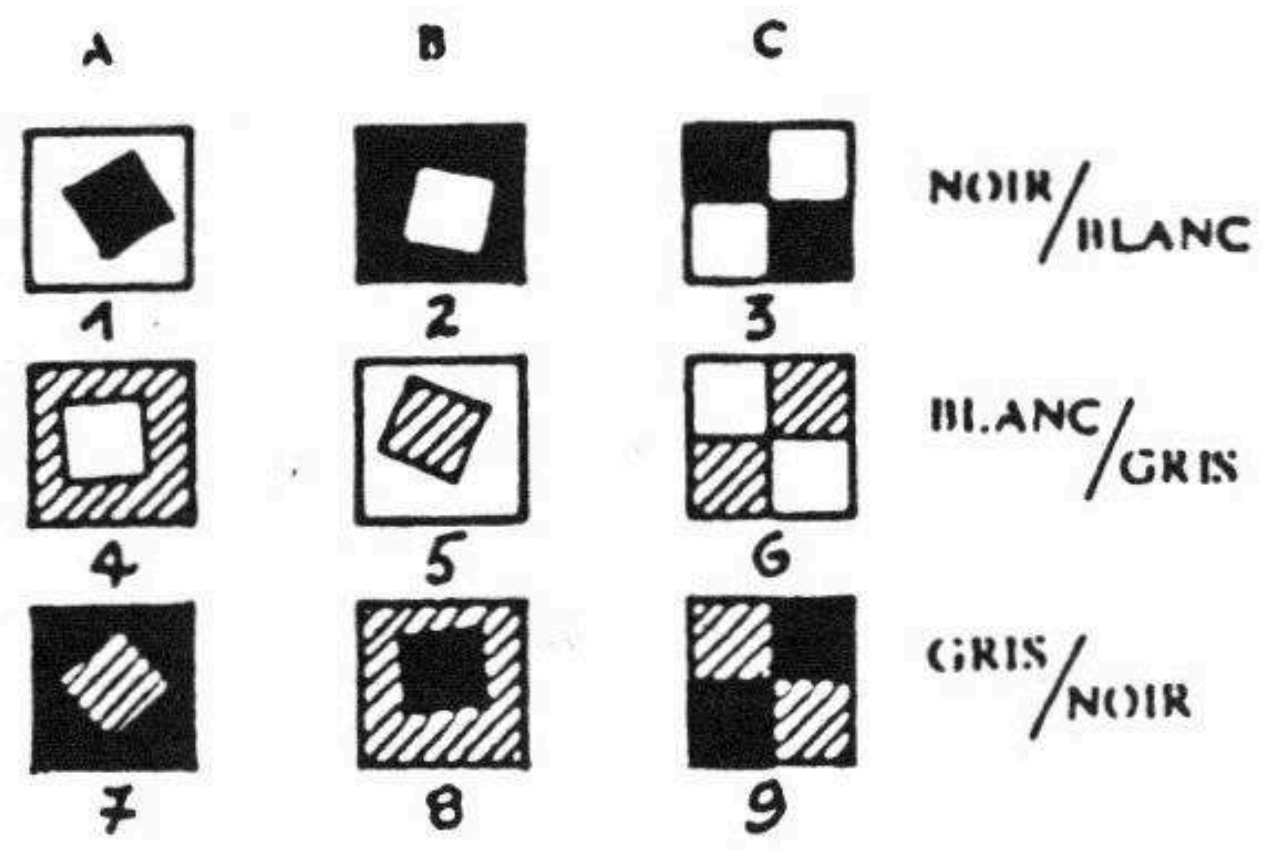

Fig. 5
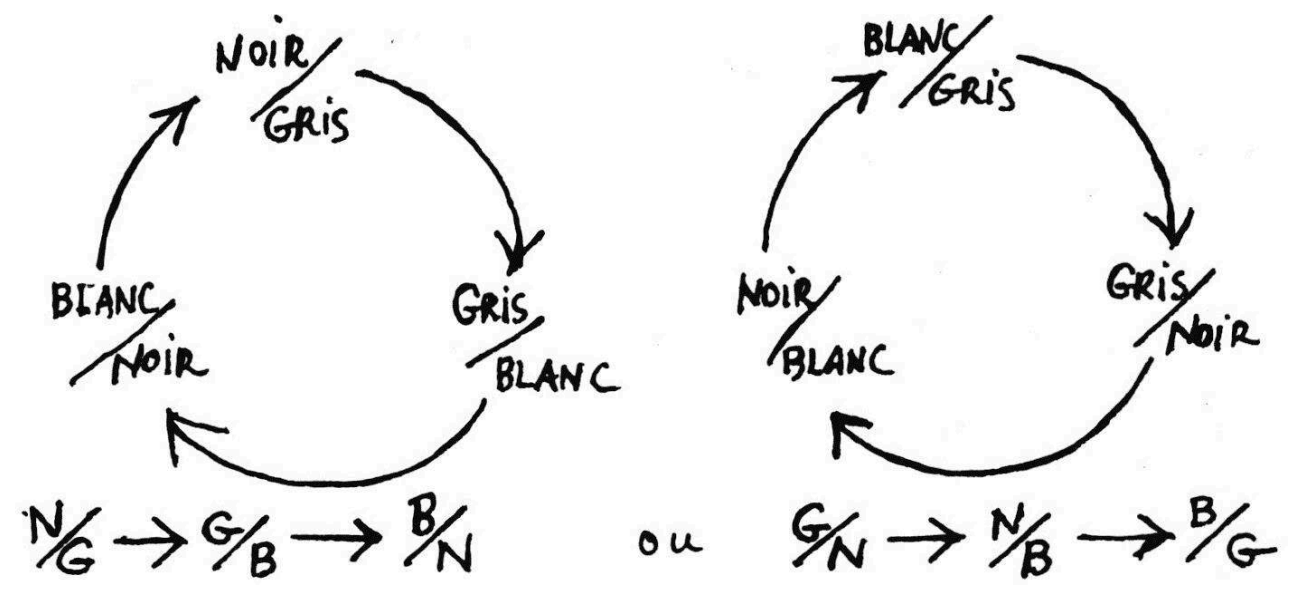
Cette matrice me permet d'établir une vaste combinatoire entre les 9 termes commutatifs de base de la matrice, qui engendre aussi 729 séquences de 3 termes

Fig. 6

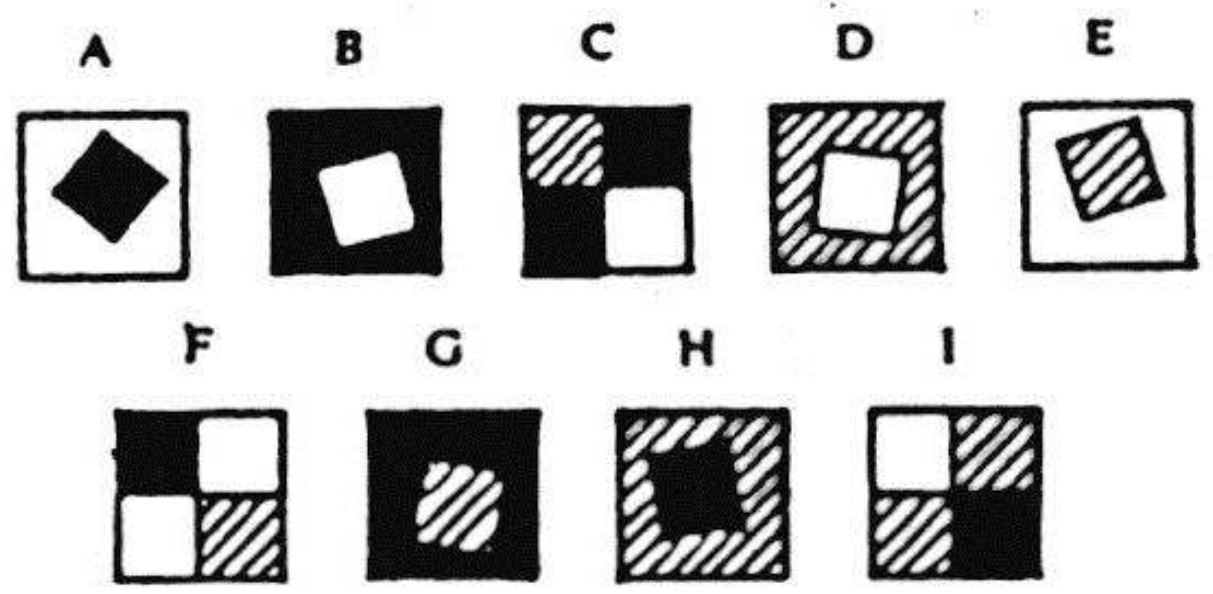

36 quelques exemples :

Fig. 7

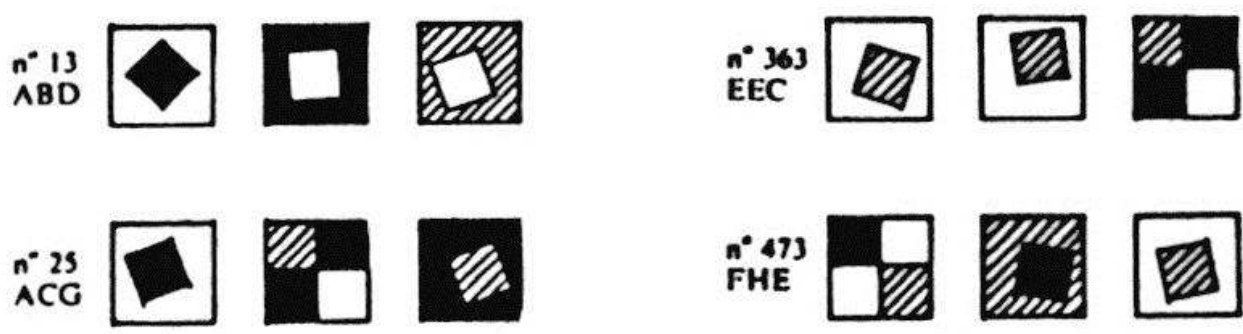

37 chaque séquence de 3 termes superposés subit l'opération de superposition soustractive grâce à la double découpe du carré de base (double mise en abîme)

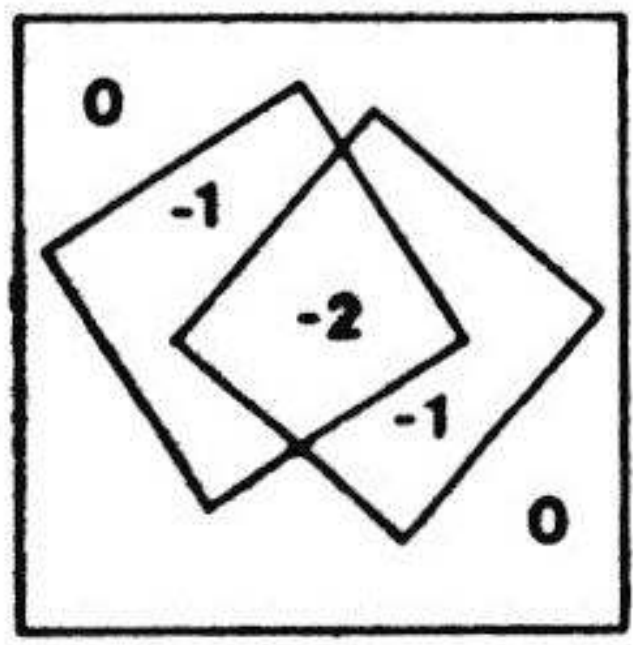

Paradigme 2 est mon œuvre clé. Elle rompt avec la conception de "la série" qui la précède (celle de Monet par exemple) ${ }^{9}$. 
39 La série idéale pourrait se concevoir sous la forme d'une circonférence, car c'est un ensemble fermé, organiquement limité par la rétention de la loi entropique du genre, tandis que Paradigme 2 serait représenté par une spirale sans fin.

Fig. 8

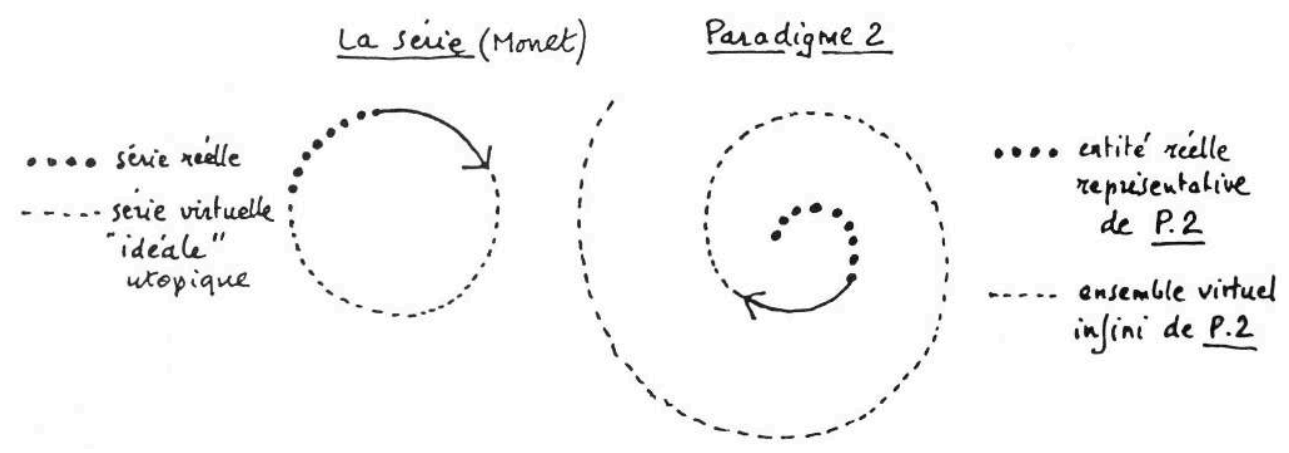

On pourrait dire que la puissance génératrice de Paradigme 2 émane d'un processus centrifuge, tandis que le mouvement de la "série" se caractérise par sa tendance centripète.

Les enjeux qui sont visés simultanément à travers cette œuvre sont considérables, que ce soit par l'abolition des hiérarchies et à travers les rapports de l'un et du multiple, du réel et du virtuel ou du subjectif et de l'objectif. A partir d'une telle œuvre, explorant une voie nouvelle, s'opère une mutation qui rompt avec la conception, la forme et le code de lecture des œuvres traditionnelles. Paradigme 2 est le lieu d'une confrontation fructueuse entre tous les signifiants picturaux mis en œuvre. Opérant un renversement entre une démarche logique et ses incidences qui se caractérisent par une "irrationnalité objective", Paradigme 2 est assimilable à une véritable machinerie investissant un lieu mental et physique entièrement mobile où l'individuel anticipe sur le générique qui le contient. Ainsi, l'aléatoire intrinsèque au "sujet pictural", limitatif par essence, est transféré, puissance $n$, à la combinatoire, dépositaire impersonnelle du principe de non-intervention préméditée de l'auteur : passage du subjectif à l'objectif. Dès ses préalables, le dessein avoué de Paradigme 2 se trouve donc exaucé par cette nécessité de créer, en amont, les conditions objectives de non-intervention préméditée de l'auteur. Car le véritable problème que traite mon œuvre, son dessein éthique, se situe en amont, au cœur même de sa conception: comme celle d'un fleuve la source d'une œuvre est son point culminant. C'est comprendre que son statut, le principe de son jeu de postulats, d'invariants et de règles qui gouvernent son économie interne, constitue une valeur et une réalité immanente. Position confortée par Anton Webern évoquant la genèse de ses Six Bagatelles pour quatuor à cordes, op. 9: "J'ai eu le sentiment qu'une fois que les douze sons étaient apparus, le morceau était terminé". Ainsi de Paradigme 2 où dans cette recherche des lois fondamentales le cas général comprend des cas particuliers. La réserve de savoir concentrée dans Paradigme 2 est infinie : la structure même de cette œuvre la prédestine à un inachèvement consubstanciel, aucun terme actualisé ou potentiel n'étant récusable.

Paradigme 2 constitue l'archétype d'une œuvre générique sans modèle antérieur, un modèle possible d'œuvre combinatoire à croissance illimitée.

Toutes ces œuvres depuis 1960 ont limité au strict minimum la participation de la couleur (les recours aux principes monochromatiques, monochromiques et à la gamme 
a-chromatique du noir et blanc ayant révélé leur pertinence). Mais la raison principale vient $\mathrm{du}$ fait qu'il s'agissait pour moi de recherche fondamentale, d'une expérimentation attachée essentiellement à la mise au jour de méthodes et de structures spatio-temporelles inédites, dans lesquelles la couleur n'est pas impliquée au premier chef.

Mais un changement radical intervient à compter de 1975. Désormais la couleur va prendre se place prééminente en tant que signifiant exclusif, d'abord dans le Paradigme du Bleu et Jaune, puis du Bleu et Rouge, enfin et surtout dans le Paradigme du Bleu Jaune Rouge de 1976. Je rappelle donc ici mes positions prises tout au long de mon itinéraire plastique: mon propos est d'ordre logique, non psychologique ou esthétique. Il concerne donc le langage pictural, plus précisément sa syntaxe, et se traduit par des conséquences importantes dans ce maniement de la couleur elle-même. D'abord, en faisant de la couleur le moyen et la fin du "tableau", se trouve proscrit le recours à tout signifié extra-pictural, et ipso facto à la peinture figurative tout entière. Mais encore cet art autonome n'empruntant qu'à lui-même et "libéré des fonctions du discours" (Lyotard) exclut de son champ d'exploration l'emploi de la couleur comme un adjuvant ou comme repère, marquage invariable, signal monochrome.

A cette citation de Braque rappelée par Francis Ponge - "L'objet, c'est la poétique" - je réplique : la poétique, c'est la couleur. Car le maintien intangible du spécifique par la couleur seule me semble une exigence sans laquelle se perpétuent les anciennes catégories hiérarchiques, les vieilleries picturales. Lorsque je parle du traitement de la couleur, celui-ci implique donc un mouvement, un fonctionnement, un travail de (et sur) la couleur, analogue à celui pratiqué sur la langue même: Rimbaud, Mallarmé, Ponge...

Ces prises de positions radicales me situent d'emblée hors d'un système de rapports de couleurs par juxtaposition dont Matisse peut être désigné comme le représentant le plus éminent (et Barnett Newman, me semble-t-il, comme son équivalent dans l'abstraction, un grand nombre de peintres abstraits m'apparaissent comme des figuratifs embusqués). Mais dans ce système de rapports, la peinture n'engendre le plus souvent qu'un traitement spatial, bi-dimensionnel de la couleur, séquelle de la fameuse déclaration de Maurice Denis: "Avant d'être un cheval de bataille ou une femme nue, une peinture est une surface plane recouverte de couleurs en un certain ordre rassemblé"10, qui contient le germe de l'hédonisme et du formalisme. Autre critère distinctif de ma démarche : bien sûr, "quelque chose à obtenir et non plus quelque chose à exprimer" (Francis Ponge) mais encore : non plus "quelque chose à obtenir" par des pratiques empiriques voire anarchiques de composition soumises au primat de la subjectivité, mais comprises dans un projet global qui, excédant le pur rétinien ${ }^{11}$, suscite l'entendement et résulte d'options fondamentales qui constituent le socle sur lequel est ancrée ma démarche picturale :

1. Le choix des couleurs réduit à ses trois couleurs fondamentales et irréductibles: les trois primaires Bleu Jaune Rouge exclusivement.

2. Le refus de tout mélange entre ces trois couleurs superposéees en transparence. Critère capital : en refusant le mélange, s'affirme le principe de la discontinuité. La notion de transparence transcendant les dichotomies intérieur/extérieur, forme/fond. Mélanger les couleurs, c'est faire valoir leur continuité, c'est se référer au cercle chromatique des écoles de peinture (le Bauhaus, l'Impressionnisme, Klee, Matisse...) une conception du "continuum" ininterrompu du prisme coloré de la lumière. Le principe de discontinuité 
chromatique n'est pas, dans mon cas, appliqué en priorité à l'espace (Klee, Delaunay) mais au temps. Distinction capitale puisque "avec les superpositions transparentes des couleurs le temps cesse d'être un effet de l'œuvre sur un destinataire, il est inclus en elle". (J. F. Lyotard).

3. L'abolition de la hiérarchie entre les trois primaires, elle permet de se soustraire au cadre de référence du système ancestral de rapports de couleurs par juxtaposition: pratiques empiriques de la couleur. La suppression de tout privilège discriminatoire entre les trois primaires est assurée grâce à l'innovation d'une méthode de tressage dans laquelle, à travers leurs multiples trajets, chacune des trois couleurs primaires est tressée, à son tour, dans l'une des trois positions : dominante, médiane, dominée, selon un ensemble de combinaisons possibles :

Fig. 9

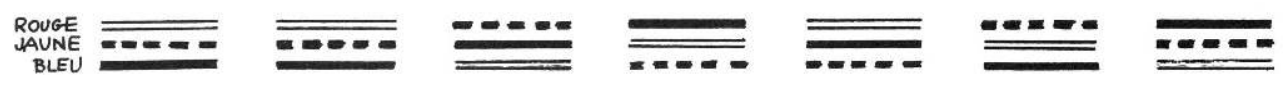

Sorte de coupe géologique dans l'épaisseur des œuvres et qui révèle les strates précises de la couleur.

51 4. Ce principe de tressage des trois primaires doit être impérativement mis en œuvre selon une technique adéquate du maniement de la couleur. A la méthode de juxtaposition des couleurs se substitue donc une méthode de superposition transparente à travers une technique de progression de la couleur dite "en canon" s'apparentant aux voix et qui contribue à l'élaboration d'une technique musicale de la peinture. Cette caractéristique apparait pleinement avec la création, depuis Seize et une Variation en 1963, d'œuvres discontinues à multiples variations. Enfin l'application technique du tressage par superposition transparente des trois primaires exige le choix approprié d'un médium (teinture transparente) et d'un support (toile de coton non préparée) et répugne à tous les tripotages de la facture, grâce à l'impersonnalité d'une frappe franche et directe de la couleur au pochoir. Ce sera, en 1975 et en 1976, la mise au jour de Paradigme du Bleu Jaune Rouge (voir ci-dessous la reproduction de cete œuvre, ainsi que le diagramme de lecture indiquant le tressage des couleurs) dans lequel la couleur fonctionne comme une partition dans l'espace et le temps (horizontalité et verticalité simultanées) ${ }^{12}$.

Tous ces critères de conception et leurs enjeux périment des pratiques empiriques et analytiques de composition au profit "d'un type général de recherches" et de méthodes menées sur des bases plus objectives dont l'homologie est évidente, et pour des ambitions identiques à travers le temps, avec le postulat et les techniques de la musique dodécaphonique dans le strict respect de leur matériau spécifique ${ }^{13}$.

Ecoutons Webern : "Les douze sons sont sur un pied d'égalité. Si l'un d'entre eux était répété avant que les onze autres ne soient apparus, cela lui conférerait un certain privilège... Nous avons éprouvé le besoin de faire en sorte qu'aucun son ne devienne prédominant et ne puisse, en étant répété, prendre l'avantage sur les autres."

Dans son principe, c'est précisément le but poursuivi grâce au tressage des trois couleurs primaires pour supprimer tout privilège discriminatoire entre elles.

On pourrait suggérer que le système pictural, depuis l'impressionnisme particulièrement, est fondé en grande partie, par analogie avec le langage musical, sur un concept mélodique (la dimension horizontale) tandis que les caractéristiques 
inhérentes à ce système nouveau conjuguent simultanément les dimensions horizontales (mélodie) et verticale (harmonie). La pluridimensionnalité qui en émane engendre un langage pictural polyphonique dans lequel temps - espace - couleur, indissociable, constitue une seule et même chose, une entité indivise ${ }^{14}$.

Rattaché au postulat de Maurice Denis, le système de référence matissien conduit à un assemblage spatial. Modifier si peu que ce soit cet assemblage détruit la composition du tableau, ainsi que Matisse l'écrit très justement. Ses papiers découpés portent à son état d'extrême acuité cette logique des rapports de couleurs en accusant d'autant plus clairement leur nature spatiale.

$\mathrm{Au}$ contraire, impossibilité absolue de concevoir une quelconque intervention sur la structure spatio-temporelle du Paradigme du Bleu Jaune Rouge, où les trois primaires tressées entre elles indissolublement par superpositions transparentes s'interpénètrent et forment un tout intangible, interdisant même tout repentir. C'est donc en ce point précis que se déterminent la spécificité et l'ambition de mon projet global relatif à la couleur et, me semble-t-il, sa portée historique, qui ne trouve nullement sa source, ou sa justification, ni dans sa conception ni dans ses techniques, dans la théorie des couleurs issue de Chevreul, et encore moins dans son application par Delacroix et les Impressionnistes ${ }^{15}$. L'introduction de ces exigences nouvelles est commandée essentiellement, il faut le répéter, par l'inscription du temps pictural, mais d'un temps pictural matériellement constitué par la couleur, et non d'un temps métaphorique surimposé après coup sur une œuvre par l'imagination du peintre ou du critique d'art. Car la peinture sécrète sa propre morale implacable dans le "faire" justement, qui affirme, en dernier recours, sa suprématie sur tout discours postérieur. "Dire ce que l'on fait, faire ce que l'on dit" (Francis Ponge).

Exigences qui marquent la rupture profonde avec le système existant, notamment au sein même de la syntaxe picturale fortement subvertie par l'adoption de structures spatiotemporelles et rythmiques où les trois primaires, superposées en transparence, se transmuent d'abord en leur trois complémentaires, puis en leurs six bruns. L'application de ce principe chromatique rigoureux et le recours exclusif à la transparence constituant, rappelons-le, un facteur capital dans l'émancipation de la binarité ${ }^{16}$.

Ensemble de conditions dont l'adéquation conduit en 1975 à la naissance du Paradigme du Bleu et Jaune, puis l'année suivante, à celle de Paradigme du Bleu Jaune Rouge où ces notions sont mises en pratiques. Dans cette dernière œuvre notamment et dans une conception élargie de Seize et une Variations de 1963, les problèmes de composition ne ressortissent plus à des solutions ponctuelles apportées au coup par coup, mais sont inclus, absorbés dans un système plus vaste qui convoque, au lieu de la pièce unique (le chef-d'œuvre), la notion de "constellation" (constellation d'œuvres) que celle-ci soit sollicitée à travers une œuvre finie et autonome (Seize et une Variations 1963, Blason d'un Peintre 1980, Triple suite en Jaune à la gloire de Van Gogh 1982-1988, Tombeau de Van Gogh 1988) ou à croissance illimitée (Paradigme 2 1982) ou bien encore à variations multiples indéterminées (Paradigme du Bleu Jaune Rouge 1976, Le Carré Magique 1983). Dans ces trois cas de figure il s'agit de tentatives de réponse de type générique. Avec, plus particulièrement, le cas limite de Paradigme 2, où les trois couleurs de la gamme achromatique (gris, noir, blanc) substituées aux trois couleurs primaires, ont permis l'accession à une solution de nature combinatoire à croissance illimitée, qui se caractérise par son infinitude. 

d'équivalent, me semble-t-il, que dans la musique, et qui apparaît comme le noyau générateur de toute ma démarche picturale, j'ai cherché à occuper la place virtuelle du peintre générique. Peut-être faudrait-il voir dans cette ambition l'esquisse d'un mouvement de transition vers une conscience baroque, c'est-à-dire de l'individuel autonome à l'ensemble infini. Passage d'une structure spatiale statique à une matrice multipolaire ouverte et mobile. Du cercle (la série de Monet) à la spirale (Paradigme).

61 Restitution des œuvres donc, à l'univers de la mobilité; lecture se décomposant en une suite d'opérations déroulées dans le temps à travers les multiples trajets des trois primaires s'interpénétrant par tressage dans l'épaisseur du support. Par cette propriété caractéristique, cette démarche échappe à toute annexion par l'école dite géométrique ou constructiviste assujettie à l'inertie de composantes vouées aux valeurs exclusivement spatiales de la peinture. Elle requiert, au contraire, de re-découvrir, sous-jacents à la surface visible, tous les trajets entrelacés des trois couleurs dans l'épaisseur de l'œuvre dont la structure stratifiée est comme traversée par l'ébranlement rythmique de la couleur.

Ce travail ne se propose donc pas comme la recognition d'une géométrie interne de la nature chargée de reconstituer des figures géométriques préexistantes, mais comme la mise en œuvre des moyens aptes à créer les conditions d'apparition des lignes de tension de la couleur. Processus d'élaboration d'une structure par la couleur donc, et non d'une figure par un dessin surimposé (la couleur dessine).

Quoi qu'il en soit, tous ces cycles d'œuvres diversifiées répartis sur un registre de grande envergure mettent à l'épreuve cette poétique de l'unité qui reste pour moi la règle d'or de ma pratique picturale : variété obtenue à partir de l'unité, qui est aussi un principe musical. Qui perd la musique perd la voix (la voie...)

Précisément il est devenu bien nécessaire de clarifier ce problème des rapports de la peinture avec la musique. C'est à compter du Paradigme du Bleu Jaune Rouge et de ses acquis, enrichis dans la Triple suite en jaune à la gloire de Van Gogh et dans le Tombeau de Van Gogh, dont la cinquième et dernière Station se clôt par un geste musical d'envergure : une double mise en abîme conjuguée avec une inversion en miroir (voir ci-dessous la page de travail manuscrite de cette $5^{\text {e }}$ Station). Certes, les liens entre peinture et musique n'ont cessé de s'entrecroiser, mais leurs rapports continuent, indûment, de s'établir en termes de consonances, associations, réminiscences, confondus de la sorte avec musicalité, qualité subjective commune à de nombreuses peintures et qui résulte d'analogies externes, sensorielles. Dans le même état d'esprit, les impressionnistes étaient plus sensibles à l'harmonie des couleurs du tableau qu'à sa structure, en oubliant que celle-ci détermine celle-là. Ce qui est un leurre dont, curieusement, les textes et les recherches de Kandinsky (et dans une moindre mesure ceux de Klee ${ }^{18}$ ), entretiennent l'équivoque en laissant le problème irrésolu. C'est en récusant toute référence analogique ou métaphorique que les rapports de mon travail avec la musique, fondés sur une fonction organique, se conçoivent exclusivement en termes de structures, et comme tels, s'ils suscitent des équivalences, c'est toujours sur le plan méthodologique et dans le strict respect de leur spécificité propre, à l'exclusion de tout amalgame.

Plus précisément encore, dans cette tentative d'inscription du temps pictural qui est la mienne, "le temps cesse d'être un effet de l'œuvre sur le destinataire, il est inclus en elle" (Lyotard). Cette inclusion est donc intrinsèque au matériau pictural: c'est par la création de structures spatio-temporelles spécifiques que se révèlent les homologies 
foncières par où les deux arts communiquent, bien moins, si j'ose dire, au regard de leurs fleurs que de leurs racines... Primauté du processus générateur (l'infrastructure latente de la partition) sur l'effet de surface (manifeste), celui-ci résultant de celui-là. Pour me permettre d'être subtil, il me faut un outil puissant. La nuance naît de la force, non de l'insuffisance.

C'est dans cet esprit, notamment, que se conçoit le Paradigme, telle une immense fugue virtuelle, un polyptyque sans fin: une seule gamme mais qui emplisse tout l'univers. Ainsi se justifie cette entreprise qui se propose d'unir, de réconcilier si faire se peut, l'homme visuel et l'homme auditif, coupés si souvent l'un de l'autre.

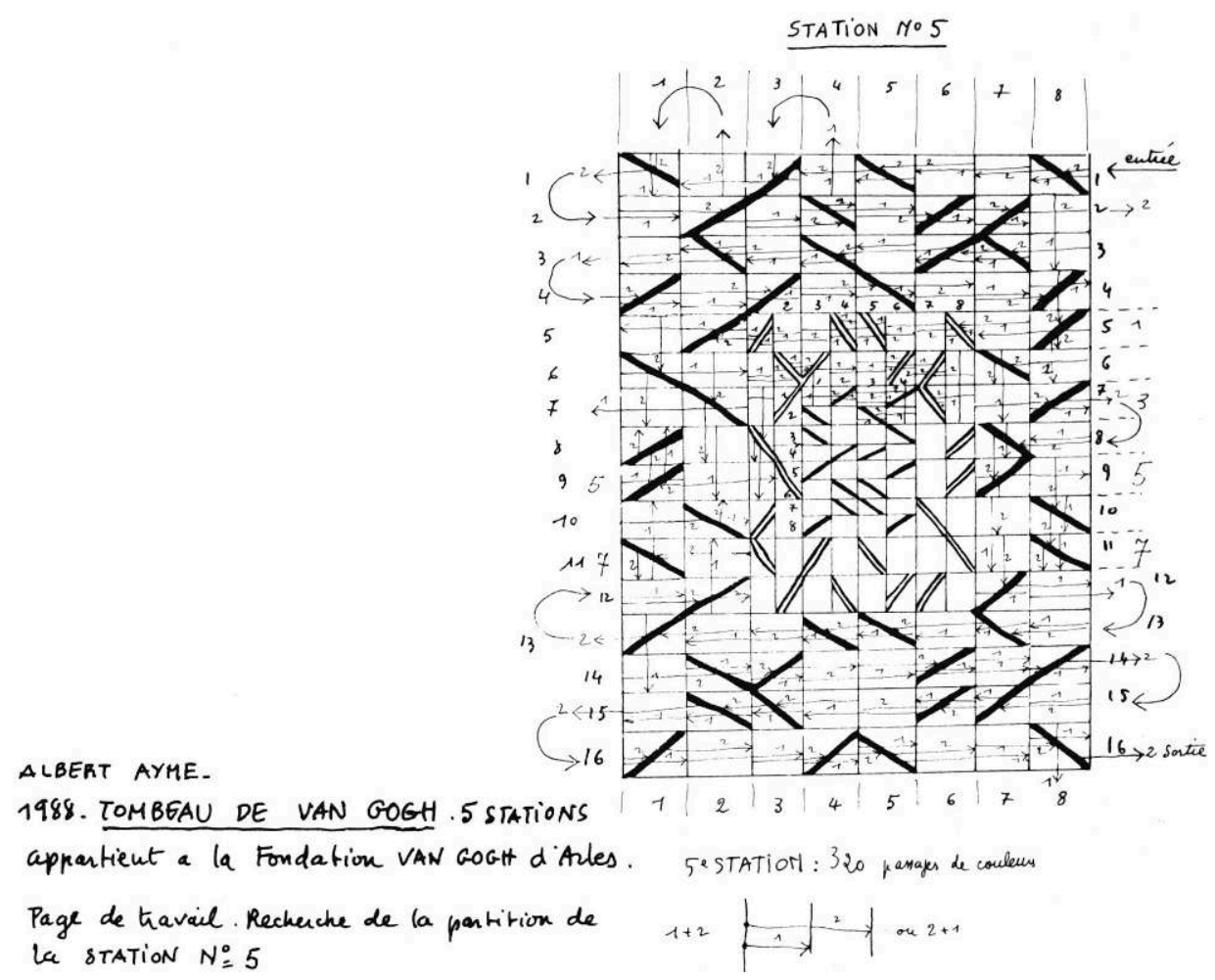

Pour apprécier le travail pictural de Albert Ayme impliqué dans son texte, on peut se reporter à l'ouvrage suivant: Ayme, Albert: Triple suite en jaune à la gloire de Van Gogh, avec des textes de Michel Butor et Michel Sicard, Traversière, Paris, 1987 (nombreuses reproductions couleurs de l'œuvre).

\section{NOTES}

1. Seize et une variations (1963), Mille monochromes blancs (1967), Paradigme (1974), Paradigme 2 (1983), Suite des trois mémoires (1979), Paradigme du Bleu Jaune Rouge (1976), Blason d'un peintre (1980), Triple suite en Jaune à la gloire de Van Gogh (1981-86), Tombeau de Van Gogh, 5 stations (1988), Quintette pour B.

2. "La structure transparente est celle où profondeur et surface se montrent ensemble à notre regard" (Ernst Jünger). 
3. Aux musiciens d'établir les homologies possibles avec le système dodécaphonique, dans lequel les douze sons diffèrent entre eux par un intervalle invariable d'un demi-ton, mais irrationnellement en ce qui concerne les valeurs formelles (organisation de l'espace de l'œuvre selon une trame irrationnelle).

4. "La peinture polyphonique est supérieure à la musique en ce sens que l'élément temporel y est plutôt une donnée spatiale. La notion de simultanéité (transparence) y apparaît plus riche encore" (Klee).

5. Si les procédés additifs peuvent dans une certaine mesure être qualifiés de "physiques", nous pouvons qualifier les systèmes soustractifs de "chimiques".

6. Bien des années après, je découvris, stupéfait, que le principe du relief soustractif n'existait dans aucune civilisation, fût-ce la plus reculée...

7. Cette œuvre de Haendel est le fruit de l'imagination de Roussel.

8. Avec celle de Ponge, deux réactions importantes à cette œuvre: Barthes (en 1963) : “Admirable leçon structurale dont je me servirai certainement" et Boulez (en 1964, depuis Baden-Baden) : "J'ai été très sensible à la direction qu'il propose, où je peux en effet retrouver mes propres préoccupations".

9. “C'est dans cette voie, écrit Jean Ricardou, que Claude Monet par exemple se risque avec la série des Cathédrales de Rouen où, stabilisant un certain nombre de paramètres, le format, le support, la peinture, l'objet représenté, le point de vue, il multiplie les variations de la couleur. C'est dans cette voie que A. A. accomplit un saut décisif. L'artiste a su tirer les prodigieuses conséquences de la désacralisation de l'invariant de base. La cathédrale de Rouen chez Monet est non seulement un objet à représenter, mais encore, par elle-même, déjà une œuvre d'art. A ce double titre, son opérabilité est doublement restreinte. Le carré, chez Ayme, permet de sortir simultanément de la surveillance de la représentation et de la déférence artistique ou de son envers complice, l'iconoclastie, vis-à-vis de l'invariant." cf. Colloque de Cerisy-la-Salle, 17-24 août 1982 : Albert Ayme et le paradigme en peinture.

10. Système d'assemblage des couleurs auquel sont également assujettis la plupart des peintres abstraits.

11. Au sens où Cézanne dit que "La réflexion modifie la vision".

12. Suite inattendue des Grands Draps muraux de 1962 non-montés sur châssis, première manifestation de la "toile libre" dans le monde. Les Grands Draps muraux sont basés sur une dialectique du peint et du non-peint.

13. La gamme de base des douze couleurs résultant

- des trois primaires,

- des trois complémentaires,

- des six bruns,

n'est pas sans analogie avec la série des douze sons.

14. De même que Ponge écrit : "Le sens c'est la forme et la forme c'est le fond".

15. Au contraire de ce qu'a écrit Georges Roque dans : Deux sources de la couleur française, in Art Press, $\mathrm{n}^{\circ} 128$.

16. Comme toutes mes œuvres d'ailleurs s'émancipant de la binarité depuis 1962 (principe du Relief Soustractif).

17. Binarité que Y.-A. Bois considérait comme irréductible dans des propos sur Matisse, à tort au regard de l'abstraction...

18. Kandinsky et Klee n'auraient-ils pas inconsciemment été inhibés par leur propre pratique instrumentale de la musique? Dans son Journal, Klee nous surprend par son conformisme musical et son opinion dépréciative sur les musiciens de son temps : Bruckner, Mahler et Schoenberg. De même que ses recherches sur la figure du Carré Magique, vers 1923, gardent pour nous tout leur mystère... 


\title{
Innovation et conséquences
}

\author{
Histoire des problèmes compositionnels
}

\author{
Giselher Schubert
}

1 Theodor W. Adorno comprit dès 1928 que les problèmes compositionnels de chacune des œuvres d'Arnold Schoenberg étaient "rendus à ce point nécessaires par la façon de procéder dans l'œuvre précédente, qu'on pouvait penser qu'il s'agissait uniquement de résoudre les questions techniques qui y avaient été soulevées"1.

2 C'est au plus tard dès le début des années cinquante que cette réflexion d'Adorno s'est élargie à l'“Histoire des problèmes compositionnels" et s'est partout répandue; cette réflexion est à la base du développement de la technique compositionnelle de la nouvelle musique du $\mathrm{XX}^{e}$ siècle dans la mesure où celle-ci revendique son caractère obligatoire et voudrait se poser comme historiquement nécessaire. "Histoire des problèmes compositionnels" signifie qu'à l'instar du processus de résolution des problèmes dans l'histoire des sciences, l'acte compositionnel poursuit son développement selon une logique obligée: certaines dispositions compositionnelles, qui sont prises dans une œuvre et forment son caractère, voire sa teneur historique, se révèlent après coup, une fois l'œuvre achevée, incomplètes et imparfaites, et conduisent nécessairement à de nouvelles dispositions.

3 Ce processus ne peut, de par sa tendance, être achevé. Les compositeurs ressemblent à des chercheurs qui travaillent continuellement à résoudre des problèmes; leurs investigations et découvertes ont, dans cette continuité de la recherche, une place exactement définie objectivement et historiquement. Les œuvres sont comme les repères d'une évolution compositionnelle forcément progressiste, et la technique de composition, plus que les œuvres elles-mêmes, forme le théâtre où se prennent toutes les décisions de l'histoire de la musique. Un compositeur qui ne prend pas part à cette évolution, ou qui ne réussit pas à détecter, à reconnaître et à mettre à exécution ce qui est historiquement "nécessaire" et obligatoire, se met de ce point de vue à l'écart. Son œuvre apparaît "inutile", comme Boulez le formula une fois à propos de compositeurs qui se refusaient au sérialisme ${ }^{2}$.

4 L'acte compositionnel du XXe siècle, intégrant l'histoire de ses problèmes propres, a sa fondation dans l'œuvre de Schoenberg; il est repris avec vigueur par Boulez et 
Stockhausen et, passant par Ligeti et par nombre d'autres, il peut être repéré jusque dans la génération de compositeurs la plus récente. Bien sûr, son sens originel s'est presque déjà transformé en son contraire. Il regroupe par conséquent une part représentative de la composition avant-gardiste de ce siècle en une histoire de la musique véritable, essentielle, emphatique, derrière laquelle L'“histoire" d'autres développements, quand bien même ceux-ci sont perçus, ne fait plus que pâle figure.

Cette pensée compositionnelle progressiste, au sens de l'“histoire des problèmes compositionnels", est entrée, dès le début des années soixante-dix, en crise profonde avec la pensée scientifique du progrès. On ne vient plus guère raisonnablement à bout des conséquences toujours croissantes de ce que furent autrefois des innovations si fières et si souveraines. En outre, il est apparu, comme je le démontrerai plus loin, que l'utilisation, dans une situation déterminée, de ce qui fut un jour "usé", "dépassé" ou "inutile", peut se révéler comme la caractéristique d'un progrès compositionnel. Ainsi s'atténue sensiblement la possibilité de discerner sans malentendu entre le "progrès", dont on commence à se méfier, et la "restauration", à laquelle personne n'aspire plus guère sérieusement. Les conditions essentielles d'un jugement et d'une décision sûrs ne sont quasiment plus réunies, et de plus en plus, on sacrifie les différenciations et les différences sur l'autel d'un dogmatisme aussi aveugle que prétentieux, où l'attitude bornée passe pour un jugement sûr. Dans cette situation, on ne peut que dégager encore une fois, avec le plus d'impartialité possible, les lignes de développement de l'histoire de la musique, et défendre l'égalité de droits entre des approches divergentes.

\section{Technique dodécaphonique et logique harmonique}

6 Le point de départ d'une reconstruction de l'acte compositionnel intégrant sa propre problématique historique est constitué bien plus par le passage de la tonalité à la libre atonalité, puis par celui de la libre atonalité à la technique dodécaphonique, que par les développements à l'intérieur de la technique dodécaphonique de Schoenberg ou par les multiples passages de la technique dodécaphonique à la tonalité dans son œuvre tardive. La dissolution tonale, depuis le Deuxième Quatuor à cordes op. 10, va de pair avec le nivellement des structures thématiques et motiviques, voire de l'écriture musicale traditionnelle tout court; elle conduit aux procédés de la libre atonalité, lesquels incarnent le concept de "logique de l'expression", selon le terme d'Ernst Bloch - donc d'une "logique" de l'expression musicale : ils ont toujours une signification particulière, individuelle, et ne sont plus généralisables. Ce n'est qu'avec le perfectionnement de la technique dodécaphonique que Schoenberg va recourir à nouveau à des procédés technico-compositionnels généralisables et transmissibles; par ailleurs, la technique dodécaphonique va même reprendre les fonctions structurelles de la tonalité, sans pour autant lui servir d'ersatz : la référence au son fondamental de la tonalité est remplacée par une référence aux intervalles de la série fixés à l'avance. Parallèlement, Schoenberg renoue avec le travail thématique et motivique, et avec les formes traditionnelles fondées sur le thème et le motif. Si Schoenberg réussit de cette manière à allier l'unité individuelle et particulière des hauteurs engendrant la série dodécaphonique avec la logique aussi générale que traditionnelle de la pensée thématique, il est néanmoins une dimension de ses œuvres qui reste indéfinissable: l'harmonie, qui semble être due d'autant plus au hasard que les œuvres se rattachent ouvertement aux formes traditionnelles. Si l'on comprend par accords ou harmonies des phénomènes qui 
contrastent qualitativement de façon significative avec la somme des sons qui les composent - et le concept d'accord n'est rien d'autre-, alors on ne peut parler d'un ordre harmonique reconnaissable dans les œuvres dodécaphoniques de Schoenberg. Theodor W. Adorno s'enlise de manière frappante dans les problèmes que rencontre leur analyse harmonique. Il reconnaît dans "le principe de complémentarité" la "loi" qui en régit la progression harmonique ${ }^{3}$ : ce sont toujours les sons n'ayant pas encore été employés qui doivent servir à l'élaboration harmonique. Chaque progression complète le total des douze sons, qui apparaît comme un but et un accomplissement.

7 Cette idée d'Adorno entre en contradiction élémentaire avec un présupposé de la technique dodécaphonique, selon lequel les sons doivent se rapporter uniquement à eux-mêmes, et non pas à un quelconque phénomène supérieur. Aussi Adorno a-t-il laissé tomber cette théorie de "l'harmonie complémentaire", et il a ouvertement admis que l'harmonie, dans les œuvres dodécaphoniques, est le fruit du hasard. Cependant, il attire notre attention ${ }^{4}$ sur le fait que l'aspect aléatoire de l'harmonie pourrait être compensé par une logique tout aussi contraignante dans d'autres dimensions de 1'écriture, par exemple dans la conduite des voix. On ne peut nier que l'aspect aléatoire de l'harmonie peut être compensé par certaines dispositions compositionnelles; pourtant, il subsiste. Le problème de l'aspect aléatoire de l'harmonie, conséquence irrésolue de la technique dodécaphonique, est resté jusqu'à ce jour ouvert.

\section{Musique sérielle et musique aléatoire}

Curieusement, les compositeurs qui, à la fin des années quarante, ont trouvé dans la confrontation intensive avec la technique dodécaphonique leur propre position compositionnelle, n'ont pas envisagé le problème de l'aléatoire dans les œuvres dodécaphoniques, et ne l'ont pas résolu. Ces compositeurs, parmi lesquels Boulez fut nettement prépondérant, ne considéraient en aucun cas la série dodécaphonique comme un ensemble de rapports sonores, censés assumer la fonction de la tonalité, mais accentuaient, dans la technique dodécaphonique, le "principe sériel lui-même", ainsi que Boulez l'a nommés.

9 A partir de ce "principe sériel", ils se heurtaient à des problèmes de technique compositionnelle que, selon leur point de vue, Schoenberg n'avait pas encore perçus, mais que l'œuvre de Webern, elle, contenait en germe: la généralisation de la technique sérielle et son extension à toutes les dimensions de l'écriture musicale. Dans 1 'histoire des problèmes de leur activité compositionnelle, les passages de la tonalité à la libre atonalité, ou de la libre atonalité à la technique dodécaphonique, ou les problèmes internes de la technique dodécaphonique, qui auraient dû être "résolus", ne jouent aucun rôle. Les points d'ancrage de leurs recherches consistent bien plus à reconnaître et à imposer le "principe sériel lui-même", c'est-à-dire la musique sérielle.

La musique sérielle est née de la valorisation absolue d'une caractéristique précise de la technique dodécaphonique, celle du "principe sériel lui-même" (qui non seulement ne joue aucun rôle dans les œuvres dodécaphoniques de Schoenberg, mais que celui-ci a carrément tenté de résorber et de paralyser) : dès lors s'intensifient dans la musique sérielle les problèmes qui étaient non résolus dans les œuvres dodécaphoniques.

11 La technique dodécaphonique conduisait à l'aléatoire dans l'élaboration harmonique ; la musique sérielle, par contre, conduisait à l'aléatoire des événements harmoniques, 
mélodiques et rythmico-métriques; car la mélodie comme la rythmique sont, d'une manière à peine différente de l'harmonie, supérieures à la somme des éléments dont elles se composent, et elles s'en différencient qualitativement. Qu'elle le veuille ou non, la musique sérielle a toujours des moments mélodiques, rythmiques ou harmoniques, qu'elle ne réussit cependant plus à composer et à mettre en forme en tant que tels. C'est ainsi que la cohésion sonore effective des œuvres de musique sérielle a été recherchée du côté de principes toujours plus élémentaires, voire primitifs, tels que fort-doux, aigu-grave, son-bruit, lent-rapide, etc., comme cela s'est nettement dégagé du développement technico-compositionnel de Stockhausen dans les années cinquante. En toute logique, on accueillit, à la fin des années cinquante, à l'apogée de la composition sérielle, la musique aléatoire de John Cage, dont les procédés apparaissaient diamétralement opposés à ceux de la musique sérielle; cependant, comme le constatait György Ligeti en $1958^{6}$, musique sérielle et musique aléatoire ne se distinguaient plus entre elles par leurs résultats sonores: la musique totalement déterminée et la musique indéterminée se ressemblaient.

\section{Progrès et restauration}

Vers la fin des années cinquante, au début d'un renouveau compositionnel, György Ligeti n'a adopté ni les procédés de la musique sérielle, ni ceux de la musique aléatoire (qu'on ne pouvait plus guère développer); en revanche, il s'est associé au résultat de ces procédés : l'aléatoire, l'absence de forme ou le caractère anonyme de la mélodie, de la rythmique et de l'harmonie ont été poussés à tel point qu'ils n'étaient plus reconnaissables en tant que tels. Le renouvellement progressif des dimensions musicales traditionnelles, ou l'identification à celles-ci, apparaît aujourd'hui comme un progrès ou comme une échappatoire à ce nivellement complet de l'harmonie, de la rythmique ou de la mélodie. Ligeti traite ce phénomène avec une extraordinaire prudence et, à travers lui, saisit les inspirations les plus diverses : renouvellement de l'harmonie, de la rythmique, de la mélodie ou de la polyphonie, qu'il redécouvre tout simplement, avec d'autres compositeurs, comme des dimensions musicales propres à la composition. Le progrès compositionnel $\mathrm{du} \mathrm{XX}^{\mathrm{e}}$ siècle, pourrait-on dire de façon concise, revient ainsi à son propre point de départ, et il n'est pas encore bien établi si dans ce processus, la musique a atteint un niveau plus élevé, si elle s'est enrichie, ou si elle s'est appauvrie.

Ce développement, opéré par les compositeurs les plus divers, mais qui ressort dans l'œuvre de Ligeti comme particulièrement riche de conséquences sur le plan de l'histoire des problèmes, détermina la situation compositionnelle dans laquelle les jeunes compositeurs des années soixante-dix eurent à s'orienter. D'une part, cette situation résultait d'une histoire des problèmes compositionnels que l'on peut circonscrire d'une manière relativement exacte; d'autre part, elle se présente comme un fouillis inextricable des procédés les plus variés, qui n'ont plus de dénominateur commun; car il n'y a évidemment aucun avis unanime quant à savoir comment et jusqu'où il faut poursuivre ce renouvellement. L'histoire des problèmes compositionnels conduit ainsi à sa dissolution immédiate. Elle est aujourd'hui reconnaissable comme cas particulier, et ne peut plus longtemps masquer les riches perspectives de la musique du $\mathrm{XX}^{\mathrm{e}}$ siècle situées au-delà de l'histoire des problèmes. 


\section{Nouvelle objectivité}

14 Quand on parle de la musique de la "nouvelle objectivité" des années vingt, on pense avant tout à Weill ou Eisler en Allemagne, à Chostakovitch ou Mossolov en URSS, à Honegger ou Milhaud en France, parmi beaucoup d'autres compositeurs. Elle apparait maintenant comme une tendance compositionnelle qui rompt pour la première fois dans ce siècle avec le développement compositionnel immanent de la nouvelle musique, voire qui se laisse même consciemment dépasser par la composition "progressiste" déjà atteinte avec l'école de Schoenberg. "Autrefois, on se bagarrait pour des questions techniques, on s'insultait pour des détails, on construisait des visions de l'art et des visions du monde sur des sons et sur des relations entre les accords", trouve-t-on chez Hindemith aux alentours de $1929^{7}$, et l'on pourrait penser qu'il décrit là la situation des années cinquante; pourtant, il ajoutait, toujours en 1929: "Les questions techniques et purement artistiques passent quelque peu à l'arrière-plan. Ce qui nous concerne tous, c'est que l'ancien public disparaît: comment écrire, et que devons-nous écrire pour trouver un public plus large, différent et neuf? Où est ce public ?" En 1929, de telles réflexions ne visaient rien moins qu'à une transformation du concept de l'art. Il fallait faire éclater L"institution artistique", élargir le concept même d'art. On nivela les frontières rigides entre musique sérieuse et musique divertissante, on composa pour des buts précis, on écrivit de la musique pour les profanes, de la musique de film, de la musique pour la radio ; on écrivit de la musique qui devait être jouée, mais non plus forcément pour un public; on fit participer le public aux exécutions, on laissa intentionnellement les partitions inachevées, et on invita leurs utilisateurs à en compléter 1'écriture et à les terminer. En prenant ces mesures, on voulait, semble-t-il, résorber les conséquences qu'entraînait forcément le développement hermétique de la musique dans le cadre de 1'art institutionnalisé, qu'on n'arrivait toujours pas à dépasser au nom d'une quelconque vérité ou vraisemblance artistique, effectivement en jeu, mais dont personne ne pouvait dire ce qu'elle était. Pour les compositeurs de la "nouvelle objectivité", il s'agissait plutôt d'inscrire à nouveau la musique dans une pratique de la vie quotidienne, qu'elle semblait, de par son autonomie, avoir perdue complètement. Ils enlevèrent à la musique son aura, ils démystifièrent l'acte compositionnel. Cette attitude est encore particulièrement perceptible là où des problèmes spécifiques de technique compositionnelle sont thématisés, par exemple dans la théorie compositionnelle de Hindemith8 ${ }^{8}$.

Celui-ci s'efforce de faire correspondre les règles compositionnelles avec une "nature" universelle du son. Dans cette optique, tous les développements compositionnels et stylistiques de la musique, sur lesquels se base l'histoire des problèmes compositionnels, sont à considérer comme des aspects musicaux aléatoires, éphémères et caractéristiques d'une époque, qui n'ont aucune valeur contraignante, et dont on peut faire abstraction. S'il s'agissait, dans l'histoire des problèmes compositionnels, de reconnaitre ce qui doit être "historiquement nécessaire", il s'agit, dans la "nouvelle objectivité", de reconnaître ce qui est adéquat, utile, opportun; la "nouvelle objectivité" remplace par conséquent les critères du "progressiste", du nouveau ou de l'historiquement nécessaire, par ceux de l'utile, de l'opportun ou de l'adéquat. Cette différence s'explique aisément par les problèmes inhérents à la lecture des œuvres tonales tardives de Schoenberg. René Leibowitz, qui était convaincu du progrès nécessaire et absolu que représentaient l'atonalité et la technique dodécaphonique, 
propose une lecture "historico-philosophique" de ces œuvres: il distingue entre une histoire interne et une chronologie externe des œuvres de Schoenberg, rangeant les pièces tonales tardives avant les pièces dodécaphoniques; celles-là confirmaient après coup le chemin qui menait nécessairement à la technique dodécaphonique. Schoenberg aurait composé ces œuvres comme s'il avait été lui-même l'historien de sa propre évolution. Mais on peut aussi en dégager une autre lecture, à partir de la "nouvelle objectivité" : Schoenberg aurait composé ces œuvres de manière tonale parce qu'il les écrivait pour un usage déterminé, ou pour des types de distribution déterminés. Dès lors, la tonalité, l'atonalité ou la technique dodécaphonique constituent une valeur stylistique extérieure, la musique étant d'abord conçue au service d'un but.

\section{Populisme}

Dans les années trente et quarante, sous l'effet catastrophique de la crise économique mondiale, de l'exil des principaux compositeurs et de la Seconde Guerre mondiale, la "nouvelle objectivité" s'atténua pour devenir ce qu'on appelle le "populisme"; ce sont surtout les compositeurs américains qui usèrent de cette appellation. A cette période très amère, ils aspiraient à s'allier idéalement à leur public. Ils s'efforçaient de créer une musique de l'entente et de la solidarité, sans pour autant oublier le contexte musical historique de leur acte compositionnel. Marc Blitzstein, par exemple, qui fut l'un des rares compositeurs américains à étudier à la fin des années vingt auprès de Schoenberg à Berlin, distinguait en 1934 trois courants - les "primitifs" (par exemple : Bartók, Stravinsky, Varèse, Antheil), les "classiques" (Schoenberg, Hindemith) et les "populistes" (Weill, Milhaud) - et il observe ${ }^{9}$ que ces trois courants commencent à se confondre :

"Et que va-t-il en sortir? Si nous cherchons des exemples dans l'histoire, alors nous vivons la naissance d'une nouvelle grande forme". Blitzstein prédit en tous cas les grandes œuvres symphoniques qui apparurent dès le milieu des années trente dans tous les pays et dans tous les courants musicaux. Et en 1941 déjà, Aaron Copland ${ }^{10}$ reconnaissait dans l'œuvre symphonique de Chostakovitch le redémarrage le plus significatif dans le domaine musical depuis 1930: la tentative réussie d'écrire des œuvres symphoniques pour un nouveau public de masse. Il est vrai qu'avec la fin de la Seconde Guerre mondiale, avec la transformation du contexte social, ce développement s'interrompit rapidement. Il ne fut plus poursuivi que par des marginaux, dont on tient cependant aujourd'hui largement compte: Hartmann, Zimmermann, Killmayer ou Henze en Allemagne, Allan Petterson en Suède, Henri Dutilleux en France, pour ne nommer qu'eux.

\section{Absence d'orientation ou multiplicité}

$18 \mathrm{Si}$, sur cet arrière-fond, on se tourne à nouveau vers la composition contemporaine, alors les approches les plus diverses qui se sont développées de façon continue au cours de ce siècle, ou qui ont violemment contrasté entre elles, apparaissent immédiatement à l'esprit, et comme simultanément. Aucune approche compositionnelle ne semble être oubliée : nouvelle simplicité, néo-tonalité, musique post-sérielle, néo-expressionnisme, néo-dadaïsme, musique appliquée, post-modernité... les noms les plus variés donnés à la composition contemporaine recouvrent l'hétérogénéité, devenue incommensurable, 
d'une situation que l'on peut critiquer comme dépourvue d'orientation, comme flottante et chancelante, ou dont on peut vanter la multiplicité et l'ouverture. En même temps, ces noms accentuent également de façon polémique les positions, dans un combat artistique dont l'issue ne peut heureusement pas être prédite, mais à laquelle chacun aimerait donner durablement son empreinte. Personne n'attend que des approches divergentes coexistent paisiblement et se tolèrent entre elles; pourtant, personne ne devrait tenir ce contre quoi il se bat pour plus bête qu'il ne l'est peut-être lui-même.

\section{NOTES}

1. Adorno, Theodor W. : Schoenberg, Suite für Klavier, drei Bläser und drei Streicher op. 29, und Drittes Streichquartett, op 30 (1928), in Gesammelte Schriften, Band 18, Frankfurt 1984, p. 358.

2. Boulez, Pierre : Eventuellement, in Relevés d'apprenti, Paris, Seuil, 1966, p. 149.

3. Adorno, Theodor W. : Zweite Nachtmusik (1937), in Gesammelte Schriften, Band 18, a. a.0., p. 50.

4. Adorno, Theodor W. : Über das gegenwärtige Verhältnis von Philosophie und Musik (1953), in Gesammelte Schriften, Band 18, a. a.0., p. 168.

5. Boulez, Pierre : Moment de Jean-Sébastien Bach, in Relevés d'apprenti, op. cit. p. 17.

6. Ligeti, György : Wandlungen der musikalischen Form (1958), in die Reihe, Band 7, Wien 1960, p. 9.

7. Hindemith, Paul : Über Musikritik, in Melos 8, 1929, p. 106 sq.

8. Hindemith, Paul : Unterweisung im Tonsatz, I. Theoretischer Teil, Mainz.

9. Blitzstein, Marc : Towards a new form, in The Musical Quarterly 20 (1934), p. 213.

10. Copland, Aaron : Our new music, New York, 1941. 


\title{
La musique post-moderne
}

\author{
Produit de la société de consommation
}

\author{
Susumu Shono \\ Traduction : Viviana Aliberti
}

1 Contrairement à l'affirmation d'Adorno ${ }^{1}$, il faut reconnaître qu'aujourd'hui c'est l'auditeur-consommateur qui détermine la situation de la musique, que cela nous plaise ou non. En effet, à travers les débats sur la "nouvelle simplicité"2, il est apparu que le facteur "popularité" en musique ne pouvait être négligé. Partant, il convient de réaffirmer l'effondrement de l'avant-garde, ce qui signifie que nous sommes maintenant dans la période post-moderne de la musique. La génération des compositeurs venus après cage et les musiciens post-sériels peuvent se référer en toute liberté à n'importe quels matériaux et formes historiques. On peut apercevoir dans leur musique ("minimalisme", "post-minimalisme", ou "nouveau romantisme") une tendance générale à 1'éclectisme, ainsi qu'un net changement dans la conscience historique. Ces mêmes caractéristiques se retrouvent également dans la musique moderne - néo-classicisme - ainsi que dans la musique populaire en général. Comment l'expliquer?

\section{L'éclectisme comme poétique de la musique post- moderne}

On pourrait citer le Grand Pianola Music (1982) de John Adams comme un exemple typique de musique post-moderne. Dans la structure de cette œuvre répétitive, on reconnaît aussi bien les sons d'une fanfare avec tambours, le style pianistique des concertos pour piano de Beethoven, le type d'instrumentation propre à Wagner, et ainsi de suite. Comme les compositeurs peuvent tout mettre dans une telle structure répétitive, on aboutit à la juxtaposition de matériaux sonores hétérogènes. On trouve souvent les caractéristiques de ce genre d'éclectisme dans la musique contemporaine : un style expressionniste dans le "nouveau romantisme" ; un matériau tonal ou modal et des éléments non-européens dans la musique minimale ; beaucoup de styles musicaux historiques différents, des éléments empruntés à des genres divers, etc. 
3 Apparemment, il semble exister une parenté entre ces tendances et celles de la pratique citationnelle en musique. Mais il n'existe pas nécessairement d'intention sémantique dans l'éclectisme mentionné ci-dessus. Les éléments historiques et noneuropéens n'ont pas toujours pour fonction de représenter le passé, tel pays ou telle région. Dans bien des cas, chacun d'eux n'est qu'un matériau ou un élément parmi d'autres. Par ailleurs, il y a une différence décisive entre les tendances éclectiques et celles fondées sur la citation. Dans le premier cas, il n'existe ni développement traditionnel d'un motif en phrases, ni organisation; l'unité musicale manque par conséquent souvent. Mais ce manque est perçu par les tenants de l'éclectisme comme un plus, alors que pour ceux qui pratiquent la citation, il est souvent l'indice d'un défaut.

4 En effet, dans la conception traditionnelle, c'est le compositeur qui doit unifier le matériau en une œuvre d'art: le manque d'unité apparaît comme le signe de son incapacité. Cependant, dans le cas de l'éclectisme contemporain, ce n'est pas le compositeur mais l'auditeur qui donne au matériau son unité; il crée ainsi sa propre version.

5 Cette différence se révélant importante pour comprendre ce qu'est la musique postmoderne, il nous faut l'étudier plus en détail. L'un des problèmes les plus difficiles auxquels les compositeurs du vingtième siècle ont été confrontés est, comme l'on relevé Paolo Emilio Carapezza et Joachim M. Benitez ${ }^{3}$, la "déconstruction" du matériau du point de vue de la structure. D'un côté, les compositeurs européens d'avant-garde ont abordé ce phénomène de manière négative. Pour Karlheinz Stockhausen, “l'ordre sonore signifie donc une subordination des divers sons à un principe unitaire représenté et une absence de contradiction entre l'ordre au niveau particulier et l'ordre au niveau général" 4 . Il s'est donc appliqué à résoudre la contradiction par un nouveau principe : le principe sériel. Et Pierre Boulez a aussi insisté sur la nécessité d'élargir et d'appliquer ce principe à tous les aspects de la musique. Quoi qu'il en soit, tous perçoivent cette "déconstruction" comme négative et comme devant faire par conséquent l'objet d'une amélioration. Mais d'un autre côté, parallèlement à la lignée Schoenberg-Webern-Boulez et Stockhausen, on peut en trouver une autre. Pour être plus précis, il ne s'agit pas d'une lignée qui se développe, mais d'un ensemble de compositeurs isolés, ayant parfois perdu tout lien entre eux, et percevant cette "déconstruction" de manière positive. Pour eux, cela signifie qu'il est désormais possible d'utiliser n'importe quel matériau sonore, y compris ce qui n'avait jamais été utilisé comme matériau musical, et ce, le plus librement du monde. On peut mentionner ici les noms de Satie, Busoni, Sorabji, Stravinsky, Ives et même, en faisant retour jusqu'au dix-neuvième siècle, celui d'Alkan (Morhange) comme des exemples précoces de cette tendance, aussi bien que les compositeurs post-modernes contemporains: les représentants du minimalisme, du Nouveau Romantisme, de l'expérimentalisme anglais et autres.

\section{“Pseudomorphose" sur la logique de l'image}

6 Prenons pour exemple typique la déconstruction dans la musique de Satie. La musique d'Entr'acte, composée par Satie pour Relâche (1924), consiste en de brefs motifs répétés, qui correspondent chacun à une image tournée par René Clair. De même que les images n'ont aucune cohésion, la musique, qui recourt à des procédés de transformation et de 
développement du matériau ou à des relations contrastées, manque d'unité. Comme toute la structure est déterminée par l'image - dans un rapport d'antériorité ou de simultanéité, en tout cas d'indépendance -, la méthode de composition consiste à disposer des matériaux sur un fond de continuité qui dépend entièrement de la structure de l'image. Mais Satie lui-même prend cette construction, très lâche du point de vue de la tradition, de manière positive. En réalité, il se sert de la même méthode dans son Socrates ou dans Parade. La première de ces pièces est composée dans l'esprit de la Musique d'ameublement ; aussi, les relations entre les sons et leur atmosphère $y$ sont-elles plus importantes que l'unité sonore. Dans la seconde, on trouve des matériaux tel que le bruit d'une sirène, d'un pistolet ou d'une machine à écrire, matériaux dont on peut difficilement se servir musicalement dans le cadre d'une structure symétrique et géométrique totalement indépendante des caractéristiques des matériaux sonores. On sait que Cage, influencé par cette idée, avait développé dans les années quarante une structure rythmique en "racine carrée". Aujourd'hui, on peut faire de la musique qui consiste en du bruit et du "silence".

7 Soit dit en passant, la méthode de composition d'Entr'acte est très semblable au style des musiques qui accompagnaient les films muets. Au début de ce siècle, chaque maison de production avait l'habitude d'envoyer aux salles de projection des notices musicales, à savoir "des programmes imprimés de musique instrumentale propre à accompagner leurs films" . Et de "larges échantillons de musique de scène, minutés et classifiés selon leur caractère et tempo"6, étaient plaqués sur le continuum de chaque film. Le résultat de cette méthode a nécessairement un caractère éclectique, cela manque d'unité musicale. Ainsi, il devient aussi clair que, du point de vue de l'unité musicale et de la méthode compositionnelle, il n'existe pas de différence entre la musique de Satie et la musique d'accompagnement de films, laquelle représente la culture populaire dans la société de consommation.

On peut parfois trouver les mêmes tendances dans la musique néo-classique de Stravinsky. Il y a, par exemple, dans Pulcinella, des matériaux empruntés à la musique du dix-huitième siècle aussi bien qu'à l'orchestration moderne. Et la structure globale de cette œuvre est déterminée par celle du ballet. En ce sens, Stravinsky traite les matériaux en artisan, suivant une logique qui est celle de l'image, celle d'un cadre structurel pré-existant (et non le sien propre) sur lequel il vient disposer des matériaux dont il raffine les sonorités. Bien que parfois, le point de départ de sa musique ne soit pas une image mais la projection d'une forme établie de la musique instrumentale, il use de celle-ci tout comme du cadre structurel objectif dans lequel il peut disposer les matériaux librement.

Remontons maintenant notre filière jusqu'à un singulier compositeur français du dixneuvième siècle : Alkan (Morhange) ${ }^{7}$. Une des caractéristiques les plus intéressantes de sa musique est la coexistance de la forme classique avec la sonorité romantique. Selon les termes de Ronald Smith, "la musique d'Alkan est romantique, dans la tradition de Berlioz - pleine d'imagination et de couleurs, poignante, dégageant un lyrisme sobre et sans volupté, mais profondément émouvant - et cela à l'intérieur d'un cadre fermement enraciné dans le style classique". En effet, dans sa Sonatine Op. 61 par exemple, on peut reconnaître, dans le premier mouvement, le schéma rigide de la forme sonate basé sur une structure périodique de huit mesures. Cependant, dans le cadre de cette structure, des matériaux hétérogènes sont juxtaposés, tels un passage pianistique de Chopin, une mélodie romantique de Berlioz, un hymne archaïque, une texture proche du cluster, et 
ainsi de suite. Il en résulte pour l'oreille des fragments qui changent brusquement et qui ont chacun leurs propres sonorités particulières; pour cette raison, la musique d'Alkan fait l'objet de critiques aussi négatives que celles que l'on peut relever dans la description de Georges Beck: "il faut dès l'abord, dit-il, signaler deux défauts: la prolixité et l'inégalité"'. Quand bien même les œuvres d'Alkan auraient de tels défauts, la préoccupation du compositeur n'est pas d'établir d'étroites relations entre le tout et les parties, mais d'affiner chaque détail avec le même souci qu'un maître artisan. On peut ajouter Kaikhosru Shapurji Sorabji à cette famille de compositeurs. Dans sa musique extravagante, comme son Opus clavicembalisticum, on peut voir la juxtaposition de passages hétérogènes, exagérément décoratifs, merveilleusement raffinés et hautement sophistiqués.

10 Dans le domaine de la musique populaire produite aujourd'hui, les musiciens utilisent les mêmes méthodes que dans la musique éclectique. Ils disposent des matériaux variés (textures et sonorités empruntées à des musiques non-occidentales, sons électroniques ou synthétisés, bruits urbains de le vie de tous les jours, sons naturels, etc.) sur le fond solide d'une structure rythmique faite par des percussions ou des machines à rythmes. Il n'est pas difficile d'entendre partout de tels rythmes, surgissant par exemple d'un "walkman" voisin... On retrouve les mêmes caractéristiques dans les vidéo-clips ${ }^{10}$. Il me semble que les sons de telles musiques perdent tout sens parce qu'ils ne sont pas soutenus par des structures immanentes nécessaires pour les relier entre eux; et c'est pourquoi les musiciens recourent aux structures hétérogènes de schémas rythmiques ou d'images. On peut étudier, dans ce contexte, l'opéra contemporain, un des genres les plus actifs de la musique post-moderne. Les éléments de musique populaire, ainsi que la simplicité structurelle de chaque partie, sont pensés en vue d'être acceptés par la société de consommation; de même, les biographies bien connues d'Einstein, Ghandi ou Nixon sont là pour donner à l'œuvre, de l'extérieur, un semblant d'unité. Dans ce sens, l'opéra contemporain se rapproche de la musique de films, de l'illustration sonore à la télévision ou dans la vidéo. Comme l'a écrit Adorno: “...toutes les œuvres d'art musicales unies se placent sous le signe de la pseudomorphose sur le langage verbal" ${ }^{11}$. Ainsi, en ce qui concerne la musique post-moderne représentée par l'opéra contemporain, nous devons nous référer à une "pseudomorphose" sur la logique de l'image au cinéma ou en vidéo. C'est ainsi que se comporte la musique post-moderne dans la société de consommation.

\section{La musique du passé comme milieu et environnement}

Hugh Macdonald a relevé qu'Alkan composait sa musique en "style ancien"12, et Daniel Charles le cite comme le précurseur des compositeurs post-modernes, lesquels ne recherchent pas la nouveauté en musique ${ }^{13}$. Ainsi, contrairement au "je suis né trop tôt" des avant-gardes, ces compositeurs semblent dire: "je suis né trop tard". Ceci montre chez eux une conscience historique différente de celle des compositeurs d'avant-garde.

12 Avant d'étudier les caractéristiques de la conscience historique des compositeurs postmodernes, il nous faut saisir celle qui est propre aux modernes. Beethoven est, parmi les compositeurs, l'un de ceux chez qui cette conscience historique moderne est clairement manifeste. Comme l'a fait remarquer Martin Zenck $^{14}$, dans les Variations Diabelli, Beethoven, en récapitulant tous les styles passés de la variation, y compris son 
propre style dans sa période intermédiaire, établit un nouveau style, complètement différent de celui du passé. Dans cette attitude, on peut reconnaître deux caractéristiques de la conscience historique moderne : continuité et différence. D'un côté, en résumant leurs styles de la $31^{\mathrm{e}}$ à la $33^{\mathrm{e}}$ variation, Beethoven définit sa position par appartenance au groupe Bach, Haendel, Haydn et Mozart; il importe qu'il suive la ligne orthodoxe et continue de la musique occidentale. Mais d'un autre côté, il sent qu'il doit marquer la différence entre son style propre et les styles du passé. Et c'est cette différence même qui fait la valeur de son propre style. Ce processus de différenciation fait l'histoire de la musique occidentale orthodoxe, jusqu'à notre siècle et ses avant-gardes. Schoenberg n'a-t-il pas lui-même accentué sa filiation avec Beethoven, en passant par Brahms? A ce point, nous pouvons caractériser l'institution de "l'esthétique de la nouveauté" comme une attitude essentielle des compositeurs modernes, sans oublier cependant qu'elle ne dépend que d'une logique propre à la production musicale. Et tant que durera la société industrielle - dans laquelle la logique de la production est en général dominante - nous pourrons dire avec Adorno que c'est la manière de composer qui détermine la situation présente de la musique.

13 La conscience historique de celui qui "reproduit" la musique est assez différente. Bien que l'interprète reproduise toujours des œuvres du passé, et qu'en ce sens, il ait toujours à faire à lui, ce dont il a conscience, curieusement, c'est de la disparition de toute distance entre passé et présent. En effet, bien qu'il doive interpréter des œuvres du passé, il le fait au présent, et bien que tout ce qu'il interprète appartienne au passé, il est moins conscient de la différence entre passé et présent que de la différence entre les œuvres. Un interprète choisit, en vue d'un "répertoire", un ensemble d'œuvres qui, en tant qu'objets, ont des droits égaux, qui méritent toutes d'être jouées et acquièrent de la valeur seulement par comparaison entre elles. En ce sens, l'œuvre musicale fait partie des alternatives historiquement neutres. C'est comme si l'on retirait toute historicité à chaque œuvre musicale. Dans ce contexte, l'attitude de l'interprète est semblable à celle du consommateur. Interpréter une pièce revient à la choisir parmi toute une série d'objets possibles, suivant le désir de la consommation. Interprétation comme consommation: les interprètes constituent leur "répertoire" présent en supprimant toute historicité aux œuvres du passé. (Bien sûr, l'interprétation en tant que reproduction comporte aussi un aspect propre à la production. En effet, si un interprète est conscient d'avoir un style bien à lui, qui se démarque de celui d'un autre, on aura là, au moins en matière de style, l'avant-garde de l'interprétation. Car la conscience historique d'un tel interprète est identique à celle des compositeurs modernes.)

14 A ce point, il convient de se rappeler que beaucoup de compositeurs "hérétiques" comme Alkan, Busoni ou Sorabji, sont des compositeurs-interprètes. Quand ils composent, ils traitent les œuvres musicales du passé en dehors de toute considération historique, sur la base de leur expérience en tant qu'interprètes. D'où leur éclectisme. On pourrait dire qu'ils traitent l'histoire (c'est-à-dire la musique du passé) comme leur milieu et leur environnement, tandis que les compositeurs modernes lui conservent son historicité ${ }^{15}$. Quant aux compositeurs post-modernes contemporains, ils ont la même conscience historique que ceux-là, non pas qu'ils soient eux aussi des compositeursinterprètes, mais parce qu'ils sont pris aujourd'hui dans la société de consommation. 


\section{De la notion d'"expression" à celle d"'être témoin"}

15 A ces compositeurs post-modernes qui traitent l'historique comme un contexte, on peut ajouter le nom de Ferruccio Busoni. D'une part, en tant qu'interprète, il jouait le répertoire de Bach à ses contemporains, y compris Alkan. D'autre part, en tant que compositeur éclectique, il pouvait utiliser des éléments stylistiques du baroque jusqu'à l'expressionnisme ou au néo-classicisme. Bien qu'il anticipât l'organisation de microintervalles, il transcrivit également de nombreuses pièces pour piano de Bach. Ce sont ces transcriptions qui révèlent un type de conscience historique tel qu'il permet de traiter la musique du passé comme milieu et environnement.

Mais il est encore plus important de voir comment nous évaluons les transcriptions de Busoni par rapport au reste de ses compositions. On s'aperçoit, en lisant la liste de ses œuvres dans n'importe quel dictionnaire de musique, que les transcriptions arrivent bonnes dernières et comme en ajout. En raison de leur manque d'originalité, on ne leur a accordé qu'une importance relative. Seules les œuvres originales ont une véritable valeur. Mais cette manière d'établir une différence - propre à la modernité et à la manière qu'elle a d'envisager l'œuvre musicale - perd beaucoup de son sens en regard des activités compositionnelles de Busoni. En effet, on peut aussi trouver dans sa production une continuité - qui va de l'édition des œuvres de Bach (Busoni-Ausgabe), en passant par l'arrangement, la transcription et la recomposition de ces œuvres (BachBusoni gesammelte Ausgabe), la composition (fantaisie) sur la base de thèmes de Bach (ou d'autres) et le façonnement d'un style, jusqu'aux compositions "originales". Pour Busoni, il n'y a pas une différence de valeur entre tout cela. "Chaque notation, dit-il, est déjà la transcription d'une certaine idée abstraite... Une idée devient sonate ou concerto, et ces derniers sont eux-mêmes déjà un arrangement de l'original." ${ }^{16}$ De ce point de vue, tout est transcription ou arrangement. Pour Busoni, la musique est le plus souvent transcription. Il tient cela de Bach. En effet, Bach n'est-il pas le plus grand des arrangeurs? De plus, Busoni a dit lui-même : "L'interprétation d'une pièce de musique est une transcription. ${ }^{17}$ On voit se dégager ici une ligne indifféremment continue entre la transcription, la composition et l'interprétation. Chaque activité musicale est transcription. Et à cette chaîne, on peut légitimement ajouter l'écoute.

17 La valeur d'“originalité" qui étayait solidement les structures esthétiques centrées autour des œuvres musicales de l'âge moderne perd son sens dans la musique postmoderne. En particulier, on ne peut trouver dans la conception busonienne de la transcription l'idée selon laquelle un génie musical individualisé poserait quelque chose comme origine unique d'une œuvre musicale. Nous nous trouvons maintenant dans la sphère de la "théorie du texte". Ce que nous pouvons entendre des œuvres de Busoni n'est pas produit par lui seul, mais par les styles des compositeurs contemporains, ceux de l'époque de Bach, parfois ceux des prédécesseurs de Bach, et ainsi de suite. D'où l'éclectisme.

Mais il est plus important encore de relever que, par delà le changement de la conscience historique moderne et la dépréciation du concept d'“originalité", c'est l'idée même d'expression qui perd son fondement. Car c'est la conscience historique moderne et l'esthétique de la nouveauté qui suscitent la dialectique de l'expression. Pour exprimer quelque chose de nouveau qui n'a pas encore été exprimé, il est nécessaire de créer un moyen parfaitement inédit, différent de tous les autres. Or les compositeurs postmodernes considèrent qu'ils peuvent utiliser n'importe quel style comme 
environnement dans leurs compositions, si bien que les moyens d'expression sont de toute manière indifférents. Leur travail consiste à juxtaposer ou à produire des matériaux musicaux pour les auditeurs-consommateurs, qui apprécient quant à eux le jeu des différences de sonorités dans une pièce. On ne peut donc trouver là aucun sujet de l'expression musicale, et pas davantage de contenu. Ce que l'on peut écouter, c'est le jeu des différences, à savoir le texte ou la surface de la sonorité. En second lieu, l'idée d'"expression" perd son fondement dans la mesure où le sujet de l'expression, la personne du compositeur moderne, perd son droit de propriété unique des œuvres musicales. Comme nous l'avons vu dans le cas des transcriptions de Busoni, il n'est pas possible de décider quel est le sujet de "ses œuvres". Quant à l'auditeur-consommateur, un producteur ou groupe de producteurs lui offrent différentes sortes d'“événements" musicaux; il importe que le producteur les donne au consommateur sous la forme d'une juxtaposition, afin que le consommateur jouisse de leurs sonorités indépendemment les unes des autres. Dans ces conditions, il est inutile d'assigner le contenu de ces sonorités fragmentaires au sujet de l'expression. L'idée moderne d'“expression" est dévaluée.

19 Ce n'est plus l'idée d'“expression" qu'il faut discuter, mais celle d'"être témoin"18. Avec la musique post-moderne, l'expérience du consommateur se résume à être présent là où ont lieu les événements musicaux. Le consommateur-auditeur est le témoin d'un certain événement musical - détaché de ses relations musicales avec d'autres événements ou avec la totalité de l'œuvre musicale - et ce, dans le cadre des situations arbitraires qu'il établit. En effet, comme les sonorités elles-mêmes manquent de sens, l'auditeur-consommateur produit des sens musicaux en insérant celles-ci dans des situations arbitraires qui ne leur sont pas spécifiques. On comprend mieux, dans ce contexte, l'émergence des "performances", ou le récent regain de l'opéra. On peut aussi mentionner les présentations dramatiques au moyen d'éléments visuels dans le domaine de la musique populaire, laquelle revêt les caractéristiques de la musique propre à la société de consommation.

La post-modernité en musique est donc une caractéristique - qu'on peut aussi trouver dans la production des compositeurs hérétiques de la première moitié du siècle - en rapport avec la consommation musicale. L'âge post-moderne de la musique a véritablement commencé après Cage, alors qu'on assistait à la fin de la dialectique de 1'expression et à l'essor de la société de consommation. Là, le style éclectique domine et l'auditeur-consommateur est témoin d'événements musicaux qu'il insère dans une situation arbitraire. Nous devons toutefois réaffirmer que la post-modernité n'est pas une caractéristique à rechercher, mais une situation de fait qu'on ne peut négliger et qu'il faut accepter. Du moins pouvons-nous faire l'étude de la musique post-moderne, et la mettre en relation avec la structure de la société de consommation.

\section{NOTES}

1. Adorno, Theodor W. : Philosophie der Neuen Musik, Gesammelte Schriften, Bd. 12, Frankfurt, p. 9. Traduction française, Paris, Gallimard 1962. 
2. Cf. Kolleritsch, Otto, éd. : Zur "Neuen Einfachheit" in der Musik, Wien, 1981.

3. Cf. Carapezza, Paolo Emilio : De Modi Musicandi, Collage no 9, 1970, et Joachim M. Benitez, Gendai Ongaku o Yomu - Ecriture o koete, Tokyo, 1981, p. 24.

4. Stockhausen, Karlheinz: Texte zur elektronischen und instrumentalen Musik, Köln, Dumont Schauberg, 1903, Bd. 1, p. 18. Traduction française in Contrechamps, 9, 1988, p. 11.

5. G. B. Anderson, The Presentation of Silent Films, or Music as Anaesthesia, in The journal of Musicology, vol. V, no. 2, Printemps 1987, p. 283....

6. Anderson, Gillian B. : The Presentation of Silent Films, or Music as Anaesthesia, in The journal of Musicology, vol. V, no. 2, Printemps 1987, p. 283.

7. J'ai discuté plus en détail de sa musique dans Shono, Susumu: Alkan, Gendai siso (Revue de la pensée d'aujourd'hui), vol. 11 - 1, 1988, pp. 214-221. C'est la première des séries “Onkagu no Fukei" qui traite de la musique post-moderne.

8. Smith, Ronald : Alkan : the Enigma, London, 1976, p. 104.

9. Beck, Georges éd., Alkan, Charles Valentin: Oeuvres choisies pour piano, Le pupitre, XVI, Paris, 1969.

10. Dans ce genre, les sons n'acquièrent leur sens que par l'intermédiaire de la construction de l'image, des parcelles de chaque image et d'autres éléments visuels.

11. Adorno, T. W : op. cit., p. 121. Traduction française par Hans Hildenbrand et Alex Lindenberg, Paris, Gallimard, 1962, p. 136.

12. Macdonald, Hugh : Alkan, in The New Grove Dictionary of Music and Musicians, vol. 1.

13. Cf. Charles, Daniel : Le son comme image du temps, in Revue d'esthétique, 7, 1984, pp. 103-108.

14. Cf. Zenck, Martin: Rezeption von Geschichte in Beethovens Diabellivariationen - Zur Vermittlung analytischer, ästhetischer und historischer Kategorien, in Archiv für Musikwissenschaft, Heft 1, 1980.

15. Ceci s'appuie sur la conférence qu'a tenue Joachim M. Benitez au congrès de la société musicologique du Japon en 1987.

16. Busoni, Ferruccio : Wesen und Einheit der Musik, éd. Max Hesse, 1956, p. 125.

17. Busoni, Ferruccio : Wesen und Einheit der Musik, éd. Max Hesse, 1956, p. 125.

18. Cf. Armstrong, Robert Plant: The affecting presence: an essay in humanistic anthropology, Urbana, 1971. 


\title{
Musique et référence
}

\author{
Christian Corre
}

$1 \quad L a$ question de la Référence en Musique n'est plus d'une brûlante actualité : non seulement parce que les compositeurs ont su, depuis vingt ans, nous familiariser avec les pratiques s'y rapportant (citation, collage, transcription, réécritures diverses), mais aussi parce que le statut de ces pratiques a évolué "dans les esprits", sur le plan moral comme sur le plan intellectuel. Nulle polémique à espérer sur ce terrain, si effectivement leur reconnaissance, leur légitimation, voire leurs lettres de noblesse sont aujourd'hui des évidences. Pourquoi dès lors parler d'une "question"? Peut-être parce qu'à cette question, il y a déjà trop de réponses : "art au second degré", "règne du simulacre", "empire du faux", "configuration finale" 1 , tout un champ sémantique a eu le temps-entretemps-, de se déployer, c'est-à-dire de s'interposer. Et toutes ces idées neuves n'ont pas échappé à leur destin: devenir à leur tour des idées reçues. La méfiance d'un Dahlhaus envers l'expression de "post-moderne" ("un slogan très suspect" ${ }^{\prime 2}$ est bien motivée par ce qui, en elle, relève du slogan. C'est dire que la question reste bien en souffrance d'une interprétation, "si tout n'est pas duperie", comme s'exclamait jadis Adorno!

2 Mais dénoncer les lieux communs ne suffit pas : car il est également vrai que la prise de recul nécessaire à la compréhension de telles musiques ne peut plus vraiment être ce comportement élémentaire, spontané, dont nous avions l'habitude vis-à-vis de n'importe quelle actualité. "Prendre du recul", c'est aussi être habité par un désir: celui de trouver la bonne distance à partir de laquelle pourra se constituer une séquence temporelle cohérente, un ensemble significatif ; c'est déjà faire de l'Histoire. Or il semble que le penchant naturel des formes d'art ici envisagées soit précisément de se dérober à une telle visée, de ruiner toute tentative d'insertion dans un discours "historien" - pour autant que l'histoire n'y est plus prolongée sous forme d'avancée supplémentaire, mais bien plutôt directement convoquée à travers ses produits amputés de leurs tenants et aboutissants. Transhistoriques par nature, elles ne sauraient se constituer elles-mêmes comme moment historique, elles ne seraient pas viables épistémologiquement... ${ }^{3}$

3 Ne peut-on pas, vaille que vaille, tenter au moins une généalogie, quitte à remonter assez loin? Le fait qu'on ose à peine donner en exemple la Sinfonia de Berio, et autour 
d'elle Hymnen, Ludwig Van, Ombres, et quelques autres pièces datées des années soixantedix, est déjà un indice : ces œuvres sont si connues, si commentées, leur regroupement chronologique tellement serré qu'il est impossible de ne pas les considérer comme des phares. Elles sont emblématiques, inaugurales... Mais en quoi ? La capacité d'une œuvre à devenir ainsi un "classique" dépend beaucoup (indépendamment de sa réussite esthétique), de sa capacité à se laisser traverser, ou porter, par des discours : lorsque Berio évoque la "Traumdeutung" d'une part, l'analyse de la musique par elle-même d'autre part ${ }^{4}$, il désigne bien ce qui, dans sa production, recèle une richesse symbolique réelle (l'extraordinaire réseau d'implications qui fait d'une œuvre une grande œuvre) mais il bénéficie aussi d'une certaine disponibilité de ces notions au moment opportun (celui du succès de la relecture de Freud par Lacan, celui où Lévi-Strauss ne craint pas de voir en Wagner le père de l'analyse structurale). La validité théorique de celles-ci dans le champ musical (incidence du travail du rêve sur la composition, statut exact d'une analyse ne recourant pas à un détour verbal) n'est nullement assurée, mais au fond n'a pas à l'être : il suffit que ces maîtres-mots - ces "signifiants" si l'on veut soient dynamiques, stimulants, tant pour la création que pour la recherche. Des concepts comme "objet sonore", ou "espace musical", émoussés aujourd'hui, mais très opératoires hier, projettent également sur la citation leur éclairage instantané, et peuvent encore aider à la définir. Enfin, il en va de même pour tout l'aspect "politique" du problème: emprunts ou réappropriations esthétiques veulent aussi dire abolition des droits d'auteurs et de la propriété intellectuelle. ${ }^{5}$

On peut trouver confirmation de tout cela dans le destin bien différent d'un Ives ou d'un Zimmermann : des compositeurs chez qui ces techniques ont pris la plus grande extension, au point de devenir une composante essentielle de leur style, mais qui n'ont pas eu, en tant que prédécesseurs, la même "chance" culturelle, connaîtront un long purgatoire. En revanche, la recherche musicale à vocation scientifique de ces mêmes années, dans sa prétention à recueillir l'héritage de la linguistique, (il s'agissait de rien moins que d'une "fondation"...) semble avoir eu bien trop à faire avec les objets musicaux de premier degré pour s'intéresser à tout ce qui pouvait relever du second... ${ }^{6}$ A cet égard, l'attitude des compositeurs eux-mêmes n'était pas dénuée d'ambiguïté : Berio se défendra d'avoir "voulu faire un collage", Kagel d'avoir "parodié" Beethoven, comme si ces mots sentaient encore le soufre, et devaient s'épuiser dans leurs connotations régressives ou ancillaires - attitude peu différente au fond de celle des zélateurs du Stravinsky d'avant-guerre, acharnés à laver leur idole de tout soupçon (plagiat, pastiche, exercice de style, etc.). D'ailleurs, la stratégie citationnelle restera la plupart du temps, du moins sous des dehors aussi spectaculaires, une exception, un point-limite dans l'itinéraire de chacun de ces musiciens, une "expérience" qui ne se distingue du concept général de "musique expérimentale" que par des répercussions éthiques peut-être plus embarrassantes. Ce qui n'en limite nullement la portée, au contraire: il faut se demander ce que cette expérience, confrontée aux autres, aura conquis ou remis en cause dans son domaine propre.

Une première remarque s'impose: citation et collage ne sont pas seulement des conduites musicales particulières, au sens où peuvent l'être d'autres procédures ("ouverture", "aléatoire", "improvisation", "électro-acoustique", qui furent et restent des notions indépendantes en dépit de toutes leurs interférences dans la pratique). Elles occupent aussi une place définie dans le contexte de la seule "référence" : ce sont elles - et non pas, par exemple, les translitérations et transpositions de type stravinskyen - qui dominent à ce moment-là. Pour reprendre la classification de Gérard 
Genette, elles sont intertextuelles, plus qu'hypertextuelles... Ce qui signifie que le travail de réécriture opéré sur le ou les "originaux" peut être pratiquement nul, et que, s'il y a transformation, celle-ci est seconde par rapport aux tactiques de montage ou d'assemblage. Le fameux "geste" citationnel, généralisé dans le collage, est de loin le plus brutal, le moins cultivé de tous ceux qui, en musique, relèvent de l'imitation. D'un coup, l'écart qui le sépare du sens de la composition alors en vigueur, tournée pour l'essentiel vers une expansion de la pensée sérielle, n'en apparaît que plus surprenant, d'autant que ce sont les mêmes compositeurs qui passent de l'un à l'autre. L'antinomie semble irréductible, entre une passion de la syntaxe, en quête des conditions et des moyens grâce auxquels de nouvelles œuvres seront possibles, et une frénésie de l'emprunt, exercée sur des objets préfabriqués réinsérés tels quels ou presque, de toute façon reconnaissables, et tirant même du fait de pouvoir l'être leur véritable fonction musicale ${ }^{7}$. L"assemblage", la "juxtaposition", dans leur principe, tournent d'abord le dos à ce que la composition demande (synthèse, intégration), et en l'absence de système supérieur, descriptible extérieurement à l'œuvre, tendent à l'énumération, à l'accumulation érudite, et au-delà au pur "processus"...

6 Une telle opposition doit être elle-même interrogée, puisqu'aussi bien c'est elle que le collage met en mouvement, puisque c'est en elle que s'enracinent nombre de réticences et de malentendus. Certes, un travail comme la $10^{e}$ Symphonie de Pierre Henry ne choque plus personne; pourtant, l'idée d'un matériau homogène, fût-il propre à une seule œuvre, la recherche d'une cohérence interne, fût-ce à partir de multiples readymades, représentent encore de sérieuses garanties ${ }^{8} . .$. Et sans doute faut-il ici séparer "citation" et "collage", grâce à une tierce notion qui les traverse l'un et l'autre, et permet de les articuler différemment; celle d'hétérogénéité. On peut envisager le collage comme superposition de "couches" stylistiquement ou grammaticalement étrangères les unes aux autres, sans répétition stricte d'un énoncé antérieur (ce que suppose la citation pure). Tels, par exemple, La Question sans réponse de Ives ou exemple plus récent - le début de telle pièce de Heinz Holliger (Turm Musik). En revanche, la citation tire sa puissance disruptive - son hétérogénéité propre - de son statut d'énoncé répété : dans ce second cas, le collage (le mini-collage s'il s'agit d'une citation unique) est commandé par la répétition, il en est l'effet direct dans l'écriture. Dans le premier cas, le collage est la perspective générale du travail, le point de vue à partir duquel on va recueillir puis associer des objets dissemblables. Le collage "de" citations rassemble ces deux opérations, et porte à son maximum d'intensité la conjonction explosive du Même (l'énoncé répété) et de l'Autre (la différence entre énoncés) 9 .

7 Sans doute un tel parti pris est-il ce qui menace le plus nos traditions : la citation ainsi comprise s'interdit, en se multipliant, tout effet de réminiscence. Cet effet ne se limitait pas à l'évocateur ou au descriptif : ce qu'il y a d'unique, d'involontaire et de déchirant dans la réminiscence réelle (c'est à Proust qu'on pense évidemment) a pu donner à certains passages de Malher ou de Berg une profondeur temporelle dont seule la citation était capable. C'est cette temporalité propre à la citation "romantique" qui disparaît dans le collage, et non pas son identification ou sa reconnaissance, toujours possibles, mais condamnées à tourner au réflexe ou au jeu de société ${ }^{10}$. De même, la pluralité des emprunts débouche sur l'abandon d'un autre registre : celui de l'ironie et de la distanciation, propres à certains néo-classicismes - et c'est dès lors l'option formaliste ou constructiviste qui devient impossible. Traditions menacées, enfin, dans le champ de la théorie musicale proprement dite, pour qui la citation a toujours été un 
mauvais objet, plus ou moins marginalisé. Une telle politique est repérable dès l'origine, et il n'est pas sans intérêt de ne la voir affleurer que dans les marges, ou les silences, des discours musicologiques les plus vénérables. Lorsqu'Hanslick écrit, par exemple, que "le style peut être compromis par une seule mesure qui, irréprochable en soi, ne s'harmoniserait pas avec l'expression de l'ensemble"11, il désigne, bien sûr, un dangereux point-limite, seuil à partir duquel tel fragment mal contrôlé va dévier de sa trajectoire et donner la pire impression : venir de l'extérieur. Or la citation assume positivement cette tendance disruptive. Lorsqu'Hugo Riemann ravale au rang de "curiosité" musicale "l'exécution simultanée de deux danses hétérogènes dans la scène du Banquet de Don Juán ${ }^{12}$, il s'agit de valider pour de bon la forme sonate comme prototype achevé de la Forme musicale en soi, à partir d'un subtil dosage entre "conflit" et "contraste". Or le collage suppose d'abord contraste et ensuite (éventuellement) conflit (oppositions provoquées, entretenues puis surmontées : toute une problématique du Récit). Nous avons rompu avec des systèmes musicaux, des schémas pré-établis : avons-nous oublié certaines façons de penser ? Il faut reconnaître à de telles pratiques la capacité de faire apparaitre comme en creux les partages et les exclusions nécessaires à la fondation - et à la survie - de toute théorie esthétique. Là réside leur aspect véritablement "critique"; non pas dans la contestation de tel ou tel concept engendré par la théorie, mais dans celle, plus souterraine, de leurs conditions mêmes de constitution.

Dès lors, ne retrouve-t-on pas là les enjeux de toute "avant-garde" ? On ne sait que trop comment s'accomplit cette critique et à terme, ce nihilisme : "pour l'avant-gardiste, le matériau relève de la documentation. Son activité ne vise qu'à détruire la vie de ce matériau et à l'arracher au système de fonctionnement qui lui confère sa signification. Là où le "classique" reconnaît et considère ce qui a une signification dans le matériau, l'avant-gardiste n'y voit qu'une marque sans valeur, autorisant une signification dans la mesure où elle lui est appropriée"13. Le détournement de fonction, voilà la grande affaire : mais comment accepter que rien ne sépare l'objet trouvé des années soixantedix de celui des années quinze ou vingt? On peut répondre ainsi: de nouveaux principes d'organisation viennent régir les fragments rapportés, et en limiter la totale "émancipation"; l'iconoclastie n'est plus le fait des collages contemporains; la réinsertion du fragment dans un tissu syntaxique plus vaste, voire "ouvert", sa participation à des projets cohérents (d'ordre politique ou expérimental) lui confère une nouvelle nécessité. Et si le danger reste grand de voir la pluralité des emprunts basculer, comme naguère les sons ponctuels, dans l'indifférenciée ${ }^{14}$, le collage peut toujours se ressaisir comme procès dialectique, reflet d'une réalité plus complexe, et non plus écho grinçant d'un monde dissocié ${ }^{15}$.

9 Toute cette analyse, pour exacte qu'elle soit, pêche par deux défauts, qui n'en font qu'un d'ailleurs : en privilégiant le modèle des arts visuels, elle se limite à une approche par trop entachée d'idéologie; en ne tenant pas compte du Temps (de ce que peut impliquer pour le Temps musical l'emploi d'entités appartenant fonctionnellement à d'autres temps), elle laisse peu ou prou au propos extra-musical la mission de faire sens, et la porte est ouverte à quelque rechute dans un "programme". Ici, 1'intention signifiante est supposée irriguer instantanément les faits musicaux qui s'y rapportent. Et du coup, l'interprétation idéologique oblige à une concession majeure: la récupération de la citation dans un tout polymorphe va de pair avec le fait "qu'elle n'abandonne pas son mode de fonctionnement", autrement dit ce pouvoir de renvoi que lui accordait encore l'époque précédente. Le besoin - trop humain - d'un ordre 
supérieur garant d'une signification conduit à réintroduire dans la particule égarée le sens qu'il lui avait d'abord fallu perdre pour qu'elle fût bel et bien "collée" à ses voisines. On le voit: souscrire sans réserve à cette thèse, c'est manquer ce que la citation-collage (nommons-la ainsi) a de plus précieux, à savoir ce qui se dessine depuis le début, son caractère paradoxal. Cette technique va plus loin que l'oubli avantgardiste de la seule fonction, elle requiert un oubli de la mémoire elle-même, un peu au sens où l'oubli des relations aura autorisé, grâce à Cage, l'écoute des "sons eux-mêmes". Mais un tel oubli ne se commande pas: dans le cas de la citation, il ne s'agit pas de se défaire d'un savoir, mais de toute une nébuleuse de réactions et de comportements liés au fait même de répéter, plus qu'au fait de "renvoyer à". Le sens de la citation est à chercher dans la force de travail qui déplace ${ }^{16}$, le "borrowing" - et cette force-là ne se rend pas si aisément disponible. Ainsi est-il aussi difficile de neutraliser un matériau pré-organisé que de lui (re)conférer une signification après-coup, comme l'a bien vu Brian Ferneyhough : "Stravinsky, dans ses collages et ses pièces néo-classiques, a ignoré totalement le contenu émotionnel original de la musique, ce qui est honnête. Alors que de jeunes compositeurs d'aujourd'hui semblent d'une part vouloir emprunter ces éléments sonores, en faire ce qu'ils veulent, et en même temps (...) dire : "bon, j'essaie d'écrire de la musique réellement expressive et communicative comme un tel l'a fait" (...) et c'est absurde" ${ }^{17}$.

10 Interpréter le collage à partir de la citation, comme on vient d'être amené à le faire, semble bien impliquer un dépassement de la "référence" : or tel fut bien le cas aussi dans le courant de ces mêmes années soixante-dix. Un concept-vedette s'est alors imposé, qui permettait de dépasser toutes les contradictions de tout à l'heure : celui de "Texte". Car le "Texte" rejoignait par au moins deux voies le collage musical: en répudiant largement tout un ancrage référentiel ${ }^{18}$, (et à fortiori idéologique) il se rapprochait du musical en général comme art non représentatif, ne renvoyant qu'à luimême, ou comme le dit Clément Rosset, "sans double"; et par un autre détour, en se laissant définir comme "patchwork", "greffe", "hétérogénéité", "transcription de transcription" 19 ... il permettait de penser tout texte musical sur ce modèle, et en premier lieu, bien sûr, ceux qui se manifestaient ouvertement comme pluriels, greffés, tissés de citations. On voit la circularité de l'analyse: si Texte et collage sont originairement "la même chose", les musiques "à citations" ne font que ratifier ce que toute musique est déjà. Leur compréhension devient immédiate, transparente, mais demeure sans conséquences ${ }^{20}$.

11 Le bénéfice est néanmoins immense: grâce au "Texte", nous sommes (provisoirement?) dégagés des impasses de la référence, et renvoyés (musicalement) à la dépproppriation, au "flux", à la jouissance ${ }^{21}$. Cela conduit d'ailleurs à réinterpréter les positions d'un compositeur peu suspect de muséophilie, mais plus que quiconque habité par le Texte: il n'est pas sûr que Boulez soit resté tout à fait étranger à une certaine forme de pensée citationnelle, bien dissimulée il est vrai. On peut suivre ici le philosophe Pierre Missac qui prononçait en 1975 un "Eloge de la citation" ${ }^{22}$, en des termes que n'eût pas désavoués l'auteur d'Explosante fixe. Que trouve-t-on, en effet, à l'horizon de la citation? "L'Aphorisme" et "Le Livre" (dans une perspective ouverte par Walter Benjamin). Sur le versant aphoristique (pour nous: versant Webern): les pratiques de ce type "participent en l'espèce de la tendance contemporaine vers la brièveté, de l'hostilité radicale au développement dont témoignent la musique et, au sein de la théorie littéraire, les idées de Blanchot ou de Foucault". La puissance de la "rupture" - par quoi advient l'“éclat" de la citation - peut aussi profiter à l'œuvre 
entière, pour peu qu'elle s'astreigne à une extrême concentration sur soi : la pierre précieuse se refuse à servir d'objet trouvé. En même temps, la porosité que lui confère le silence la rend pour ainsi dire soluble dans l'espace. La tension entre "assimilation" et "dissimilation" qui travaille la citation devient la caractéristique de l'organisme musical tout entier ${ }^{23}$.

Sur l'autre versant, le Livre prend en charge, à grande échelle, l'hétérogène: "parenthèses et italiques", rêve de faire "alterner Bruckner et Webern", intérêt pour la jungle de Berg, tout cela renvoie bien, chez l'intéressé, et de son aveu même, à une certaine "hantise" de la multiplication des degrés. Entre le "collage" (au sens où nous l'entendons) et le "livre" (de Pound à Joyce, et au-delà) la différence est plus de niveau que de nature. Et n'est-il pas savoureux de voir, dans tel interview à propos de Répons ${ }^{24}$, la " $4 \mathrm{X}$ " faire bon ménage avec les paperolles de Proust?

13 Sans doute est-on passé bien vite du Texte au Livre, mais importait-il ici de les différencier? Car c'est aussi leur solidarité qui, globalement, aura constitué une grille de lecture irremplaçable pour la musique contemporaine, et plus spécifiquement la possibilité d'une connivence de fait entre les musiques citantes ou collées et la théorie littéraire dans ses avancées les plus radicales.

Tout ce qui précède n'épuise pas, loin de là, le sens d'une démarche esthétique que partagea toute une génération de grands citateurs : ce que citation et collage peuvent devoir à la technologie, de l'antédiluvien magnétophone ${ }^{25}$ aux actuels échantillonneurs, devrait être aussi médité, pour autant que les implications esthétiques de la répétition y rencontrent de manière originale celles de toute "reproductibilité technique". On sait que c'est un rapport différent à la technologie qui consacre la différence entre avantgardes (au sens où nous l'envisagions plus haut) et néo-avant-gardes (au sens de Gianni Vattimo ${ }^{26}$. Et ce clivage n'affecte pas que les élites artistiques. Si les collages des années soixante-dix sont restés, de par leur caractère "expérimental", le lot des professionnels et des spécialistes, l'étonnante vogue médiatique des "transcriptions", remarquée par Boucourechliev dix ans plus $\operatorname{tard}^{27}$ et qui se poursuit aujourd'hui, apparait un peu comme leur retombée au niveau de la consommation de masse: une extension du marché de la référence, qui demande, bien sûr, une tout autre analyse. De même, on peut trouver dans un phénomène comme celui de la "House Music", l'accomplissement du principe citationnel, pour ainsi dire dénudé par la technique - à moins que la technique ne s'incarne dans la citation. Cette dernière passe alors d'un statut à un autre : de "procès dénonciation", "trace du désir dans l'écriture", elle devient fait de langue, c'est elle la loi d'une musique où l'ancien "fragment", atomisé à l'extrême, se déplace à la vitesse de la lumière et se connecte instantanément à n'importe quel autre ; c'est elle qui finit par fonder la syntaxe au lieu de la subvertir. Ainsi peut-être verra-t-on, dans cet ultime avatar de la référence, et en dépit de sa trivialité, se dessiner la tendance fondamentalement utopique (et non plus seulement paradoxale) de tout collage citationnel. 


\section{NOTES}

1. Selon l'expression de Jean Baudrillard.

2. Dahlhaus, Carl : Plaidoyer pour une théorie actuelle de la musique, in Quoi, quand, comment : la recherche musicale, Paris, Bourgois, 1985..,

3. Une approche en termes "d'influence" semble bien, de la même façon, dérisoire ; une étude de Charles Rosen (Influence: plagiarism and inspiration, 19th century Music, IV, 2, 1980) permet de mesurer la distance qui nous sépare aujourd'hui de Brahms imitant Beethoven dans le Final du Premier Concerto pour piano.

4. "La meilleure façon d'analyser et de commenter quelque chose est, pour un compositeur, de faire quelque chose de nouveau en utilisant le matériau de ce qu'il veut analyser et commenter. Le commentaire le plus fructueux d'une symphonie ou d'un opéra a toujours été une autre symphonie, un autre opéra. (Berio, Luciano : Entretiens avec Rossana Dalmonte, Paris, Lattès, 1983, pp. 145-146.).

5. Cf. Metzger, Heinz Klaus : "Musique, pour quoi faire ?" in Musique en Jeu No. 1, 1970, a propos de la "Manifestation nunique" du 10 mai 1968 au Centre de Musique de Paris.

6. Par exemple: Nattiez, Jean-Jacques : Fondements d'une sémiologie de la Musique, Paris, 10/18, 1976. La citation n'y apparaît fugitivement qu'à titre d'illustration d'une typologie (signe, signal, symbole...).

7. Comme l'explicitait bien le Stockhausen d'Hymnen: "Les hymnes nationaux sont la musique la plus connue que l'on puisse imaginer (...) Si l'on intègre de la musique connue dans une composition de musique inconnue et nouvelle, on entend particulièrement bien de quelle manière elle a été intégrée : non transformée, plus ou moins transformée, transposée, modulée, etc." (Stockhausen, Karlheinz : Hymnen, in Contrechamps, No spécial, Paris 1988, p. 103.).

8. C'est à partir d'une réévaluation de Stravinsky qu'Henry Pousseur, par exemple, pourra rêver d'une vaste "étape synthétique", d'un nouveau mode d'organisation qui intégrerait aussi bien les "matériaux historiques": "il faudra attendre le dépassement des exclusives et des exclusions propres à l'atonalisme viennois, le dépassement d'une définition trop simplement antithétique et oppostionnel du nouvel ordre sonore ; il faudra attendre la recherche (sinon la découverte) d'un système harmonique beaucoup plus général, permettant d'intégrer l'harmonie chromatique des Viennois aussi bien que les diverses harmonies plus consonantes de notre histoire, en passant par toutes les tentatives de la musique moderne pré-sérielle et en ouvrant la voie tant à l'intégration des harmonies extra-européennes qu'à la mise à jour (...) de possibilités harmoniques nouvelles par leur matériau même..." (Musique en Jeu No. 4, 1971).

9. La superposition d'œuvres "pouvant aller ensemble" (par exemple, chez Cage, Atlas Eclipticalis et Winter Music) relève d'une autre problématique, bien qu'on puisse aussi parler de "collage".

10. Comme le craignait alors Dominique Jameux: "La citation, aujourd'hui le collage, apparaissent tout à la fois comme l'aveu d'une trace et la tentative d'une conjuration (...). A se vouloir - parfois - attentat à la propriété intellectuelle bourgeoise, (ne risque-t-elle pas surtout) d'établir la connivence des bourgeois cultivés ?" (Musique en Jeu No. 4, 1971).

11. Hanslick, Edouard: Du Beau dans la Musique, Paris, Bourgois, 1987.

12. Riemann, Hugo : Les éléments de l'Esthétique musicale, Paris, Alcan, 1903.

13. Sonntag, Brunhilde: Untersuchungen zur Collage Technik in der Musik des 20 Jahrunderts, Regensburg, Bosse, 1977. Dans ce qui suit, nous nous inspirons du débat que l'auteur mène avec Peter Bürger (Theorie der Avant-garde, Frankfurt, 1974).

14. Et même l'indéterminé: Brunhilde Sonntag rapproche "l'incertitude du matériau à employer" dans le collage de "l'absence de décision de l'artiste" qui prévaut dans l'aléatoire.

15. Cf. Hauser, Arnold : Socialgeschichte der Kunst und Literatur, Munich, 1972. 
16. Nous empruntons ici à Compagnon, Antoine; La seconde Main, Paris, Seuil, 1971.

17. Ferneyhough, Brian : Entretien avec Philippe Albèra, Contrechamps No. 3, 1982.

18. Nous ne pouvons mieux préciser notre pensée qu'en rappelant les réserves plus récentes de Paul Ricœur au sujet de cette "poétique contemporaine, qui récuse toute prise en compte de la référence à ce qu'elle tient pour extra-linguistique, au nom de la stricte immanence du langage littéraire à lui-même. (...) cette poétique s'emploie à tenir pour un simple effet de sens ce qu'elle décide, par décret méthodologique, d'appeler illusion référentielle". Ricœur, Paul : Temps et Récit, Paris, Seuil, 1984, tome 1.

19. La théorie du Texte trouve son ancrage dans une Philosophie de l'écriture: rappelons seulement quelques formules (très... parlantes) de Jacques Derrida : "La structure même de l'écrit est indissociable de cette force de rupture avec son contexte" qui caractérise le signe (Grammatologie, p. 378) ; “Telle écriture qui ne renvoie qu'à elle-même nous reporte indéfiniment et systématiquement à une autre écriture" (Dissémination, p. 229) ; “Ecrire veut dire greffer. C'est le même mot (...) La greffe ne survient pas au propre de la chose. Il n'y a pas plus de chose que de texte original (...) L'hétérogénéité des écritures, c'est l'écriture elle-même, la greffe" (Dissémination, p. 395), etc.

20. Que devient la citation, si "écrire, c'est toujours réécrire, et ne diffère pas de citer", et si "le geste citationnel n'est alors que la désignation du fonctionnement du texte en général”, comme l'entend François Escal ? (Le Compositeur et ses modèles, Paris, PUF, 1985).

21. Missac, Pierre : Eloge de la Citation, Collectif Change, No. 22, Mars 1975.

22. Ivanka Stoïanova: "La distribution habile des fragments sur un support uniformisant (...) relève souvent (...) d'un excédent absolu de plaisir dans la manipulation des matières qui affirme la multiplicité”... (Berio : Chemins en Musique, La Revue Musicale, No. 375376-377, 1985).

23. L'aboutissement de cette logique se trouvant dans l'Opus 28 de Webern, où citation et matériau sériel se confondent. Les expressions "assimilation et dissimilation" viennent de Kühn, Clemens: Das Zitat in der Musik der Gegenwart, mit Ausblicken auf bildende Kunst und Literatur, Hamburg, 1972.

24. Libération, 1987.

25. Souvenons-nous: "On retrouve ainsi le second aspect du magnétophone, qu'on avait tout d'abord pris pour une machine à faire des sons, à les assembler, à créer des objets nouveaux, voire de nouvelles musiques. C'est aussi, c'est d'abord (pour la recherche) une machine à observer les sons, à les “décontexter", à redécouvrir les objets traditionnels, à réécouter la musique tradionnelle d'une autre oreille, d'une oreille sinon neuve, du moins aussi déconditionnée que possible." (Schaeffer, Pierre : Traité des objets musicaux, Paris, Seuil, 1966).

26. Vattimo, Gianni : La Fin de la Modernité, Paris, Seuil, 1987. L'idée importante, héritée de Walter Benjamin, est celle du caractère constitutif de la reproductibilité.

27. Boucourechliev, André : "Le corps piano" in Le Monde, 1986. A propos de Liszt : "Son plaisir est celui du second degré, certes; et en ce sens, la vogue actuelle des transcriptions peut être assimilée au succès du post-modernisme...". 


\title{
Rejet de la pensée du matériau ?*
}

\author{
Carl Dahlhaus
}

Traduction : Vincent Barras

1.

Le slogan "fétichisme du matériau" est presque aussi ancien que les mouvements contre lesquels il est dirigé. Peu importe ce que l'on entendait par "matériau" 1'histoire "sédimentée" dans les sons, au sens d'Adorno, et dont la "tendance" fut portée à son terme par la musique sérielle des années 1950, ou le matériau des bruits, dont 1'aptitude musicale voire anti-musicale fut testée dans les années 1960-, on était toujours prêt à user du vocable péjoratif pour exprimer sa défiance, soit parce qu'on était resté en deçà de ce qui justement passait pour la pointe du développement, soit parce qu'on 1'avait déjà dépassée - bien qu'il n'ait pas toujours été facile de distinguer les deux situations ; ce qui semblait régression pouvait en effet se révéler inopinément être anticipation d'une part du futur.

2 Si l'on prend le concept de fétichisme au sérieux, au lieu d'en mésuser sans réfléchir comme d'une injure, il signifie alors - du moins dans la terminologie de Marx, d'où il a passé dans le langage familier des compositeurs - que les hommes vénèrent une idole qu'ils ont eux-même créée. Le sujet se soumet à une puissance, dont l'objectivité n'est que pure illusion, et dont la substance provient en réalité du sujet lui-même.

3 L'instance au nom de laquelle on proteste contre le fétichisme du matériau est par conséquent la subjectivité du compositeur, qui se refuse à accepter une loi prescrite par la nature ou par l'histoire et décidant de ce qui doit être fait ou ne doit pas être fait. Exiger qu'on se soumette sans recours à un "diktat" du matériau - compris comme le sédiment de toute une histoire de la composition et de la réception à l'intérieur des sons et des bruits ou des relations qu'ils entretiennent l'un avec l'autre - est ressenti comme intolérable.

4 La révolte du sujet contre un processus qu'on peut interpréter comme objectivation nécessaire, mais aussi comme aliénation fatale - et il faudrait cesser une fois pour toutes de se jeter à la face les interprétations les plus diamétralement opposées, et réfléchir plutôt aux critères grâce auxquels il est possible de déterminer quand et comment on passe de l'objectivation à l'aliénation - est un thème si ancien et si rabâché 
de la philosophie qu'il ne vaut pas la peine de puiser dans l'arsenal des arguments qui ont servi à alimenter la dispute depuis presque deux siècles, à moins qu'une raison d'actualité y pousse : on ne devrait faire appel à la philosophie que lorsqu'on ne peut s'en sortir sans elle. De fait, il semble néanmoins qu'on rende au mieux justice aux mouvements étiquettés - signe d'un malentendu selon leurs représentants - comme "Nouvelle Simplicité", voire "Nouvelle Tonalité" en les concevant comme des tentatives de restitution de la subjectivité en tant qu'instance décisive d'un contexte de signification sonore ${ }^{1}$, cette subjectivité que le compositeur aimerait sauvegarder des dangers encourus du fait de son inscription sociale en la réalisant sur le plan musical justement. Discuter du rejet de la pensée du matériau et des implications, ressenties comme gênantes, du concept de matériau revient donc en même temps à s'interroger sur les possibilités et les difficultés d'une expressivité subjective non dissimulée. Quiconque ne veut pas parler de sujet, fût-ce par timidité, par méfiance ou par désespoir, est contraint de se taire sur ce qui se passe aujourd'hui dans la musique.

2.

5 En procédant à un examen rétrospectif des années 1950, il faudrait même se demander si la conception de la musique sérielle comme accomplissement d'une "tendance du matériau" pour ainsi dire sans sujet, objectivement historique était en fin de compte aussi concluante qu'elle paraissait, non seulement aux commentateurs, mais aussi aux acteurs de l'histoire de la composition. Et, dans l'argumentation que nous cherchons à exposer, ce pas en arrière n'est aucunement digression superflue.

Prétendre que dans les oeuvres dodécaphomiques de Schoenberg il y aurait une contradiction entre la structure des hauteurs avancées et la rythmique traditionnelle, désuète, contradiction que seule la musique sérielle aurait résolue, est erroné, car aussi bien la structure dodécaphonique que la rythmique non-sérielle servent le même but: rétablir les "grandes" formes instrumentales autonomes sous les conditions de l'atonalité. Ce n'est pas l'analogie des paramètres, mais le fait qu'ils se complètent fonctionnellement qui constitue dans la pensée musicale de Schoenberg le principe de régulation des relations entre structure de hauteurs et structure de durées. Dans les années 1920, le dodécaphonisme se trouve donc dans une autre problématique que trois décennies plus tard, et c'est ce qu'on se fixe comme but compositionnel qui détermine les questions à poser ou à ne pas poser.

7 Si par conséquent la contradiction résolue par la musique sérielle ne s'est constituée qu'avec sa résolution - elle n'existait pas dans l'oeuvre de Schoenberg -, on peut alors aisément substituer à la conception de la nouvelle musique comme accomplissement de "tendances du matériau" une autre conception, celle que proposait en 1937 déjà Ernst Krenek, en parlant de la "liberté de poser des axiomes" dont doit disposer le compositeur. Selon Krenek, la musique en tant qu'art repose initialement sur des axiomes qui ne sont posés ni par la nature ni par l'histoire, mais par le compositeur, dont les intentions esthétiques décident de ce qui doit constituer, du point de vue technique compostionnel, le contexte de signification.

8 Pour illustrer le concept d'axiome, il suffit de rappeler que les "degrés de combinaison" de l'ensemble des différentes sonorités sont certes déjà tracés dans la nature de la perception humaine, mais que c'est au compositeur qu'il incombe de choisir entre les différentes possibilités de développer des faits musicaux à partir des conditions acoustiques préalables. Les intervalles doivent-ils être divisés en deux ou - comme au $\mathrm{XIV}^{\mathrm{e}}$ siècle - en trois classes, la technique compositionnelle doit-elle respecter les 
degrés de combinaison ou - comme dans la quarte sur la fondamentale du contrepoint strict - les contrecarrer, cela dépend exclusivement des buts que s'est fixés le compositeur, à qui seul il incombe de choisir les axiomes techniques-compositionnels correspondant à ses idées esthétiques.

9 La "liberté de poser des axiomes" proclamée par Krenek ne signifie rien moins que c'est au sujet compositionnel, et non à une contrainte objective exercée par la nature ou par l'histoire de la musique, que revient le dernier mot. Et si l'on fait siennes les prémisses de Krenek, la musique sérielle apparaît non pas comme la conséquence nécessaire et inéluctable d'un problème troublant et irrésolu provenant du dodécaphonisme schoenbergien, mais comme un axiome posé en toute liberté par les compositeurs des années 1950, ceux surtout dont les questions ne pouvaient guère trouver de dénominateur commun avec celles de Schoenberg trente ans plus tôt.

Là où apparemment un diktat du matériau traçait le chemin que devaient suivre les compositeurs - s'ils ne voulaient pas s'exposer au risque de produire de l'insignifiant-, était en réalité à l'oeuvre une subjectvité qui, bien que dissimulée derrière l'illusion d'une "nécessité historique", n'avait aucunement disparu. La légitimité esthétique d'une oeuvre telle que Gruppen ne repose pas sur la rigueur avec laquelle étaient tirées les conséquences historiques inévitables; au contraire, la plausibilité esthétique du produit engageait à reconstruire les conditions historiques préalables de telle sorte qu'elles semblent aboutir au résultat.

11 Le rejet de la pensée du matériau, que l'expression "fétichisme du matériau" résume en un slogan, sans que tout d'abord l'instance opposée ne soit clairement constituée - un rejet devenu plus tard dans les années 1970 tendance dominante - ne veut donc pas dire que l'objectvité ait été remplacée par la subjectivité, mais plutôt qu'une subjectivité manifeste a remplacé une subjectivité latente. "L'histoire" - au singulier : un singulier extrêmement problématique - s'est avérée, pour le dire sans ménagements, être un mythe. En tant qu'instance qui trône au dessus des actions du sujet, elle a perdu son pouvoir sur la conscience, instauré au XIX ${ }^{e}$ siècle par Marx et Hegel. Personne ne nie bien entendu qu'il existe des contextes, c'est à dire des "histoires" - au pluriel : mais depuis, nous croyons savoir de "l'histoire" - au singulier - qu'elle n'existe pas. "L'histoire" n'est donc pas plus un terme d'historiographie que "la nature" n'est un terme de science naturelle.

3.

12 Si donc, par cet examen rétrospectif des années 1950, le concept adornien de matériau, qui prit dans la Philosophie de la Nouvelle Musique la place de 1'esprit objectif, gouvernant l'histoire, se révèle être une prémisse problématique, du fait qu'il réprime ou rejette le moment de choix subjectif souligné par Krenek, en revanche, l'inclination actuelle pour une subjectivité non dissimulée, sans réserves, qui ne veut rien savoir de tendances objectivement contraignantes, contient un motif implanté dans la pensée et dans le sentiment musical par John Cage. En se refusant à obéir à un diktat de 1'histoire, les compositeurs succombaient dans le même mouvement à la tentation de poursuivre une idée alambiquée de la nature musicale, confondaient autrement dit une fois de plus les paradigmes esthétiques de nature et d'histoire, bien que le nouveau concept nature n'ait eu que peu à voir avec l'ancien, propagé en dernier ressort par Hindemith. Et la différence dont nous discutons peut s'illustrer de la manière la plus simple par les concepts de matériau et de matière : le concept adornien de matériau est en effet une catégorie historique, alors que l'idée cagienne de matière est une catégorie de nature. 
D'une part, forme et matière sont, dans la pensée européenne forgée par Aristote, des concepts relatifs : la brique est la forme du morceau d'argile, la maison est la forme des briques, le village est la forme des maisons. D'autre part - et la deuxième caractéristique est décisive-, forme et matière sont reliés l'un à l'autre en tant que corrélats : l'un n'existe pas sans l'autre, et la matière en soi - bien que le terme de matière suggère le concret - est une abstraction au même titre que la forme en soi. Mais 1'idée de se saisir de la "matière première" dans sa manifestation acoustique, materia prima sonore, forme précisément le centre autour duquel gravitent les événements musicaux ou anti-musicaux que projette John Cage avec une inventivité intarissable. Les moyens avec lesquels Cage tente malgré tout de faire apparaître et de rendre perceptible, ne serait-ce qu'un instant, ce qui est une abstraction réellement vide, sont ceux d'une annulation et d'une destruction acharnée de l'ensemble des moments formels, qui, comme le prétend Cage, barrent la route à la "vraie nature" du sonore. Il devrait être évident que la seule expérience, s'il en est une, dans laquelle on puisse accéder à la "matière première" est une expérience mystique. Et la mystique, dans un sens tout à fait sobre et précis, et aucunement nébuleux, est bien ce sur quoi repose en dernière instance le désir de destruction de Cage, tant il est vrai que les grands destructeurs de l'histoire de la politique ou des idées ont souvent été des mystiques passionnés.

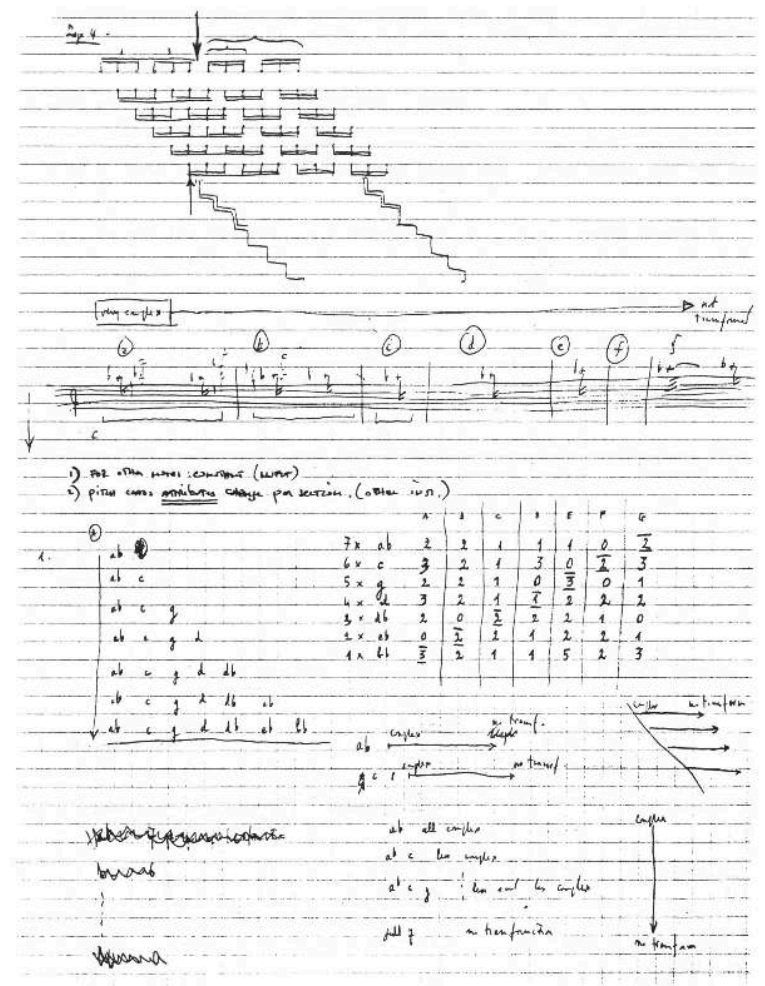

14 Toutefois, les tendances que suit Cage sont profondément antinomiques et exposées en conséquence aux malentendus et aux simplifications, sans lesquels d'ailleurs l'influence très large qu'il a exercée n'aurait guère été pensable. Le fait que les sons et les bruits que Cage présente ou laisse surgir ont été détachés de leur contexte pragmatique d'origine, de sorte qu'ils n'ont pas, comme dans le quotidien, la fonction de signes et de symptômes de processus survenus dans le monde extérieur, mais qu'ils forment un "monde en soi" acoustique, signifie tout simplement que l'anti-art de Cage et l'art, dont il recherche la destruction, ont en commun le moment de l'“abstraction esthétique". 
Les événements acoustiques sont, pour employer un stéréotype, "dépragmatisés", et par là "esthétisés". Cependant, et l'on retrouve là un trait dadaïste, le contexte extérieur-pragmatique ne doit pas se substituer à un contexte intérieur-esthétique ; au contraire, c'est le principe du contexte lui-même - du contexte de signification acoustique - qui est l'objet d'une agression visant en dernier lieu, comme on l'a dit, à l'expérience mystique d'une "pure matière". Mais du fait que la constitution d'un contexte intérieur-acoustique, qui établirait au moins rudimentairement un caractère artistique, est interdite par la ruse obsessionnelle de la non-raison, les processus sonores peuvent alors facilement verser dans le quotidien - d'où la suspension de l'abstraction et l'isolement esthétique : un quotidien toutefois qui n'est pas un monde pragmatique, guidé par des buts, mais un monde agressivement vain, écartant toute idée de signification et de but. Ce que Cage recherche, c'est, pour parler avec Kant, une expérience sans concept et désintéressée.

4.

15 Prétendre que la composition de sons et de bruits des années 1960 ait été dans son ensemble portée par l'intention cagienne de s'immerger dans la nature du sonore serait à vrai dire une exagération grossière. La coïncidence de la destruction et de la mystique, qu'il est difficile d'obtenir et de fixer, fut remplacée chez la plupart des compositeurs ayant succombé à l'emprise cagienne par un penchant plutôt inoffensif pour la découverte et l'assemblage de sons et de bruits inconnus, ou du moins inhabituels à l'intérieur d'un contexte esthétique. Considérant la simplification qui en résulta, il n'est pas étonnant que la composition de sons et de bruits - qui de plus souffrit esthétiquement de sa récupération en quincaillerie musico-pédagogique - ait rapidement abouti à un état d'épuisement, oracle fatidique de presque toutes les tendances artistiques récentes initialement marquées par leur radicalité (desquelles se distingue d'autant plus singulièrement le Jugendstil redécouvert, dont la durée dépasse de loin celle de l'original).

16 A partir de la problématique de la musique post-sérielle, on peut toutefois dériver etrendre compréhensible, du moins à titre d'essai et d'hypothèse, la tendance qui reçut tout d'abord le nom équivoque et trompeur de "nouvelle tonalité". La composition de sons et de bruits partageait avec la nouvelle musique, dont elle représentait l'antithèse, une prémisse importante, et même, comme il s'avère rétrospectivement, fondamentale: le présupposé que la distinction entre propriétés centrales et périphériques du son, qui dans la musique européenne avait appartenu des siècles durant à ces évidences solidement enracinées, soustraites à la réflexion, devait être déclaré sans valeur si l'on voulait qu'un progrès musical soit encore possible. Intensité et timbre ne devaient plus être traités en subordonnés, uniquement destinés à colorer et à mettre en valeur la structure de hauteurs et de durées, mais comme des paramètres indépendants et égaux en droits. On peut même prétendre sans exagérer que dans la composition de sons et de bruits, c'est justement dans les propriétés sonores passant auparavant pour secondaires que l'on essayait de réaliser et d'exploiter des découvertes.

17 Le fait que dans les années 1970 l'exploration post-sérielle de ce qui était périphérique dans la musique traditionnelle, et devenu soudain centre de l'attention, ait mené à un rétablissement de l'expressivité, antithèse emphatique de la pensée structurelle initiale, n'est pas si étonnant qu'il n'y paraît tout d'abord. Car entre les propriété sonores secondaires, promues au rang de primaires, et le moment d'expression de la 
musique, règne indubitablement une étroite affinité, de même qu'à l'inverse les paramètres primaires, désormais réduits à une position secondaire, continuaient d'être considérés et traités comme ayant une fonction de structuration. Dans la musique traditionnelle, hauteur et durée étaient caractérisées par le fait qu'on pouvait déterminer structurellement la différence entre essence et existence: la fonction harmonique et métrique d'un accord, c'est-à-dire sa signification musicale à la différence de sa simple facticité acoustique, est un moment structurel. Par contre, dans l'intensité et dans le timbre, la "deuxième couche" qu'ils contiennent en plus de la couche acoustique, est moins déterminée structurellement qu'expressivement ou que sur le plan de la couleur. Le rigorisme structurel de la musique sérielle, immédiatement évident en ce qui concerne la hauteur et la durée, devenait pour l'intensité et le timbre une sorte de coup de force, qui voulait imposer de la structure en un sens précis et sévère, y compris là où les paramètres ne convenaient guère en soi à un tel traitement. C'est pourquoi, même si le revirement apparaît au premier abord comme une abrupte rupture de continuité, il est nullement inconcevable que la musique récente, tout en tenant ferme à la restitution de l'expressif, ait toutefois, comme il semble, renoncé depuis longtemps à l'idée d'une équivalence structurelle des paramètres, sans que cela ait été clairement explicité. Sous le signe d'une subjectivité s'avouant à nouveau telle, les moments d'expressivité des compositions de sons et de bruits sont accentués et poussés à l'extrême : ce sur quoi on "menait des recherches" dans les années 1960, comme si le matériau se suffisait à lui-même, fut utilisé dans la décennie suivante comme un moyen à des fins expressives. Mais la structure musicale, pour ainsi dire colonne vertébrale de la forme, est à nouveau livrée à un primat de la hauteur et de la durée. Et s'il n'est pas possible de parler d'une "nouvelle tonalité", dans la mesure où l'on prend les concepts au sérieux et qu'on n'en mésuse pas comme de simples étiquettes, on ne peut d'autre part se méprendre sur la propension générale, mais extrêmement différenciée dans les différentes manières de réalisation, à restituer la différence entre essence et existence, entre fonction musicale et fait acoustique, à l'intérieur des paramètres qui étaient initalement les fondements de la structure.

5.

18 Au rejet de la pensée du matériau s'ajoute ces dernières années une indifférence croissante à l'égard de la théorie, ce que l'on peut certes comprendre après la manie théorique des années 1950 et 1960 - après la prolifération d'auto-commentaires déguisés en théories générales-, mais qui, en tant que réaction, est portée comme toutes les réactions à l'exagération, et peut amener à de fatales conséquences. Le penchant de certains jeunes compositeurs à éviter avec une naïveté feinte une tendance évolutive que Jürgen Habermas a désignée du terme de "loi de la réflexion en progrès" est finalement vain, tout séduisant qu'il paraisse au premier abord, en un temps de lassitude générale de la réflexion. Et pour ne pas se fourvoyer en un cul-desac auquel aboutit presque obligatoirement une pratique aveugle, on ne peut apparemment éviter de récapituler les exigences soulevées par la théorie pendant des décennies et, sous les conditions modifiées du présent, les mettre une fois encore à l'épreuve.

19 Le principe du "work in progress", une des idées maîtresses des deux décennies entre 1950 et 1970, était inséparablement lié à la théorie, dans la mesure où l'histoire de la composition était construite - certes non pas uniquement par les commentateurs, mais aussi par les acteurs mêmes - selon le modèle de l'histoire des sciences : une histoire où 
les problèmes amènent à des solutions, qui à leur tour révèlent de nouveaux problèmes, de sorte qu'il faut à nouveau rechercher des solutions, et ainsi de suite en un processus dont la dialectique semble en principe ne pas avoir de terme. Mais si ce n'est plus, comme au XIX et jusqu'au début du XX ${ }^{\mathrm{e}}$ siècle, l'oeuvre achevée, refermée sur soi, mais au contraire le produit provisoire, chargé de contradictions irrésolues et simultanément ouvert vers le futur qui forme le véritable objet de l'effort compositionnel, la réflexion théorique apparaît dès lors inévitable, elle qui réalise la médiation intellectuelle entre les étapes précoces et tardives du "work in progress" individuel ou même intersubjectif : une réflexion qui tend à la formulation verbale et la publicité - à la diffusion vers le public intéressé par la nouvelle musique. Car, en tant que simple document d'une phase de développement, une oeuvre musicale qui n'aimerait plus être oeuvre au sens emphatique du terme et dont les exécutions la présentent pourtant aux auditeurs comme si elle valait en tant que telle n'est compréhensible que grâce à un commentaire explicatif décrivant le contexte du "work in progress". L'œuvre en tant que fragment esthétiquement présent d'une chaîne évolutive non-présente a besoin de la réflexion théorique, de la réflexion publiée, afin d'être reçue comme on veut qu'elle le soit.

En une époque où l'on a depuis longtemps abandonné la croyance en l'inspiration du $\mathrm{XIX}^{\mathrm{e}}$ siècle - à l'exception de sectes qui, même si elles deviennent majorité, ne cessent d'être des sectes - il n'est guère besoin de répéter qu'au fond tout processus de composition, y compris le plus primitif, inclut une réflexion aussi rudimentaire soitelle. Il faut toutefois distinguer entre théorie "implicite", qui comprend la composition comme pensée "en" musique, et théorie "explicite", qui se manifeste comme pensée "sur" la musique. (Il va de soi qu'avec le concept de théorie, on n'entend pas ici la propédeutique technique musicale qui usurpe le terme dans l'éducation musicale). Et ce dont il est question n'est pas la pensée musicale au sens absolu, la "logique musicale" comme disait Schoenberg, mais la mesure dans laquelle une pensée "sur" la musique remplit une fonction utile voire indispensable pour la pensée "en" musique inhérente à toute composition, ou au contraire représente un ajout superflu à une pratique compositionnelle autonome et seule décisive.

21 Pour ne pas étendre vainement la controverse entre utilité et inconvénients de la théorie pour la pratique, dont on peut prétendre sans exagérer qu'elle est menée depuis des siècles, de telle sorte qu'une continuation à l'infini est vraisemblable, le plus raisonnable semble d'étouffer les réminiscences historiques qui s'imposent spontanément et de partir plutôt une nouvelle fois du concept de subjectivité, qui s'est avéré être la catégorie dominante de la discussion esthétique actuelle. Il n'est dès lors pas besoin de longues explications pour comprendre qu'un sujet qui ne travaille pas à un objet, qui n'interagit ni ne communique avec d'autres hommes et qui ne possède pas un langage servant à la compréhension de soi-même et du monde extérieur est ce que Hegel nommait une "intériorité abstraite" : un "abîme vide", pour employer les mots de Schopenhauer, pour une fois en accord avec son adversaire. La subjectivité ne se constitue - que l'on me pardonne la trivialité anthropologique - qu'au moment de l'interaction productive avec un objet, de l'interaction agissante avec d'autres sujets et linguistique avec un environnement, qui, sans concepts organisateurs, serait un chaos de sensations - c'est-à-dire, selon les termes d'Aristote, au moyen de la poiesis, de la praxis et de la theoria. Mais si l'on admet que, dans l'activité compositionnelle, le travail sur un matériau, l'interaction avec les hommes, dont les attentes appartiennent aux prémisses du travail - peu importe qu'on les remplisse ou qu'on les contrarie-, et 
l'explication verbale de son propre agir et de ses propres expériences sont inextricablement liées, il devient alors évident que la théorie est toujours déjà impliquée dans la pratique musicale, et que par conséquent ce n'est pas l'alternative entre théorie et absence de théorie, si attrayante pour de nombreux compositeurs, qui reste ouverte, mais bien le choix fatal entre théorie consciemment projetée et différenciée et théorie négligée et primitive. Il en va de la pensée musicale comme de toute autre : on ne choisit pas, même si l'on prétend à la naïveté, entre philosophie et non-philosophie, mais entre bonne et mauvaise philosophie.

Il est bien entendu absolument légitime, en un moment historique où le produit musical individuel se présente à nouveau comme oeuvre isolée, refermée sur soi, et non comme étape d'un "work in progress", de renoncer à une théorie explicite et de s'en tenir provisoirement à une théorie implicite, que l'on porte de toutes façons en soi : s'il était auparavant nécessaire de suivre intellectuellement des développements en continuel chevauchement, tout en essayant, dans une salle de concert justement, de s'approprier esthétiquement un morceau du processus compositionnel individuel ou intersubjectif, appellé dans son ensemble "musique avancée", les compositeurs de la jeune génération ont apparemment suspendu cette nécessité. Il n'est cependant pas superflu d'évaluer dans quelle mesure la tentative de substituer à la pensée "en" musique une pensée "sur" la musique, fixée par le langage, est utile ou nuisible au compositeur. L'argument mendelssohnien selon lequel la pensée musicale serait trop différenciée pour que le langage y ait accès représente dans la discussion ancienne comme dans l'actuelle l'antithèse de celui d'après lequel une imagination musicale qui se soustrait ou tente de se soustraire en son principe à la stabilisation verbale ne peut éviter au bout du compte de devenir diffuse. Autant la théorie explicite est insuffisante dans cette dernière instance, autant elle est apparemment indispensable dans la première. Une aversion extrême pour la réflexion, qui craint de porter atteinte à la pensée "en" musique en utilisant des catégories par trop grossières finit par étioler ce qu'elle aimerait protéger. Le silence, qui se sent rassuré et confirmé dans la possession muette de ce qui seul est essentiel aboutit en fin de compte à une pure et simple pauvreté intellectuelle. Et autant on aimerait que dans ces prochaines années la phase sérielle et post-sérielle de l'excès de théorie ne se répète pas, autant sont précaires non seulement en musique - la léthargie ouverte et l'hostilité cachée accueillant aujourd'hui tout "effort du concept" qui tente de s'exprimer le plus précisément possible à l'aide du langage. Une subjectivité qui aimerait parler exclusivement à travers les sons, parce que les mots seraient superflus voire trompeurs, conduira peutêtre une fois à la décourageante impression qu'elle n'a plus rien à dire y compris à travers les sons.

6.

Il semble qu'au rejet de la pensée du matériau et qu'au repli dans le subjectif soit liée une restitution du concept d'oeuvre ou du moins un arrêt de la polémique envers celleci. La barrière dressée pendant des décennies entre matériau et oeuvre incite immanquablement, si l'on détruit l'un des termes, à rétablir son pendant. La problématique du concept de l'oeuvre, telle qu'elle est apparue dans la discussion de naguère, n'est toutefois aucunement résolue, et pour comprendre ce qu'une restitution peut signifier ou non, il faut être conscient du fait que, dans les années 1950 et 1960, la polémique contre l'idée du produit autonome, isolé, refermé sur soi, provenait de motifs et d'arguments hétérogènes et parfois incompatibles. 
dichis rudiments de substance utopique pour le futur, le passage de l'art à la praxis de vie devenait une exigence $d u$ jour, qu'il ne fallait pas différer, mais satisfaire ici et maintenant, fût-ce au prix d'échecs répétés. La conséquence - inutile de prétendre le contraire - fut un relâchement de l'exigence artistique à cause de ce que l'on appelait au sens emphatique "communication". Et Adorno, s'il vivait encore, déplorerait sans nul doute la "déprofessionalisation" des résultats, le déclin du métier qu'on ne peut aucunement justifier ni même excuser par la bonne volonté aveugle avec laquelle on sacrifiait le niveau d'artisanat à une fiction sociale. L'argument selon lequel, au milieu d'une situation générale menant inexorablement à la catastrophe, une transformation significative de l'art en praxis de vie ne serait pas possible, même dans des enclaves qui ne le seraient qu'en apparence, était d'une certaine manière tout prêt pour l'explication des problèmes esthétiques.

D'autre part, la thèse radicale selon laquelle seul le renversement de la totalité permettrait un changement profond de l'individuel et du particulier appartient aux prémisses qui empêchent ce qu'elles espèrent comme conséquences. L'art reste emmuré en soi-même, parce qu'une situation où il aurait la possibilité de pénétrer pour de vrai dans la praxis de vie n'est pas prévisible. La discussion sur le concept de l'oeuvre - ou plus exactement : sur le rapport entre l'oeuvre en tant que travail objectif 
et les catégories de la "communication" et de l'interaction", devenues les slogans de la polémique menée contre le concept d'oeuvre - est toutefois curieusement embrouillée, même si les deux parties se fondent, ou croient se fonder, sur des prémisses marxistes. Adorno était un marxiste tout à fait orthodoxe lorsqu'il insistait avec entêtement sur le primat du travail qui s'objective dans les oeuvres: l'idée d'une interaction interhumaine où le résultat apparaît secondaire et le processus en tant que tel primaire lui était, de même qu'à Marx, entièrement étrangère.

Il est vrai que la version extrême du concept d'autonomie esthétique avait été depuis longtemps abandonnée, même par Adorno, qui comprenait l'autonomie comme résistance contre la société décomposée et procurait par là, comme ses adversaires le lui reprochèrente, une bonne conscience sociale à l'"échappisme". Dans son expression la plus rigoureuse, qui domina autour de 1900, le concept d'autonomie ne voulait rien dire moins que l'art et la science, qui obtenaient le même statut idéel, sont des principes qui ne servent pas les hommes, mais qu'à l'inverse les hommes doivent justement servir s'ils prétendent être comptés parmi les hommes de culture. Depuis l'objection simple que ce qui est fait par l'homme doit être là pour l'homme afin de ne pas paraître superflu est devenue opinio communis : plus personne n'est prêt à honorer la "sublime indifférence du beau" célébrée par Schelling.

"Communication" est donc le slogan du jour. La relation avec la catégorie de l'oeuvre refermée sur soi, qui revendique d'être là pour elle-même, n'est toutefois, comme on l'a dit, pas aussi simple qu'elle le parait dans la dichotomie qui oppose un concept d'oeuvre aliéné et un concept de communication abstrait, c'est-à-dire confronte deux extrêmes figés, plutôt que de rechercher les médiations, qui sont à portée de main. D'innombrables groupes informels ont fait la douloureuse expérience de constater que la communication - l'idée à laquelle ils se cramponnent obsessionnellement - échoue toujours et reste abstraite lorsqu'elle s'épuise dans la tentative d'échanger une "intériorité vide", comme dirait Hegel. Elle a de toute évidence besoin, pour être substantielle, d'un objet servant d'intermédiaire dans l'interaction entre les sujets qui recherchent la communication; et c'est par une concentration commune sur une chose - une chose certes qui en vaille la peine - plutôt que par la tentative de la produire dans une immédiateté sans objet que l'on aboutira à une intersubjectivité qui rende aussi justice au sujet comme personne, et non pas simplement comme titulaire d'une fonction. Mais la musique réussit d'autant mieux à servir d'instance médiatrice qu'elle se refuse à relâcher voire à suspendre ses exigences internes à cause du but qu'elle veut atteindre: l'adaptation pédagogique est toujours nuisible non seulement matériellement, mais pédagogiquement aussi.

31 Si la communication doit donc éviter le risque de se fourvoyer dans le cul-de-sac de l'immédiateté abstraite et sans objet, la conception continuellement dénigrée selon laquelle une oeuvre musicale comme totalité achevée serait détachée du sujet producteur tout comme elle n'aurait au fond pas besoin d'un sujet récepteur dans la "sublime indifférence du beau" représente quant à elle un extrême de la métaphysique de l'art sur lequel même Arnold Schoenberg - qui n'avait pas craint les conséquences les plus radicales dans nombre de ses propos - ne voulut pas s'attarder sérieusement : il se considérait certes comme l'instrument d'une force agissant à travers lui, et méprisait le public, qui le lui rendit bien par son hostilité, mais il parlait, y compris en tant que personne, en musique et par la musique - bien que l'expression dans des oeuvres comme Ewartung soit moins communication expressive qu'éruption de couches plus 
profondes-, voulait être compris et recherchait la sympathie. On peut sans doute, en faisant abstraction des formules rigoureuses de la métaphysique de l'art, approcher au mieux la fonction actuelle du concept d'oeuvre si l'on se souvient que Friedrich Schleiermacher, dans son projet d'une théorie de l'exégèse textuelle qu'il nomma herméneutique, partit du modèle du dialogue, qui a besoin d'un certain degré d'aide et de soutien pour être véritablement un dialogue de compréhension. Selon le New Criticism, qui à l'insu des acteurs représentait la vraie esthétique ou poétique de la musique sérielle, une des règles fondamentales de l'exégèse textuelle était de ne pas se préoccuper des intentions de l'auteur, de suspecter sa prise en considération d'“intentional fallacy", et de déclarer l'oeuvre elle-même, abstraite du compositeur et de ses vues, seule instance d'une réception adéquate. Mais du fait que la perception esthétique, aussi impartiale se prétende-t-elle, est toujours préconstruite en une forme qui n'est guère vérifiable, on courrait le risque que le texte, qui n'est en premier ressort rien d'autre que la lettre morte de l'oeuvre, devienne l'occasion de projections subjectives se prenant à tort pour l'esprit objectif de l'oeuvre. Et de nombreux compositeurs expliquèrent même qu'ils ne livraient rien de plus qu'un faisceau de stimulus acoustiques, dont la structuration était l'affaire exclusive de l'auditeur.

A l'inverse, le modèle apparemment périmé du dialogue de Schleiermacher permet de préserver la possibilité que les moments que le récepteur apporte avec lui et ceux qui lui viennent depuis l'oeuvre entrent en interaction et s'accordent mutuellement. Toujours est-il qu'entre l'illusion qu'un texte parle de lui-même - on devrait donc uniquement adopter ce qu'il dit, en s'effaçant soi-même - et le coup de force qui veut que le seul sujet de l'auditeur aie en réalité la parole lors de la réception, il existe une médiation qui ne paraît pas impensable.

Si l'on part du fait qu'un concept d'oeuvre non-aliéné, qui autorise la possibilité d'une réception d'après le modèle du dialogue, et un concept de communication concret, qui évite les guet-apens de l'abstraction et admet en son principe la nécessité d'une médiation interactive au moyen d'un objet commun, ne s'excluent pas, on peut alors circonscrire la conception qui est à la base de la relation actuelle, dégagée de toute crispation sur des positions extrêmes, entre l'idée restituée de l'oeuvre et l'idée malgré tout conservée de la communication.

\section{NOTES}

1. Tönende Sinzusammenhang: catégorie de base de l'esthétique adornienne, selon laquelle d'une part le contexte en tant que tel garantit la signification et d'autre part la signification expressive gestuelle associée à un motif musical s'inscrit dans un contexte qui la définit et la modifie - de manière analogue à la façon dont un mot s'insère dans une phrase. [ndt] 


\section{NOTES DE FIN}

*. Ce texte du grand musicologue allemand Carl Dahlhaus, récemment décédé, a paru pour la première fois sous le titre "Abkehr vom Materialdenken ?" in Darmstâdter Beitrâge zur Neuen Musik Bd. XIX (= 31. internationale Ferienkurse für Neue Musik 1980) Schott, Mainz 1982. La présente traduction est dediee à sa mémoire. 


\section{Événements, Instruments, Monuments}

Francine Markovits

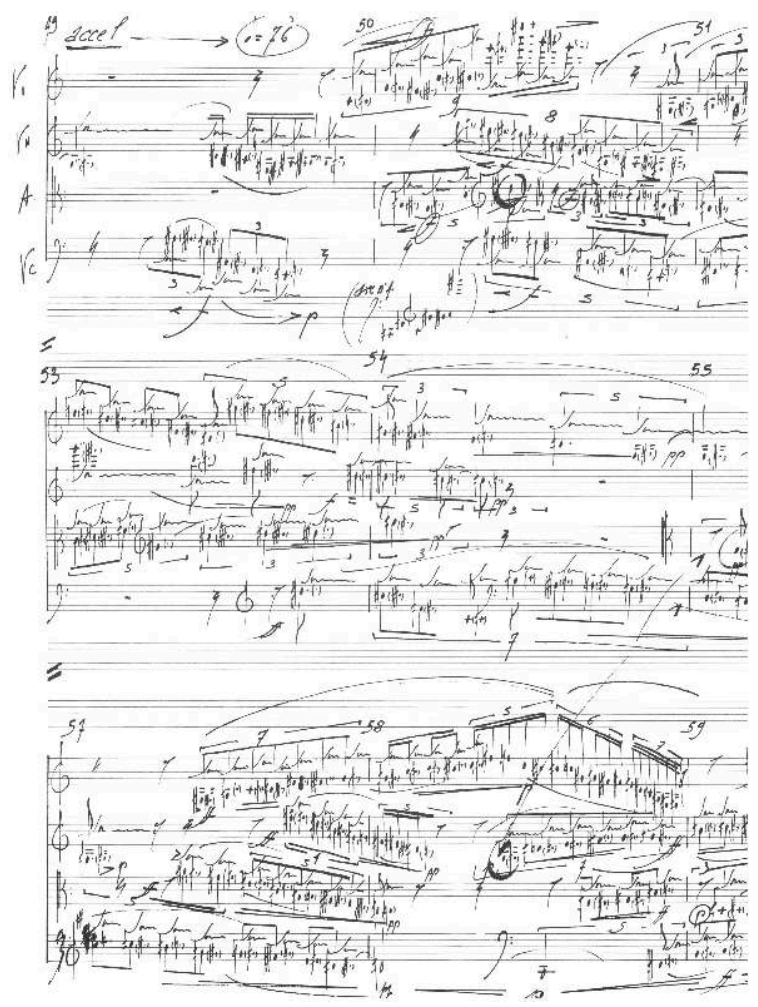




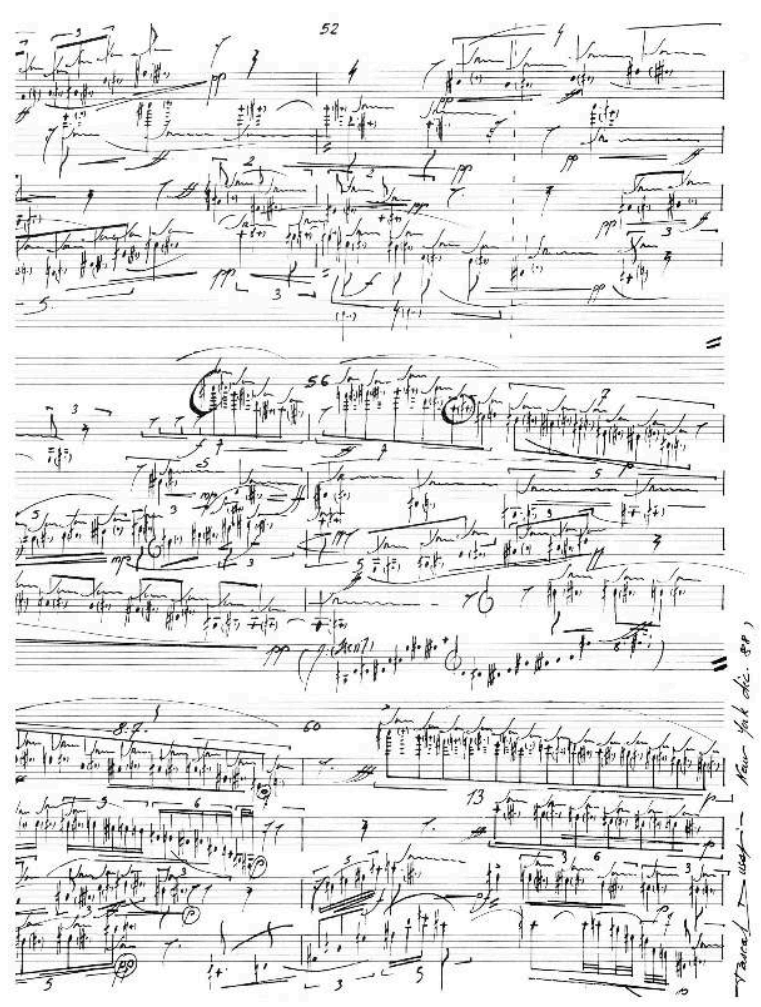

$1 \quad$ Pourquoi quelque chose plutôt que rien?

2 Pourquoi vouloir que quelque chose naisse de rien?

3 Quel mythe est ainsi suscité d'une puissance absolue, paradoxe d'une volonté qui invente l'être? On a adoré ce mystère, on a réduit le mystère à un mythe, on a démasqué les politiques dans les théologiens, puis le mythe de la création s'est réfugié dans l'esthétique.

4 Il faudrait analyser le mythe du créateur comme l'effet d'une demande d'admiration, d'un désir d'impensable. De là naît un culte, celui de la rencontre, où le sujet humain cherche ce qui est radicalement autre: délectation de l'inconnu, voire de l'incommensurable, l'admiration est une passion de l'âme que l'affrontement à l'inouï satisfait en la rendant muette. Autre effet d'admiration, l'horreur devant l'invisible ou le jamais vu, le deinos grec. Ou encore la familiarité paradoxale du dessaisissement, l'inquiétante étrangeté, l'Unheimlichkeit où nous nous rencontrons comme autres.

5 La création serait donc l'effet d'une demande. Il faut déchiffrer l'admiration : vouloir ne pas comprendre, un désir d'inouï qui est un désir de mutisme. Le sujet humain veut s'abolir dans un rêve d'origine. Les vieux codes de la création du monde seraient des histoires héroïques. Les sujets humains s'y éprouvent comme des forces, comme le jeu des éléments. Les hommes s'y pensent ou s'y impensent comme nature.

6 Ce qu'on appelle liberté de la création serait donc la demande d'un retour à un jeu d'éléments, au point où l'infini des possibles ne se structure pas même en combinatoire. Ainsi la création serait-elle transposition d'une histoire, condensation de ses moments dans le chiffre d'une origine. Lorsque nous croyons déchiffrer dans l'origine la naissance des paradigmes, nous composons des monuments. 
7 ... La nature présuppose elle-même la matière, elle ne fait que les formes. Si donc Dieu tirait les choses d'un objet préexistant il ne produirait point cet objet de rien. Or nous avons prouvé que les êtres ne contiennent rien qui ne vienne de Dieu, cause universelle de tout l'être: donc Dieu fait passer les choses du néant à l'être.

8 Saint Thomas, Somme théologique, Traité de la création, Question XLV (traduction F. Lachat), 1863.

9 L'admiration est une subite surprise de l'âme qui fait qu'elle se porte à considérer avec attention les objets qui lui semblent rares et extraordinaires. Ainsi elle est causée premièrement par l'impression qu'on a dans le cerveau, qui représente l'objet comme rare et par conséquent digne d'être fort considéré; puis ensuite par le mouvement des esprits qui sont disposés par cette impression à tendre avec grande force vers l'endroit du cerveau où elle est pour l'y fortifier et conserver; comme aussi ils sont disposés par elle à passer de là dans les muscles qui servent à retenir les organes des sens, en la même situation qu'ils sont, afin qu'elle soit encore entretenue par eux, si c'est par eux qu'elle a été formée.

Descartes, Les Passions de l'âme, article 70, 1649.

11 ... Si la théorie psychanalytique a raison d'affirmer que tout affect d'une émotion, de quelque nature qu'il soit, est transformé en angoisse par le refoulement, il faut que parmi les cas d'angoisse se rencontre un groupe dans lequel on puisse démontrer que l'angoissant est quelque chose de refoulé qui se montre à nouveau. Cette sorte d'angoisse serait justement l'inquiétante étrangeté, l'unheimliche", et il devient indifférent bien que celle-ci ait été à l'origine par ellemême de l'angoisse ou bien qu'elle provienne d'un autre affect. En second lieu, si telle est vraiment la nature intime de l' "unheimliche", nous comprendrons que le langage courant fasse insensiblement passer le "heimliche" à son contraire, l'unheimliche", car cet "unheimliche" n'est en réalité rien de nouveau, d'étranger, mais bien plutôt quelque chose de familier, depuis toujours, à la vie psychique, et que le processus du refoulement seul a rendu autre.

Sigmund Freud, Essais de psychanalyse appliquée, traduction Marty et Marie Bonaparte, Paris, Payot, p. 194.

13 ... La vraie clef de la mythologie est dans l'histoire réelle de tous ces premiers peuples, de leurs opinions et de leurs souverains; dans les fausses traductions d'une quantité d'expressions simples, dont le sens n'était plus entendu de ceux qui continuaient de s'en servir; dans les homonymies qui ont fait autant d'êtres ou de personnes différentes d'un même objet désigné par différentes épithètes. Ils ont vu que la mythologie n'était autre chose que l'histoire ou le récit des actions des morts, comme son nom même l'indique...

14 De Brosses, Le culte des dieux fétiches, 1760 (réédition Fayard, coll. Corpus, 1989).

15 L'Auteur de la nature composa l'univers du concours des atomes; le chaos se développa, et cédant au monde l'espace, leur donna l'impulsion attractive, organisa la voûte azurée, creusa la profondeur des mers; aujourd'hui le concours des liquides trace un nouveau centre, et provoque l'industrie des habitants du globe. La fable nous dit qu'une goutte de lait échappée du sein de Junon produisit la voie lactée; ici c'est une goutte d'eau suspendue en l'air qui acquiert en tombant une valeur progressive, et fonde la ville dont vous voyez le plan de masse, tracé sur la carte générale du pays.

Claude-Nicolas Ledoux, Carte générale des environs de la ville de Chaux, in L'œuvre et les rêves de Ledoux, par Y. Christ, I. Schein, J. Ohayon, Paris, éd. du Chêne, 1971.. 


\section{Rien de nouveau, dit le soleil} Les modèles cycliques sont une réflexion sur le diamètre du cercle, sur la symétrie et les contraires. Des révolutions des planètes aux révolutions des empires, la théorie de la périodicité implique le passage par des positions contraires.

encer ou recommencer est donc définir, pour un système logique donné, des frontières et des positions, une incompatibilité aussi avec d'autres positions. Par un effet de retournement, ce qui est exclu devient règle, le centre change de place, l'observateur devient le mobile. La philosophie du nouveau est-elle une philosophie du pourquoi pas? Inventer de nouvelles lois est susciter la valeur paradigmatique de ce qu'elles excluaient. Inventer, est-ce donc inverser?

Mais faut-il faire confiance aux abstractions de la symétrie ? La philosophie du nouveau n'est-elle pas la philosophie de l'infiniment petit qui dénature le tout ? Contre les effets de totalisation de la périodicité, une philosophie du détail: ne changez qu'un point et observez la transmission continue de cette altération dans le tout. Le détail est l'abrégé d'une succession infinie de causes et d'effets. Ce n'est pas qu'on change l'orientation du regard mais c'est qu'au lieu de regarder un spectacle, on isole les parties. Car l'articulation de chaque partie est abrégée du tout.

La période mise à part, l'autre modèle de l'invention serait dans l'analyse et la subversion du détail avec cette hypothèse que le détail est signe du tout et que les lois de l'ensemble s'y réfléchissent. Mais là encore, deux logiques s'affronteraient, celle de la miniature et celle du monogramme. Deux formes d'abrégé, la miniature change l'échelle, le monogramme change l'ordre.

Pourquoi n'assurerais-je pas des mondes, ce que je crois des animaux? Combien de mondes estropiés, manques, se sont dissipés, se reforment et se dissipent peut-être à chaque instant dans des espaces éloignés, où je ne touche point, et où vous ne voyez pas, mais où le mouvement continue et continuera de combiner des amas de matière, jusqu'à ce qu'ils aient obtenu quelque arrangement dans lequel ils puissent persévérer? o philosophes! Transportez-vous donc avec moi sur les confins de cet univers, au-delà du pont où je touche, et où vous voyez des êtres organisés; promenez-vous sur ce nouvel océan, et cherchez à travers ses agitations irrégulières quelques vestiges de cet être intelligent dont vous admirez ici la sagesse!

"Mais à quoi bon vous tirer de votre élément? Qu'est-ce que ce monde, monsieur Holmes? Un composé sujet à des révolutions, qui toutes indiquent une tendance continuelle à la destruction; une succession rapide d'êtres qui s'entre-suivent, se poussent et disparaissent; une symétrie passagère; un ordre momentané. Je vous reprochais tout à l'heure d'estimer la perfection des choses par votre capacité; et je pourrais vous accuser ici d'en mesurer la durée sur celle de vos jours. Vous jugez de l'existence successive du monde, comme la mouche éphémère de la vôtre. Le monde est éternel pour vous, comme vous êtes éternel pour l'être qui ne vit qu'un instant. Encore l'insecte est-il plus raisonnable que vous. Quelle suite prodigieuse de générations éphémères atteste votre éternité! Quelle tradition immense! Cependant nous passerons tous, sans qu'on 
puisse assigner ni l'étendue réelle que nous occupions, ni le temps précis que nous aurons duré. Le temps, la matière et l'espace ne sont peut-être qu'un point."

\section{Les abeilles et le blues} d'une œuvre qui fasse paradigme. Imiter s'inscrit dans une continuité : l'auteur qui imite peut énoncer tous les moments de la démarche qu'il accomplit. Par opposition à la continuité de ces médiations, créer suppose une discontinuité et une rupture; la transposition du concept théologique dans le concept esthétique promeut une subjectivité souveraine. A la différence cependant de la subjectivité divine, la subjectivité de l'artiste est aveugle sur la finalité de ses opérations, elle produit une œuvre originale sans pouvoir donner la règle de sa production. Cette cécité, cette finalité inconsciente d'elle-même, a pu être interprétée comme l'inspiration d'un dieu. L'artiste est l'instrument du dieu qui l'anime comme l'instrumentiste donne à son instrument le mouvement et la vie. L'artiste est transporté hors de soi, un dieu l'habite à sa place et lui souffle sa parole. Comme le peuple et les siècles s'approprieront sa parole et son œuvre.

30 Cette finalité aveugle a aussi pu être interprétée comme la nature à l'œuvre dans le génie. Les génies seraient les favoris de la nature car leurs opérations sont l'effet d'un don plutôt que d'un travail. Mais ils sont en même temps le chiffre des producteurs de la nature puisqu'ils sont, dans leur individu, la présentation de la finalité naturelle. La production de l'œuvre d'art et la production de la nature ne sont pas l'effet d'une intentionnalité consciente.

31 L'analogie des opérations de la nature et du génie apparaît donc comme la critique d'une causalité de la conscience de soi. Le chiffre de la nature et du génie est la 
condensation des opérations d'un travail. Le travail apparaît comme la subversion de l'idée de création. disais tout à l'heure, mais une puissance divine qui te met en branle, comme dans le cas de la pierre qui a été appelée "magnétique" par Euripide et qu'on appelle le plus souvent pierre d'Héraclée. Cette pierre en effet ne se borne pas à attirer simplement les anneaux quand ils sont en fer, mais encore elle fait passer dans ces anneaux une puissance qui les rend capables de produire ce même effet que produit la pierre et d'attirer d'autres anneaux; si bien que parfois $i l$ se forme une file, tout à fait longue, d'anneaux suspendus les uns aux autres, alors que c'est de la pierre en question que dépend la puissance qui réside en tous ceux-ci. Or c'est ainsi, également, que la Muse, par elle-même, fait qu'en certains hommes est la Divinité, et que, par l'intermédiaire de ces êtres en qui réside un Dieu, est suspendue à elle une file d'autres gens qu'habita alors la Divinité! Ce n'est pas, sache-le, par un effet de l'art, mais bien parce qu'un Dieu est en eux et qu'il les possède, que tous les poètes épiques, les bons s'entend, composent tous ces beaux poèmes, et pareillement pour les auteurs de chants lyriques, pour les bons. De même que ceux qui sont en proie au délire des Corybantes ne se livrent pas à leurs danses quand ils ont leurs esprits, de même aussi les auteurs de chants lyriques n'ont pas leurs esprits quand ils composent ces chants magnifiques; tout au contraire, aussi souvent qu'ils se sont embarqués dans l'harmonie et dans le rythme, alors les saisit le transport bachique, et, possédés, ils ressemblent aux Bacchantes qui puisent aux fleuves le miel et le lait quand elles sont en état de possession, mais non pas quand elles ont leurs esprits. Et ce que disent ces lyriques, leur âme le réalise à la lettre; voilà bien en effet ce qu'ils nous disent à nous, ces poètes, que, puisant à des sources d'où coule le miel, butinant sur certains jardins et bocages des Muses, ils sont pareils aux abeilles quand ils nous apportent leurs vers, et que, comme elles, ils volent eux aussi. Véridique langage! Le poète en effet est chose légère, chose ailée, chose sainte, et il n'est pas encore capable de créer jusqu'à ce qu'il soit devenu l'homme qu'habite un dieu, qu'il ait perdu la tête, tant que cela au contraire sera sa possession, aucun être humain ne sera capable, ni de créer, ni de vaticiner.

Platon, lon, traduction L. Robin, Paris, La Pléiade.

On voit que le génie: 1. est un talent, qui consiste à produire ce dont on ne saurait donner aucune règle déterminée; il ne s'agit pas d'une aptitude à ce qui peut être appris d'après une règle quelconque; il s'ensuit que l'originalité doit être sa première propriété; 2 . que l'absurde aussi pouvant être original, ses produits doivent en même temps être des modèles, c'est-à-dire exemplaires et par conséquent, que sans avoir été eux-mêmes engendrés par l'imitation, ils doivent toutefois servir aux autres de mesure ou de règle du jugement; 3. qu'il ne peut décrire lui-même ou exposer scientifiquement comment il réalise son produit, et qu'au contraire c'est en tant que nature qu'il donne la règle; c'est pourquoi le créateur d'un produit qu'il doit à son génie, ne sait pas lui-même comment se trouvent en lui les idées qui s'y rapportent et il n'est en son pouvoir ni de concevoir à volonté ou suivant un plan telles idées, ni de les communiquer aux autres dans les préceptes, qui les mettraient à même de réaliser des produits semblables (c'est pourquoi aussi le mot génie est vraisemblablement dérivé de genius, l'esprit particulier donné à un homme à sa naissance pour le protéger et le diriger, et qui est la source de l'inspiration dont procèdent ces idées originales); 4. que la nature par <durch> le génie ne prescrit pas de règle à la science, mais à l'art; et que cela n'est le cas que s'il s'agit des beaux-arts. 


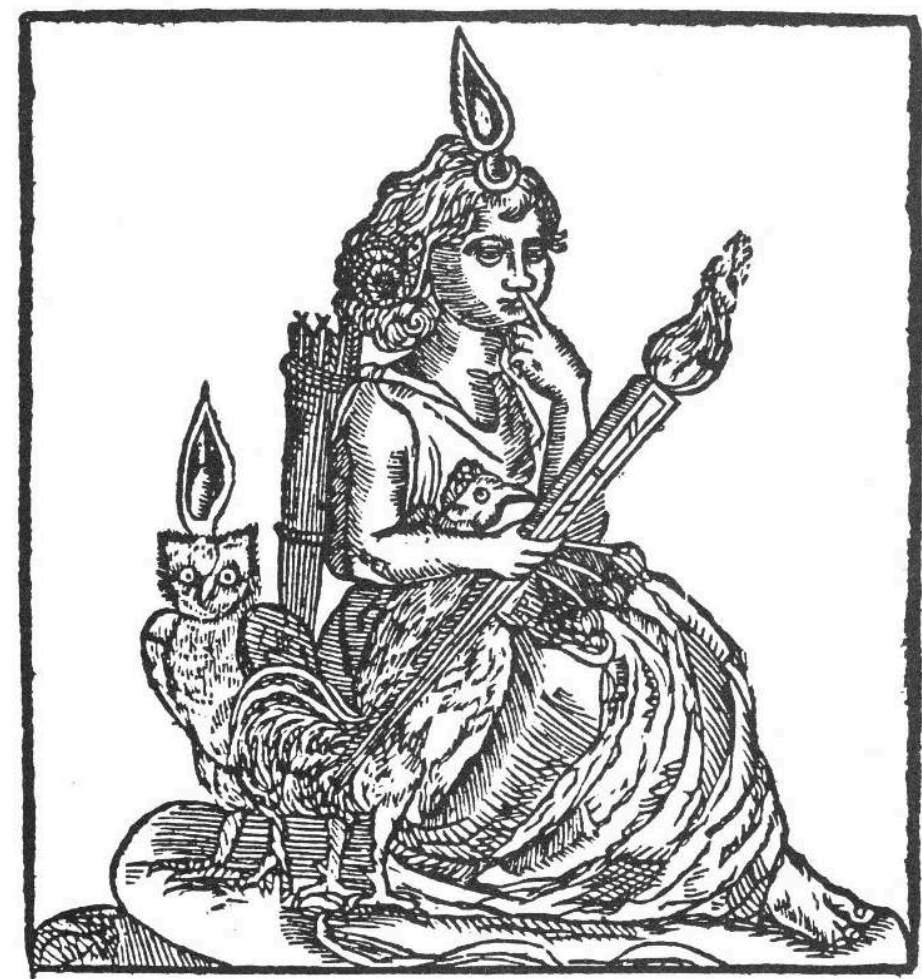

Athanasius Kircher. Harpocrate, dieu gréco-égyptien du silence (Oedipus Aegytiacus, III, 1654). Tiré de Athanasius Kircher, de Joscelyn Godwin, édition originale 1979, Thames and Hudson. Edition française Jean-Jacques Pauvert

Le génie est frappé de tout; et dès qu'il n'est point livré à ses pensées et subjugué par l'enthousiasme, il étudie, pour ainsi dire, sans s'en apercevoir; il est forcé, par les impressions que les objets font sur lui, à s'enrichir sans cesse de connaissances qui ne lui ont rien coûté; il jette sur la nature des coups d'oeil généraux et perce ses abîmes. Il recueille dans son sein des germes qui $y$ entrent imperceptiblement, et qui produisent dans le temps des effets si surprenants, qu'il est lui-même tenté de se croire inspiré : il a pourtant le goût de l'observation; mais il observe rapidement un grand espace, une multitude d'êtres.

Le mouvement, qui est son état naturel, est quelquefois si doux qu'à peine il l'aperçoit: mais le plus souvent ce mouvement excite des tempêtes, et le génie est plutôt emporté par un torrent d'idées, qu'il ne suit librement de tranquilles réflexions. Dans l'homme que l'imagination domine, les idées se lient par les circonstances et par le sentiment; il ne voit souvent des idées abstraites que dans leur rapport avec les idées sensibles. Il donne aux abstractions une existence indépendante de l'esprit qui les a faites; il réalise des fantômes; son enthousiasme augmente au spectacle de ses créations, c'est-à-dire, de ses nouvelles combinaisons, seules créations de l'homme. Emporté par la foule de ses pensées, livré à la facilité de les combiner, forcé de produire, il trouve mille preuves spécieuses, et ne peut s'assurer d'une seule; il construit des édifices hardis que la raison n'oserait habiter, et qui lui plaisent par leurs proportions et non par leur solidité; il admire ses systèmes comme il admirerait le plan d'un poème; et il les adopte comme beaux, en croyant les aimer comme vrais.

Diderot, article “Génie”, de l’Encyclopédie. 


\section{La bibliothèque des instruments} passait pas par une différence de statut entre l'artisan et l'artiste, entre l'ingénieur et le génie. Arts et techniques étaient liés au monde du travail, l'école d'un maître était un atelier. Inventer n'était pas synonyme de créer. Les ingénieurs de la Renaissance inventent des machines mais font aussi œuvre de peintres, de sculpteurs, d'architectes. La définition de leur génie est objective plutôt que subjective : gloire de leurs ouvrages, prestige de leurs cités. De l'Antiquité au Moyen Age, les traités de mécanique sont recopiés et commentés et l'invention repose sur la continuité de la transmission. Pour comprendre l'invention, il ne s'agit pas d'identifier et d'isoler une cause mais de marquer des concomitances: déplacement des livres et des techniques, problèmes posés par une situation déterminée, militaire ou civile, changement des institutions et des pouvoirs. Il faut penser des rencontres. Le génie ne crée pas, il n'est pas cause de l'invention, il en fait partie.

Inventer, c'est donc transposer des pratiques, traduire des techniques, changer le domaine d'application d'une hypothèse. Entre les essais scientifiques et les expériences esthétiques, un savoir circule. L'unité des arts et des techniques suppose à la fois une encyclopédie, une systématisation des connaissances et une unité entre la théorie et la pratique. Le modèle expériemental fait l'unité. Leibniz définit l'art d'inventer comme Bibliothèque universelle et Théâtre de la pratique des hommes. Toute pratique est un savoir condensé, toute théorie est analyse d'une expérience. Inventer, en ce sens, c'est déchiffrer, énoncer ce qui était latent, démonter les dispositifs du sens, déployer la polysémie de ces dispositifs, expérimenter d'autres constructions.

Dans ce contexte, la création s'oppose au travail. Pour l'historien comme pour l'ingénieur, c'est le détail des médiations qui instruit. L'art d'inventer permettrait de récuser les modèles de l'originalité et du génie et de chercher ce qui, de l'incommensurable et de la création, s'était déplacé dans les idées esthétiques.

Mais le concept de création ne se laisse pas seulement critiquer par le travail. La finalité de la création est également exclue par le jeu. Contre les providentialismes, contre le modèle finalisé de la conscience de soi, Nietzsche suscite le mythe héraclitéen de l'enfant et du jeu. L'absence de finalité nous fait penser la causalité du monde en termes de distribution. Loin d'individualiser les éléments, de leur assigner un rôle, le hasard les disperse : toute unité est effet de rencontre.

Après avoir dit que la création et le génie ne sont pas la clé de l'œuvre d'art, peut-on assigner ce rôle au jeu et au hasard? La théologie ne reprendrait-elle pas subrepticement ses droits au moment où s'instituerait un autre culte de la singularité, celui de la rencontre et de l'événement? Peut-on transformer en finalité ce qui était critique de la finalité ? Car le paradoxe de l'art aujourd'hui, c'est qu'en intégrant les nouvelles matières, les nouvelles techniques, les nouvelles machines, les phénomènes de marché, les collections et les événements, la réflexion sur les phénomènes de la vie artistique, de la vie esthétique, se situe à la croisée de deux logiques: l'une pense les médiations historiques de toute production et dénie l'efficace du génie et la création; l'autre, en promouvant la singularité des expériences contre la monumentalisation des œuvres d'art, ranime, avec le culte de l'instant, le privilège de l'incommensurable. 

différentes professions, je suis persuadé qu'ils passent de beaucoup tant à l'égard de la multitude que de l'importance, tout ce qui se trouve marqué dans les livres, et que la meilleure partie de nostre trésor n'est pas encor enregistrée. Il y en a même tousjours qui sont particulières à certaines personnes et se perdent avec elles. Il n'y a point d'art mécanique si petit et si méprisable, qui ne puisse fournir quelques observations ou considérations remarquables, et toutes les professions ou vocations ont certaines adresses ingénieuses dont il n'est pas aisé de s'aviser et qui neantmoins peuvent servir à des conséquences bien plus relevées. On peut adjouter que la matière importante des manufactures et du commerce ne sçauroit estre bien réglée que par une exacte description de ce qui appartient à toute sorte d'arts, et que les affaires de milice et finances et de marine dépendent beaucoup des mathématiques et de la physique particulière. Et c'est là le principal défaut de beaucoup de sçavants qu'ils ne s'amusent qu'à des discours vagues et rebattus, pendant qu'il y a un si beau champ à exercer leur esprit dans des objets solides et réels à l'avantage du public. Les chasseurs, les pêcheurs, les mariniers, les marchands, les voyageurs et même les jeux tant d'adresse que de hasard fournissent de quoy augmenter considérablement les sciences utiles. Il y a jusque dans les exercices des enfants ce qui pourroit arrester le plus grand Mathématicien; apparemment nous devons l'aiguille aimantée à leur amusements, car qui se seroit avisé d'aller regarder, comment elle se tourne, et il est constant que nous leur devons l'arquebuse à vent, qu'ils practiquoient avec un simple tuyau de plume qu'ils bouchoient par les deux bouts en perçant tantost avec l'un bout et tantost avec l'autre la tranche d'une pomme, forçant par après un bouchon d'approcher de l'autre et de la chasser à force de l'air pressé entre deux, long temps avant qu'un habile ouvrier Normand s'avisa de les imiter en grand. Enfin sans négliger aucune observation extraordinaire, il nous faut un véritable Théâtre de la vie humaine tiré de la practique des hommes bien différent de celuy que quelques sçavants hommes nous ont laissé, dans lequel tout grand qu'il est. il n'y a guères que ce qui peut servir à des harangues et à des sermons. Pour concevoir ce qu'il nous faudroit choisir pour ces descriptions réelles et propres à la practique, on n'a qu'à se figurer de combien de lumières on auroit besoin pour se pouvoir faire à soy même dans une isle déserte, ou faire faire par des peuples barbares, si on s'y trouvoit transporté par un coup du vent, tout ce qui nous peut fournir d'utile et de commode l'abondance d'une grande ville toute pleine des meilleurs ouvriers et des plus habiles gens de toutes sortes de conditions; ou bien il faut s'imaginer qu'un art fut perdu et qu'il le faudroit retrouver, à quoy souvent toutes nos Bibliothèques ne pourroient suppléer, car bien que je ne disconvienne pas qu'il y a en revanche beaucoup de belles choses dans les livres, que les gens de profession ignorent encor eux mêmes, et dont ils pourroient profiter, il est constant neantmoins que les plus considérables observations et tours d'adresse en toute sorte de mestiers et de professions sont encor non-écrits. Ce qu'on trouve par expérience lorsqu'en passant de la théorie à la pratique, on veut exécuter quelque chose. Ce n'est pas que cette pratique ne se puisse écrire aussi, puisqu'elle n'est dans le fonds qu'une autre théorie, plus composée et plus particulière que la commune; mais les ouvriers pour la pluspart outre qu'ils ne sont pas d'humeur à enseigner autres que leur apprentifs, ne sont pas des gens à s'expliquer intelligiblement par écrit, et nos auteurs sautent par dessus ces particularités lesquelles bien qu'essentielles ne passent chez eux que pour des minuties, dont ils ne daignent pas de s'informer, outre la peine qu'il y a de les bien décrire.

$$
\text { VII, p. 181-182. }
$$

Subversion de l'incommensurable : il reste à penser, dans la création esthétique, moins des effets de rupture qu'un effet de surprise et de dessaisissement. Une universelle 
communicabilité du sensible et de l'expérience du beau. Kant y repère une unanimité de l'humanité, et pour cela, la musique est exemplaire d'être la langue universelle des sensations. Marx s'interroge sur un phénomène qui défie la formation historique des hommes.

De Mallarmé au futurisme et au dadaïsme, les puissances du verbe ont exténué la littérature et la critique. Après l'alchimie et les combinatoires, il semble que nous retrouvions aujourd'hui la problématisation qui fut celle du XVIII siècle et que les interprètes de l'Aufklärung ont éludée : les discours sont des pratiques; les pratiques sont des savoirs muets. On avait alors déjà substitué à la recherche de l'origine la question de la communicabilité et dans cette question on avait déjà contesté la nécessité d'une commune mesure universelle. Autant que la recherche de l'originel, la recherche de l'original fut récusée comme l'effet d'une demande. La réduction de l'illusion $\mathrm{du}$ radicalement autre, de l'absolument nouveau s'opère dans une problématique de la traduction. Mais s'il s'agissait simplement de réduire la demande de l'archaïque ou la demande de l'universel pour restituer la vérité, on serait encore pris au piège de la finalité de la conscience de soi.

Inventer, c'est changer les voies d'accès. Traduire.

La musique puise le grand art de remuer les passions [dans] l'instinct donné à tous les hommes, à tous les animaux mêmes, d'exprimer par certains sons, ou même, si vous voulez, par certains cris, les diverses passions qui les affectent.

L'homme, avant l'art de la parole, n'avait point d'autre langage: celui-ci est le même pour tous les peuples, dans tous les climats: c'est pourquoi la musique est la vraie langue universelle: et elle n'est autre chose que l'art d'exprimer ces cris avec mélodie ou de les transformer en sons harmonieux : et comme il semble que les éléments mêmes entrent pour quelque chose dans cette espèce de concert d'expressions des passions: que la tempête et le tonnerre par exemple, impriment un effroi qui les a fait regarder comme les signes et les instruments de la colère des Dieux, etc, La Musique, en imitant les scènes du spectacle de la nature, excite en nous les passions qui y sont attachées.

51 Le Cat, Traité des sensations et des passions en général et des sens en particulier, Paris, 1768.

52 Ne cherche point, jeune artiste, ce que c'est que le génie. En as-tu, tu le sens en toi-même. N'en as-tu pas, tu ne le connoîtras jamais. Le génie du musicien soumet l'univers entier à son art; il peint tous les tableaux par des sons; il fait parler le silence même; il rend les idées par des sentiments, les sentiments par des accents, et les passions qu'il exprime, il les excite au fond des coeurs; la volupté, par lui, prend de nouveaux charmes; la douleur qu'il fait gémir arrache des cris; il brûle sans cesse, et ne se consume jamais : il exprime avec chaleur les frimas et les glaces; même en peignant les horreurs de la mort, il porte dans l'ame ce sentiment de vie qui ne l'abandonne point, et qu'il communique aux coeurs faits pour le sentir; mais hélas ! il ne sait rien dire à ceux où son germe n'est pas, et ses prodiges sont peu sensibles à qui ne peut les imiter. Veux-tu donc savoir si quelque étincelle de ce feu dévorant t'anime; cours, vole à Naples écouter les chefs-d'œuvre de Leo, de Durante, de fornelli, de Pergolèse. Si tes yeux s'emplissent de larmes, si tu sens ton coeur palpiter, si des tressaillements t'agitent, si l'oppression te suffoque dans tes transports, prends le Métastase, et travaille; son génie échauffera le tien, tu créeras à son 
exemple; c'est là ce que fait le génie, et d'autres yeux te rendront bientôt les pleurs que les maîtres t'ont fait verser. Mais si les charmes de ce grand art te laissent tranquille, si tu n'as ni délire ni ravissement, si tu ne trouves que beau ce qui transporte, oses-tu demander ce qu'est le génie ? Homme vulgaire, ne profane point ce nom sublime. Que t'importerait de la connoître? tu ne saurais le sentir :fais de la musique françoise. natures à la fois? Ce que selon votre représentation vous appelez limitation, je l'appelle selon la mienne - et je l'ai justifiée - extension. A l'inverse de votre conception, je dirais donc plutôt: l'agréable, le bien, la perfection, l'homme les prend seulement au sérieux, mais avec la beauté il joue.

\section{Le clavecin des couleurs}

En jouant sur les analogies de l'audible et du visible, le Père Castel, Jésuite, inventa au XVIII siècle un clavecin oculaire. Il s'agissait de peindre la musique avec ses propres couleurs, de manière qu'un sourd puisse jouir et juger de la beauté d'une musique et un aveugle de la beauté des couleurs. Mais aussitôt, on pensa aussi à un catalogue des parfums, à une grammaire du toucher. Et la grande question était de savoir si ces traductions mécaniques supposaient ou non une équivalence de toutes ces gammes.

clavecin des couleurs jouait donc des harmonies chromatiques et musicales: mais cette simultanéité fait penser. Diderot conduit un sourd-muet de naissance devant le clavecin oculaire du Père Castel. Celui-ci pensait qu'on pouvait par un simple jeu démontrer toute la suite des Eléments d'Euclide : la géométrie est une machine. Pascal et Leibniz l'auraient avoué. Mais le sourd-muet vit dans le clavecin oculaire une machine à communiquer les pensées, ce qui est un peu différent de la démonstration, et concerne la communication de l'émotion et l'intelligence du non-dit. Comment ne pas imaginer qu'il y ait du bon sens dans les sons, demande Diderot, puisque la musique et le discours produisent en nous les mêmes signes des passions?

Dans cette machinerie, l'instrumentiste fait partie du dispositif, comme dans l'analyse du discours, le sujet de la parole fait partie des énoncés. La version instrumentiste de la 
rhétorique de l'invention dit que les dispositifs conduisent le génie. Démontez les machines.

61 Créer est inventer, inventer est déjouer l'intention d'une construction, changer les dispositifs du sens. Briser le consensus, déployer une polysémie. Désaccorder, déconcerter. Du consensus à la dissonance.

Pour réduire l'illusion de la création et penser l'invention, deux voies se proposent. L'une est le jeu: restituer l'arbitraire de la combinaison à l'infinie variation des possibles. Tous les calculs sont permis.

L'autre voie est le travail des signes. C'est alors le législateur qui nous donne le modèle du génie. D'un système de droit à l'autre, le législateur traduit et interprète. Il démonte les dispositifs qui ont façonné la nature humaine. Si l'on change les temps et les lieux, les mêmes lois ne produisent pas les mêmes effets. Car ce sont les situations et les circonstances de l'histoire qui produisent les systèmes de normes. Montesquieu nous a appris à penser les lois comme des fonctions. C'est ainsi la figure du législateur qui nous donne le paradigme de l'art d'inventer. En empruntant à l'art du législateur, l'esthétique de la création devrait donc repenser le rapport de la sensibilité à l'histoire. Changer la sensibilité pour changer la raison.

Vous connaissez, au moins de réputation, une machine singulière, sur laquelle l'inventeur se proposait d'exécuter des sonates de couleurs. J'imaginai que s'il y avait un être au monde qui dût prendre quelque plaisir à de la musique oculaire, et qui pût en juger sans prévention, c'était un sourd et muet de naissance. Je conduisis donc le mien rue Saint-Jacques, dans la maison où l'on montre l'homme et la machine aux couleurs. Ah! Monsieur, vous ne devinerez jamais l'impression que ces deux êtres firent sur lui, et moins encore les pensées qui lui vinrent.

Vous concevez d'abord qu'il n'était pas possible de lui rien communiquer sur la nature et les propriétés merveilleuses du clavecin; que n'ayant aucune idée du son, celles qu'il prenait de l'instrument oculaire n'étaient assurément pas relatives à la musique, et que la destination de cette machine lui était tout aussi incompréhensible que l'usage que nous faisons des organes de la parole. Que pensait-il donc? et quel était le fondement de l'admiration dans laquelle il tomba, à l'aspect des éventails du Père Castel ? Cherchez, monsieur; devinez ce qu'il conjectura de cette machine ingénieuse, que peu de gens ont vue, dont plusieurs ont parlé, et dont l'invention ferait bien de l'honneur à la plupart de ceux qui en ont parlé avec dédain; ou plutôt, écoutez, le voici : Mon sourd s'imagina que ce génie inventeur était sourd et muet aussi; que son clavecin lui servait à converser avec les autres hommes; que chaque nuance avait sur le clavier la valeur d'une des lettres de l'alphabet ; et qu'à l'aide des touches et de l'agilité des doigts, il combinait ces lettres, en formait des mots, des phrases; enfin, tout un discours en couleurs.

Après cet effort de pénétration, convenez qu'un sourd et muet pouvait être assez content de luimême; mais le mien ne s'en tint pas là ; il crut tout d'un coup qu'il avait saisi ce que c'était que la musique et tous les instruments de musique. Il crut que la musique était une façon particulière de communiquer la pensée, et que les instruments, les vielles, les violons, les trompettes étaient, entre nos mains, d'autres organes de la parole.

Diderot : Lettre sur les sourds et les muets, 1751. 


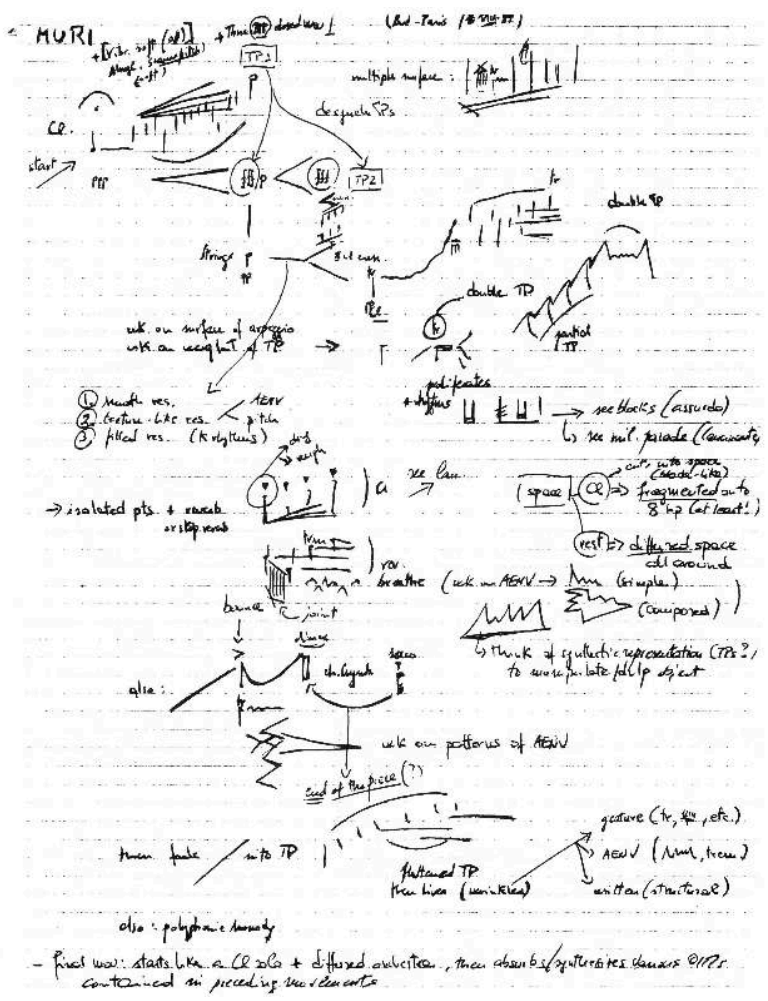

La Fondation Total pour la Musique et le Festival d'Automne à Paris 1989

La commémoration du Bicentenaire de la Révolution Française a fait peu de place aux commandes d'œuvres artistiques nouvelles. La Fondation Total pour la Musique a souhaité honorer l'acte de naissance d'une société renouvelée, par un geste qui souligne que l'avenir de notre culture est toujours, dans sa continuité et ses ruptures, en gestation.

Partageant cette approche avec le Festival d'Automne à Paris, la Fondation Total pour la Musique a décidé de s'associer à lui dans un important programme de commandes et de créations d'œuvres nouvelles, de quelques-uns des plus marquants parmi les compositeurs d'aujourd'hui : Pierre Boulez (Explosante-Fixe), Jean-Claude Eloy (Rosa, Mirra... chants de libération pour deux voix solistes de femmes), Helmut Lachenmann (Quatuor à cordes), Philippe Manoury et Marco Stroppa.

La Fondation donne ainsi une ampleur plus grande, mais aussi plus de visibilité à une politique déjà éprouvée en faveur de la musique contemporaine. Cette politique s'est appuyée sur les commandes et les co-productions de grands concerts de création, de festivals ou de cycles thématiques, sur l'édition de disques et de livres.

Philippe MOROT

Directeur des programmes de la Fondation Total pour la Musique 

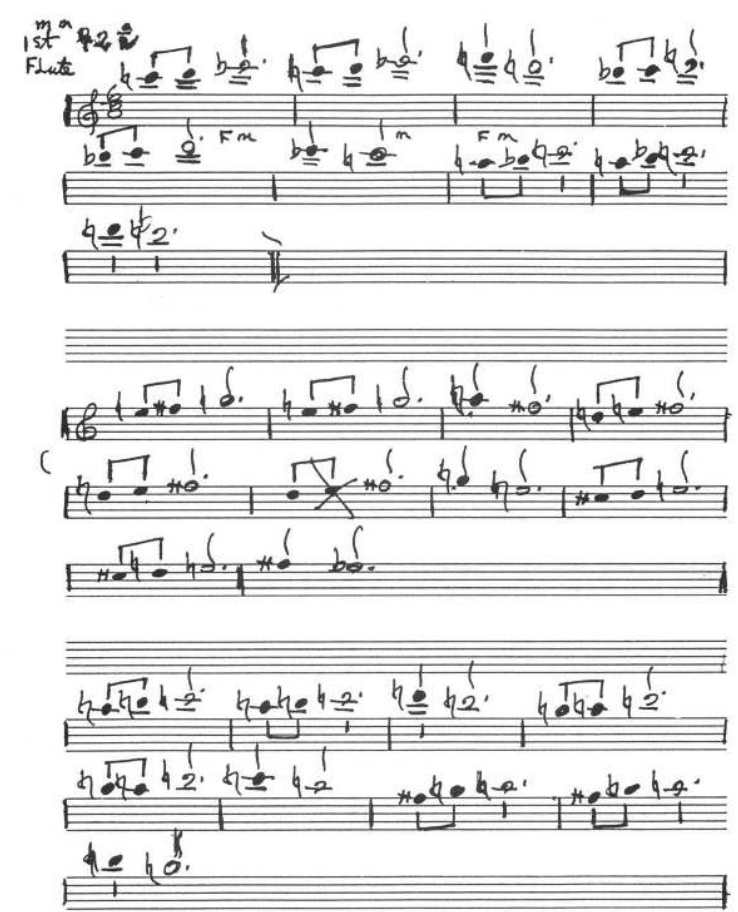

La Caisse des dépôts et consignations et le Festival d'Automne à Paris 1989 La Caisse des dépôts et consignations a choisi de mettre l'accent, dans sa politique de mécénat culturel, sur la création contemporaine.

La musique du $\mathrm{XX}^{\mathrm{e}}$ siècle y prend une place toute particulière, prolongeant l'action

menée en faveur du Théâtre des Champs-Elysées.

Ainsi, depuis trois ans, le Centre Acanthes bénéficie d'un important concours. Ce soutien lui a permis d'organiser, dans le cadre propice de la Chartreuse de Villeneuve lez-Avignon, des cycles de cours et de concerts où, tour à tour, Olivier Messiaen, Pierre Boulez et, cette année, Luigi Nono ont rencontré des stagiaires assidus et un public nombreux.

De même, associée dès l'an passé au Festival d'Automne qui célébrait l'anniversaire de Karlheinz Stockhausen et d'Olivier Messiaen, la Caisse des dépôts a renforcé cette année cette collaboration en parrainant la réalisation d'une dizaine de concerts et la commande passée à Karlheinz Stockhausen, dont la création est prévue pour 1990.

La publication de cet ouvrage est un complément opportun et heureux à cette nouvelle édition du Festival d'Automne. Elle constitue un document important sur l'état de la création musicale contemporaine. La Caisse des dépôts ne pouvait pas ne pas s'y associer.

Pierre LEBAILLIF

Chargé de mission pour le mécénat et l'action culturelle à la Caisse des dépôts et consignations

La Fondation Louis Vuitton pour l'Opéra et la Musique et le Festival d'Automne à Paris 1989

Pour la troisième année consécutive, la Fondation Louis Vuitton pour l'Opéra et la 
Musique tient à apporter son aide aux manifestations musicales du Festival d'Automne à Paris, renforçant ainsi les liens d'amicale confiance tissés avec son président, Michel Guy.

Déjà présente lors de la création française du "Prometeo" de Luigi Nono, au Théâtre de Chaillot en 1987, et lors de la création française de "Montag" de Karlheinz Stockhausen, journée du cycle "Licht", au Théâtre des Champs-Elysées en 1988, la Fondation participe cette année à la commande et à la création au Théâtre du Châtelet, le 18 décembre 1989, de deux œuvres de Luciano Berio et Pierre Boulez.

Cette opération s'inscrit dans le cadre du programme de commandes passées par le Festival d'Automne à une trentaine de compositeurs pour la commémoration du Bicentenaire de la Révolution Française.

Ce projet ne pouvait que séduire la Fondation Louis Vuitton pour l'Opéra et la Musique qui, dans ce même esprit, vient de participer à la commande et à la création, cet été, à l'Opéra de Montpellier et au Festival d'Avignon, de "Roméo et Juliette", opéra de Pascal Dusapin et Olivier Cadiot, ainsi qu'à la "Fête de la Création", organisée au Palais des Papes par le Centre Acanthes.

La Fondation Louis Vuitton réaffirme ainsi sa fidélité à l'objectif principal défini voici quatre ans lors de la réunion constitutive de son Comité Artistique, l'aide prioritaire à la musique de notre temps, au plus près des mécanismes de la création.

Henri RACAMIER

Président de la Fondation Louis Vuitton pour l'Opéra et la Musique 